\title{
Attention shift and remapping across saccades
}

\author{
Dissertation \\ for the award of the degree \\ "Doctor of Philosophy" Ph.D. Division of Mathematics and Natural Sciences \\ of the Georg-August-Universität Göttingen \\ within the doctoral program Basic Program in Biology \\ of the Georg-August University School of Science (GAUSS) \\ Submitted by \\ Tao Yao \\ from Hunan, China
}

Göttingen, November 2016 
Thesis Committee:

Prof. Dr. Stefan Treue (supervisor),

Cognitive Neuroscience Laboratory, German Primate Center

Prof. Dr. Alexander Gail,

Sensorimotor Group, Cognitive Neuroscience Laboratory, German Primate Center

Dr. Suresh Krishna (supervisor),

Cognitive Neuroscience Laboratory, German Primate Center

Members of the examination board:

Referee: Prof. Dr. Stefan Treue,

Cognitive Neuroscience Laboratory, German Primate Center

Co-referee: Prof. Dr. Uwe Mattler,

Georg-Elias-Müller-Institut für Psychologie, University of Göttingen

Other members of the Examination Board:

Prof. Dr. Ralf Heinrich,

Department of Cellular Neurobiology, Johann-Friedrich-Blumenbach-Institute for Zoology and Anthropology, University of Göttingen

Prof. Dr. Tim Gollisch,

Department of Ophthalmology, University Medical Center Göttingen

Prof. Dr. Alexander Gail,

Sensorimotor Group, Cognitive Neuroscience Laboratory, German Primate Center

Prof. Dr. Andreas Stumpner,

Department of Cellular Neurobiology, Johann-Friedrich-Blumenbach-Institute for Zoology and Anthropology, University of Göttingen

Date of the oral examination: 19.12.2016 
Herewith I declare that I have written this thesis independently and with no other aids and sources than quoted.

Göttingen, 01.11.2016

(Tao Yao) 



\section{Contents}

\section{CHAPTER}

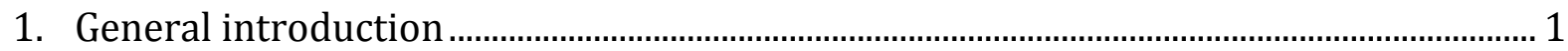

2. Attentional remapping in macaque area MT is well-synchronized to saccades ..........41

3. Visual attention is available at a task-relevant location rapidly after a saccade .........93

4. An attention-sensitive memory trace in macaque MT following saccadic eye movements

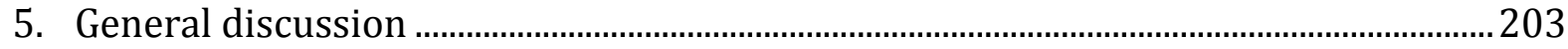

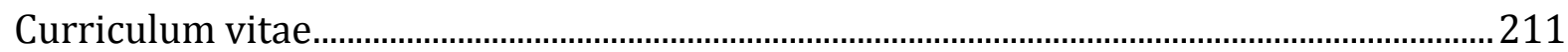



Chapter 1

General introduction 


\section{Content}

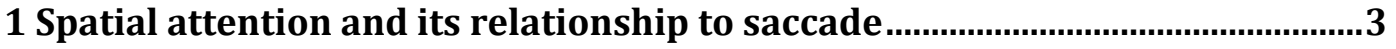

1.1 Attention and its functions ...........................................................................

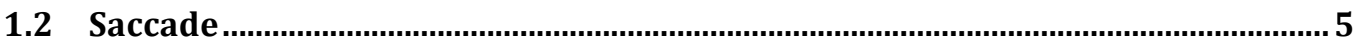

1.2.1 Saccadic suppression .....................................................................................................

1.3 Relationship between spatial attention and saccades...................................... 8

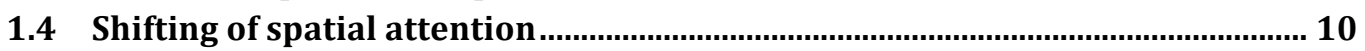

1.4.1 Attention shift with fixation.........................................................................................

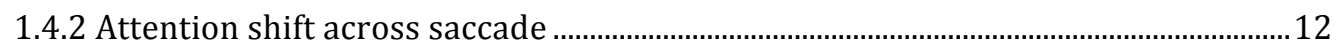

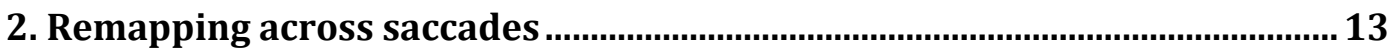

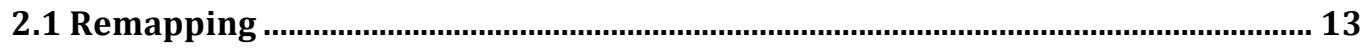

2.1.1 Physiological studies................................................................................................... 14

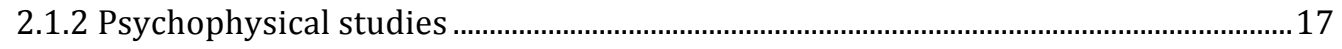

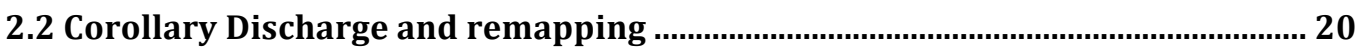

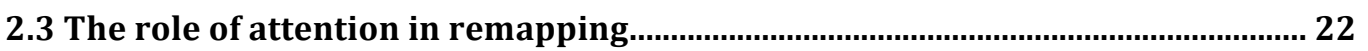

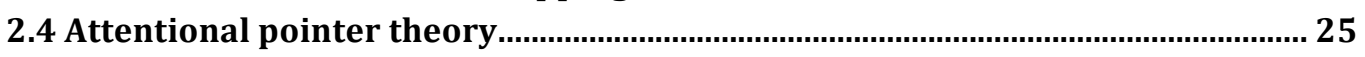

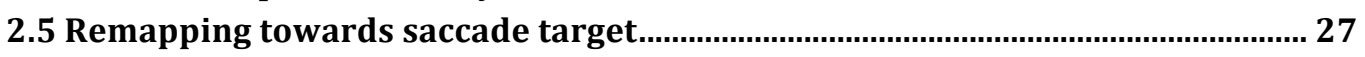

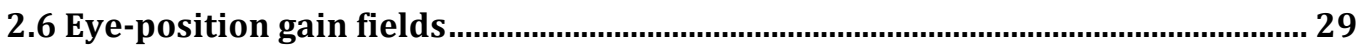

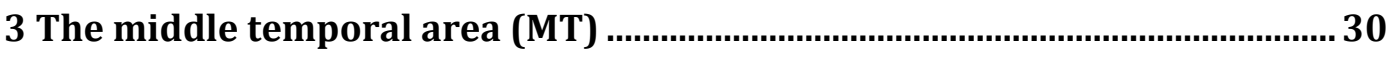

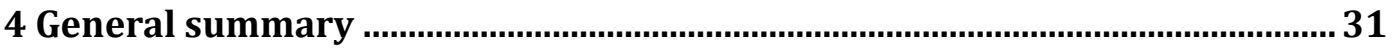

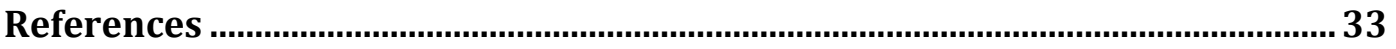

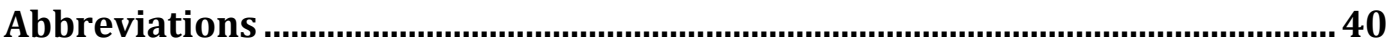


Visual information is the most important information for humans and many other primates. It plays a vital role in the survival and general daily life of primates. However, the brain's capability to process information is limited and it would be overwhelming if all of the visual information in the visual field is processed equally. Several selection mechanisms are used to optimize and prioritize visual information processing. Covert selective attention and foveation are two of the most important selection mechanisms. Covert selective attention enhances the salience of spatial location, objects, or features in the peripheral visual field. Foveation directs the fovea, the region with the highest resolution on the retina, towards the most important and behaviorally relevant objects. Saccades, a type of fast eye movement, are executed to foveate among different objects in the environment and scrutinize them with overt attention. Both covert selective attention and foveation are used to select and optimize the information to be processed by the limited neural resources while subjects are exploring the environment and/or performing visual guided actions. Understanding the relationship between the two selection mechanisms is an important area of research in neuroscience. In this introduction, I will first introduce saccades and covert visual spatial attention, as well as the relationship and the cooperation between the two. Afterwards, I will focus on the trans-saccadic information processing named remapping, introducing its properties, mechanisms, and possible functions.

\section{Spatial attention and its relationship to saccade}

\subsection{Attention and its functions}

"Everyone knows what attention is. It is the taking possession by the mind in clear and vivid form, of one out of what seem several simultaneously possible objects or trains of thought...It implies withdrawal from some things in order to deal effectively with others, and is a condition which has a real opposite in the confused, dazed, scatterbrained state."

-William James, 1890 
This is how William James, one of the leading psychologists and philosophers in nineteenth century, labeled as the 'Father of American psychology', defined attention in his work 'Principles of Psychology'. He implied that attention was a process, which selects which information to be prioritized. Attention is important to our survival, because our information processing capacity is limited, and the amount of sensory information to be processed is huge, especially visual information. If we could not use attention to select the information that we need, we would be in a 'confused, dazed, scatterbrained state' as James mentioned above ((James, 1890), page: 403). In daily life, we normally only focus our attention on one thing. Attention is classified into two types depending on whether the attended location and the gaze location overlap. If the attended location overlaps with the gaze location, it is called overt attention; if the attended location is in the peripheral visual field other than the gaze location, it is called covert attention. In addition, attention can be controlled by external or environmental stimuli, such as onset, flash, or sudden change of a stimulus; or, by internal states such as goals, rewards and tasks. These are called exogenous attention and endogenous attention respectively, or bottom-up and top-down attention (Posner, 1980). Bottom-up attention is attracted by the stimulus saliency, and is considered to be automatic and reflexive, it often induces a reflexive saccade (Friesen and Kingstone, 2003; Mayer, Dorflinger, Rao et al., 2004). In contrast, top-down attention is thought to be voluntary. Visual attention is also classified into spatial attention, objectbased attention and feature-based attention. Spatial attention selects a specific region of the space to attend; everything including the objects and features in this region are attended, whereas object and feature-based attention select a single or a few objects and features to attend irrelevant of the spatial location (Posner, 1980; Tootell, Hadjikhani, Hall et al., 1998). In this thesis, I will focus on top-down, spatial covert attention, which is the best-studied type of attention.

Spatial attention has been likened to a spotlight (Posner, 1980) that enhances information processing in the brain at the attended location. The size of the attended location can be adjusted voluntarily, but the processing efficiency for given sub-location may decrease when the size of the attended location increases (Castiello and Umilta, 1990; Eriksen, Webb and Fournier, 1990). Previous studies have shown that directing attention to a region will improve performance if the task-relevant stimulus is in that region. Some studies showed that not only performance but also appearance is improved by attention (Carrasco, Ling and Read, 2004). 
On the neuronal level, the modulation of spatial attention has been found in almost all visual and visuomotor areas in the brain by recording single neuron signals when the animals are trained to respond to a target. The target is either in the neuron's receptive field (RF), a special region of the visual space in which a visual stimulus will result in a response of that neuron, or out of the recorded neuron's RF in different trials. Frontal and parietal cortical areas such as frontal eye field (FEF) and lateral intraparietal area (LIP) have been reported to be particularly well involved in attentional modulation, some neurons in these areas not only showed higher response when there was a task relevant stimulus in their RF, some of them also controlled the attention enhancement in the lower brain areas (such as V1, MT, V4 etc.) (Goldberg, Bisley, Powell et al., 2006; Wardak, Olivier and Duhamel, 2011; Rao, DeAngelis and Snyder, 2012). In most cases (not always), the firing rate will be higher when the task relevant or target stimulus is located in the neuron's RF compare to when there is a task irrelevant or distracter stimulus in the neuron's RF (Moran and Desimone, 1985; Treue and Maunsell, 1996; Luck, Chelazzi, Hillyard et al., 1997). Attention not only modulates the neuronal firing rate(Petersen, Robinson and Morris, 1987; Treue and Martinez Trujillo, 1999; Ignashchenkova, Dicke, Haarmeier et al., 2004; Buschman and Miller, 2007; Buffalo, Fries, Landman et al., 2010; Rao, DeAngelis and Snyder, 2012); it also increases the gamma-band LFP (local field potential) power(Fries, Womelsdorf, Oostenveld et al., 2008; Gregoriou, Gotts, Zhou et al., 2009), local and cross-areal gamma-band coherence(Saalmann, Pigarev and Vidyasagar, 2007; Gregoriou, Gotts, Zhou et al., 2009), and decreases the neuronal response variability (fano factor) (Mitchell, Sundberg and Reynolds, 2007; Cohen and Maunsell, 2009), low frequency synchrony (Fries, Womelsdorf, Oostenveld et al., 2008) and correlated noise at low frequencies (Cohen and Maunsell, 2009). In addition, attention also modulates properties (such as size, shape, location of peak response etc.) of a neuron's RF ((Womelsdorf, Anton-Erxleben, Pieper et al., 2006; Anton-Erxleben, Stephan and Treue, 2009); for a review see (Noudoost, Chang, Steinmetz et al., 2010)).

\subsection{Saccade}

A saccade is a quick, simultaneous, coordinated movement of both eyes between two phases of fixation. Saccades bring objects or locations onto the foveal part of the retina, where the sharpest visual accuracy is found, to allow better analysis and processing of the 
corresponding information. Saccades are the fastest eye movement and typically occur several times each second (Snodderly, 1987). Peak velocity is from tens up to 800 angular degrees per seconds and duration ranges from 10 to $100 \mathrm{~ms}$ depending on the amplitude of the saccade (Bahill, Bahill, Clark et al., 1975). This high velocity minimizes the time between two fixations and leaves more time to analyze visual information arising from stable fixation. Brain areas such as FEF, LIP, superior colliculus (SC) are highly involved in the preparation and control of saccadic eye movements. For example, some neurons in these brain areas change their responses before a saccade, and are tuned to different saccadic parameters such as saccade direction, amplitude, velocity etc. (Pierrot-Deseilligny, Rivaud, Gaymard et al., 1995; Gaymard, 2012).

\subsubsection{Saccadic suppression}

As mentioned above, saccades rapidly and frequently displace the images on the retina, which should produce a strong blur of the scene when natural viewing of the environment. However, we do not see such blur, instead we perceive a clear and stable world. The blurred images on the retina therefore seem to be suppressed, and this suppression of visual information processing is called saccadic suppression (Bridgeman, Hendry and Stark, 1975; Volkmann, 1986). Psychophysical studies have found saccadic suppression shortly before and during saccades, such as elevated detection thresholds for visual flashes (Zuber and Stark, 1966), motion changes (Burr, Holt, Johnstone et al., 1982), object displacement (Bridgeman, Hendry and Stark, 1975) etc. But saccadic suppression has been found to be absent or weak in some tasks, for example, detecting low spatial frequency patterns and in color discrimination tasks (Burr, Holt, Johnstone et al., 1982; Burr, Morrone and Ross, 1994; Diamond, Ross and Morrone, 2000), which suggests saccade suppression affect differently on different tasks that may relate to different pathways in the brain. In a discrimination task, Diamond and colleagues found that saccadic suppression started at about $50 \mathrm{~ms}$ before saccade onset and lasted for another $50 \mathrm{~ms}$ after saccade offset (Diamond, Ross and Morrone, 2000). Electrophysiological results also reported saccadic suppression in visual brain areas, such as LGN (lateral geniculate nucleus), V1, V2, V3, V4, MT (middle temporal), MST (medial superior temporal), pulvinar, SC etc. (Robinson and Wurtz, 1976; Reppas, Usrey and Reid, 2002; Thiele, Henning, Kubischik et al., 2002; Sylvester and Rees, 2006; Ibbotson, Price, Crowder et al., 2007; Kagan, Gur and Snodderly, 2008; Berman and Wurtz, 
2011; Hass and Horwitz, 2011; Zanos, Mineault, Guitton et al., 2016). These visual areas are not found to participate in saccade control, however, the response are significantly suppressed by saccade.

The mechanisms underlying saccadic suppression are not yet well understood. Pre- and post-saccadic visual masking and corollary discharge/efference copy signal have been proposed to explain saccade suppression (Ross, Morrone, Goldberg et al., 2001; Wurtz, 2008; Wurtz, McAlonan, Cavanaugh et al., 2011). Visual masking normally means the perception of a visual target stimulus is impaired by the presence of a mask stimulus; the mask stimulus could be presented shortly before or after the target stimulus. The visual masking theory suggests saccadic suppression results from forward and backward masking effects of pre- and post-saccadic visual stimuli. Saccadic suppression is absent or weak when only the target stimulus is presented during a saccade and no other stimuli are displayed before and after saccade (Deubel, Elsner and Hauske, 1987; Castet and Masson, 2000; Castet, Jeanjean and Masson, 2002). Wurtz and colleagues suggest that a corollary discharge (CD) - an extra-retinal signal or an internal copy containing impending saccadic eye movement commands (Sperry, 1950; Matthews, 1982), plays an important role in saccadic suppression. An important piece of evidence supporting this theory is the observation that saccadic suppression precedes the onset of saccades (Wurtz, 2008; Wurtz, Joiner and Berman, 2011; Wurtz, McAlonan, Cavanaugh et al., 2011). An electrophysiological study in non-human primates also suggested that corollary discharge might contribute to the saccade suppression. When the eye muscles were paralyzed (the monkeys were unable to execute saccades), but the monkeys were trained to try making saccades, the background responses of neurons in V1 were suppressed (Judge, Wurtz and Richmond, 1980). However, in a behavioral experiment with human subjects viewing natural scenes, Dorr and Bex found that saccadic suppression could be explained by the high speed of the moving image on the retina caused by a saccade. They concluded that saccadic suppression during natural vision does not have to derive from an active extraretinal mechanism but can result only from the blur caused by the fast eye movement (Dorr and Bex, 2013). However, the authors did not explain why saccade suppression could happen before saccade onset. 


\subsection{Relationship between spatial attention and saccades}

Saccades bring relevant and important objects or locations to the fovea from peripheral visual locations to facilitate their processing; attention selects which information should be processed and guides saccade. The relationship between attention and saccades is mutually beneficial when the saccade and attention target overlap. It is possible that saccade target selection and attention target selection share the same neural mechanisms. Previous studies have provided abundant evidence for overlapped neural mechanisms that control saccade and spatial attention (Kowler, 2011). For example, previous studies have revealed that brain areas FEF, LIP and SC are highly involved in both attention modulation and saccade control. Some psychophysical evidence has demonstrated the impact of covert orienting of visual spatial attention on saccades. For example, there are studies showing that deviation of saccade trajectories depends on the attended location (Rizzolatti, Riggio, Dascola et al., 1987). A similar psychophysical study also demonstrated that covert spatial attention perturbed saccade preparation or programming (Kowler, Anderson, Dosher et al., 1995).

Saccade preparation has also been reported to show influence on attention and on subjects' performance. For example, in a study, the subjects were asked to make a saccade to a peripheral location while detect a visual stimulus presented before the saccade, the authors found that detection accuracy was highest when the visual target was located at the same location as the saccade target. In addition, when the visual target and saccade target were mismatched (i.e. not at the same location), detection accuracy was impaired compared to the detection-only task (without a saccade). The authors suggested that spatial attention was involved in saccade programming and/or execution (Hoffman and Subramaniam, 1995). Similar results were reported in other psychophysical studies (Deubel and Schneider, 1996; Deubel, 2008). On the neuronal level, several studies have shown that when there was a saccade target in the neuron's RF, the neuron's response was enhanced before saccade as if saccade targets attracted attention automatically, which suggests an influence of saccade preparation on attention (Gottlieb, Kusunoki and Goldberg, 1998; Moore, Tolias and Schiller, 1998; Steinmetz and Moore, 2014). Microelectrical or TMS stimulation of oculomotor areas such as FEF and SC improved animal and human subjects' performance (Grosbras and Paus, 2002; Cavanaugh and Wurtz, 2004; Moore and Fallah, 2004; Chambers and Mattingley, 2005; Muller, Philiastides and 
Newsome, 2005) as well as modulated the response of lower visual hierarchical areas (Moore and Armstrong, 2003). These results support the 'premotor theory of attention', which proposes shared neuronal mechanisms for saccade preparation/programming and covert spatial attention (Rizzolatti, Riggio, Dascola et al., 1987; Corbetta, 1998; Corbetta, Akbudak, Conturo et al., 1998).

But other studies have argued against the 'premotor theory' (Morgan, Ball and Smith, 2014). It is possible that these two systems are parallel but distinct; they just act in a similar way. Some physiological studies performed in monkeys have investigated the relationship between the two systems by perturbing neural signals in one system while examining the other at the same time. For example, by using micro-stimulation in SC or FEF, and simultaneously recording from neurons in other visual areas like MT or V4, it is possible to address the causal relationships of neural signals between the oculomotor system and spatial attention system. Some studies have confirmed that different functional roles were played by distinct neuronal subgroups in FEF, SC and LIP (Ignashchenkova, Dicke, Haarmeier et al., 2004; Thompson, Biscoe and Sato, 2005; Brown, Hanes, Schall et al., 2008). Visual neurons, visuomotor neurons and motor neurons are coexistent in brain areas of LIP, SC and FEF. Visual neurons respond only to the visual stimuli presented in their RF; motor neurons respond only to eye movements or/and limb movements to the neuron's response field; visuomotor neurons respond to both visual stimuli and eye/limb movements. Thompson and colleagues found that when monkeys were doing a covert attention task without eye movement, the responses of visual neurons and visuomotor neurons were enhanced, whereas the motor neurons were not enhanced, even showing inhibition in FEF (Thompson, Biscoe and Sato, 2005). A similar observation was reported by recording single neurons from SC when monkeys performed a covert spatial attention task. The results showed that only the visual and visuomotor neurons were enhanced by attention, but not motor neurons in SC (Ignashchenkova, Dicke, Haarmeier et al., 2004). Other studies also confirmed that different functional roles were played by distinct neuronal subgroups (Brown, Hanes, Schall et al., 2008). Moreover, inactivation of LIP did not impair saccade parameters but indeed impaired the animal's visual search task (Wardak, Olivier and Duhamel, 2002, 2004). The authors suggested that LIP 'is not involved in the saccadic execution per se', but is involved in processes such as salience representation (Wardak, Olivier and Duhamel, 2011). 
The psychophysical studies and stimulation studies mentioned above suggest shared mechanisms behind covert spatial attention and saccade preparation. However, single neuron data and inactivation studies suggest a dissociation of the two. Visual neurons are more involved in selecting the visual target and motor neurons are more involved in saccade programming. More studies are needed in the future to better understand the mechanisms behind the two.

\subsection{Shifting of spatial attention}

In daily life, spatial attention typically overlaps with where we are looking, which means that attention modulates the neurons with foveal receptive fields. In this situation, spatial attention does not have to modulate different neurons in the brain; instead, we make saccades to select different targets when we view our environment. However, attention does not always modulate foveal neurons. There are at least two processes requiring attention shifts between different groups of neurons. One is when subjects are fixating at one location and shift attention among different objects in the peripheral visual field. For example, when we are driving towards an intersection, we shift attention from side to side while looking at the vehicles before ours. Attention has to shift from one group of neurons to another (the neurons with left RFs to neurons with right RFs for example). The other situation is when the target object is stable while our eyes, head and/or body is moving, which means the target images on the retina are changing, resulting in the need for an attention shift among neurons in the brain. In the following section, I will review the existing literature of attention shift studies related to these two situations.

\subsubsection{Attention shift with fixation}

It is possible and common that human and non-human primates covertly shift attention between different locations in the visual field while keeping the eyes at fixation. Several studies have investigated covert spatial attention shifts in visual areas such as LIP and MT. In a study recording from MT neurons, Busse and colleagues (2008) used a double-cueing paradigm. The monkeys were trained to covertly attend to one target random dot pattern (RDP) out of three RDPs, indicated by the first exogenous cue. In one experimental condition, the monkeys had to endogenously/voluntarily covertly shift their spatial attention to another of the three RDPs, indicated by the second cue. By manipulating the 
combination of the first and second cued RDP locations, spatial attention could shift into or out of the recorded neuron's RF. The results suggested that the exogenous attention induced by the onset of the cue took about $120 \mathrm{~ms}$ to affect the MT neuron's response. The latency of endogenous attention shifting into the neuron's RF was about $190 \mathrm{~ms}$ after the second cue onset. Finally, the authors found that the disengagement time of spatial attention from the recorded neuron's RF was about $300 \mathrm{~ms}$ after the cue shifting the monkey's attention out of the neuron's RF (Busse, Katzner and Treue, 2008).

Herrington and Assad used a similar paradigm to investigate the temporal dynamics of covert attention shifts in LIP and MT. They found that LIP neurons were significantly enhanced earlier than MT neurons after cue onset when the attention was shifted into the neuron's RF. It took about 200ms (median: 166ms for one monkey, 230ms for another) in LIP and $250 \mathrm{~ms}$ in MT (median: $228 \mathrm{~ms}$ for one monkey, $280 \mathrm{~ms}$ for another). Similar to the above study, they found that attention also took longer (305ms in LIP; $348 \mathrm{~ms}$ in MT) to fade away when attention was shifted out of the neuron's RF compared with the speed of attention engagement in both brain areas (Herrington and Assad, 2009, 2010).

These studies, along with a study recording neurons in V1 (Khayat, Spekreijse and Roelfsema, 2004), suggest that when the animals are trained to shift attention while maintaining eye fixation, the engagement of attention modulation takes more than $100 \mathrm{~ms}$ after cue onset, and it is faster than disengagement in visual areas. In addition, the studies confirm that covert attention shifts are a top-down process initiating from parietal or frontal areas and then transfering to lower visual areas like MT. The studies also support the theory that the origin of attention is in parietal or frontal cortex.

Previous studies suggest that it takes about one hundred milliseconds to several hundred milliseconds to covertly shift attention modulation from one neuron to the other when the eyes maintain fixation. However, attention shifts across saccades among neurons seems to use a different mechanism to covert attention shifts. Some studies suggested that the attention modulation might shift to other neurons before a saccade in the form of 'attention pointers' (Cavanagh, Hunt, Afraz et al., 2010c). We will discuss this issue in the later part. 


\subsubsection{Attention shift across saccade}

The other situation in which spatial attention benefit has to shift across neurons in retinotopic visual areas is when the target object is stable and the subject is making eye or head movements. In this case, the target will be transferred across saccades from RFs of one group of neurons to another. Because spatial attention only benefits neurons whose RFs cover the target, spatial attention benefit transfers from the neurons whose RFs cover the target before the saccade to the neurons with RFs covering the target after the saccade. Therefore, in retinotopic areas, attention modulation has to be reestablished in one group of neurons, and fade away from another group.

In a physiological study, Khayat and colleagues investigated the reestablishment of objectbased attention modulation after a saccade. Two curves were displayed on the screen after a short fixation. The monkeys were required to make two saccades along the target curve and ignore the distractor one. Multi-unit activity in V1 was recorded while the monkeys were doing the task. In one condition, part of the target curve was brought into the RF of the multi-units by the first saccade. The results showed that the attentional enhancement was reestablished rapidly after the first saccade. It is about $50 \mathrm{~ms}$ faster than when the target curve was directly displayed in the RF of the multi-units. The authors suggested that the results might indicate remapping of attention (Khayat, Spekreijse and Roelfsema, 2004). However, pre-saccadic remapping was not found in V1. The authors suggested that the attention enhancement in V1 might have been gated by the visual stimulus (Khayat, Spekreijse and Roelfsema, 2004). Because there was no stimulus in the units' RF before the first saccade, it is impossible to quantify the attention enhancement if the attentional modulation arrived in V1 before the first saccade. Moreover, they did not investigate the time course of how attention faded away after a saccade when the target was brought out of the neuron's RF. Whether object-based attention and spatial attention share the same neuronal mechanisms remains unknown. In this thesis, we use a spatial attention paradigm; and try to demonstrate the complete dynamics of attention shifts across saccade in visual area MT.

Attention shifts across saccades is highly related to a phenomenon called trans-saccadic remapping (Cavanagh, Hunt, Afraz et al., 2010). In the following sections of this introduction, I will review the related psychophysical studies on human and neuronal physiological studies on non-human primates. 


\section{Remapping across saccades}

\subsection{Remapping}

The visual and visuomotor areas are organized in a retinotopic pattern; the adjacent neurons in these brain areas have slightly different RFs. These RFs form an orderly and systematically map covering the visual field. Because of this organized arrangement of the neurons' RFs, the neurons in these areas could be seen as formatting a whole map of the visual field. Each visual neuron in these areas has a RF corresponding to a region on the retina, and the neuron only responds to the stimulus located inside its RF. When the eyes are moving, the RF of a specific neuron therefore corresponds to different spatial locations in the environment, directly dependent on the current location of the eyes. However, some neurons in visual or visuomotor areas, such as LIP, FEF, SC, also respond to a flashed visual stimulus at the location that an upcoming saccade will bring into the RF. Importantly, the stimulus is not in the neuron's classical retinotopic RF before or after saccade, it only flashes briefly before saccade onset in the neurons' post-saccadic RFs. It seems therefore that these neurons either predict the occurrence of a stimulus will be brought into the RF by the upcoming saccade, or they remember there was a stimulus appearing before the last saccde in the post-saccadic RF location. This peri-saccadic neuronal activity, which the neurons will change their responses before or after saccade when a stimulus flashed in the post-saccadic RF, is called remapping. It has been proposed as an important mechanism for visual stability and keeping track of objects across saccades (Wurtz, 2008; Burr and Morrone, 2011; Hall and Colby, 2011; Mathot and Theeuwes, 2011; Melcher, 2011; Wurtz, Joiner and Berman, 2011; Mirpour and Bisley, 2012; Higgins and Rayner, 2015; Rolfs, 2015; Marino and Mazer, 2016). Two types of remapping are distinguished regarding the temporal dynamics of the activity: 1) predictive remapping, and 2) post-saccadic memory traces. In the first case, when a stimulus was brought into a neuron's RF, this neuron will change its response before the saccade is executed, or the latency of the evoked response is shorter than the normal visual response when a stimulus appears in the neuron's RF. On the other hand, when the evoked response occurs only after the saccade, and has longer latency than the normal visual response, this response is referred as post-saccadic remapping.

However, the research results regarding the spatial dynamics (i.e., receptive field dynamics) of remapping responses are inconsistent (Melcher, 2011). Some studies suggest a jump of 
the RF from the pre-saccadic RF to post-saccadic RF (Wurtz, Joiner and Berman, 2011); some suggest that the RF shifts towards the saccade target (Zirnsak and Moore, 2014; Zirnsak, Steinmetz, Noudoost et al., 2014); some suggest both of the above shifts (Neupane, Guitton and Pack, 2016); and some suggest expansion of the RF (Wang, Fung, Guan et al., 2016). Therefore, the exact spatial properties of remapping require more investigation.

\subsubsection{Physiological studies}

The first descriptions of remapping in single-neuron recording studies were reported in FEF (Goldberg and Bruce, 1990) and LIP (Barash, Bracewell, Fogassi et al., 1991) using the double-step paradigm. This paradigm requires the monkeys to make two successive saccades to two saccade target locations that are briefly presented before the first saccade. The disappearance of the saccade targets signals the movement of the eyes. In this paradigm, the subjects have to prepare both of the upcoming saccades before the first saccade. The authors found that if the second saccade target was brought into the recorded neuron's RF by the first saccade, neurons showed higher responses, even though the saccade targets were removed before the eyes moved, and no visual stimulus was present in the neuron's RF before and after saccade. This was explained as the remapping of the second saccade target, i.e. the neuron responded to the remapped memory trace of the second saccade target (Mathot and Theeuwes, 2011).

In a landmark remapping study (Duhamel, Colby and Goldberg, 1992), the authors recorded neuronal responses in LIP while the trained monkeys made saccades to a saccade target. In one condition, a visual stimulus was presented in the neuron's post-saccadic RF, and no stimulus was presented in the neuron's pre-saccadic RF. They found some LIP neurons had a higher response just before saccade, which was proposed as predictive remapping. In another condition, the visual stimulus was only briefly flashed in the neuron's post-saccadic RF before saccade; thus, there was no stimulus in the neuron's RF before and after saccade. However, some neurons still had a higher response after saccade compared with the simple saccade condition, as if the neurons remembered there was a stimulus in the post-saccadic RF location. The authors proposed this response to reflect the other remapping activity, namely a memory trace (Figure 1). This remapping activity is not a pure visual response, because the neurons do not respond to the stimulus in the postsaccadic RF unless a saccade is made. It is also not a motor response, because the neurons do not show remapping activity when a simple saccade is made. Rather, the remapping 
activity reflects the neurons' expectation that the visual stimulus will be brought into their RF by a saccade (predictive remapping); or, the neurons are 'remembering' that the visual stimulus is in their post-saccadic RF (post-saccadic memory trace). Further properties (spatial and temporal) of remapping activity in LIP were revealed by later studies (Colby, Duhamel and Goldberg, 1996; Kusunoki and Goldberg, 2003; Heiser and Colby, 2006).

a

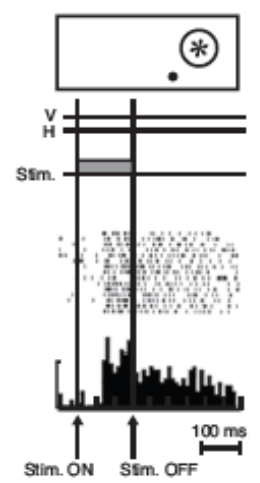

b

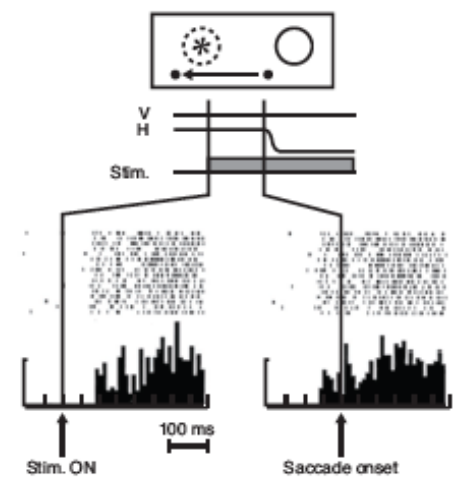

C

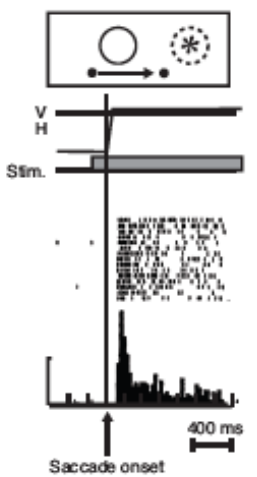

d

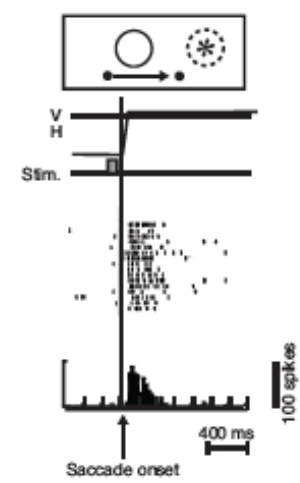

Figure 1. Neuronal remapping in LIP. Top row rectangles show the paradigm used in each condition of the experiment. Dots, star, circle, dashed circle, and arrow indicate the fixation and saccade targets, visual stimulus, pre-saccade RF, post-saccade RF, saccade, respectively. The time lines below the rectangules show the vertical (V) and horizontal $(\mathrm{H})$ eye position, as well as the times in which the visual stimulus was displayed (Stim.). The raster plots show example neurons responses to each condition, each row indicates one trial. Spike density histograms are shown below. The histograms and the raster plots are aligned to the vertical lines indicating events within a trial. The time scales below the histograms indicate $100 \mathrm{~ms}$ (a, b) or $400 \mathrm{~ms}$ (c, d). (a) The example neuron responds to the visual stimulus presented within the RF in a fixation task. The neuron shows a normal visual response. (b) The neuron responds to the visual stimulus that is brought into its RF by a saccade. Data are aligned to stimulus onset in the left panel and to the saccade onset in the right panel. This neuron shows a pre-saccadic predictive remapping response. (c) Another example neuron that do not show a pre-saccadic predictive remapping - the neuron shows no response until after the saccade is made. (d) The same neuron as (c) discharges when a saccade brings a presaccadically flashed visual stimulus into its RF after saccade, even though the stimulus is never present in its RF. This neuron shows a remapped memory trace. (Adapted from Duhamel, Colby and Goldberg, 1992) 
Remapping activity has also been found in other brain areas such as frontal eye field (FEF) (Umeno and Goldberg, 1997, 2001; Sommer and Wurtz, 2004, 2006, 2008), superior colliculus (SC) (Walker, Fitzgibbon and Goldberg, 1995; Dunn, Hall and Colby, 2010; Churan, Guitton and Pack, 2012) medial superior temporal area (MST) (Inaba and Kawano, 2014)and in the ventral stream in areas V4, V3a, V3 and V2 (Tolias, Moore, Smirnakis et al., 2001; Nakamura and Colby, 2002; Neupane, Guitton and Pack, 2016) using similar paradigms. Remapping activity is not identical in different brain areas, though it has been found in the brain areas mentioned above. The variety of the remapping activity reported in these studies showed some trends along the visual hierarchy. First, the proportion of neurons showing remapping activity decreases when moving down the visual hierarchy. For example, nearly all neurons (96\%) in LIP show remapping activity (Duhamel, Colby and Goldberg, 1992), about half (52\%) in V3A, and the proportion drops to 35\% in V3, $11 \%$ in V2 and 2\% in V1 (Nakamura and Colby, 2002). Predictive remapping shows a similar tendency, it was reported in around 44\% of LIP neurons (Duhamel, Colby and Goldberg, 1992) and decreases to 35\% in V3A (Nakamura and Colby, 2002). No predictive remapping was found in MT, V2 or V1 (Nakamura and Colby, 2002; Ong and Bisley, 2011). Second, the strength of remapping activity also decreases down the visual hierarchy (Gottlieb, 2007; Merriam, Genovese and Colby, 2007). Third, the mean latency of neuronal remapping activity relative to saccade onset showed an increase at lower levels of visual hierarchy (Nakamura and Colby, 2002; Higgins and Rayner, 2015).

Remapping is a clear and replicable phenomenon in many brain areas of non-human primates. Given that the detection of remapping requires relatively high spatial (the level of receptive fields) and temporal (ms) resolution, it is challenging to detect remapping in the human brain. Several brain imaging studies in human subjects have reported remapping responses in human brain using non-invasive techniques such as fMRI, magnetoencephalography (MEG) and electroencephalography (EEG). The rationale behind the experimental design is similar to the animal experiment. The stimulus was removed before saccade and transferred from one visual hemifield to the other, and therefore from one brain hemisphere to another, by a saccade. If the condition evoked a different response in the hemisphere ipsilateral to the hemifield of initial stimulus presentation compared to control (pure motor or visual response), it was judged as evidence for remapping. 
For example, in an fMRI study, a visual stimulus was presented in one visual field (left hemifield for example) only before saccade, and the subjects were asked to make a left saccade beyond the stimulus. The stimulus would then be located in the right visual hemifield after the saccade. If the task evoked a change in BOLD (blood-oxygenation level dependent) signal of the left hemisphere after saccade compared to control conditions, it is interpreted as a remapping signal because there was no stimulus present in the corresponding visual field (right in this case) at any time across the trial. The authors indeed found such remapping BOLD signals in parietal cortex (Merriam, Genovese and Colby, 2003), in line with the results from the monkey electrophysiology experiments described above. Medendorp and colleagues also found BOLD and gamma-band (in MEG signal) remapping signal in parietal cortex using a double-step task (Medendorp, Goltz, Vilis et al., 2003; Medendorp, Goltz and Vilis, 2006; Van Der Werf, Jensen, Fries et al., 2008). Similar remapping signals were found in visual cortex from V1 to V4 using a similar paradigm (Merriam, Genovese and Colby, 2007). The results also suggested that the strength of remapping decreases along the visual hierarchy from high to low areas, which is consistent with electrophysiological data from monkeys (Nakamura and Colby, 2002; Merriam, Genovese and Colby, 2007). MT has also been reported to show remapping in an fMRI experiment (d'Avossa, Tosetti, Crespi et al., 2007). However, the low temporal resolution of fMRI makes it difficult to find a predictive remapping signal. EEG has been used to investigate predictive remapping in humans using similar experimental design to the studies mentioned above because of its higher temporal resolution. However, most studies failed to find any predictive remapping signal (Bellebaum, Hoffmann and Daum, 2005; Bellebaum and Daum, 2006; Parks and Corballis, 2008; Peterburs, Gajda, Hoffmann et al., 2011). The exception was one EEG study that found signal enhancement before saccade onset in some electrodes above the parietal cortex (Parks and Corballis, 2010).

\subsubsection{Psychophysical studies}

As demonstrated above, the strongest evidence for remapping has been found in experiments using extracellular recording in monkey brain. Psychophysical studies have also been performed to investigate the behavioral consequences of remapping in human subjects. Most of these studies used adaptation paradigms such as the tilt after-effect (TAE), motion after-effect (MAE), or face emotion adaptation. The logic underlying these experiments is that adaptation effects are often strongest when adaptor and test stimuli 
are presented at the same retinotopic location (Dickinson, Mighall, Almeida et al., 2012). If remapping transfers feature information across a saccade, then an adaptation effect should be detectable at the remapped location. For example, in one study (Melcher, 2007) the subjects were asked to distinguish the tilt of a test grating (test) after adaptation by an adapting grating (adaptor) while preparing a saccade. In the important conditions, the adaptor was placed at the pre-saccadic initial fixation point (foveal region), and the test was presented either at the same location as the adaptor (the initial fixation point, i.e. the same spatiotopic location) or the location of the saccade target (i.e. the same retinotopic location). The test could be shown before or after saccade (Figure 2). The results showed that the TAE gradually increased before saccade onset when the test was placed at the saccade target location; at the same time, the TAE gradually decreased before saccade onset when the test was presented at the adaptor location (initial fixation location) even through the adaptor and the test were at the same retinotopic locantion. Together with other TAE studies (Melcher, 2005, 2009; Zimmermann, Morrone, Fink et al., 2013), the results suggested a transfer of the feature information just before saccade. Besides, in another experiment of the same study (Melcher, 2007), when the adaptor was shown at the initial fixation location, and the test was placed at an intermediate position between the initial fixation point and saccade target, human subjects also display a TAE before the saccade, which suggested remapping towards the saccade target or an expansion of neurons' RFs across saccade as mentioned in the physiological studies. MAE was also reported at the same spatiotopic location across saccades using a similar paradigm (Ezzati, Golzar and Afraz, 2008; Biber and Ilg, 2011; Turi and Burr, 2012; Yoshimoto, Uchida-Ota and Takeuchi, 2014). As well as TAE and MAE, Melcher also reported spatiotopic aftereffects for face, tilt and shape adaptation (Melcher, 2005). A face emotion after-effect after emotional adaptation at the same spatiotopic location after saccade has also been reported (Wolfe and Whitney, 2015).

However, the overall results of these psychophysical studies were inconsistent, and sometimes even contradictory. Some studies found the TAE only at the same retinotopic location but not the same spatiotopic location across saccades (Mathot and Theeuwes, 2010, 2013). Similarly inconsistent results were also reported for the MAE (Knapen, Rolfs and Cavanagh, 2009; Turi and Burr, 2012), face aftereffect (Afraz and Cavanagh, 2008), and direction aftereffect (Wenderoth and Wiese, 2008). 


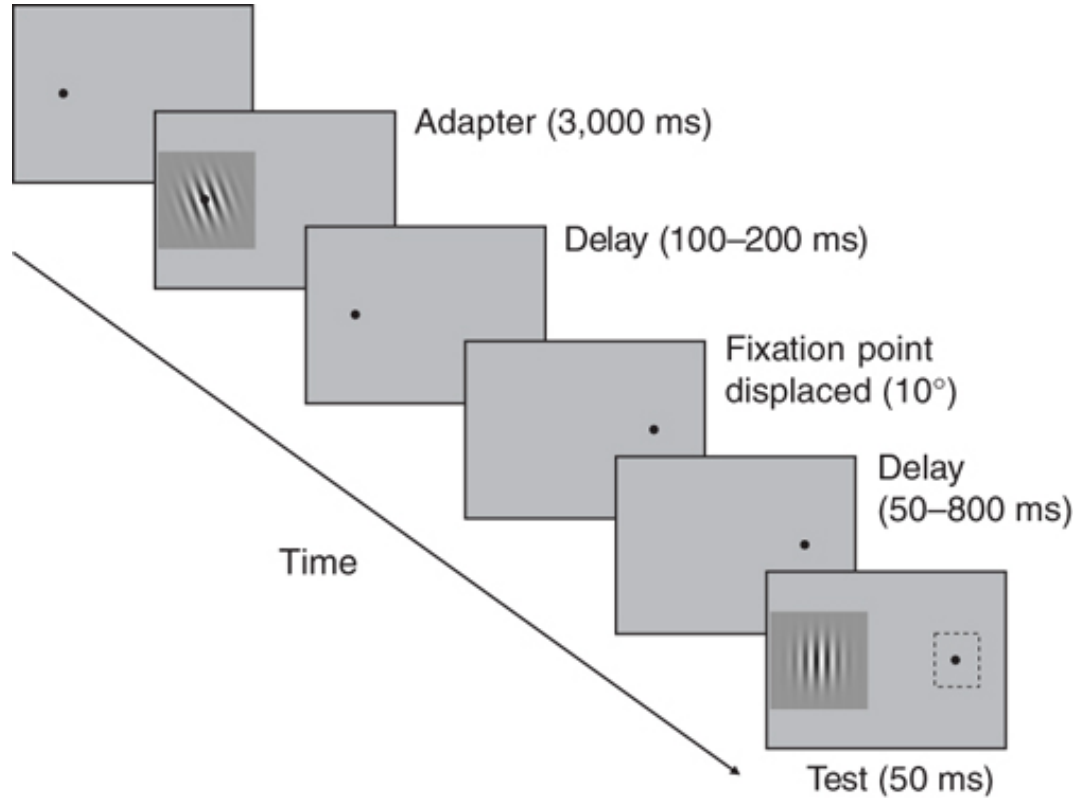

Figure 2. The paradigm of a TAE experiment. The grating indicates the visual stimuli, the dots indicates the fixation point and saccade target. After a short fixation, an adapter stimulus is shown at the fixation point for 3,000 ms. After a short delay, the fixation point jumps 10 degrees to the other side of the screen, which cues the subjects make a saccade to the new location. A test stimulus is shown for $50 \mathrm{~ms}$ after $50-800 \mathrm{~ms}$ of fixation point jumping, which allows the test could be shown before or after the saccade, the test stimulus is displayed either at the pre-saccadic fixation point (as the figure shows) or location of the post-saccadic fixation point (dashed square). (Adapted from Melcher, 2007)

For example, Afraz and Cavanagh only found retinotopic face aftereffects but not spatiotopic (Afraz and Cavanagh, 2008); and Mathot and Theeuwes reported the TAE was found only when the adaptor and the test were at the same retinotopic location, but not when the adaptor and the test shared the same spatiotopic location across saccade (Mathot and Theeuwes, 2013). Given these negative results on the spatiatopic aftereffect, Cavanagh and colleagues suggested that feature properties were not remapped across saccades; they suggested that remapping referred instead to a transfer of the attentional pointer in retinotopic brain areas (Cavanagh, Hunt, Afraz et al., 2010c; Cavanagh, Hunt, Afraz et al., 2010a). Others argued that the apparent spatiotopic integration of motion information across the saccade resulted noise in the perceptual system regarding the time of the 
motion stimulus onset related to the saccade, and claimed that it might not have any practical function (Morris, Liu, Cropper et al., 2010).

On closer examination, there are some factors that might explain part of the variability in the psychophysical studies, for example, the differences in experimental method or/and stimuli used in the experiments. It seems that drifting Gabors and gratings were less likely (Wenderoth and Wiese, 2008) than random dot patterns (RDP) to elicit the MAE at spatiotopic locations (Ezzati, Golzar and Afraz, 2008; Wenderoth and Wiese, 2008; Knapen, Rolfs and Cavanagh, 2009; Turi and Burr, 2012). How the aftereffect was quantified might also be of importance. In different studies, different baseline and control conditions were used, which might result in inconsistencies (Marino and Mazer, 2016). Finally, the different duration between the test stimulus and saccade onset might contribute to the difference of the results (Turi and Burr, 2012).

In sum, based on current evidence it is unclear whether or not remapping transfers feature information across a saccade (spatiotopic aftereffect) in humans and in which cortical areas such a transfer might occur. For future studies, experimental methods, stimulus type, baseline conditions, and stimulus presentation timing should be well controlled to allow better comparison of the results of remapping in human subjects.

\subsection{Corollary Discharge and remapping}

Although remapping activity has been found in many areas of nan-human primate cortex, the underlying neural mechanisms and circuits are not well understood. Wurtz and colleges (Sommer and Wurtz, 2002, 2006, 2008; Wurtz, 2008; Wurtz, Joiner and Berman, 2011) proposed that the corollary discharge ( $C D$, or efference copy), an internal copy containing impending saccadic eye movement commands (Sperry, 1950; Matthews, 1982), might contribute to remapping activity. SC was suggested as an ideal source of the CD because of the retinotopic map it contains and the direct control of eye movement. The CD originating from SC would likely contain information about the direction, amplitude, and velocity of the planned eye movement, and could be transfered to the cortex and act as remapping activity. Furthermore, they proposed the medial dorsal (MD) nucleus of the thalamus, an oculomotor area between motor area SC and visuomotor area FEF, as an important relay to transfer the CD from SC to cortex. In the seminal study of Sommer and Wurtz (2002), MD neurons were recorded when the monkeys were making simple 
saccades. The results showed that the MD neurons responded before saccade onset, while inactivation of the MD did not change the saccade vector in speed, direction or amplitude. Both findings were consistent with CD signal. In a further condition, the monkeys were trained to perform a double-step saccade towards saccade targets that were removed before the first saccade onset. The disappearance of the saccade target before saccade onset forced the brain to take into account the vector of the first saccade to perform the second saccade correctly. The results of this condition showed that after inactivation of one side of the MD nucleus, the second saccade was impaired when the saccade targets were located in the contralateral visual field, which meant that the brain failed to take into account the vector of the first saccade (lost the CD signal) when preparing the second one. These results support a role of MD in relaying the CD signal.

In a subsequent study (Sommer and Wurtz, 2006), FEF neurons were recorded while the trained monkeys made a saccade, and a probe was flashed around the time of saccade either in the neuron's pre-saccadic or post-saccadic RF. Both predictive remapping and a memory trace were found in FEF. The authors also found that the visual responses of some neurons in FEF to the probe became weaker when it was flashed in the pre-saccadic RF just before a saccade, while the responses (remapping activity) became stronger when the probe was flashed in the post-saccadic RF at the same time. However, when the probe was flashed at the midpoint between pre-saccadic RF and post-saccadic RF, the neuron was unresponsive. This result suggested that the neuron's RF shifted rather than spanned the space to the post-saccade RF, which is in line with the predictions of the CD. More importantly, the authors found that the remapping activity in FEF was impaired after inactivation of MD by injection of muscimol, a selective agonist of GABA receptors. The authors thus suggested that the CD from the SC-MD-FEF pathway was necessary and sufficient to induce RF shifting (remapping) in FEF neurons. This demonstration provided a potential circuit-level explanation of how the corollary discharge originating from SC is transferred to cortex, and emerges as remapping.

The above findings provided a possible neuronal mechanism for remapping, and showed that MD inactivation impaired both the remapping response in FEF and the motor planning of the saccade in a double-step task. However, whether the SC-MD-FEF circuit directly affects the monkey's visual perception is not clear. If MD indeed relays the CD signal from SC to frontal cortex, inactivation of MD may impair the visual perception of the subject's 
own saccade vector, but the saccade itself should be not influenced. This was found in a recent study (Cavanaugh, Berman, Joiner et al., 2016). The monkeys were trained to make a saccade to a target located on the left or right of fixation, but the target may jump a small distance (less than two degrees back or forth) during the execution of the saccade. The monkeys had to report the direction of the target displacement by moving a bar after saccade. The perception of the saccade end point (the report of the target displacement) and the actual saccade end point were decoupled by this paradigm. The authors found that, during unilateral MD inactivation with muscimol injection, the perception of the saccade towards the contralateral side was impaired, but the saccade itself was not affected as predicted. The authors therefor concluded that the CD signal containing internal information contributed to the perceived visual stability created by the brain. An earlier case study of a human patient with MD lesions reported a similar impairment in a doublestep experimental paradigm, which strongly suggests that humans share the same CD circuit and function with monkeys (Bellebaum, Daum, Koch et al., 2005). These results suggest that the remapping CD not only affects neuronal responses, but also the subject's perception.

\subsection{The role of attention in remapping}

It is well known that attention modulates visual perception (Posner, 1980; Luck and Ford, 1998; Boynton, 2005). Previous studies also showed the influence of attention on remapping (for a review see Mathot and Theeuwes, 2011). In a classic study, Gottlieb and colleagues (1998) found that when a saccade brought a recently onset salient stimulus which was expected to capture exogenous/bottom-up attention into an LIP neuron's RF, the neuron showed higher remapping activity than when a stable stimulus which was presented on the screen for a long time, was brought into the neuron's receptive field. The recorded LIP neurons also showed similarly higher remapping activity when a taskrelevant stimulus (a saccade target in this study) was brought into the RF by a saccade

(Gottlieb, Kusunoki and Goldberg, 1998) (Figure 3). The results underline the importance of attention in remapping activity. 

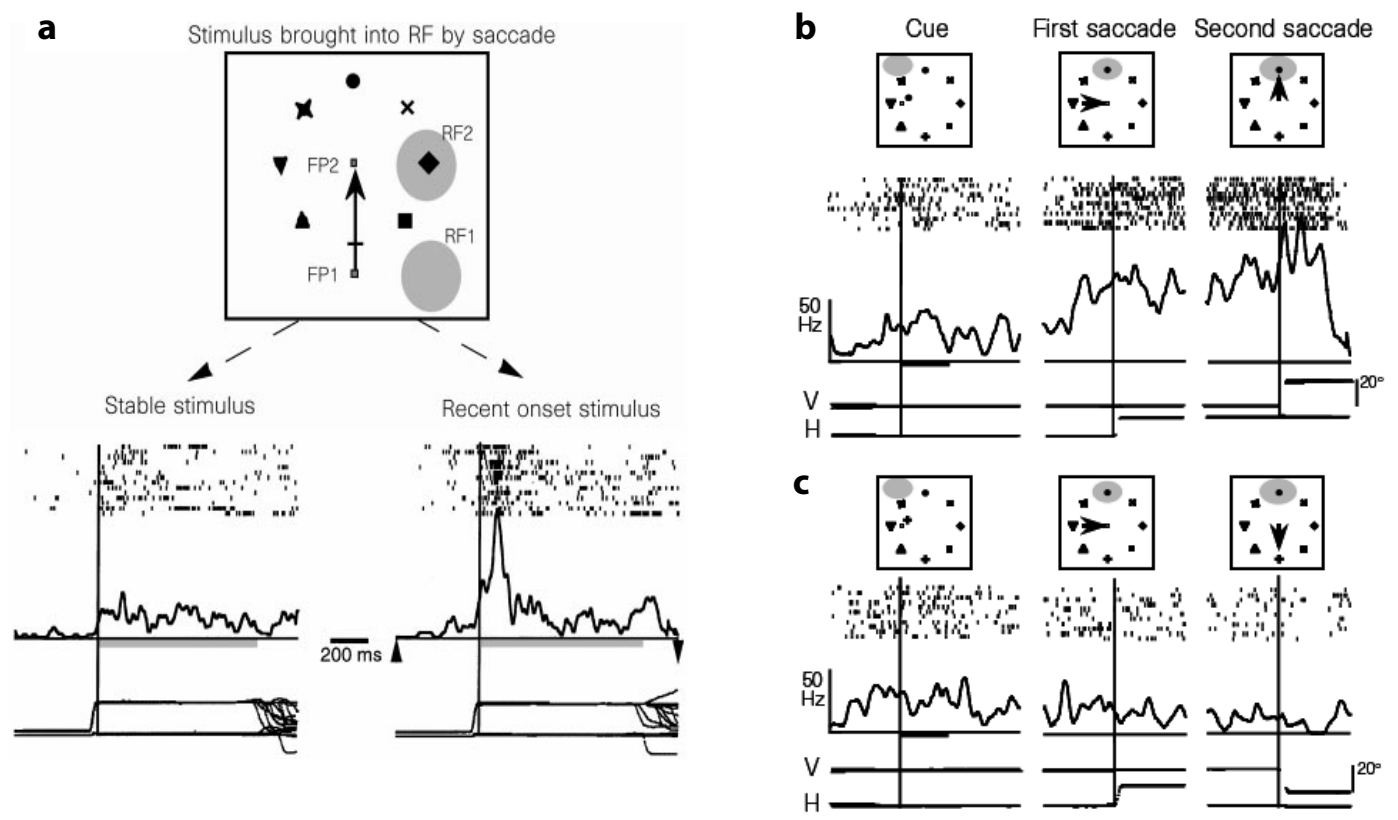

Figure 3. Example neurons respond to stable and recent onset stimulus (a), and stable saccade target (b) or distracter (c). The contents in the first row show the illustration of the visual display used by each condition in the experiment. The FP1, FP2, RF1, RF2, arrow represent the fixation point, saccade target, pre-saccadic RF, post-saccadic RF, saccade respectively. The eight components arranged in a circular are visual stimulus. Below the paradigm illustration, the raster plots and spike density histograms are shown. The time lines below the histograms show the vertical (V) and horizontal $(\mathrm{H})$ eye position. The long vertical lines indicate the saccade offset $(\mathrm{a})$, cue onset (b, c left panel), first saccade onset (b, c middle panel), and second onset (b, c right panel). (a) Neuron responses to a stable stimulus (left panel) or recent onset stimulus (right panel) that was brought into the RF by a saccade. The neuron has a stronger response when a recent onset stimulus was brought into the RF by saccade than when the stimulus was stable. (b) Neuron responses when a saccade target is brought into the neuron's RF by the first saccade while the monkeys are trained to make a two-step saccade to a cued stimulus. (c) Neuron responses when a non-saccade-target stimulus is brought into the neuron's RF. The neuron responded before the first saccade and lasted sometime after the second saccade when the RF stimulus was cued (b), but not responded when the opposite stimulus was cued (c). (Adapted from Gottlieb, Kusunoki and Goldberg, 1998)

The visual salience of a stimulus was also found to affect the remapping response in FEF (Joiner, Cavanaugh and Wurtz, 2011). In the study, when a visual stimulus which was presented in the post-saccadic RF of the recorded FEF neuron, was displayed together with distracters that were located outside of the post-saccadic RF, the remapping response was 
significantly lower than when only the stimulus in the post-saccadic RF was presented. Because the distracters decreased the visual saliency of the stimulus in the post-saccadic $R F$, the stimulus in the post-saccadic RF attracted less bottom-up attention, which decreased the remapping response (Joiner, Cavanaugh and Wurtz, 2011). Together, these results suggest that the remapping response in LIP and FEF are modulated by saliency and attention. It is possible that only salient stimuli are remapped across saccades. However, as far as we know, no study has investigated effect of top-down attention on remapping. In this thesis, we designed a paradigm to investigate how the task related top-down attention affect the remapping response in MT neurons.

In a human psychophysical remapping study, an increased TAE was reported when subjects directed attention to the adapter stimulus (Melcher, 2009). Golomb and colleagues (2008) investigated spatial attention influences both retinotopically and spatiotopocally after saccade. They used a paradigm that probed spatial attention by a spatial cue at various times after saccades. The task required subjects to discriminate the orientation (left or right) of a target tilted bar. The target bar could be at different locations depending on the spatial cue (same retinotopic location, same spatiotopic location and control location). They found facilitation at the retinotopic location of the cue (i.e. when the target was at the same retinotopic location as the cue) for about 100-200 ms after saccade when the subjects were required to maintain attention at the spatiotopic location. This facilitation of the retinotopic representation was significant immediately after the saccade, although the retinotopic location was not task-relevant in this condition. However, when the task required the subjects to keep attention at the same retinotopic location as the spatial cue, the spatiotopic representation of the cue was not facilitated, only a strong retinotopic benefit was found right after the saccade(Golomb, Chun and Mazer, 2008). The authors suggested that the native coordinate or low-level representation of spatial attention is retinotopic, and argued against the role of a spatiotopic reference frame across a saccade (also see (Golomb, Nguyen-Phuc, Mazer et al., 2010; Golomb, Pulido, Albrecht et al., 2010; Golomb, Marino, Chun et al., 2011; Golomb and Kanwisher, 2012)). 


\subsection{Attentional pointer theory}

Cavanagh and colleagues challenged the shifting receptive field and spatiotopic frame theory. They argued that remapping was the transfer of activation in the retinotopic priority map to predict the incoming stimulus due to saccades, which was similar to the finding that the neurons in the somatosensory area responded to the stimulus that was approaching (but not touching) the corresponding part of the body (MacKay and Crammond, 1987). In particular, they argued that remapping represented the updating of the 'attentional pointer' - top-down attention modulation in priority maps (e.g. SC, FEF, LIP), across saccades; then, this 'attentional pointer' updating in brain areas containing priority maps transferred to the corresponding neurons in lower visual areas (figures 4\&5). They suggested that the attended locations were the most important information for tracking the objects and maintaining visual stability, and that feature information was not remapped and could be updated after saccades. They provided several lines of evidence to support attention remapping. The first experiment used the phenomenon of apparent motion. When a dot or an object disappears in one place then appears at another location in the visual field, it seems like the dot or objects are moving from the first to the second location, even though there is no physical movement, only two dots or objects flashing at different locations. They argued that the perceptual apparent motion phenomenon was a consequence of attention being dragged from the first location of the stimulus to the second location, thereby linking the two locations together as if a single stimulus was changing locations, i.e. moving (Cavanagh, Hunt, Afraz et al., 2010a). Besides, when a saccade is made between the two stimuli (or two locations), apparent motion is seen spatiotopically rather than retinotopically (Rock and Ebenholtz, 1962), i.e. they argued that 'the attention pointer to the pre-saccadic location is currently shifted to the target's expected post-saccadic location, enabling the detection of the target displacement as apparent motion in world coordinates' (Cavanagh, Hunt, Afraz et al., 2010a, Page: 151). 

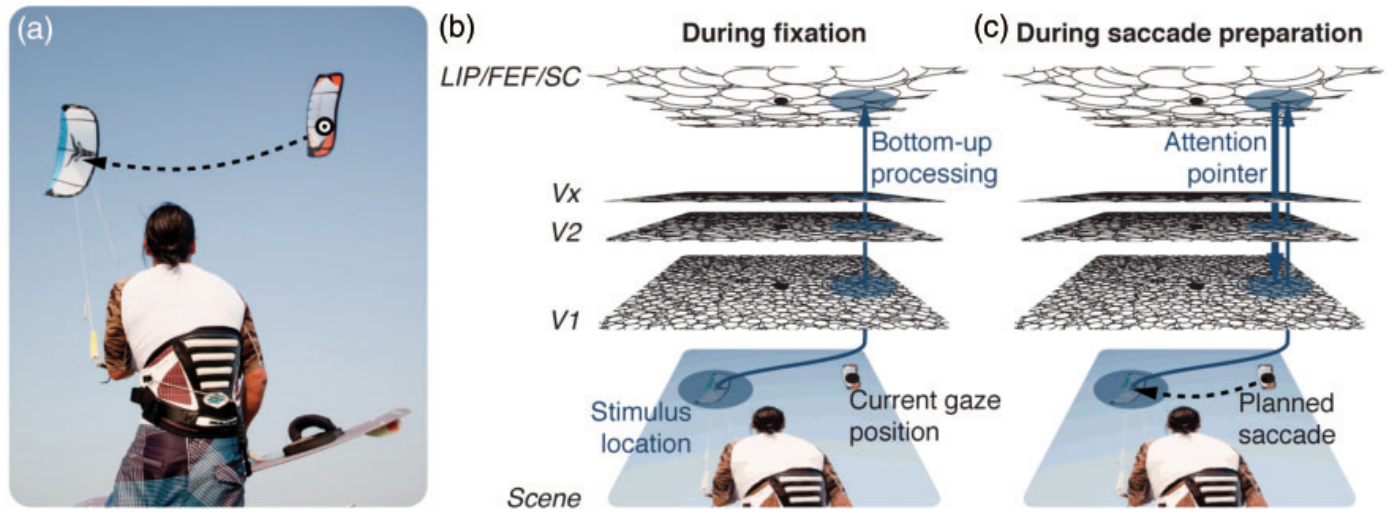

Figure 4. The illustration of attentional pointers. (a) The kite surfer is fixating on the red kite; and the blue kite will be his next saccade target. (b) The hierarchy of the visual system processing the visual scene, with each visual area retinotopically organized. The early stages (V1 to Vx) encode feature information, and late stages (such as LIP, FEF, SC) include a priority map of the current scene. (c) When a stimulus becomes more salient (here, the blue kite as the next saccade target) in the priority map, activity will increase at the corresponding location and a feedback signal will be sent to earlier visual areas. The activity in the priority maps may thus be treated as an attention pointer that indexes the corresponding locations in early visual areas (Adapted from Rolfs, 2015).

Rolfs and colleagues (2011) tested attention remapping in human subjects using a doublestep saccade paradigm. The subjects were asked to do two tasks: plan a sequence of two saccades indicated by the central cue before the actual eye movement, and discriminate and report the orientation of a target tilted grating. The discrimination task could be at the first and second saccade target locations, or the task irrelevant control location, furthermore, it also could be at the remapped location of the second saccade target, which depended on the saccade direction and amplitude. The results showed that, before saccade, in addition to a performance improvement at the first and second saccade target locations, discrimination performance was also improved at the location of the remapped location of the second saccade target, which corresponds to the retinotopic region that the second saccade target will occupy after the first saccade. Based on these results, the authors suggested that, briefly before saccades, attention was shifted to those retinal locations that the task-relevant target would occupy once the saccades had been executed, which would facilitate and speed up later eye movements before the eyes started to move. A similar performance improvement at the remapped location of a task-relevant target was found in a masking paradigm (Hunt and Cavanagh, 2011) and a covert attention experiment (Jonikaitis, Szinte, Rolfs et al., 2013; Szinte, Carrasco, Cavanagh et al., 2015). 

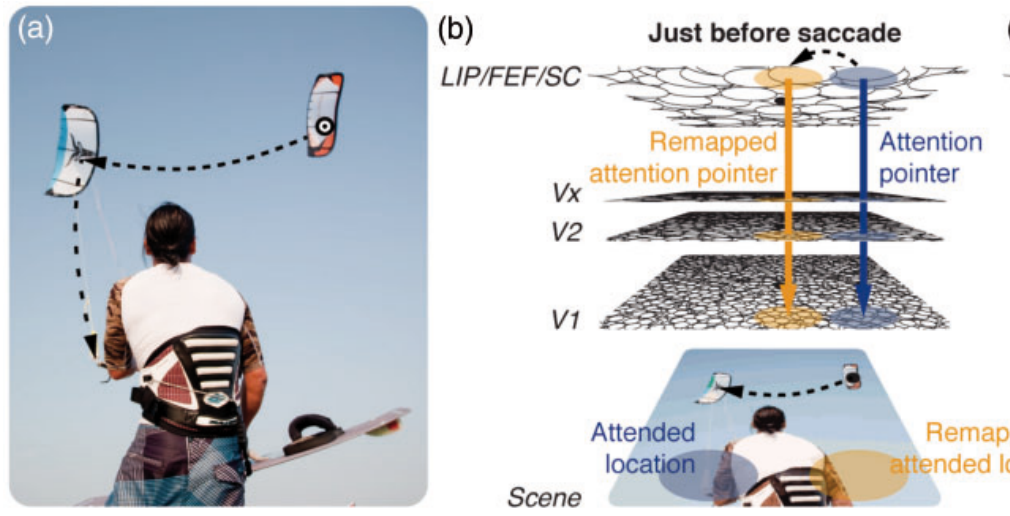

(c) Upon saccade landing

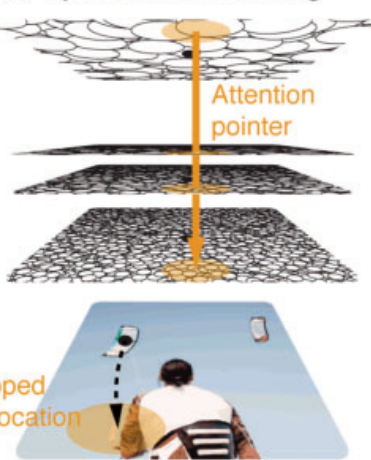

Figure 5. Attentional pointers update across saccades. (a) The kite surfer plans to make a saccade from the red kite to the blue kite and then a second saccade to the handle of his kite. (b) The handle (the second saccade target) attracts attention before the first saccade onset. The priority map is updated by increasing the activity of the corresponding neurons (yellow shade) that will process the handle after the saccades. This predictive remapping as an attention pointer then transfers to earlier visual areas (yellow arrow). (c) Right after the second saccade offset, the relevant neurons in the visual areas are modulated by attention as a consequence of this remapping (Adapted from Rolfs, 2015).

However, the physiological evidence supporting this theory is very limited, with most of the evidence based on human behavioral studies. The results of previous neuronal physiological work is difficult to interpret, as it is hard to distinguish whether the object was remapped or only attention was remapped. More neuronal physiological studies are necessary to link the behavioral and physiological evidence.

\subsection{Remapping towards saccade target}

Recently, Zirnsak and Moore (2014) provided an alternative proposal to explain remapping activity. They argued that the pre-saccadic remapping found in visual and visuomotor cortex and the perceptual changes accompanying saccades result from the selection of the saccade targets rather than an anticipation of the retinal image displacement (Zirnsak and Moore, 2014; Zirnsak, Steinmetz, Noudoost et al., 2014).

In traditional studies, the classical remapping experimental paradigms were more or less the same as the original Duhamel et al study (1992): a probe stimulus was presented either 
in a neuron's current RF or in the post-saccadic/future RF. A neuron was considered to show predictive remapping activity or a receptive field shift if the neuron responded to the probe stimulus presented in the neuron's future RF immediately before a saccade. However, previous studies never investigated the dynamics of the RF shift and other changes in RF properties, such as the size of the RF around the time of a saccade. Zirnsak and Moore (2014) used the same classical paradigm, but mapped the FEF neuron's RF long before a saccade, long after a saccade, and around the time of a saccade. This allowed a more detailed measurement of the spatial properties of the FEF neuron's RF. Nevertheless, the authors found that the FEF neurons' RFs did not remap to the post-saccadic RF/ future RF before a saccade. Instead, the FEF neurons' RFs massively converged toward the saccade target. The authors suggested that FEF neurons collectively selected the location occupied by the saccade target, rather than predicting the consequence of the saccade. The authors suggested that the neuron's RF shift to the post-saccadic RF found in previous studies was because the pre-saccadic RF of some neurons overlapped to some degree with the hypothetically remapped RF, i.e. the future RF.

To better distinguish the classical predictive remapping and convergent RF shift in human subjects, Zirnsak et al (2011) modified the TAE paradigm used by Melcher (2007) and Biber and Ilg (2011). The authors placed the adaptor away from the initial fixation point and saccade target, instead of presenting it close to the fixation point. The test was placed either close to the saccade target or at the location corresponding to the same retinotopic location of the adaptor after saccade. If the neurons' RFs were predictively remapped, the TAE should increase when the test was placed at the same retinotopic location after saccade. By contrast, if the neurons' RFs shift to or converge onto the saccade target, the TAE should be increased when the test was placed close to the saccade target compared to when the test was placed at the same retinotopic location after saccade. The authors observed the second effect. Based on these results, they argued that previous remapping results could be explained by RFs converging toward the saccade target, which was consistent with the monkey physiological data they obtained in FEF (Zirnsak, Gerhards, Kiani et al., 2011). However, the number of the subjects was very low, with only 3 subjects participating including 2 of the study authors. More experimental evidence is therefore necessary to support this idea. 
In a recent study in V4 (Neupane, Guitton and Pack, 2016)the authors found that RFs shift towards a saccade target for some neurons, but that other neurons' RFs shift towards postsaccadic RFs (or future receptive fields (FFs) in their study), and yet other neurons showed both types of remapping at different latencies. They found remapping towards the saccade target depended strongly on the relationship between saccade direction and the location of the RF. If saccades were directed towards the visual hemi-field covering the RF, remapping towards the saccade target was mainly observed. On the other hand, the other type of remapping, towards the post-saccadic RF, was independent of saccade direction.

\subsection{Eye-position gain fields}

Eye-position gain fields refer to the modulatory influence of eye-position or gaze-angle on the visual response of neurons in some brain areas. It provides a possible neuronal mechanism for creating a spatiotopic reference frame by moving the eyes while viewing the environment. Gain fields have been reported in almost all visual and visuomotor areas, as well as in some subcortical areas such as LGN and SC (Lehky, Sereno and Sereno, 2015). Gain fields code the spatiotopic location of a stimulus across a population of neurons rather than in single neurons. The spatiotopic information is obtained by the activity pattern of a group of neurons, from which the spatiotopic reference frame of the stimuli is built. The gain fields containing a self-induced image change on the retina are taken into account by the brain to build a stable representation of the visual environment. This may play a central role in the spatial constancy of visual perception. The time courses of gain fields were investigated in several brain areas (Morris, Kubischik, Hoffmann et al., 2012; Morris, Bremmer and Krekelberg, 2013,2016) and it was found that gain fields in the dorsal visual stream are accurate and fast, with some signals even capable of predicting the future eye position (Morris, Bremmer and Krekelberg, 2016). However, another study reported that the gain fields in LIP alone were too slow to localize a briefly flashed target location in space after saccade (Xu, Karachi and Goldberg, 2012). 


\section{The middle temporal area (MT)}

The middle temporal area (MT or V5) is located at posterior bank of the superior temporal sulcus (STS). It is one of the brain regions in the dorsal visual pathway and is highly involved in visual motion processing, but 'MT plays a richer and more varied role in vision' ((Born and Bradley, 2005), page: 158) than just motion perception alone. MT receives its major inputs from $V 1$ (specifically from layer $4 \mathrm{~B}$ ), but also receives feed-forward inputs from the lateral geniculate nucleus (LGN), V2, V3, V3A, VP and PIP; besides, it receives feedback/modulatory inputs from higher level cortex such as FEF and LIP (Maunsell and van Essen, 1983c; Felleman and Van Essen, 1991; Sincich, Park, Wohlgemuth et al., 2004). One of its major projection regions is the medial superior temporal area (MST). MT is a retinotopically organized region region, and every visual neuron in this area has a certain receptive field (RF) occupying a part of the contralateral visual hemi-field when the eyes keep fixation on a central point. Each MT area in the two hemispheres contains an approximately intact map of the contralateral visual hemi-field (Born and Bradley, 2005). About half of the MT neurons' RFs are in the central 15 degrees of the visual field (Van Essen, Maunsell and Bixby, 1981). Visual responses of MT neurons have five basic tuning properties (Maunsell and Van Essen, 1983b, a; Born and Bradley, 2005): 1) retinal position (due to the specific RF); 2) stimulus size (because of surround suppression effects); 3) binocular disparity; 4) motion direction and 5) motion speed of the stimuli in the RF. In terms of remapping, a previous of study has shown that the MT neurons do not show predictive remapping(Ong and Bisley, 2011); therefore, in this thesis, we will focus on the other type of remapping, the post-saccadic memory trace.

Based on these properties of MT neurons, and especially the retinotopic organization and appropriately small size of the RF, motion direction tuning, as well as the effect of topdown attention modulation on MT neurons (Treue, 2001, 2003; Busse, Katzner and Treue, 2008; Daliri, Kozyrev and Treue, 2016), we decided to record from MT. The retinotopic organization makes it an ideal area to investigate the shift of attention modulation and remapping across a saccade; and the appropriate size of MT neuronal RFs makes it possible to clearly delineate regions outside the RF. Moreover, the monkeys do not have to make big saccades. Therefor, MT is an ideal area to investigate the neuronal dynamics of attention shifts across saccades and trans-saccadic remapping properties such as feature tuning in the remapped response. In this thesis, we focused on these two issues by 
recording from MT neurons when the monkeys were trained to perform a saccade along with an attention task.

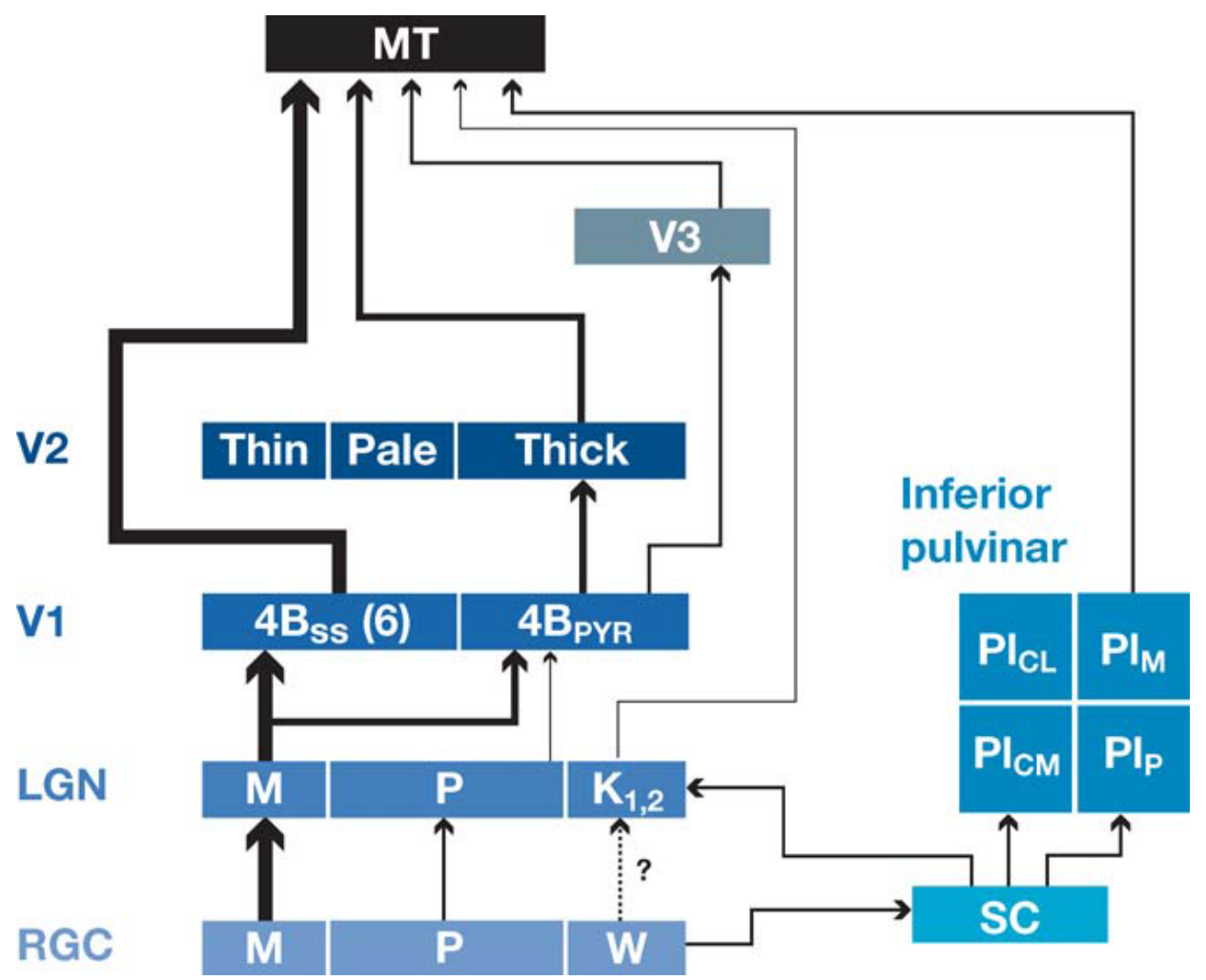

Figure 6. A map of the major projections into MT. Line thickness roughly represents the strength of the projection. The thickest lines indicate that the major direct input into MT is from V1. Abbreviations: 4Bss, spiny stellate neurons in layer 4B; 4B 4 PYR, pyramidal neurons in layer 4B; LGN, lateral geniculate nucleus; $\mathrm{M}$, magnocellular stream; $\mathrm{P}$, parvocellular stream; $\mathrm{K}$, koniocellular layers of LGN; $\mathrm{PI}_{\mathrm{CL}}$, central lateral nucleus of the inferior pulvinar; $\mathrm{PI}_{\mathrm{CM}}$, central medial nucleus of the inferior pulvinar; $\mathrm{PI}_{\mathrm{M}}$, medial nucleus of the inferior pulvinar; $\mathrm{PI}_{\mathrm{P}}$, posterior nucleus of the inferior pulvinar; RGC, retinal ganglion cells; SC, superior colliculus; VP, ventral posterior area. (Adapted from Born and Bradley, 2005)

\section{General summary}

In this thesis, at first, we studied a very critical and natural phenomenon, namely, maintaining attention on a spatially stable target across saccades. We recorded from wellisolated neurons in MT while the monkeys performed an attentional task. We investigated 
the dynamics of how attention stopped modulating a neuron's response when a saccade brought a target out of its RF in Experiment one, and we also investigated the dynamics of how attention starts to modulate a neuron's response when a saccade brought a target into its RF in Experiment two. We also investigated human performance around the time of saccade as well; we focused on the dynamics of performance recovery after the saccade.

In the second part of this thesis, we investigate some important properties of remapping. Remapping has been reported and researched intensively in the last decades in both nonhuman primates and human subjects. However, there are still many debates on this topic, such as whether visual features and unattended objects are remapped across saccades. In this thesis, we designed and performed several experiments to investigate the perceptual and neural properties around the time of saccades to better understand remapping across saccades. At first, we will ask whether a remapped response can be found in MT. If the answer is yes, more importantly, we will investigate the modulation of top-down attention on the remapping response, and at the same time, we will investigate whether visual features are remapped across a saccade. Our study will provide important evidence to illuminate the debates on this topic, and contribute new insights into understanding what remapping is per se. 


\section{References}

Afraz SR, Cavanagh P (2008) Retinotopy of the face aftereffect. Vision Res 48:42-54.

Anton-Erxleben K, Stephan VM, Treue S (2009) Attention reshapes center-surround receptive field structure in macaque cortical area MT. Cereb Cortex 19:2466-2478.

Bahill AT, Bahill KA, Clark MR, Stark L (1975) Closely spaced saccades. Invest Ophthalmol 14:317321.

Barash S, Bracewell RM, Fogassi L, Gnadt JW, Andersen RA (1991) Saccade-related activity in the lateral intraparietal area. II. Spatial properties. J Neurophysiol 66:1109-1124.

Bellebaum C, Daum I (2006) Time course of cross-hemispheric spatial updating in the human parietal cortex. Behav Brain Res 169:150-161.

Bellebaum C, Hoffmann KP, Daum I (2005) Post-saccadic updating of visual space in the posterior parietal cortex in humans. Behav Brain Res 163:194-203.

Bellebaum C, Daum I, Koch B, Schwarz M, Hoffmann KP (2005) The role of the human thalamus in processing corollary discharge. Brain 128:1139-1154.

Berman RA, Wurtz RH (2011) Signals conveyed in the pulvinar pathway from superior colliculus to cortical area MT. J Neurosci 31:373-384.

Biber U, Ilg UJ (2011) Visual stability and the motion aftereffect: a psychophysical study revealing spatial updating. PLoS One 6:e16265.

Born RT, Bradley DC (2005) Structure and function of visual area MT. Annu Rev Neurosci 28:157189.

Boynton GM (2005) Attention and visual perception. Curr Opin Neurobiol 15:465-469.

Bridgeman B, Hendry D, Stark L (1975) Failure to detect displacement of the visual world during saccadic eye movements. Vision Res 15:719-722.

Brown JW, Hanes DP, Schall JD, Stuphorn V (2008) Relation of frontal eye field activity to saccade initiation during a countermanding task. Exp Brain Res 190:135-151.

Buffalo EA, Fries P, Landman R, Liang H, Desimone R (2010) A backward progression of attentional effects in the ventral stream. Proc Natl Acad Sci U S A 107:361-365.

Burr DC, Morrone MC (2011) Spatiotopic coding and remapping in humans. Philos Trans R Soc Lond B Biol Sci 366:504-515.

Burr DC, Morrone MC, Ross J (1994) Selective suppression of the magnocellular visual pathway during saccadic eye movements. Nature 371:511-513.

Burr DC, Holt J, Johnstone JR, Ross J (1982) Selective depression of motion sensitivity during saccades. J Physiol 333:1-15.

Buschman TJ, Miller EK (2007) Top-down versus bottom-up control of attention in the prefrontal and posterior parietal cortices. Science 315:1860-1862.

Busse L, Katzner S, Treue S (2008) Temporal dynamics of neuronal modulation during exogenous and endogenous shifts of visual attention in macaque area MT. Proc Natl Acad Sci U S A 105:16380-16385.

Carrasco M, Ling S, Read S (2004) Attention alters appearance. Nat Neurosci 7:308-313.

Castet E, Masson GS (2000) Motion perception during saccadic eye movements. Nat Neurosci 3:177183.

Castet E, Jeanjean S, Masson GS (2002) Motion perception of saccade-induced retinal translation. Proc Natl Acad Sci U S A 99:15159-15163.

Castiello U, Umilta C (1990) Size of the attentional focus and efficiency of processing. Acta Psychol (Amst) 73:195-209.

Cavanagh P, Hunt AR, Afraz A, Rolfs M (2010a) Visual stability based on remapping of attention pointers. Trends Cogn Sci 14:147-153.

Cavanagh P, Hunt AR, Afraz A, Rolfs M (2010c) Attention Pointers: Response to Mayo and Sommer. Trends Cogn Sci 14:390-391.

Cavanaugh J, Wurtz RH (2004) Subcortical modulation of attention counters change blindness. J Neurosci 24:11236-11243.

Cavanaugh J, Berman RA, Joiner WM, Wurtz RH (2016) Saccadic Corollary Discharge Underlies Stable Visual Perception. J Neurosci 36:31-42. 
Chambers CD, Mattingley JB (2005) Neurodisruption of selective attention: insights and implications. Trends Cogn Sci 9:542-550.

Churan J, Guitton D, Pack CC (2012) Perisaccadic remapping and rescaling of visual responses in macaque superior colliculus. PLoS One 7:e52195.

Cohen MR, Maunsell JH (2009) Attention improves performance primarily by reducing interneuronal correlations. Nat Neurosci 12:1594-1600.

Colby CL, Duhamel JR, Goldberg ME (1996) Visual, presaccadic, and cognitive activation of single neurons in monkey lateral intraparietal area. J Neurophysiol 76:2841-2852.

Corbetta M (1998) Frontoparietal cortical networks for directing attention and the eye to visual locations: identical, independent, or overlapping neural systems? Proc Natl Acad Sci U S A 95:831-838.

Corbetta M, Akbudak E, Conturo TE, Snyder AZ, Ollinger JM, Drury HA, Linenweber MR, Petersen SE, Raichle ME, Van Essen DC, Shulman GL (1998) A common network of functional areas for attention and eye movements. Neuron 21:761-773.

d'Avossa G, Tosetti M, Crespi S, Biagi L, Burr DC, Morrone MC (2007) Spatiotopic selectivity of BOLD responses to visual motion in human area MT. Nat Neurosci 10:249-255.

Daliri MR, Kozyrev V, Treue S (2016) Attention enhances stimulus representations in macaque visual cortex without affecting their signal-to-noise level. Sci Rep 6:27666.

Deubel H (2008) The time course of presaccadic attention shifts. Psychol Res 72:630-640.

Deubel H, Schneider WX (1996) Saccade target selection and object recognition: evidence for a common attentional mechanism. Vision Res 36:1827-1837.

Deubel H, Elsner T, Hauske G (1987) Saccadic eye movements and the detection of fast-moving gratings. Biol Cybern 57:37-45.

Diamond MR, Ross J, Morrone MC (2000) Extraretinal control of saccadic suppression. J Neurosci 20:3449-3455

Dickinson JE, Mighall HK, Almeida RA, Bell J, Badcock DR (2012) Rapidly acquired shape and face aftereffects are retinotopic and local in origin. Vision Res 65:1-11.

Dorr M, Bex PJ (2013) Peri-saccadic natural vision. J Neurosci 33:1211-1217.

Duhamel JR, Colby CL, Goldberg ME (1992) The updating of the representation of visual space in parietal cortex by intended eye movements. Science 255:90-92.

Dunn CA, Hall NJ, Colby CL (2010) Spatial updating in monkey superior colliculus in the absence of the forebrain commissures: dissociation between superficial and intermediate layers. J Neurophysiol 104:1267-1285.

Eriksen CW, Webb JM, Fournier LR (1990) How much processing do nonattended stimuli receive? Apparently very little, but. Percept Psychophys 47:477-488.

Ezzati A, Golzar A, Afraz AS (2008) Topography of the motion aftereffect with and without eye movements. J Vis 8:23 21-16.

Felleman DJ, Van Essen DC (1991) Distributed hierarchical processing in the primate cerebral cortex. Cereb Cortex 1:1-47.

Fries P, Womelsdorf T, Oostenveld R, Desimone R (2008) The effects of visual stimulation and selective visual attention on rhythmic neuronal synchronization in macaque area V4. J Neurosci 28:4823-4835.

Friesen CK, Kingstone A (2003) Abrupt onsets and gaze direction cues trigger independent reflexive attentional effects. Cognition 87:B1-10.

Gaymard B (2012) Cortical and sub-cortical control of saccades and clinical application. Rev Neurol (Paris) 168:734-740.

Goldberg ME, Bruce CJ (1990) Primate frontal eye fields. III. Maintenance of a spatially accurate saccade signal. J Neurophysiol 64:489-508.

Goldberg ME, Bisley JW, Powell KD, Gottlieb J (2006) Saccades, salience and attention: the role of the lateral intraparietal area in visual behavior. Prog Brain Res 155:157-175.

Golomb JD, Kanwisher N (2012) Retinotopic memory is more precise than spatiotopic memory. Proc Natl Acad Sci U S A 109:1796-1801.

Golomb JD, Chun MM, Mazer JA (2008) The native coordinate system of spatial attention is retinotopic. J Neurosci 28:10654-10662. 
Golomb JD, Marino AC, Chun MM, Mazer JA (2011) Attention doesn't slide: spatiotopic updating after eye movements instantiates a new, discrete attentional locus. Atten Percept Psychophys 73:7-14.

Golomb JD, Nguyen-Phuc AY, Mazer JA, McCarthy G, Chun MM (2010) Attentional facilitation throughout human visual cortex lingers in retinotopic coordinates after eye movements. J Neurosci 30:10493-10506.

Golomb JD, Pulido VZ, Albrecht AR, Chun MM, Mazer JA (2010) Robustness of the retinotopic attentional trace after eye movements. J Vis 10:19 11-12.

Gottlieb J (2007) From a different point of view: extrastriate cortex integrates information across saccades. Focus on "Remapping in human visual cortex". J Neurophysiol 97:961-962.

Gottlieb JP, Kusunoki M, Goldberg ME (1998) The representation of visual salience in monkey parietal cortex. Nature 391:481-484.

Gregoriou GG, Gotts SJ, Zhou H, Desimone R (2009) High-frequency, long-range coupling between prefrontal and visual cortex during attention. Science 324:1207-1210.

Grosbras MH, Paus T (2002) Transcranial magnetic stimulation of the human frontal eye field: effects on visual perception and attention. J Cogn Neurosci 14:1109-1120.

Hall NJ, Colby CL (2011) Remapping for visual stability. Philos Trans R Soc Lond B Biol Sci 366:528539.

Hass CA, Horwitz GD (2011) Effects of microsaccades on contrast detection and V1 responses in macaques. J Vis 11:1-17.

Heiser LM, Colby CL (2006) Spatial updating in area LIP is independent of saccade direction. J Neurophysiol 95:2751-2767.

Herrington TM, Assad JA (2009) Neural activity in the middle temporal area and lateral intraparietal area during endogenously cued shifts of attention. J Neurosci 29:14160-14176.

Herrington TM, Assad JA (2010) Temporal sequence of attentional modulation in the lateral intraparietal area and middle temporal area during rapid covert shifts of attention. J Neurosci 30:3287-3296.

Higgins E, Rayner K (2015) Transsaccadic processing: stability, integration, and the potential role of remapping. Atten Percept Psychophys 77:3-27.

Hoffman JE, Subramaniam B (1995) The role of visual attention in saccadic eye movements. Percept Psychophys 57:787-795.

Hunt AR, Cavanagh P (2011) Remapped visual masking. J Vis 11:13.

Ibbotson MR, Price NS, Crowder NA, Ono S, Mustari MJ (2007) Enhanced motion sensitivity follows saccadic suppression in the superior temporal sulcus of the macaque cortex. Cereb Cortex 17:1129-1138.

Ignashchenkova A, Dicke PW, Haarmeier T, Thier P (2004) Neuron-specific contribution of the superior colliculus to overt and covert shifts of attention. Nat Neurosci 7:56-64.

Inaba N, Kawano K (2014) Neurons in cortical area MST remap the memory trace of visual motion across saccadic eye movements. Proc Natl Acad Sci U S A 111:7825-7830.

James W (1890) The Principles of Psychology: H. Holt.

Joiner WM, Cavanaugh J, Wurtz RH (2011) Modulation of shifting receptive field activity in frontal eye field by visual salience. J Neurophysiol 106:1179-1190.

Jonikaitis D, Szinte M, Rolfs M, Cavanagh P (2013) Allocation of attention across saccades. J Neurophysiol 109:1425-1434.

Judge SJ, Wurtz RH, Richmond BJ (1980) Vision during saccadic eye movements. I. Visual interactions in striate cortex. J Neurophysiol 43:1133-1155.

Kagan I, Gur M, Snodderly DM (2008) Saccades and drifts differentially modulate neuronal activity in V1: effects of retinal image motion, position, and extraretinal influences. J Vis 8:19 11-25.

Khayat PS, Spekreijse H, Roelfsema PR (2004) Correlates of transsaccadic integration in the primary visual cortex of the monkey. Proc Natl Acad Sci U S A 101:12712-12717.

Knapen T, Rolfs M, Cavanagh P (2009) The reference frame of the motion aftereffect is retinotopic. J Vis 9:16 11-17.

Kowler E (2011) Eye movements: the past 25 years. Vision Res 51:1457-1483.

Kowler E, Anderson E, Dosher B, Blaser E (1995) The role of attention in the programming of saccades. Vision Res 35:1897-1916. 
Kusunoki M, Goldberg ME (2003) The time course of perisaccadic receptive field shifts in the lateral intraparietal area of the monkey. J Neurophysiol 89:1519-1527.

Lehky SR, Sereno ME, Sereno AB (2015) Characteristics of Eye-Position Gain Field Populations Determine Geometry of Visual Space. Front Integr Neurosci 9:72.

Luck SJ, Ford MA (1998) On the role of selective attention in visual perception. Proc Natl Acad Sci U S A 95:825-830.

Luck SJ, Chelazzi L, Hillyard SA, Desimone R (1997) Neural mechanisms of spatial selective attention in areas V1, V2, and V4 of macaque visual cortex. J Neurophysiol 77:24-42.

MacKay WA, Crammond DJ (1987) Neuronal correlates in posterior parietal lobe of the expectation of events. Behav Brain Res 24:167-179.

Marino AC, Mazer JA (2016) Perisaccadic Updating of Visual Representations and Attentional States: Linking Behavior and Neurophysiology. Front Syst Neurosci 10:3.

Mathot S, Theeuwes J (2010) Evidence for the predictive remapping of visual attention. Exp Brain Res 200:117-122.

Mathot S, Theeuwes J (2011) Visual attention and stability. Philos Trans R Soc Lond B Biol Sci 366:516-527.

Mathot S, Theeuwes J (2013) A reinvestigation of the reference frame of the tilt-adaptation aftereffect. Sci Rep 3:1152.

Matthews PB (1982) Where does Sherrington's "muscular sense" originate? Muscles, joints, corollary discharges? Annu Rev Neurosci 5:189-218.

Maunsell JH, Van Essen DC (1983a) Functional properties of neurons in middle temporal visual area of the macaque monkey. I. Selectivity for stimulus direction, speed, and orientation. J Neurophysiol 49:1127-1147.

Maunsell JH, Van Essen DC (1983b) Functional properties of neurons in middle temporal visual area of the macaque monkey. II. Binocular interactions and sensitivity to binocular disparity. J Neurophysiol 49:1148-1167.

Maunsell JH, van Essen DC (1983c) The connections of the middle temporal visual area (MT) and their relationship to a cortical hierarchy in the macaque monkey. J Neurosci 3:2563-2586.

Mayer AR, Dorflinger JM, Rao SM, Seidenberg M (2004) Neural networks underlying endogenous and exogenous visual-spatial orienting. Neuroimage 23:534-541.

Medendorp WP, Goltz HC, Vilis T (2006) Directional selectivity of BOLD activity in human posterior parietal cortex for memory-guided double-step saccades. J Neurophysiol 95:1645-1655.

Medendorp WP, Goltz HC, Vilis T, Crawford JD (2003) Gaze-centered updating of visual space in human parietal cortex. J Neurosci 23:6209-6214.

Melcher D (2005) Spatiotopic transfer of visual-form adaptation across saccadic eye movements. Curr Biol 15:1745-1748.

Melcher D (2007) Predictive remapping of visual features precedes saccadic eye movements. Nat Neurosci 10:903-907.

Melcher D (2009) Selective attention and the active remapping of object features in trans-saccadic perception. Vision Res 49:1249-1255.

Melcher D (2011) Visual stability. Philos Trans R Soc Lond B Biol Sci 366:468-475.

Merriam EP, Genovese CR, Colby CL (2003) Spatial updating in human parietal cortex. Neuron 39:361-373.

Merriam EP, Genovese CR, Colby CL (2007) Remapping in human visual cortex. J Neurophysiol 97:1738-1755.

Mirpour K, Bisley JW (2012) Anticipatory remapping of attentional priority across the entire visual field. J Neurosci 32:16449-16457.

Mitchell JF, Sundberg KA, Reynolds JH (2007) Differential attention-dependent response modulation across cell classes in macaque visual area V4. Neuron 55:131-141.

Moore T, Armstrong KM (2003) Selective gating of visual signals by microstimulation of frontal cortex. Nature 421:370-373.

Moore T, Fallah M (2004) Microstimulation of the frontal eye field and its effects on covert spatial attention. J Neurophysiol 91:152-162.

Moore T, Tolias AS, Schiller PH (1998) Visual representations during saccadic eye movements. Proc Natl Acad Sci U S A 95:8981-8984. 
Moran J, Desimone R (1985) Selective attention gates visual processing in the extrastriate cortex. Science 229:782-784.

Morgan EJ, Ball K, Smith DT (2014) The role of the oculomotor system in covert social attention. Atten Percept Psychophys 76:1265-1270.

Morris AP, Bremmer F, Krekelberg B (2013) Eye-position signals in the dorsal visual system are accurate and precise on short timescales. J Neurosci 33:12395-12406.

Morris AP, Bremmer F, Krekelberg B (2016) The Dorsal Visual System Predicts Future and Remembers Past Eye Position. Front Syst Neurosci 10:9.

Morris AP, Kubischik M, Hoffmann KP, Krekelberg B, Bremmer F (2012) Dynamics of eye-position signals in the dorsal visual system. Curr Biol 22:173-179.

Morris AP, Liu CC, Cropper SJ, Forte JD, Krekelberg B, Mattingley JB (2010) Summation of visual motion across eye movements reflects a nonspatial decision mechanism. J Neurosci 30:9821-9830.

Muller JR, Philiastides MG, Newsome WT (2005) Microstimulation of the superior colliculus focuses attention without moving the eyes. Proc Natl Acad Sci U S A 102:524-529.

Nakamura K, Colby CL (2002) Updating of the visual representation in monkey striate and extrastriate cortex during saccades. Proc Natl Acad Sci U S A 99:4026-4031.

Neupane S, Guitton D, Pack CC (2016) Two distinct types of remapping in primate cortical area V4. Nat Commun 7:10402.

Noudoost B, Chang MH, Steinmetz NA, Moore T (2010) Top-down control of visual attention. Curr Opin Neurobiol 20:183-190.

Ong WS, Bisley JW (2011) A lack of anticipatory remapping of retinotopic receptive fields in the middle temporal area. J Neurosci 31:10432-10436.

Parks NA, Corballis PM (2008) Electrophysiological correlates of presaccadic remapping in humans. Psychophysiology 45:776-783.

Parks NA, Corballis PM (2010) Human transsaccadic visual processing: presaccadic remapping and postsaccadic updating. Neuropsychologia 48:3451-3458.

Peterburs J, Gajda K, Hoffmann KP, Daum I, Bellebaum C (2011) Electrophysiological correlates of inter- and intrahemispheric saccade-related updating of visual space. Behav Brain Res 216:496-504

Petersen SE, Robinson DL, Morris JD (1987) Contributions of the pulvinar to visual spatial attention. Neuropsychologia 25:97-105.

Pierrot-Deseilligny C, Rivaud S, Gaymard B, Muri R, Vermersch AI (1995) Cortical control of saccades. Ann Neurol 37:557-567.

Posner MI (1980) Orienting of attention. Q J Exp Psychol 32:3-25.

Rao V, DeAngelis GC, Snyder LH (2012) Neural correlates of prior expectations of motion in the lateral intraparietal and middle temporal areas. J Neurosci 32:10063-10074.

Reppas JB, Usrey WM, Reid RC (2002) Saccadic eye movements modulate visual responses in the lateral geniculate nucleus. Neuron 35:961-974.

Rizzolatti G, Riggio L, Dascola I, Umilta C (1987) Reorienting attention across the horizontal and vertical meridians: evidence in favor of a premotor theory of attention. Neuropsychologia 25:31-40.

Robinson DL, Wurtz RH (1976) Use of an extraretinal signal by monkey superior colliculus neurons to distinguish real from self-induced stimulus movement. J Neurophysiol 39:852-870.

Rock I, Ebenholtz S (1962) Stroboscopic movement based on change of phenomenal rather than retinal location. Am J Psychol 75:193-207.

Rolfs M (2015) Attention in Active Vision: A Perspective on Perceptual Continuity Across Saccades. Perception 44:900-919.

Ross J, Morrone MC, Goldberg ME, Burr DC (2001) Changes in visual perception at the time of saccades. Trends Neurosci 24:113-121.

Saalmann YB, Pigarev IN, Vidyasagar TR (2007) Neural mechanisms of visual attention: how topdown feedback highlights relevant locations. Science 316:1612-1615.

Sincich LC, Park KF, Wohlgemuth MJ, Horton JC (2004) Bypassing V1: a direct geniculate input to area MT. Nat Neurosci 7:1123-1128. 
Snodderly DM (1987) Effects of light and dark environments on macaque and human fixational eye movements. Vision Res 27:401-415.

Sommer MA, Wurtz RH (2002) A pathway in primate brain for internal monitoring of movements. Science 296:1480-1482.

Sommer MA, Wurtz RH (2004) What the brain stem tells the frontal cortex. II. Role of the SC-MDFEF pathway in corollary discharge. J Neurophysiol 91:1403-1423.

Sommer MA, Wurtz RH (2006) Influence of the thalamus on spatial visual processing in frontal cortex. Nature 444:374-377.

Sommer MA, Wurtz RH (2008) Brain circuits for the internal monitoring of movements. Annu Rev Neurosci 31:317-338.

Sperry RW (1950) Neural basis of the spontaneous optokinetic response produced by visual inversion. J Comp Physiol Psychol 43:482-489.

Steinmetz NA, Moore T (2014) Eye movement preparation modulates neuronal responses in area V4 when dissociated from attentional demands. Neuron 83:496-506.

Sylvester R, Rees G (2006) Extraretinal saccadic signals in human LGN and early retinotopic cortex. Neuroimage 30:214-219.

Szinte M, Carrasco M, Cavanagh P, Rolfs M (2015) Attentional trade-offs maintain the tracking of moving objects across saccades. J Neurophysiol 113:2220-2231.

Thiele A, Henning P, Kubischik M, Hoffmann KP (2002) Neural mechanisms of saccadic suppression. Science 295:2460-2462.

Thompson KG, Biscoe KL, Sato TR (2005) Neuronal basis of covert spatial attention in the frontal eye field. J Neurosci 25:9479-9487.

Tolias AS, Moore T, Smirnakis SM, Tehovnik EJ, Siapas AG, Schiller PH (2001) Eye movements modulate visual receptive fields of V4 neurons. Neuron 29:757-767.

Tootell RB, Hadjikhani N, Hall EK, Marrett S, Vanduffel W, Vaughan JT, Dale AM (1998) The retinotopy of visual spatial attention. Neuron 21:1409-1422.

Treue S (2001) Neural correlates of attention in primate visual cortex. Trends Neurosci 24:295-300.

Treue S (2003) Visual attention: the where, what, how and why of saliency. Curr Opin Neurobiol 13:428-432.

Treue S, Maunsell JH (1996) Attentional modulation of visual motion processing in cortical areas MT and MST. Nature 382:539-541.

Treue S, Martinez Trujillo JC (1999) Feature-based attention influences motion processing gain in macaque visual cortex. Nature 399:575-579.

Turi M, Burr D (2012) Spatiotopic perceptual maps in humans: evidence from motion adaptation. Proc Biol Sci 279:3091-3097.

Umeno MM, Goldberg ME (1997) Spatial processing in the monkey frontal eye field. I. Predictive visual responses. J Neurophysiol 78:1373-1383.

Umeno MM, Goldberg ME (2001) Spatial processing in the monkey frontal eye field. II. Memory responses. J Neurophysiol 86:2344-2352.

Van Der Werf J, Jensen 0, Fries P, Medendorp WP (2008) Gamma-band activity in human posterior parietal cortex encodes the motor goal during delayed prosaccades and antisaccades. J Neurosci 28:8397-8405.

Van Essen DC, Maunsell JH, Bixby JL (1981) The middle temporal visual area in the macaque: myeloarchitecture, connections, functional properties and topographic organization. J Comp Neurol 199:293-326.

Volkmann FC (1986) Human visual suppression. Vision Res 26:1401-1416.

Walker MF, Fitzgibbon EJ, Goldberg ME (1995) Neurons in the monkey superior colliculus predict the visual result of impending saccadic eye movements. J Neurophysiol 73:1988-2003.

Wang X, Fung CC, Guan S, Wu S, Goldberg ME, Zhang M (2016) Perisaccadic Receptive Field Expansion in the Lateral Intraparietal Area. Neuron.

Wardak C, Olivier E, Duhamel JR (2002) Saccadic target selection deficits after lateral intraparietal area inactivation in monkeys. J Neurosci 22:9877-9884.

Wardak C, Olivier E, Duhamel JR (2004) A deficit in covert attention after parietal cortex inactivation in the monkey. Neuron 42:501-508. 
Wardak C, Olivier E, Duhamel JR (2011) The relationship between spatial attention and saccades in the frontoparietal network of the monkey. Eur J Neurosci 33:1973-1981.

Wenderoth P, Wiese M (2008) Retinotopic encoding of the direction aftereffect. Vision Res 48:19491954.

Wolfe BA, Whitney D (2015) Saccadic remapping of object-selective information. Atten Percept Psychophys 77:2260-2269.

Womelsdorf T, Anton-Erxleben K, Pieper F, Treue S (2006) Dynamic shifts of visual receptive fields in cortical area MT by spatial attention. Nat Neurosci 9:1156-1160.

Wurtz RH (2008) Neuronal mechanisms of visual stability. Vision Res 48:2070-2089.

Wurtz RH, Joiner WM, Berman RA (2011) Neuronal mechanisms for visual stability: progress and problems. Philos Trans R Soc Lond B Biol Sci 366:492-503.

Wurtz RH, McAlonan K, Cavanaugh J, Berman RA (2011) Thalamic pathways for active vision. Trends Cogn Sci 15:177-184.

Xu BY, Karachi C, Goldberg ME (2012) The postsaccadic unreliability of gain fields renders it unlikely that the motor system can use them to calculate target position in space. Neuron 76:1201-1209.

Yoshimoto S, Uchida-Ota M, Takeuchi T (2014) The reference frame of visual motion priming depends on underlying motion mechanisms. J Vis 14.

Zanos TP, Mineault PJ, Guitton D, Pack CC (2016) Mechanisms of Saccadic Suppression in Primate Cortical Area V4. J Neurosci 36:9227-9239.

Zimmermann E, Morrone MC, Fink GR, Burr D (2013) Spatiotopic neural representations develop slowly across saccades. Curr Biol 23:R193-194.

Zirnsak M, Moore T (2014) Saccades and shifting receptive fields: anticipating consequences or selecting targets? Trends Cogn Sci 18:621-628.

Zirnsak M, Gerhards RG, Kiani R, Lappe M, Hamker FH (2011) Anticipatory saccade target processing and the presaccadic transfer of visual features. J Neurosci 31:17887-17891.

Zirnsak M, Steinmetz NA, Noudoost B, Xu KZ, Moore T (2014) Visual space is compressed in prefrontal cortex before eye movements. Nature 507:504-507.

Zuber BL, Stark L (1966) Saccadic suppression: elevation of visual threshold associated with saccadic eye movements. Exp Neurol 16:65-79. 


\section{Abbreviations}

BOLD: blood-oxygenation level dependent

CD: corollary discharge

EEG: electroencephalography

FEF: frontal eye field

fMRI: functional magnetic resonance imaging

LFP: local field potential

LGN: lateral geniculate nucleus

LIP: lateral intraparietal area

MAE: motion after effect

MD: medial dorsal

MEG: magnetoencephalography

MST: medial superior temporal

MT: middle temporal

RDP: random dot pattern

RF: receptive field

SC: superior colliculus

STS: superior temporal sulcus

TAE: tilt after effect

TMS: transcranial magnetic stimulation 
Chapter 2

Attentional remapping in macaque area MT is well-synchronized to saccades 


\section{Attentional remapping in macaque area MT is well- synchronized to saccades}

Tao Yao ${ }^{1}$, Stefan Treue ${ }^{1,2,3}$, B. Suresh Krishna ${ }^{1 *}$

Affiliations:

1 Cognitive Neuroscience Laboratory, German Primate Center, 37077 Goettingen, Germany.

2 Bernstein Center for Computational Neuroscience, 37077 Goettingen, Germany.

3 Faculty of Biology and Psychology, Goettingen University, 37073

Goettingen, Germany.

Correspondence: B. Suresh Krishna, skrishna@dpz.eu

*Lead Contact 


\section{Summary}

2

3 Humans and monkeys are able to keep track of relevant visual stimuli while

4 making scanning saccadic eye-movements. Maintaining top-down attention

5 on a relevant stimulus across saccades necessitates a rapid, saccade-

6 synchronized remapping of attentional modulation from the target population

7 representing the stimulus before the saccade to the one representing it after

8 the saccade. Currently, the time-course of remapping is unknown. We

9 trained two monkeys to make a saccade while maintaining top-down

10 attention at a fixed spatial location. Recording from visual area MT, we find

11 that attentional remapping is temporally well-sychronized to the saccade.

12 Attentional modulation crosses over from the pre-saccadic to the post-

13 saccadic target population at 31 and $52 \mathrm{~ms}$ after saccade offset in the two

14 monkeys. Taking response latency into account, attentional remapping is

15 well-timed to maintain top-down attention on relevant stimuli, so that they can

16 be tracked and rapidly processed across saccades.

17 


\section{Introduction}

2

3 Humans and monkeys are able to keep track of relevant visual stimuli while

4 making saccadic eye-movements to scan a visual scene. Since the visual

5 system mostly operates using retinotopic representations (Wurtz, 2008;

6 Cavanagh et al., 2010; Marino and Mazer, 2016), in each visual area, a

7 relevant visual stimulus (the target) at a fixed spatial location is represented

8 by one neuronal population before the saccade and a different neuronal

9 population after the saccade: we refer to these as the pre-saccadic target

10 population and the post-saccadic target population respectively. As a result,

11 to maximally and selectively enhance target processing (but not distractor

12 processing) both before and after the saccade, a rapid, saccade-

13 synchronized remapping of top-down attentional modulation from the pre-

14 saccadic to the post-saccadic target population is optimal. Enhancement by

15 top-down spatial attention would ideally be expected to be dominant at the

16 pre-saccadic target population until just before saccade offset, and decay at

17 or soon after saccade offset. Similarly, attentional enhancement would be

18 expected to emerge at the post-saccadic target population at or soon after

19 saccade offset. In other words, if attentional enhancement of the pre-

20 saccadic target population decayed well before the saccade, or attentional

21 enhancement of the post-saccadic target population emerged well after the

22 saccade, there would be time-periods where the target stimulus did not

23 receive the benefits of top-down attention. Contrariwise, if attentional

24 enhancement of the pre-saccadic target population lingered after the

25 saccade, or attentional enhancement of the post-saccadic target population

26 emerged pre-emptively well before the saccade, attention would be peri- 
1 saccadically allocated to irrelevant spatial locations, distractor processing

2 would potentially be facilitated and this would be disadvantageous for task

3 performance.

5 Until now, to our knowledge, the time-course of the remapping of top-down

6 spatial attention from the pre-saccadic to the post-saccadic target population

7 across a saccade has never been explicitly measured. In the only previous

8 physiological recording study on this issue, using a mental curve-tracing task

9 similar to ours with a fixed attentional target, top-down attentional

10 enhancement of multi-unit activity in monkey $\mathrm{V} 1$ was reported to emerge in

11 the post-saccadic target population approximately $80 \mathrm{~ms}$ after the end of the

12 saccade (Khayat et al., 2004). However, this study did not measure the

13 dynamics of the decay of attentional enhancement in the pre-saccadic target

14 population. On the other hand, in a human imaging study, fMRI and EEG

15 data from humans have been presented as evidence for lingering top-down

16 attentional modulation for about $100 \mathrm{~ms}$ after the saccade in the pre-saccadic

17 target population (Golomb et al., 2010a); this was supported by results from

18 human psychophysical studies (Golomb et al., 2008; Golomb et al., 2010b).

19 Human psychophysical data consistent with early, pre-saccadic emergence

20 of top-down attentional modulation in the post-saccadic target population has

21 also been reported (Rolfs et al., 2011; Szinte et al., 2015). This

22 psychophysical inference of pre-emptive attentional modulation in the post-

23 saccadic target population is consistent with a large body of single-neuron

24 recording data from putative attentional control regions in monkeys showing

25 that neurons in the lateral intraparietal area, superior colliculus and frontal

26 eye field (Duhamel et al., 1992; Walker et al., 1995; Umeno and Goldberg, 
1 1997; Wurtz, 2008) respond predictively (and sometimes before the saccade)

2 when a stimulus was expected in their RF after the saccade. This predictive

3 activity is greater for stimuli with greater bottom-up saliency (Gottlieb et al.,

4 1998; Joiner et al., 2011) and for stimuli that are learnt visual search targets

5 (Phillips and Segraves, 2010; Mirpour and Bisley, 2012) or saccade targets

6 (Gottlieb et al., 1998).

7

8 Though these results are suggestive (see Discussion), they do not address

9 the time-course over which top-down attentional enhancement is remapped

10 from the pre-saccadic to the post-saccadic target population across a

11 saccade. In order to measure the time-course of attentional remapping, we

12 trained two monkeys to make a saccade while maintaining top-down

13 attention on moving random dot pattern at a fixed spatial location. We

14 recorded from visual area MT, a key locus in the motion-processing pathway

15 of humans and monkeys, where neurons show both small RFs and clear,

16 robust, attentional enhancement (Treue and Maunsell, 1996, 1999; Busse et

17 al., 2008; Yao et al., 2016a). We show for the first time that attentional

18 remapping is well-sychronized to the saccade and that attentional

19 enhancement crosses over from the pre-saccadic to the post-saccadic target

20 population soon after saccade offset. We recently showed that in humans

21 performing a similar task, top-down spatial attention is fully available at the

22 task-relevant location within 30 milliseconds after the saccade (Yao et al.,

23 2016b). Taking response latency into account (see Discussion), our results

24 show that attentional remapping is well-timed to maintain top-down attention

25 on relevant stimuli, so that they can be tracked and rapidly processed across

26 saccades. 


\section{$1 \quad$ Results}

3 Our task required monkeys to maintain attention on one of four RDPs while

4 also making a saccade (Figure $1 \mathrm{~A}$ ). We recorded from neurons in area MT

5 during this task. Since MT neurons have retinotopic RFs whose spatial

6 location moves with each saccade, the attended target RDP lay in the RF of

7 (and was therefore represented by) different populations of neurons before

8 and after the saccade. In Experiment 1, we estimated the attentional

9 enhancement of the pre-saccadic target population, while in Experiment 2,

10 we estimated the attentional enhancement of the post-saccadic target

11 population. To do this, in Experiment 1, before the saccade, we placed the

12 attended RDP either in the RF (the target condition) or meridionally opposite

13 to it (the distractor condition): we measured the attentional enhancement of

14 the pre-saccadic target population by comparing the firing-rates in the target

15 and distractor conditions. In contrast, in Experiment 2, after the saccade, we

16 placed the attended RDP either in the RF (the target condition) or

17 meridionally opposite to it (the distractor condition): we now measured the

18 attentional enhancement of the post-saccadic target population by comparing

19 the firing-rates in the target and distractor conditions. Based on prior findings

20 (e.g. Treue and Maunsell, 1996, 1999), we expected to see an attentional

21 enhancement of the pre-saccadic target population (in Experiment 1) before

22 the saccade and of the post-saccadic target population (in Experiment 2)

23 after the saccade. This is indeed what we found. In Experiment 1, the

24 population average PSTH showed a greater response before the saccade

25 when a target RDP, rather than a distractor RDP, appeared in the neuron's

26 RF before the saccade (target condition: blue vs. distractor condition: red 
1 curves in Figure 1B); the stimulus in the RF after the saccade was always a

2 distractor. In Experiment 2, the population average PSTH showed a greater

3 response after the saccade when a target RDP, rather than a distractor RDP,

4 appeared in the neuron's RF after the saccade (target condition: blue vs.

5 distractor condition: red curves in Figure 1B); the stimulus in the RF before

6 the saccade was always a distractor. Both these effects were statistically

7 significant. We defined and estimated the attentional enhancement as the

8 difference in firing-rates between target and distractor conditions. For the

9 pre-saccadic target population in Experiment 1, there was significant

10 attentional enhancement in the time-window from 0-500 ms before saccade

11 onset (Monkey $\mathrm{H}$ : mean difference $=5.2 \pm 0.9$ spikes/second, $11.5 \%$

12 enhancement, $p<0.0001$; Monkey $E: 5.9 \pm 1.1$ spikes $/ \mathrm{s}, \quad 31.3 \%$

13 enhancement, $p<0.0001$ ), but not from $0-500 \mathrm{~ms}$ after saccade offset

14 ( $p>0.05)$. For the post-saccadic target population in Experiment 2, there was

15 significant attentional enhancement in the time-window from 0-500 ms after

16 saccade offset (Monkey $\mathrm{H}$ : mean firing-rate difference between target and

17 distractor conditions $=6.0 \pm 0.9$ spikes/second, $19.0 \%$ enhancement,

$18 p<0.0001$; Monkey E: $6.4 \pm 1.1$ spikes $/ s, 20.3 \%$ enhancement $p<0.0001$ ),

19 but not from $0-500$ ms before saccade onset $(p>0.05)$. Further, for both the

20 pre-saccadic and post-saccadic target populations in both monkeys, the

21 difference between the pre-saccadic and the post-saccadic attentional effects

22 was also significantly different (all p-values $<0.001$ ).

24 These results confirm, as expected, that attentional enhancement of firing25 rate is found in the pre-saccadic target population before the saccade, and in 26 the post-saccadic target population after the saccade. Our primary goal was 
1 to characterize the time-course of this remapping of attentional enhancement

2 from the pre-saccadic to the post-saccadic target population and how well

3 this remapping of attentional enhancement was synchronized to the saccade.

4 To do this, we focused on the time-interval from $200 \mathrm{~ms}$ before to $200 \mathrm{~ms}$

5 after saccade offset during which the attentional remapping takes place

6 (Figure 2). For ideal task performance, the attentional remapping would take

7 place as close to saccade offset as possible so that attentional enhancement

8 would be greatest at the pre-saccadic target population until saccade offset

9 and at the post-saccadic target population after saccade offset. We find that

10 attentional enhancement of the pre-saccadic target population (Experiment

11 1) was statistically significant throughout the pre-saccadic period (-200 ms to

$120 \mathrm{~ms}$ before saccade offset) and until $100 \mathrm{~ms}$ of saccade offset in monkey $\mathrm{H}$

13 and $50 \mathrm{~ms}$ in monkey $\mathrm{E}$ (grey curves in Figures $2 \mathrm{~A}, \mathrm{~B}$ ). The attentional

14 enhancement of the post-saccadic target population (Experiment 2) became

15 statistically significant after saccade offset in monkey $\mathrm{H}$ and after $50 \mathrm{~ms}$ of

16 saccade offset in monkey $E$ (black curves in Figures $2 A, B$ ). The attentional

17 effect in the post-saccadic target population became larger than the

18 attentional effect in the pre-saccadic target population at $31 \mathrm{~ms}$ (monkey $\mathrm{H}$,

$19 \mathrm{IQR}=12 \mathrm{~ms}$ ) and $52 \mathrm{~ms}$ (monkey $\mathrm{E}, \mathrm{IQR}=12 \mathrm{~ms}$ ) after saccade offset; we

20 call this time the attentional cross-over time. The proximity of the attentional

21 cross-over to saccade offset indicates that the attentional remapping is well-

22 synchronized to the saccade, after taking the visual response latency of MT

23 neurons into consideration (see Discussion). A direct statistical comparison

24 of the attentional effects in Experiment 1 and 2 also confirmed this result.

25 The attentional effect in the pre-saccadic target population (Experiment 1,

26 grey curve in Figures $2 A, B$ ) was significantly greater than that in the post- 
1 saccadic target population (Experiment 1, grey curve in Figures 2A,B) until

2 saccade offset in monkey $\mathrm{H}$ and $50 \mathrm{~ms}$ after saccade offset in monkey $\mathrm{E}$

3 (two-sample t-test, one-sided $p$-value $<0.05$ ). This effect then reversed: the

4 attentional effect in the post-saccadic target population became greater than

5 that in the pre-saccadic target population after $50 \mathrm{~ms}$ following saccade

6 offset in both monkeys.

7

8 These results indicate that attentional remapping was well synchronized with

9 saccade planning/execution. We examined the alternative possibility that 10 attentional remapping and saccade planning/execution were both triggered 11 by the cue to make the saccade, but proceeded independently. If attentional remapping and saccade planning/execution proceed independently, then relative to saccade offset, the time-course of attentional remapping would be delayed for trials with short-latency saccades compared to trials with longlatency saccades. On the other hand, if attentional remapping and saccade planning/execution are indeed synchronized with each other, then the timecourse of attentional remapping would be similar for short and long-latency saccades. At the same time, in this scenario, relative to the cue to make the saccade (i.e. the offset of the fixation point), attentional remapping would occur later on trials with long-latency saccades compared to short-latency saccades. We therefore plotted the time-course of attentional remapping aligned to saccade offset (Fig.3A,C) and fixation point offset (Fig.3B,D) for trials with saccade latencies shorter than the $33^{\text {rd }}$ percentile and longer than the $66^{\text {th }}$ percentile (solid and dashed curves respectively; also see Supplementary Figure 2). We found the pattern expected if attentional remapping was synchronized with saccade planning/execution. For both 
1 Experiment 1 (Fig.3A,B) and Experiment 2 (Fig.3C,D), when aligned to

2 saccade offset, the the remapping time-course for trials with long-latency

3 saccades and trials with short-latency saccades was superimposed;

4 consistent with the visual impression, the peak cross-correlation lag was at

5 zero delay. Similarly, as expected, when aligned to fixation point offset (i.e.

6 the cue to make the saccade), the remapping time-course for trials with long-

7 latency saccades was delayed compared to the trials with short-latency

8 saccades in both Experiment 1 and 2, with a peak cross-correlation lag of 50

9 ms. However, the pattern of a delay when aligned to fixation point offset is

10 especially clear for the attentional enhancement of the post-saccadic

11 population (in Experiment 2). In Experiment 1 (for the attentional decay in the

12 pre-saccadic population), the delay remains clear at times after the mean

13 saccade latency (Figure 3B), but the curves appear superimposed before

14 that. It therefore remains possible that the early time-course of attentional

15 decay in the pre-saccadic population was triggered by saccade offset, and

16 then became co-ordinated with saccade execution in the post-saccadic

17 phase. The data from individual monkeys, though noisy (because each curve

18 now used only one-third of the trials from the corresponding task condition)

19 was consistent with these general patterns (Supplementary Figures 3 and 4).

21 We considered two additional issues. First, we did not find any evidence for

22 predictive attentional remapping in $\mathrm{MT}$ : there was no attentional

23 enhancement of the post-saccadic target population before saccade offset

24 (black diamonds in Figures $2 A, B$ ). This is particularly notable because unlike 25 earlier studies (Ong and Bisley, 2011; Yao et al., 2016a), we made sure that 26 there was a stimulus in the RF before the saccade. The presence of this 
1 stimulus ensured a stimulus-driven response on which a putative predictive

2 attentional signal could act, and rules out the argument that the apparent

3 absence of a predictive response is simply because the predictive attentional

4 signal does not modulate spontaneous activity in MT. Second, we considered

5 the fact that in monkey $\mathrm{E}$, the firing-rates of the neurons in both the target

6 and distractor conditions is higher in Experiment 2 (Figure 2D) than in

7 Experiment 1 (Figure 2F), even though two-sample tests did not show a

8 significant difference. To rule out a potential influence of this difference on

9 our estimate of the attentional time-course in monkey E, we dropped the 25

$10 \%$ of neurons with the highest overall firing rates in Experiment 2 and

11 restricted the analysis to the remaining $75 \%$ of neurons (Supplementary

12 Figure 1). This procedure substantially equates the firing-rates in the two

13 Experiments, but does not affect our conclusions. The attentional cross-over

14 time now occurs at a median time of $56 \mathrm{~ms}$ (IQR =13 ms) after saccade

15 offset (compared to $52 \mathrm{~ms}, \mathrm{IQR}=12 \mathrm{~ms}$ in the full sample in Figure 2B). 
3 We report, for the first time, that attentional remapping is well-sychronized to

4 the saccade: attentional enhancement crosses over from the pre-saccadic to

5 the post-saccadic target population at 31 and $52 \mathrm{~ms}$ after saccade offset in

6 the two monkeys. We recently showed that in humans performing a similar

7 task, top-down spatial attention is fully available at the task-relevant location

8 within 30 milliseconds after the saccade (Yao et al., 2016b). Given an onset

9 latency of approximately $30 \mathrm{~ms}$ in MT (Bair et al., 2002), a visual change

10 occurring $30 \mathrm{~ms}$ after saccade offset would reach MT by $60 \mathrm{~ms}$ after saccade

11 offset, by which time attention would have crossed-over to the post-saccadic

12 target population (as we show here). This rapid recovery of spatial attention

13 following a saccade is also consistent with data from double-step saccades

14 (Hallett and Lightstone, 1976) and visual search (McPeek et al., 2000)

15 showing that successive saccades can be made with very short inter-

16 saccadic intervals and therefore the target of the second saccade can be

17 rapidly located after the first saccade. In our data, attentional enhancement

18 of the post-saccadic target population emerged at saccade offset and within

$1950 \mathrm{~ms}$ of saccade offset in the two monkeys, compared to the $80 \mathrm{~ms}$ time

20 (after the stimulus entered the post-saccadic RF) reported in a previous

21 study of multi-unit activity in V1 in monkeys performing a mental curve-

22 tracking task (Khayat et al., 2004). Given the uncertainty in estimating the RF

23 edge during a saccade, the times in the two studies appear comparable (or

24 possibly slightly earlier in MT). Also, in our data, attentional enhancement of

25 the pre-saccadic target population lingered after the saccade and

26 disappeared by $50 \mathrm{~ms}$ after saccade offset in one monkey and by $100 \mathrm{~ms}$ in 
1 the other. This is consistent with previous human psychophysical and

2 imaging evidence suggesting that a lingering attentional modulation of the

3 pre-saccadic target population for about $100 \mathrm{~ms}$ after saccade offset

4 (Golomb et al., 2008; Golomb et al., 2010a; Golomb et al., 2010b). The

5 lingering attentional modulation at the pre-saccadic neuronal population even

6 after attentional effects have emerged at the post-saccadic neuronal

7 population is reminiscent of similar effects observed in attentional switch

8 experiments where monkeys covertly changed their locus of spatial attention

9 while maintaining fixation: in that scenario, attentional effects emerge at the

10 new locus of spatial attention (and the neuronal population encoding this

11 locus) before attentional effects decay in the neuronal population encoding

12 the preceding locus of attention (Khayat et al., 2006; Busse et al., 2008). The

13 time taken to accomplish the attentional switch in these previous studies in

14 V1, MT and LIP, which can be as short as 150-200 ms (Khayat et al., 2006;

15 Busse et al., 2008; Herrington and Assad, 2009, 2010), is also comparable to

16 the time-course of attentional remapping across a saccade in our task. Our

17 analyses however showed that attentional remapping across a saccade is

18 synchronized to saccade planning/execution and is not the result of a visual

19 cue-induced attentional switch as in these previous studies. It is possible that

20 covert volitional attentional switches during fixation may engage the same

21 circuitry as attentional remapping across saccades. The close co-ordination

22 between peri-saccadic attentional remapping and saccadic

23 planning/execution may be facilitated by the overlapping neural circuitry

24 mediating these two phenomena (Awh et al., 2006; Noudoost et al., 2010). 
1 We did not find any evidence for attentional enhancement of the post-

2 saccadic target population before the saccade, even though our experimental

3 design ensured that there would be a distractor-driven response before the

4 saccade on which an attentional effect could be seen, if present. This

5 confirms results in two previous studies (Ong and Bisley, 2011; Yao et al.,

6 2016a) where no significant attentional modulation of spontaneous activity

7 was found in MT neurons before the saccade. Similarly, in our recent human

8 psychophysical study using a stimulus paradigm very similar to the one here,

9 we did not find any evidence for a predictive, pre-saccadic shift of attention to

10 the post-saccadic target population (Yao et al., 2016b). In contrast, a large

11 body of single-neuron recording data from putative attentional control regions

12 in monkeys shows that neurons in the lateral intraparietal area, superior

13 colliculus and frontal eye field (Duhamel et al., 1992; Walker et al., 1995;

14 Umeno and Goldberg, 1997; Wurtz, 2008; Mirpour and Bisley, 2016) as well

15 as some ventral stream areas (Nakamura and Colby, 2002) respond

16 predictively (and sometimes before the saccade) when their RF was

17 stimulated before the saccade, but not after the saccade. Though this

18 anticipatory activity has not been studied explicitly in conditions evoking top-

19 down spatial attention, predictive activity is greater for stimuli with greater

20 bottom-up saliency (Gottlieb et al., 1998; Joiner et al., 2011) and for stimuli

21 that are learnt visual search targets (Phillips and Segraves, 2010; Mirpour

22 and Bisley, 2012) or saccade targets (Gottlieb et al., 1998). Human

23 psychophysical data consistent an with early, pre-saccadic emergence of

24 top-down attentional modulation in the post-saccadic target population have

25 also been reported (Rolfs et al., 2011; Szinte et al., 2015). We hypothesize

26 that the anticipatory remapping seen in attentional and oculomotor control 
1 areas like LIP, FEF and SC is part of the process that enables attentional

2 remapping in MT that is well synchronized to the saccade. In other words,

3 even though this process starts before the saccade in these areas, its effects

4 in MT, with which these areas are strongly connected (Maunsell and van

5 Essen, 1983; Ungerleider and Desimone, 1986; Blatt et al., 1990), only

6 manifest after the saccade. In this view, the previous results on trans-

7 saccadic remapping represent the predictive, pre-saccadic shift of attentional

8 pointers on a retinotopic map that keeps track of attended locations across

9 saccades (Cavanagh et al., 2010), so that attended locations can be

10 preferentially processed with minimal delay after the saccade (Yao et al.,

11 2016a). This reduction of delay would be especially helpful when planning

12 rapid sequential saccades and could also help maintain an uninterrupted

13 visual experience across saccades. While additional evidence from other

14 visual areas (for example, in the ventral stream) and using other visual

15 stimuli are undoubtedly needed to resolve these issues, the physiological

16 data here, combined with our recent human psychophysics results (Yao et

17 al., 2016b) support our hypothesis that spatial attention and saccadic

18 processing co-ordinate well to ensure that relevant locations are attentionally

19 enhanced soon after the beginning of each eye fixation, and can be tracked

20 and rapidly processed across saccades. 


\section{Experimental Procedures}

2

3 Our description of the Methods here is similar to that presented in our

4 previous publication (Yao et al., 2016a), since the general experimental

5 procedures are the same. We trained two male rhesus monkeys (Macaca

6 mulatta, 7-11 kg), monkey $\mathrm{H}$ and monkey $\mathrm{E}$, to perform a demanding

7 visuospatial-attention task along with a saccade. Each monkey was

8 implanted with a titanium head holder to minimize head movements during

9 the experiment. One recording chamber was also implanted in each monkey

10 above the left (monkey E) or the right (monkey $\mathrm{H}$ ) parietal cortex to allow

11 access to MT, with implantation locations chosen based on a preceding MRI

12 scan. All procedures were approved by the district government of Lower

13 Saxony, Germany, and all surgeries were conducted under general

14 anesthesia using standard techniques.

15

16 The experiments were performed in a dimly-lit room with the only source of

17 light being the display monitor. A CRT monitor (Sony Trinitron GDM-FW900)

18 at a distance of $57 \mathrm{~cm}$ from the monkey was used to display the visual

19 stimulus at a refresh rate of $76 \mathrm{~Hz}$ and a spatial resolution of 40

20 pixels/degree. The monkey sat in a custom-made primate chair during the

21 experiment. Stimulus presentation, reward delivery, electrophysiological and

22 behavioral data collection was controlled by custom software and run on an

23 Apple Macintosh computer. All stimulus onsets and durations were specified

24 in terms of number of frames (CRT monitor refreshes), and the stimulus

25 presentation times reported here in millisecond units are correct to within 13

$26 \mathrm{~ms}$ (the duration of one frame), given the vertical scan-rate properties of the 
1 CRT monitor. The animals received a fluid reward immediately following

2 each correct trial. The eye-position was monitored by an EyeLink 1000 (SR

3 Research, Canada) system at $1000 \mathrm{~Hz}$. Neuronal activity was recorded

4 extracellularly with a 5-channel micro drive system (Mini Matrix, Thomas

5 Recording, Giessen, Germany) and processed using the Plexon data

6 acquisition system (Plexon Inc., Dallas, TX). Only data from well-isolated

7 neurons were used for the analysis. MT was identified by referencing the

8 recordings to the structural MRI and by the physiological properties of the

9 recorded neurons: most neurons were direction-tuned, the average diameter

10 of the receptive fields (RFs) was approximately equal to the RF eccentricity

11 and there was a predictable progression of RF centers at different locations

12 along the superior temporal sulcus.

13

14

17 Once a neuron was isolated, we had the monkey perform a fixation task 18 where the monkey had to maintain fixation on a fixation point and respond to 19 a brief luminance change at the fixation point. During this fixation period, we 20 located the RF by moving a stationary circular random dot pattern (RDP) 21 across the screen using a mouse. We then determined the neuron's 22 preferred direction and speed during the fixation task by presenting a RDP 23 with moving dots within a circular aperture in the RF, changing the direction 24 and speed every $250 \mathrm{~ms}$ to a value picked from a set of 3 possible speeds 25 (4, 8 or 16 degrees per second) and 12 possible directions (evenly separated 26 by 30 degrees around a circle). For the main experiment, we used stimuli 
1 with directions and speeds equal or close to the preferred direction and the

2 preferred speed thus determined.

4 After identifying the RF location and preferred direction, we switched to the

5 main experiment (Figure 1), where the monkey performed an experimental

6 "attention-saccade" task (either Experiment 1 or Experiment 2) and a control

7 task in a pseudo-randomly interleaved manner. In the experimental task,

8 comprising $80 \%$ of trials, the monkeys were trained to concurrently perform

9 a visuospatial attention task and a saccade task on each trial: they were

10 instructed to pay attention to the target RDP and make a saccade if the

11 fixation point jumped to a new location. The monkeys initiated the trial by

12 holding a metal bar and foveating a black fixation point. After $118 \mathrm{~ms}$ of

13 fixation, to indicate the location of the upcoming target RDP, a stationary

14 circular RDP cue (of the same size as the target) was presented for $263 \mathrm{~ms}$.

15 After an additional delay of 329 ms following cue offset, four moving RDPs (2

16 degrees in radius, all dots moving at or close to the neuron's preferred

17 direction of motion and within stationary circular apertures) were presented

18 on the screen. Two of the RDPs were presented in the neuron's pre-saccadic

19 and post-saccadic RFs respectively and the other two RDPs were located

20 opposite to these stimuli (i.e. reflected across the horizontal or vertical

21 meridian, see Figure 1). The monkeys' task was to respond to a brief (132

$22 \mathrm{~ms}$ ) direction change in the RDP at the previously cued location (the target)

23 by releasing the metal bar (within $600 \mathrm{~ms}$ of the change), but ignore similar

24 changes in the distractor (the RDP opposite to the target). Trials terminated

$25600 \mathrm{~ms}$ after the target change, with the monkey receiving a drop of juice if

26 the bar had been correctly released during this period. In addition, during the 
1 trial, if the fixation point jumped to a new location, the monkeys had to re-

2 fixate the fixation point while continuing to attend to the cued target. The

3 direction change in the target RDP could occur between $263 \mathrm{~ms}$ to $1973 \mathrm{~ms}$

4 after RDP onset. Distractor changes occurred on about $37 \%$ of trials and

5 never more than once on each trial. The timing of distractor changes

6 overlapped that of target changes, with the additional requirement that any

7 distractor change occurred at least $500 \mathrm{~ms}$ before the target change on each

8 trial. This separation ensured that the monkeys' rare responses to the

9 distractor change could be easily identified and distinguished from their

10 responses to the target change. The fixation point jumped to its new location

11 (and became the saccade target) $1382 \mathrm{~ms}$ after fixation point onset (i.e. 671

12 ms after RDP onset); however, this event did not occur if the trial had

13 terminated by then (either by a correct or incorrect bar-release or by a

14 missed target change). The saccade target then stayed on for $1368 \mathrm{~ms}$ (or

15 until the end of the trial). There was a one-frame (13 ms) overlap between

16 the fixation point and the saccade target, so that the fixation point

17 disappeared one frame after the saccade target appeared: perceptually, the

18 fixation point appeared to jump from its original location to the saccade

19 target. Once the fixation point jumped, the monkey had to make a saccade to

20 the new location of the fixation point within $263 \mathrm{~ms}$ and maintain fixation until

21 the end of the trial. The saccade target appeared between 10 and 20

22 degrees eccentrically (value fixed for each neuron, and either 15 or 20

23 degrees in most cases). Saccades were always either horizontal or vertical.

24 We used a fixed and predictable time for the fixation point jump to reduce the

25 temporal uncertainty about when the fixation point would jump and thereby

26 minimize the monkeys' need to monitor the fixation point or saccade target 
1 location in order to detect the saccade jump. This would enable the monkeys

2 to better focus their attention on the target RDP. The median saccade

3 latency was $136 \mathrm{~ms}$ in monkey $\mathrm{H}$ and $142 \mathrm{~ms}$ in monkey $\mathrm{E} ; 99 \%$ of the

4 saccades occurred before $217 \mathrm{~ms}$ in monkey $\mathrm{H}$ and $229 \mathrm{~ms}$ in monkey $\mathrm{E}$.

6 In Experiment 1, the cue (and by extension, the target RDP) was located

7 either in the neurons' pre-saccadic RF (attend-in condition) or opposite to it

8 (attend-out condition) equally often in a pseudo-randomly interleaved

9 manner. Experiment 2 was similar, except that the cue (and by extension, the

10 target RDP) was located either in the neurons' post-saccadic RF (attend-in

11 condition) or opposite to it (attend-out condition) equally often in a pseudo-

12 randomly interleaved manner. The control task, comprising $20 \%$ of trials,

13 was a 'simple-saccade' task where the monkey simply made a saccade

14 when the fixation point jumped to a new location and maintained fixation till

15 the end of the trial to obtain the juice reward. There was no concurrent

16 attentional task; i.e. no cue and no moving RDPs were presented. Data from

17 this control task were only used to select visually-responsive neurons for

18 further analysis (see below).

20 In all the tasks, the background was always grey with a luminance of 14.2

$21 \mathrm{~cd} / \mathrm{m}^{2}$, and the fixation point and RDPs including the stationary cue were

22 black with the luminance of $0.68 \mathrm{~cd} / \mathrm{m}^{2}$. Individual RDP dot size was $0.1^{\circ} \mathrm{x}$

$230.1^{\circ}$, and the dot density was $10 \mathrm{dots} / \mathrm{deg}^{2}$. Monkeys had to maintain fixation

24 within a circular window of 2 degrees radius around the fixation point before

25 the fixation point jumped. Following a period of $263 \mathrm{~ms}$ after the fixation point

26 jumped (which gave the monkeys time to make the saccade), the monkeys 
1 had to maintain fixation within a circular window of 3 degrees radius around

2 the saccade target. This larger post-saccadic window was not necessitated

3 by fixation inaccuracy, but rather by across-trial drifts in calibration: using a

4 window centered on the median eye-position within the trial, the maximal

5 within-trial deviation was less than 2 degrees in over $95 \%$ of trials. The

6 saccade direction was set according to the position of the RF: for example, if

7 the RF center was directly above or below the fixation point, we used a

8 horizontal saccade, while if the RF center was directly to the left or right of

9 the fixation point, we used a vertical saccade. If the RF center was offset

10 both vertically and horizontally from the fixation point, the choice was no

11 longer critical, but we usually used a horizontal saccade.

12

13

14

15

16 All data analysis was performed using custom software in MATLAB 17 (MATLAB Inc, Natick, MA). We detected saccades using a velocity-threshold

Data Analysis criterion that was validated by visual inspection. onset (and offset) times were determined by when the eye velocity exceeded (and then dropped below) 100 degrees per second. This threshold value was set to lie clearly above the peak excursions of the baseline noise in the eye-velocity traces, and the algorithm was validated by visual inspection for each monkey. By considering the saccade to have ended when the velocity dropped below a threshold value well above the baseline noise (and when the eye was still moving), our threshold criterion provides a conservative, i.e. early definition of saccadic end-point and therefore if anything, a longer estimate of the cross-over time for attentional remapping. 
2 We included data from all neurons that showed a visual response to the RDP

3 in the RF both before and after the saccade. We identified these neurons as

4 those that showed a significantly greater postsaccadic response (one-sided t-

5 test, $p<0.05$, Bonferroni-corrected) in the attention-saccade task compared to

6 the simple-saccade control in the time-periods 0 to $600 \mathrm{~ms}$ following RDP

7 onset (i.e. they were visually responsive to the RDP in the pre-saccadic RF)

8 and 0 to 600 ms following the saccade (i.e. they were visually responsive to

9 the RDP in the post-saccadic RF). In total, out of 123 and 137 neurons

10 recorded in Experiment 1 and Experiment 2 respectively, we analyzed 84

11 neurons in Experiment 1 (56 in monkey $\mathrm{H}$ and 28 in monkey $\mathrm{E}$ ) and 84

12 neurons in Experiment 2 (52 neurons in monkey $\mathrm{H}$ and 32 neurons in

13 monkey E). 29 neurons (all in monkey H) provided data for both Experiments

141 and 2. There were at least 9 trials for both the attend-in and attend-out

15 conditions in Experiment 1 and at least 14 trials in Experiment 2 in all

16 neurons. After excluding fixation breaks, monkey $\mathrm{H}$ performed the task

17 correctly on $93.2 \%$ of trials (with early "false-positive" releases on $3.7 \%$ and

18 "misses" on $3.1 \%$ of trials), while monkey E performed the task correctly on

$1984.6 \%$ of trials (with false-positive releases on $8.8 \%$ and misses on $6.6 \%$

20 of trials). We analyzed all correctly completed trials where the target change

21 did not occur too close to saccade offset (i.e. the target change was at least

22200 ms earlier or 200 ms later than saccade offset), since this was the time-

23 period that we focused on to measure the time-course of attentional

24 remapping.

25

26 Peri-stimulus time histograms (PSTHs) were calculated using non- 
1 overlapping $25 \mathrm{~ms}$ time bins. The mean activity for each neuron across trials

2 was first calculated and then these mean PSTHs for individual neurons were

3 averaged across neurons to obtain the displayed PSTHs. In the attend-out

4 conditions, to avoid the transient response to the brief change in the

5 distractor stimulus within the receptive field, we excluded the period of 0 to

$6350 \mathrm{~ms}$ following the distractor change from the PSTH and firing-rate

7 calculations. To calculate percentage changes in firing-rate when comparing

8 two conditions, we first calculated a modulation index for each neuron as the

9 difference in the firing-rates for the two conditions divided by their sum,

10 averaged the modulation indices and then converted the average back into a

11 percentage change. We used two-sided t-tests throughout unless mentioned

12 otherwise; using the signed-rank test produced similar results. To calculate

13 the attentional cross-over time, we used linear interpolation between data

14 points to compute the time at which the black and grey curves (in Figures $2 \mathrm{~A}$,

$152 \mathrm{~B}$ and Supplementary Figure 1) crossed. To characterize the variability of

16 this cross-over time, we used a bootstrap procedure where we randomly

17 selected neurons with replacement to compute the attentional effect curves

18 in the two experiments, computed the cross-over time and repeated this

19 procedure 15000 times to generate a distribution of cross-over times. Since

20 this distribution was skewed, we used the inter-quartile range (IQR) of this

21 distribution to characterize the variability of the cross-over time. The median

22 of the bootstrap distribution was very close in value to the cross-over time

23 calculated from the original dataset. To estimate the delay between the

24 difference curves in Figure 3 (and Supplemental Figure 3), we found the

25 time-lag at which the cross-correlation reached a maximum value; this time-

26 lag was consistent with the visual impression based on the graph. 


\section{Author Contributions}

2

3 TY and BSK designed the study; TY conducted the experiments; TY and

$4 \quad$ BSK analyzed the data; ST commented on the manuscript; TY and BSK

5 wrote the manuscript.

6

7 Acknowledgments

8

9 The study was funded by grants to Stefan Treue from the Deutsche

10 Forschungsgemeinschaft (DFG): Collaborative Research Center 889

11 "Cellular Mechanisms of Sensory Processing" (Project C04) and the

12 German-Israeli Foundation for Scientific Research and Development (GIF):

$13 \quad 1108-79.1 / 2010$

14 


\section{References}

Awh E, Armstrong KM, Moore T (2006) Visual and oculomotor selection: links, causes and implications for spatial attention. Trends in cognitive sciences 10:124-130.

Bair W, Cavanaugh JR, Smith MA, Movshon JA (2002) The timing of response onset and offset in macaque visual neurons. The Journal of neuroscience : the official journal of the Society for Neuroscience 22:3189-3205.

Blatt GJ, Andersen RA, Stoner GR (1990) Visual receptive field organization and cortico-cortical connections of the lateral intraparietal area (area LIP) in the macaque. The Journal of comparative neurology 299:421-445.

Busse L, Katzner S, Treue S (2008) Temporal dynamics of neuronal modulation during exogenous and endogenous shifts of visual attention in macaque area MT. Proceedings of the National Academy of Sciences of the United States of America 105:16380-16385.

Cavanagh P, Hunt AR, Afraz A, Rolfs M (2010) Visual stability based on remapping of attention pointers. Trends in cognitive sciences 14:147153.

Duhamel JR, Colby CL, Goldberg ME (1992) The updating of the representation of visual space in parietal cortex by intended eye movements. Science 255:90-92.

Golomb JD, Chun MM, Mazer JA (2008) The native coordinate system of spatial attention is retinotopic. The Journal of neuroscience : the official journal of the Society for Neuroscience 28:10654-10662.

Golomb JD, Nguyen-Phuc AY, Mazer JA, McCarthy G, Chun MM (2010a) Attentional facilitation throughout human visual cortex lingers in retinotopic coordinates after eye movements. The Journal of neuroscience : the official journal of the Society for Neuroscience 30:10493-10506.

Golomb JD, Pulido VZ, Albrecht AR, Chun MM, Mazer JA (2010b) Robustness of the retinotopic attentional trace after eye movements. Journal of vision 10:19 11-12.

Gottlieb JP, Kusunoki M, Goldberg ME (1998) The representation of visual salience in monkey parietal cortex. Nature 391:481-484.

Hallett PE, Lightstone AD (1976) Saccadic eye movements towards stimuli triggered by prior saccades. Vision research 16:99-106.

Herrington TM, Assad JA (2009) Neural activity in the middle temporal area and lateral intraparietal area during endogenously cued shifts of attention. The Journal of neuroscience : the official journal of the Society for Neuroscience 29:14160-14176.

Herrington TM, Assad JA (2010) Temporal sequence of attentional modulation in the lateral intraparietal area and middle temporal area during rapid covert shifts of attention. The Journal of neuroscience : the official journal of the Society for Neuroscience 30:3287-3296.

Joiner WM, Cavanaugh J, Wurtz RH (2011) Modulation of shifting receptive field activity in frontal eye field by visual salience. Journal of neurophysiology 106:1179-1190.

Khayat PS, Spekreijse H, Roelfsema PR (2004) Correlates of transsaccadic integration in the primary visual cortex of the monkey. Proceedings of 
the National Academy of Sciences of the United States of America 101:12712-12717.

Khayat PS, Spekreijse H, Roelfsema PR (2006) Attention lights up new object representations before the old ones fade away. The Journal of neuroscience : the official journal of the Society for Neuroscience 26:138142.

Marino AC, Mazer JA (2016) Perisaccadic Updating of Visual Representations and Attentional States: Linking Behavior and Neurophysiology. Frontiers in systems neuroscience 10:3.

Maunsell JH, van Essen DC (1983) The connections of the middle temporal visual area (MT) and their relationship to a cortical hierarchy in the macaque monkey. The Journal of neuroscience : the official journal of the Society for Neuroscience 3:2563-2586.

McPeek RM, Skavenski AA, Nakayama K (2000) Concurrent processing of saccades in visual search. Vision research 40:2499-2516.

Mirpour K, Bisley JW (2012) Anticipatory remapping of attentional priority across the entire visual field. The Journal of neuroscience : the official journal of the Society for Neuroscience 32:16449-16457.

Mirpour K, Bisley JW (2016) Remapping, Spatial Stability, and Temporal Continuity: From the Pre-Saccadic to Postsaccadic Representation of Visual Space in LIP. Cerebral cortex 26:3183-3195.

Nakamura K, Colby CL (2002) Updating of the visual representation in monkey striate and extrastriate cortex during saccades. Proceedings of the National Academy of Sciences of the United States of America 99:40264031.

Noudoost B, Chang MH, Steinmetz NA, Moore T (2010) Top-down control of visual attention. Current opinion in neurobiology 20:183-190.

Ong WS, Bisley JW (2011) A lack of anticipatory remapping of retinotopic receptive fields in the middle temporal area. The Journal of neuroscience : the official journal of the Society for Neuroscience 31:10432-10436.

Phillips AN, Segraves MA (2010) Predictive activity in macaque frontal eye field neurons during natural scene searching. Journal of neurophysiology 103:1238-1252.

Rolfs M, Jonikaitis D, Deubel H, Cavanagh P (2011) Predictive remapping of attention across eye movements. Nature neuroscience 14:252-256.

Szinte M, Carrasco M, Cavanagh P, Rolfs M (2015) Attentional trade-offs maintain the tracking of moving objects across saccades. Journal of neurophysiology 113:2220-2231.

Treue S, Maunsell JH (1996) Attentional modulation of visual motion processing in cortical areas MT and MST. Nature 382:539-541.

Treue S, Maunsell JH (1999) Effects of attention on the processing of motion in macaque middle temporal and medial superior temporal visual cortical areas. The Journal of neuroscience : the official journal of the Society for Neuroscience 19:7591-7602.

Umeno MM, Goldberg ME (1997) Spatial processing in the monkey frontal eye field. I. Predictive visual responses. Journal of neurophysiology 78:13731383.

Ungerleider LG, Desimone R (1986) Cortical connections of visual area MT in the macaque. The Journal of comparative neurology 248:190-222. 
Walker MF, Fitzgibbon EJ, Goldberg ME (1995) Neurons in the monkey superior colliculus predict the visual result of impending saccadic eye movements. Journal of neurophysiology 73:1988-2003.

Wurtz RH (2008) Neuronal mechanisms of visual stability. Vision research 48:2070-2089.

Yao T, Treue S, Krishna BS (2016a) An Attention-Sensitive Memory Trace in Macaque MT Following Saccadic Eye Movements. PLoS Biol 14:e1002390.

Yao T, Ketkar M, Treue S, Krishna BS (2016b) Visual attention is available at a task-relevant location rapidly after a saccade. eLife In press. 
1 Figure Legends

2

3 Figure 1. Attention enhances different target populations before and

4 after a saccade. A) Cartoon demonstrating that in a retinotopic

5 representation, an attended stimulus is represented in different populations

6 of neurons before and after a saccade. Therefore, to maintain attention on a

7 stimulus while making a saccade, attentional effects must manifest in

8 different populations of neurons before and after the saccade. B) Task-

9 design and timing: Two rhesus monkeys were trained to perform a task that

10 involved attending to one of four moving RDPs (the target) while also making

11 a visually guided saccade if the fixation point (FP) jumped to a new location.

12 An initial spatial cue marked the target location on each trial. The intervening

13 saccade changed the spatial location of the neuron's RF so that a different

14 RDP appeared in the RF before and after the saccade. In Experiment 1, the target RDP appeared before the saccade either in the neuron's RF or at the meridionally opposite location. In Experiment 2, the same happened after the saccade. Values next to each panel represent the durations of the task phase represented by that panel. For details, see Materials and Methods. C) Population average peri-stimulus time histograms (PSTHs) in Experiment 1 aligned to the time of saccade offset. After the saccade, the stimulus in the RF was always a distractor, while before the saccade, either a target (blue curve) or a distractor (red curve) appeared in the neuron's RF: the neurons respond more in the former case before the saccade. PSTHs plotted using $25 \mathrm{~ms}$, non-overlapping bins. The inset rectangles depict the cue location for the two conditions. From left to right, the first dashed vertical line represents the mean time of RDP onset, the second dashed vertical line the mean time 
1 of fixation point jump and the dotted vertical line the mean time of saccade

2 onset. The early response before RDP onset in the attend-in condition (blue

3 curve) is the response to the cue. Data pooled from both monkeys. D)

4 PSTHs for Experiment 2, in format a similar to B. Before the saccade, the

5 stimulus in the RF was always a distractor, while after the saccade, either a

6 target (blue curve) or a distractor (red curve) appeared in the neuron's RF:

7 the neurons respond more in the former case after the saccade.

9 Figure 2. Attentional remapping is well-synchronized to the saccade.

$10 \mathrm{~A}, \mathrm{~B}$ ) The attentional cross-over time (when the attentional enhancement of

11 the post-saccadic target population became larger than the attentional 12 enhancement of the pre-saccadic target population) occurred at $31 \mathrm{~ms}$ (A, $13 \mathrm{IQR}=12 \mathrm{~ms}$, monkey $\mathrm{H})$ and $52 \mathrm{~ms}(\mathrm{~B}, \mathrm{IQR}=12 \mathrm{~ms}$, monkey $\mathrm{E})$ after 14 saccade offset. Data for monkey $\mathrm{H}$ in $\mathrm{A}$ and for monkey $\mathrm{E}$ in $\mathrm{B}$. Grey and 15 black curves show the mean difference (and SEMs) between the target-in-RF 16 and distractor-in-RF curves in Experiment 1 and Experiment 2 respectively 17 (as shown in $\mathrm{C}-\mathrm{F}$ ), but plotted using $50 \mathrm{~ms}$, non-overlapping time-bins. 18 Diamonds above the curves indicate the successive, non-overlapping $50 \mathrm{~ms}$ 19 time-bins in which the differences were significantly larger than zero (one20 sided t-test): black diamonds for the black curve and gray diamonds for the 21 gray curve. C-F) Same data as in Figure 1B (C-D) and Figure 1C (E-F), but 22 plotted separately for the two monkeys and focusing on the time around the 23 saccade (-200 to $200 \mathrm{~ms}$ relative to saccade offset); format otherwise 24 identical. The gray curves in $A$ and $B$ are computed as the difference 25 between the blue and red curves in $C$ and $D$ respectively, while the black 
1 curves in $A$ and $B$ are computed as the difference between the blue and red

2 curves in E and F respectively. Also see Supplemental Figure 1.

4 Figure 3. Attentional remapping is synchronized to the saccade, not to

5 fixation point offset. The time-course of remapping, plotted aligned to

6 saccade offset $(A, C)$ is similar for trials with saccade latencies shorter than

7 the $33^{\text {rd }}$ percentile (solid curves) and longer than the $66^{\text {th }}$ percentile (dashed

8 curves) for the corresponding task condition, but is delayed $(B, D)$ for the

9 longer-latency trials by one bin $(50 \mathrm{~ms})$ when plotted aligned to fixation point

10 offset: the delay was calculated as the cross-correlation lag that yielded a

11 maximum. The delay when aligned to saccade offset $(A, C)$ was 0.

12 Remapping time-course defined as in Figure 3A.B as the difference between

13 the firing-rates in the attend-in and attend-out conditions (see Supplementary

14 Figure 2 for the corresponding PSTHs). Results for Experiment 1 in the top

15 row $(A, B)$ and for Experiment 2 in the bottom row $(C, D)$. Solid and dashed

16 vertical lines in each panel represent the mean time of fixation-point offset

$17(A, C)$ or saccade latency $(B, D)$ for trials with short-latency and long-latency

18 saccades respectively. Data from both monkeys were pooled for this analysis

19 (see Text and Supplemental Figures 2 and 3). 

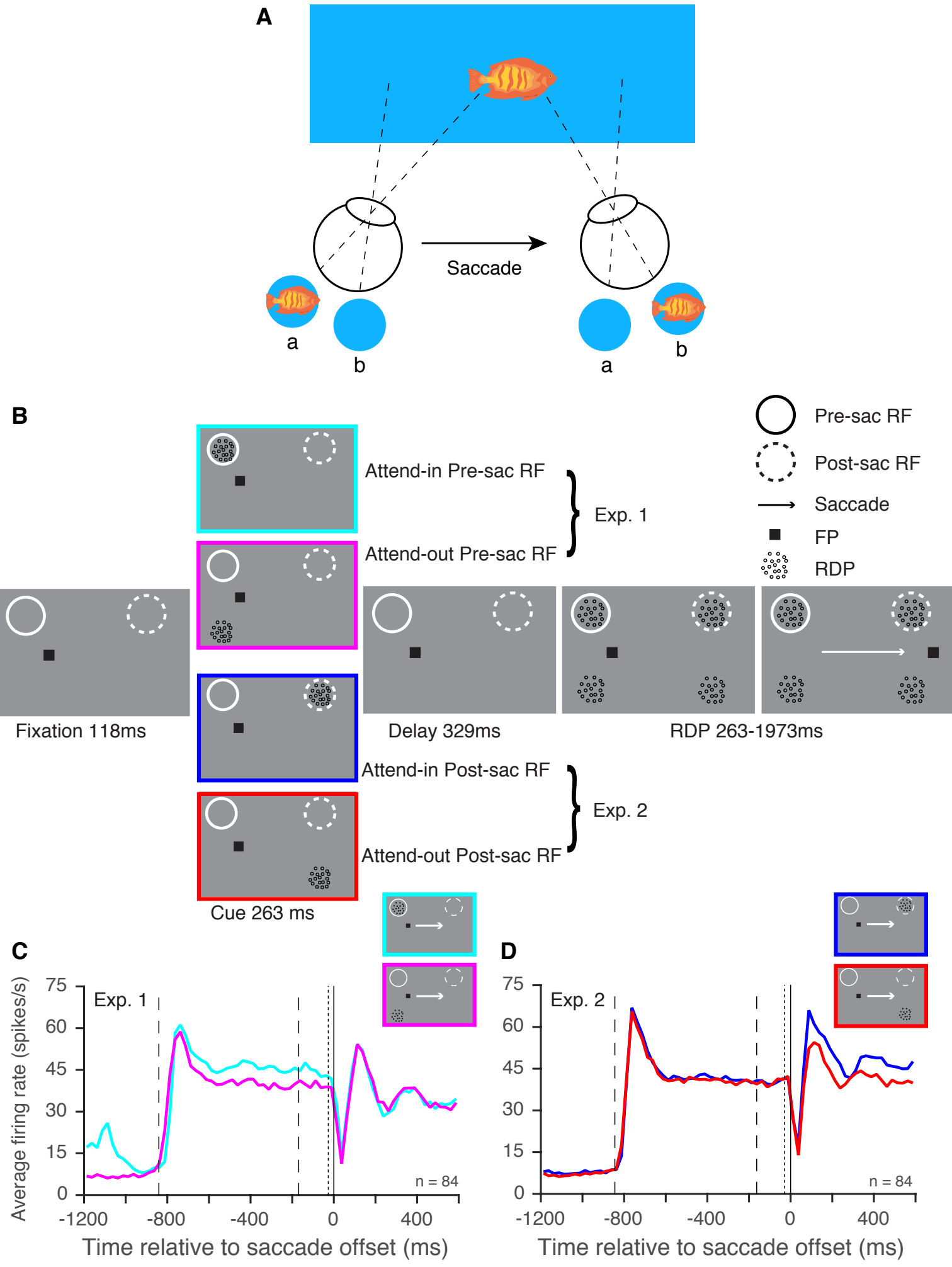
Figure 1. Attention enhances different target populations before and after a saccade. A) Cartoon demonstrating that in a retinotopic representation, an attended stimulus is represented in different populations of neurons before and after a saccade. Therefore, to maintain attention on a stimulus while making a saccade, attentional effects must manifest in different populations of neurons before and after the saccade. B) Task-design and timing: Two rhesus monkeys were trained to perform a task that involved attending to one of four moving RDPs (the target) while also making a visually guided saccade if the fixation point (FP) jumped to a new location. An initial spatial cue marked the target location on each trial. The intervening saccade changed the spatial location of the neuron's RF so that a different RDP appeared in the RF before and after the saccade. In Experiment 1, the target RDP appeared before the saccade either in the neuron's RF or at the meridionally opposite location. In Experiment 2, the same happened after the saccade. Values next to each panel represent the durations of the task phase represented by that panel. For details, see Materials and Methods. C) Population average peri-stimulus time histograms (PSTHs) in Experiment 1 aligned to the time of saccade offset. After the saccade, the stimulus in the RF was always a distractor, while before the saccade, either a target (blue curve) or a distractor (red curve) appeared in the neuron's RF: the neurons respond more in the former case before the saccade. PSTHs plotted using $25 \mathrm{~ms}$, non-overlapping bins. The inset rectangles depict the cue location for the two conditions. From left to right, the first dashed vertical line represents the mean time of RDP onset, the second dashed vertical line the mean time of fixation point jump and the dotted vertical line the mean time of saccade onset. The early response before RDP onset in the attend-in condition (blue curve) is the response to the cue. Data pooled from both monkeys. D) PSTHs for Experiment 2, in format a similar to B. Before the saccade, the stimulus in the RF was always a distractor, while after the saccade, either a target (blue curve) or a distractor (red curve) appeared in the neuron's RF: the neurons respond more in the former case after the saccade. 

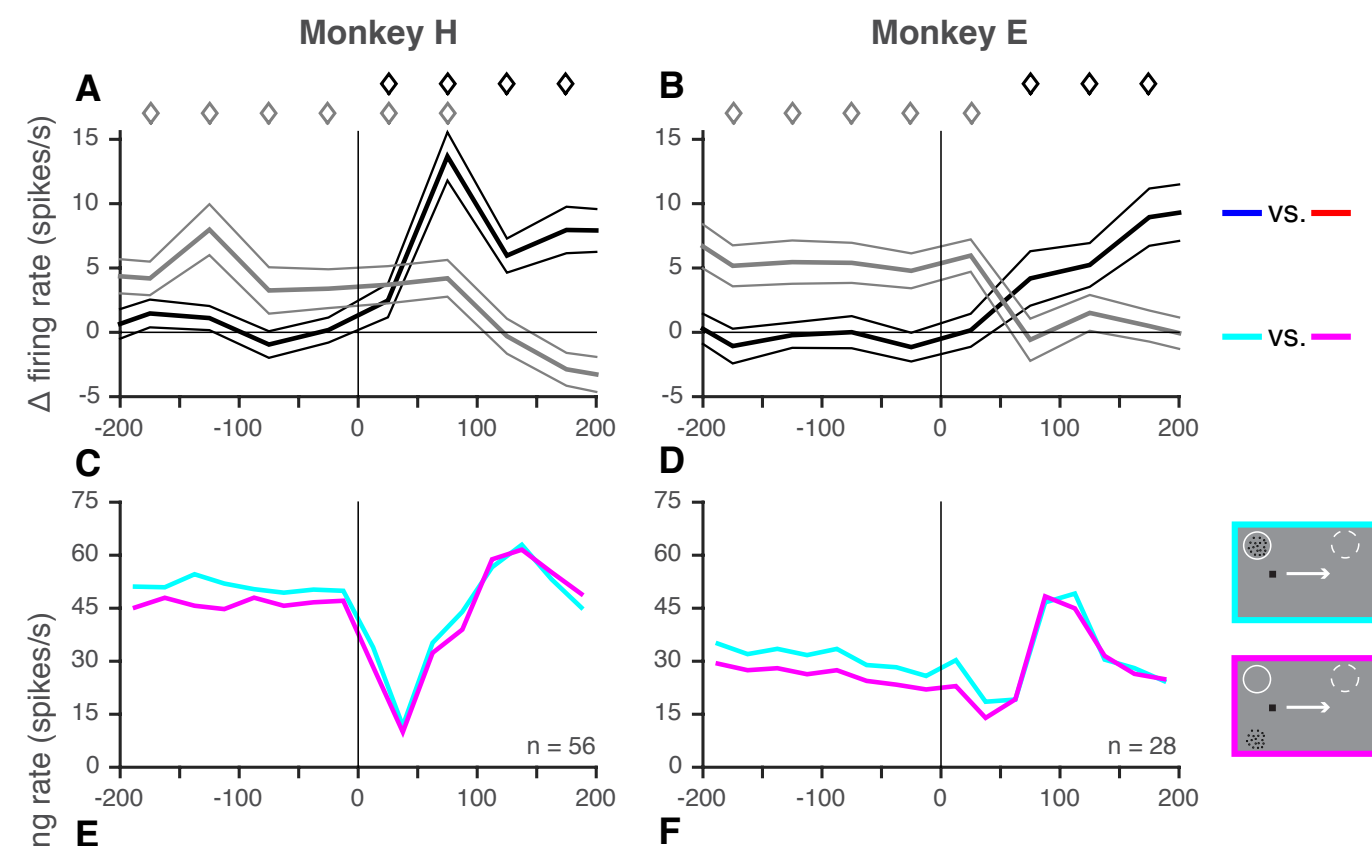

\section{D}
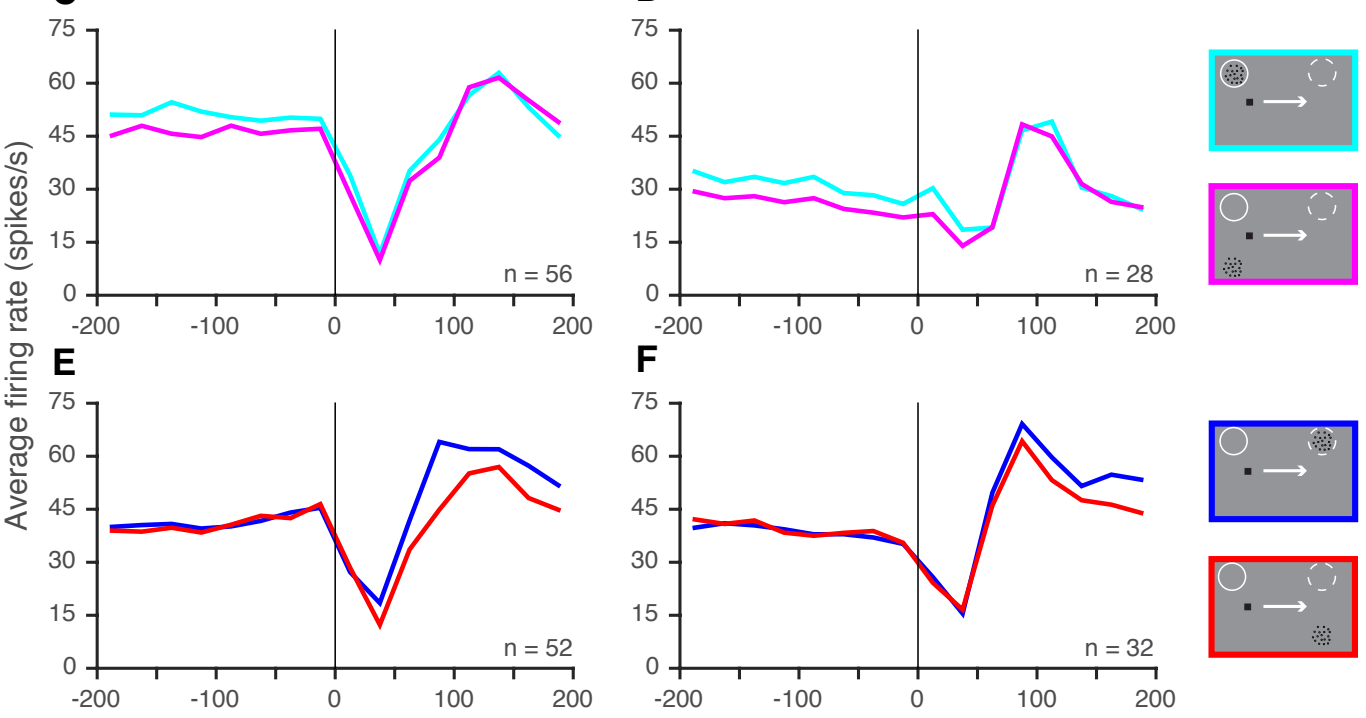

Figure 2. Attentional remapping is well-synchronized to the saccade. A,B) The attentional crossover time (when the attentional enhancement of the post-saccadic target population became larger than the attentional enhancement of the pre-saccadic target population) occurred at $31 \mathrm{~ms}(\mathrm{~A}, \mathrm{IQR}=$ $12 \mathrm{~ms}$, monkey $\mathrm{H}$ ) and $52 \mathrm{~ms}(\mathrm{~B}, \mathrm{IQR}=12 \mathrm{~ms}$, monkey $\mathrm{E})$ after saccade offset. Data for monkey $\mathrm{H}$ in $\mathrm{A}$ and for monkey $\mathrm{E}$ in $\mathrm{B}$. Grey and black curves show the mean difference (and SEMs) between the target-in-RF and distractor-in-RF curves in Experiment 1 and Experiment 2 respectively (as shown in $\mathrm{C}-\mathrm{F}$ ), but plotted using $50 \mathrm{~ms}$, non-overlapping time-bins. Diamonds above the curves indicate the successive, non-overlapping $50 \mathrm{~ms}$ time-bins in which the differences were significantly larger than zero (one-sided t-test): black diamonds for the black curve and gray diamonds for the gray curve. C-F) Same data as in Figure 1B (C-D) and Figure 1C (E-F), but plotted separately for the two monkeys and focusing on the time around the saccade (-200 to $200 \mathrm{~ms}$ relative to saccade offset); format otherwise identical. The gray curves in $A$ and $B$ are computed as the difference between the blue and red curves in $C$ and $D$ respectively, while the black curves in $A$ and $B$ are computed as the difference between the blue and red curves in $\mathrm{E}$ and $\mathrm{F}$ respectively. Also see Supplemental Figure 1. 

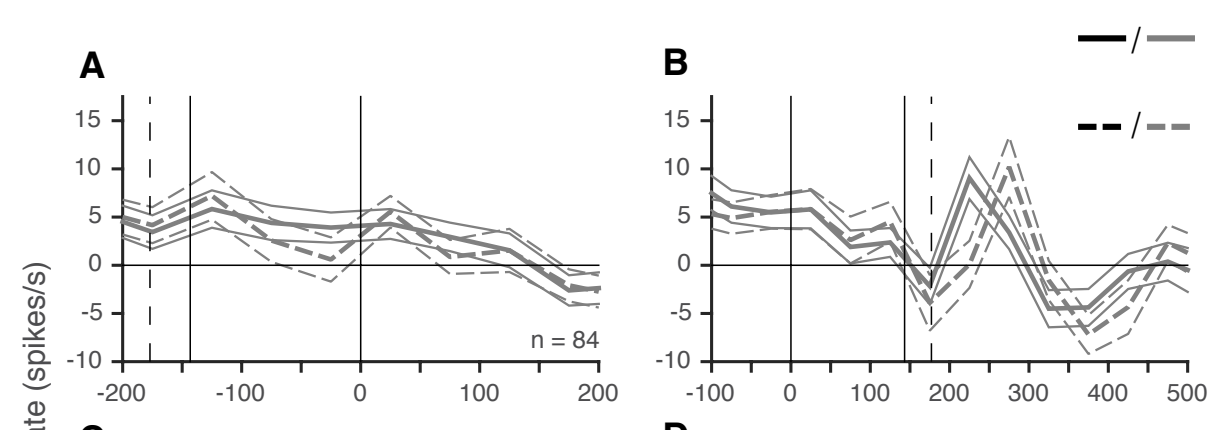

Short-latency

saccades

Long-latency
saccades

Experiment 1

D

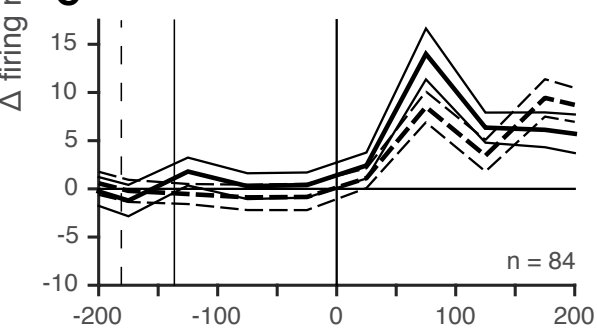

Time relative to saccade offset (ms)

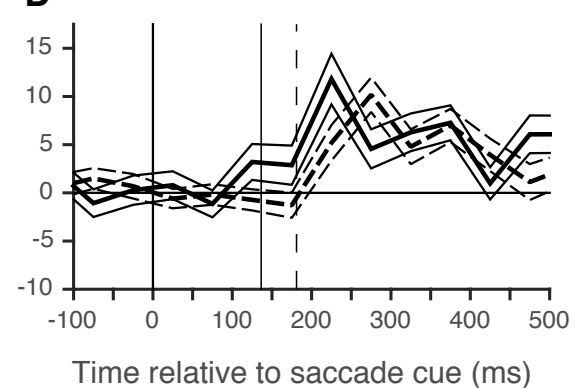

Experiment 2

Figure 3. Attentional remapping is synchronized to the saccade, not to fixation point offset. The time-course of remapping, plotted aligned to saccade offset $(A, C)$ is similar for trials with saccade latencies shorter than the 33rd percentile (solid curves) and longer than the 66th percentile (dashed curves) for the corresponding task condition, but is delayed (B,D) for the longer-latency trials by one bin $(50 \mathrm{~ms}$ ) when plotted aligned to fixation point offset: the delay was calculated as the crosscorrelation lag that yielded a maximum. The delay when aligned to saccade offset $(A, C)$ was 0 . Remapping time-course defined as in Figure 3A.B as the difference between the firing-rates in the attend-in and attend-out conditions (see Supplementary Figure 2 for the corresponding PSTHs). Results for Experiment 1 in the top row $(A, B)$ and for Experiment 2 in the bottom row (C,D). Solid and dashed vertical lines in each panel represent the mean time of fixation-point offset $(A, C)$ or saccade latency $(B, D)$ for trials with short-latency and long-latency saccades respectively. Data from both monkeys were pooled for this analysis (see Text and Supplemental Figures 2 and 3). 
Monkey E
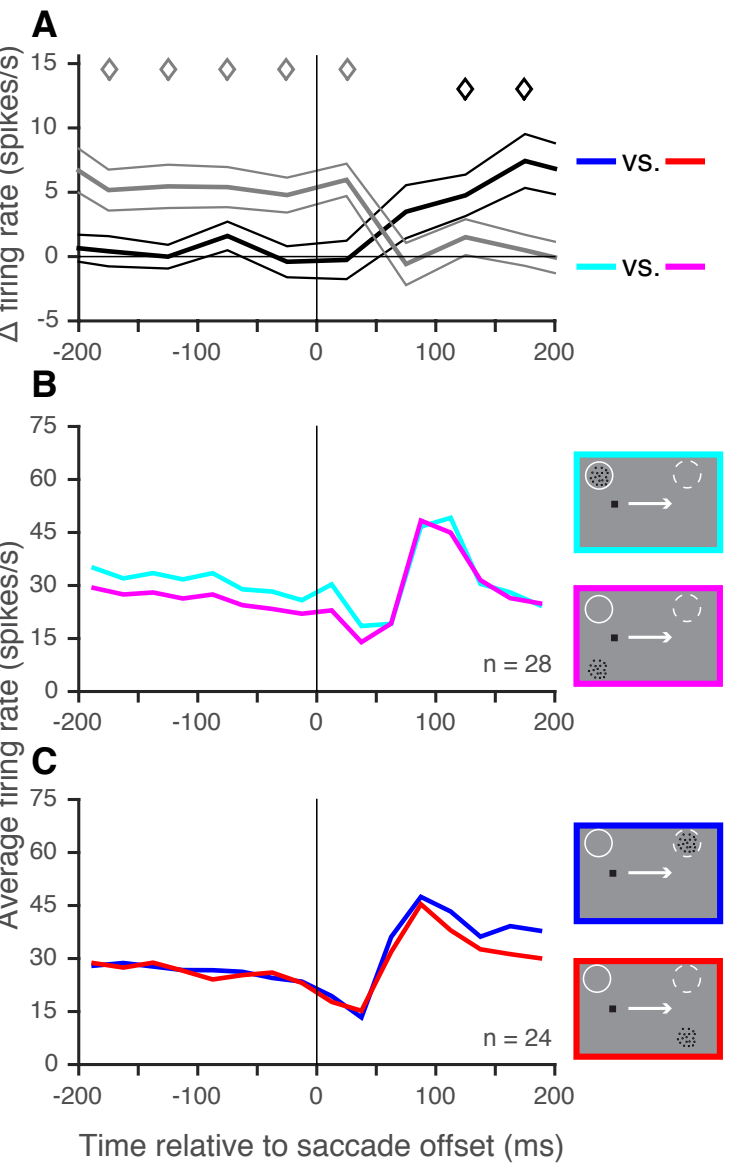

Supplemental Figure 1. Attentional remapping remains well-synchronized when the firingrates are matched between the populations in Experiment 1 and 2 in monkey E. Related to Figure 2. Figure format identical to Figure 2, except that only data from monkey $E$ are shown, and the $25 \%$ of highest-firing rate neurons from Experiment 2 were excluded from the sample. The attentional cross-over time now occurred at a median time of $56 \mathrm{~ms}$ (IQR =13 ms) after saccade offset (compared to $52 \mathrm{~ms}, \mathrm{IQR}=12 \mathrm{~ms}$ in the full sample in Figure 2B). 
A

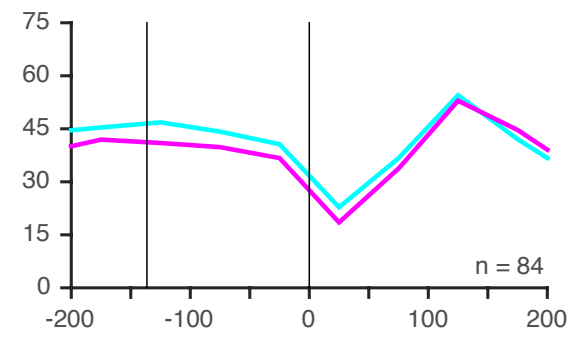

C

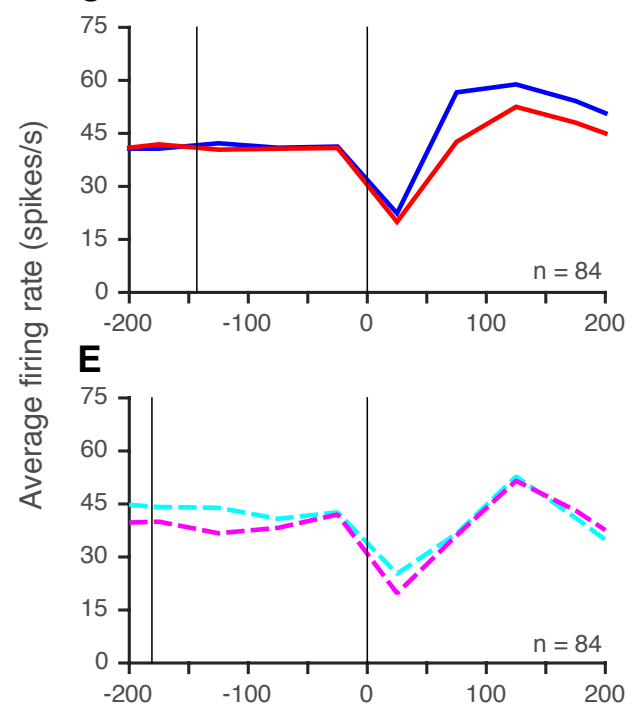

G

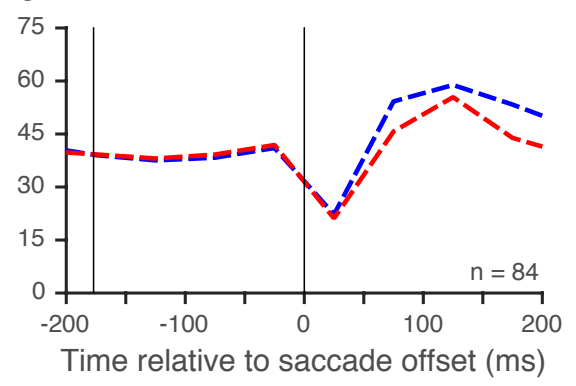

B
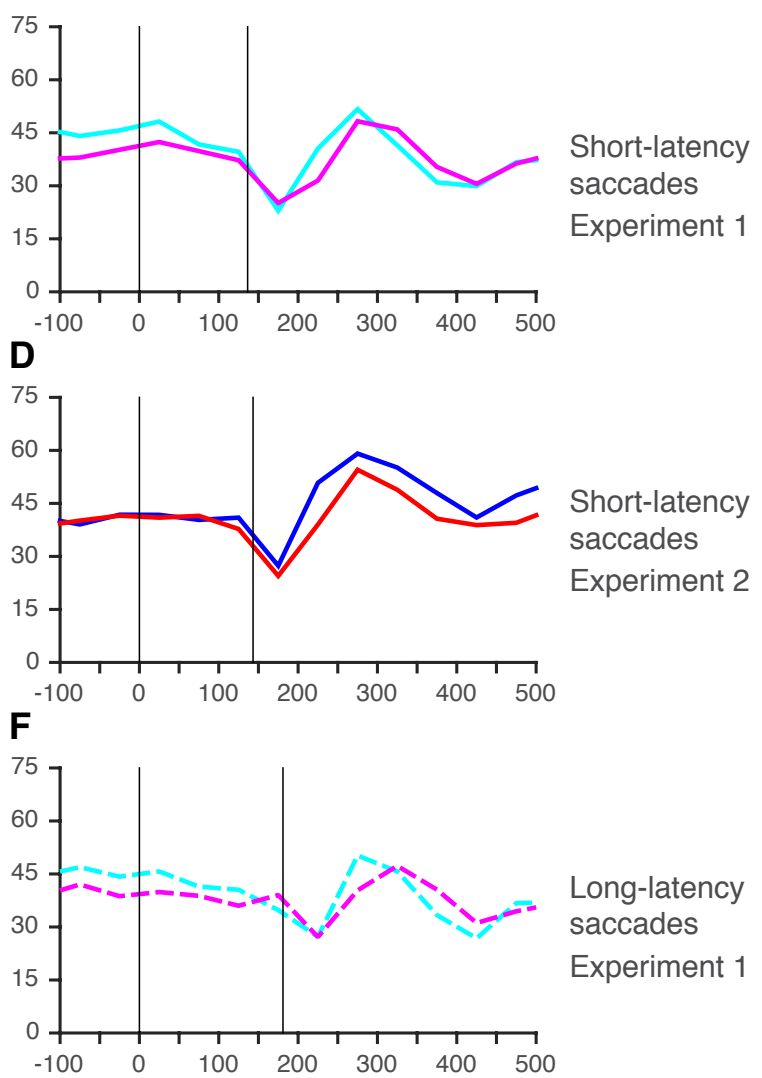

H

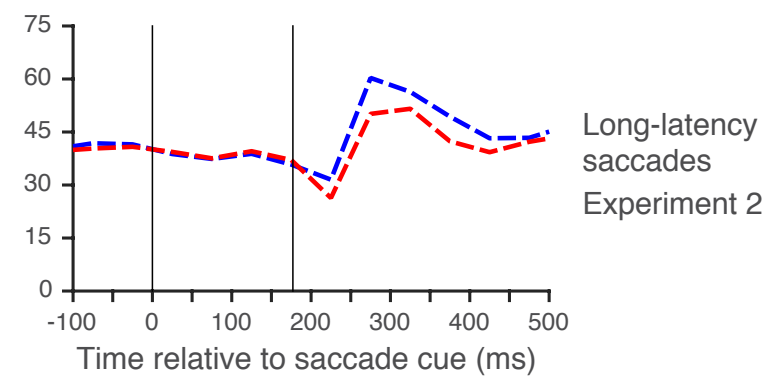

Supplemental Figure 2. The time-course of attentional remapping is similar when aligned to saccade offset, not fixation point offset. Related to Figure 3. PSTHs from which the difference curves in Figure 3A-D were derived. As in Figure 2, the difference curves use $50 \mathrm{~ms}$ non-overlapping time-bins, while the PSTHs use $25 \mathrm{~ms}$ non-overlapping time-bins. Data plotted for trials with saccade latencies shorter than the 33rd percentile (A-D) and longer than the 66th percentile $(E-H)$ for the corresponding task conditions in Experiment 1 ( $A, B, E, F)$ and Experiment $2(C, D, G, H)$. Left column shows data aligned to saccade offset and the right column shows data aligned to fixation point offset. Vertical lines away from 0 indicate the mean time of fixation point offset (left column) and the mean saccade latency (right column). Data from both monkeys were pooled for this analysis (see Methods). 

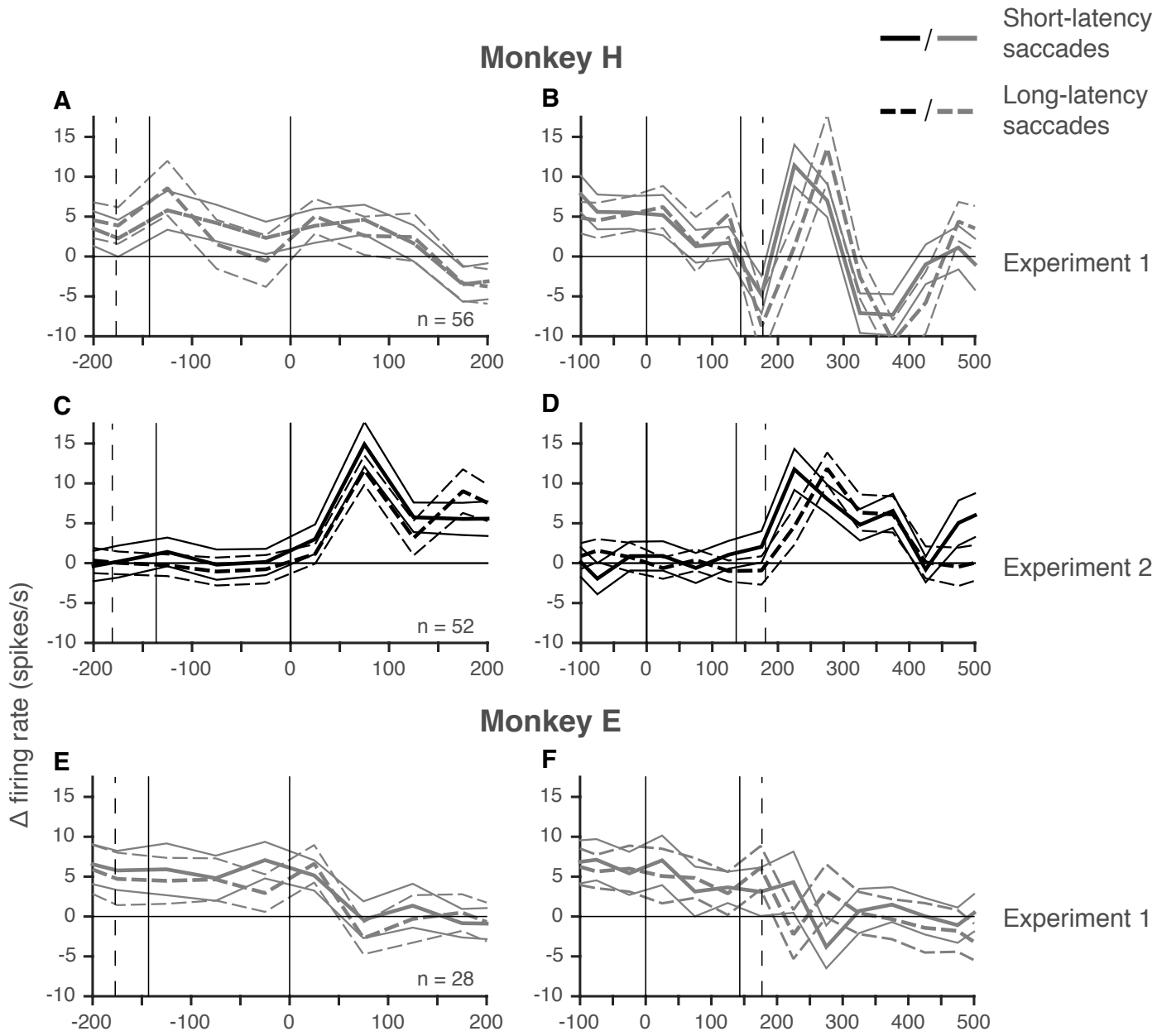

D

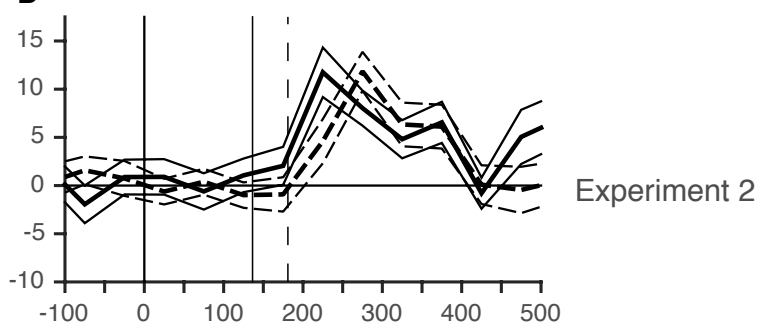

Monkey E

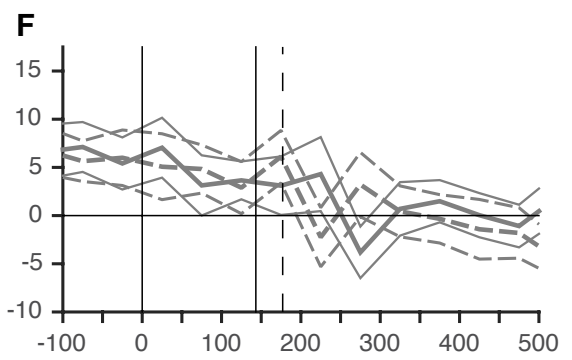

Experiment 1

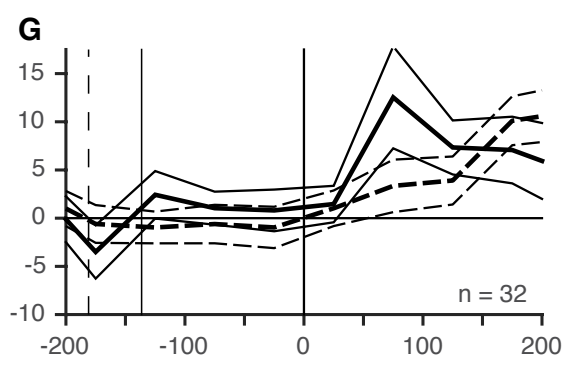

Time relative to saccade offset $(\mathrm{ms})$

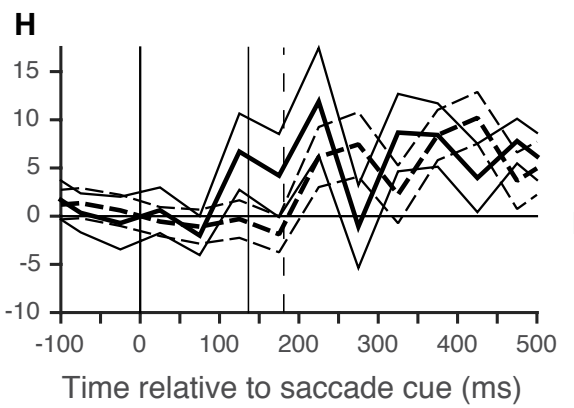

Experiment 2

Supplemental Figure 3. The patterns in the pooled data of Figure 3 are generally consistent with those in the individual monkeys. Related to Figure 3. Figure format identical to Figure 3, except that instead of the pooled data in Figure 3, data are shown separately for monkey H (A-D) and monkey $E(E-H)$. The remapping time-course remained superimposed when aligned to saccade offset for both monkeys (A,C, E and G; estimated delay $=0 \mathrm{~ms}$ in all cases). When aligned to fixation point offset, the time-course of attentional enhancement of the post-saccadic population (in Experiment 2) in both monkeys showed the same delay pattern for long-latency saccade trials seen in the pooled data (estimated delay $=50 \mathrm{~ms} ; \mathrm{D}, \mathrm{H}$ ), as expected if attentional remapping was co-ordinated with saccade planning/execution. The time-course of attentional decay in the pre-saccadic population was more variable $(\mathrm{C}, \mathrm{G})$. Similar to the pooled data, monkey $\mathrm{H}$ showed superimposed curves at early times and a $50 \mathrm{~ms}$ delay later (from $200 \mathrm{~ms}$ after fixation point offset; C). However, monkey E did not show this delay: the attentional decay in the pre-saccadic population even appeared to be earlier (with a peak lead of $50 \mathrm{~ms}$ ) in trials with longer-latency saccades (F). 
Additional supplementary materials 1: Example eye traces

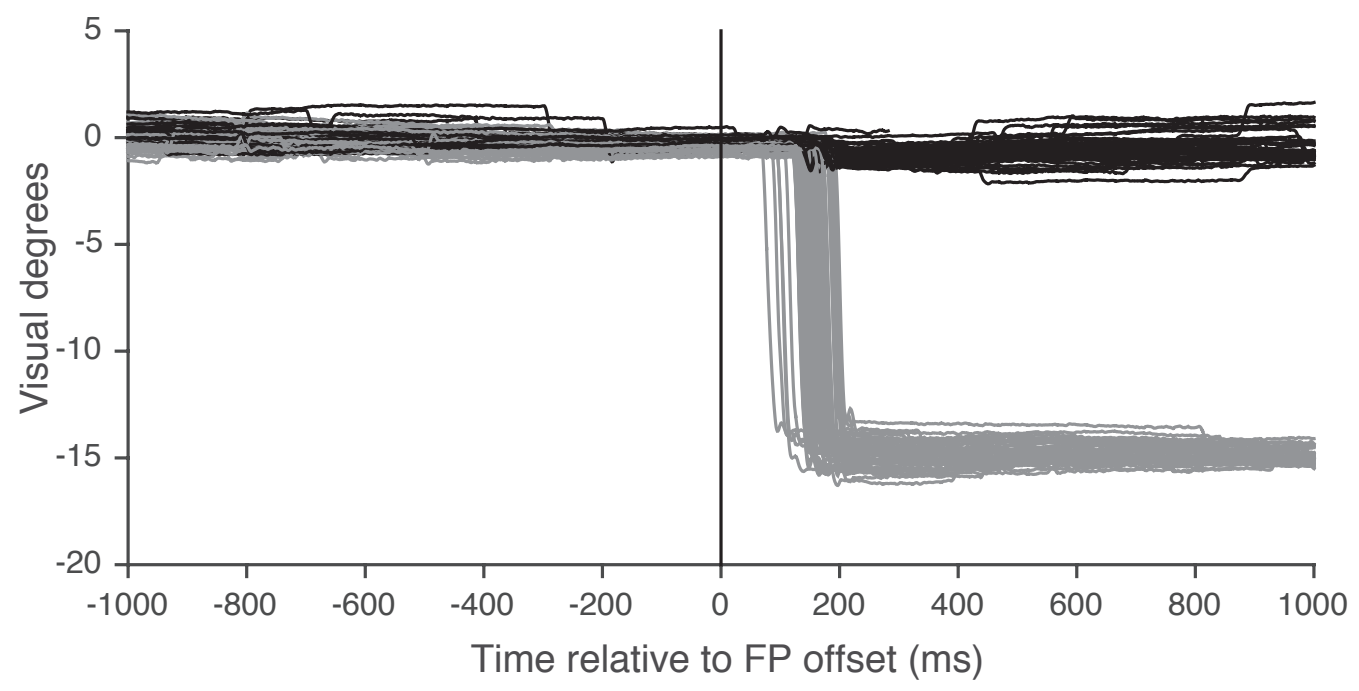

The example eye traces of trial No. 80-180 from Monkey E in the experiment 1 when he was performing the task and made a sacccade in that trial in an example session. The black and gray traces indicate the horizontal and vertical eye position respectively. In this session, the animal was required to perform a 15 visual angle degrees saccade to the upwards when he saw the fixation point jump. (recording session: tao-cueRF-edg-036-001+02). 


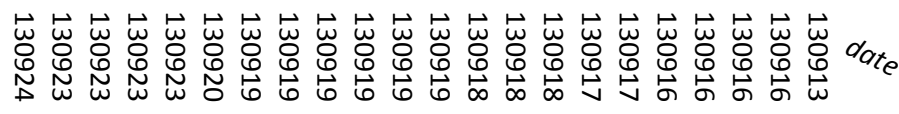

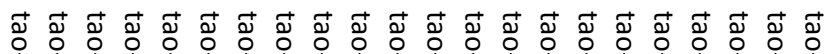

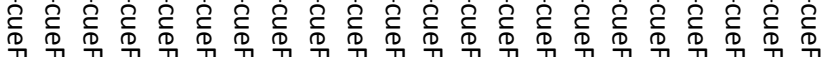

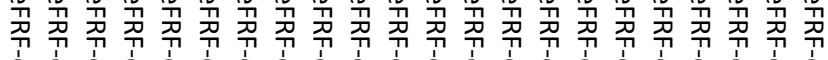
然

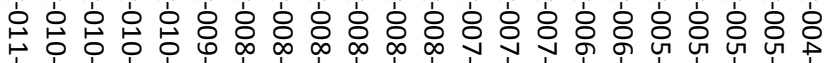

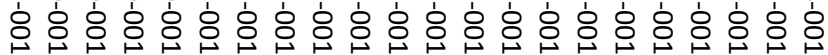

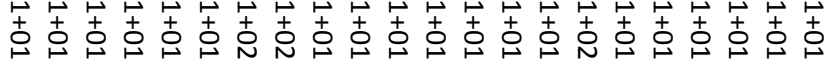

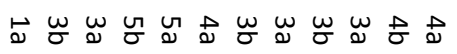

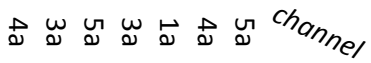

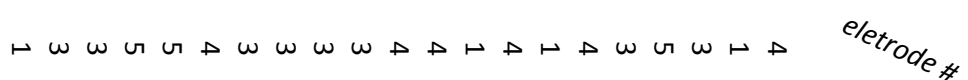

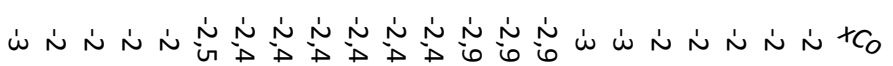

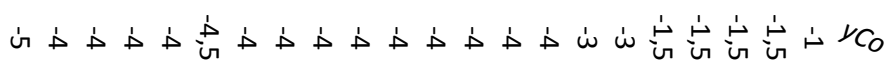

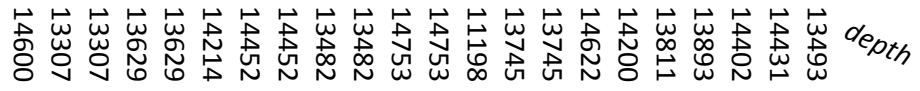

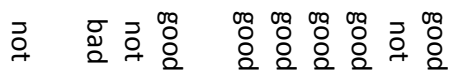

$\infty \infty \infty \infty \bullet \infty \omega \infty \infty \infty \infty$

000000000000

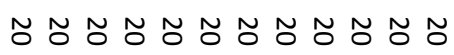

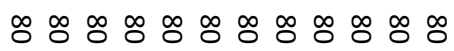

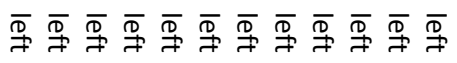
aVVVV•

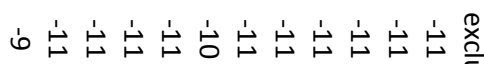

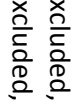

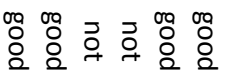
$\infty \infty \infty \infty \infty 6$ 000000

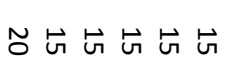

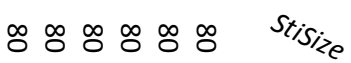

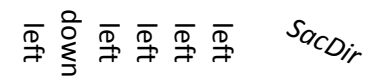

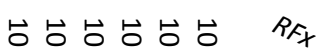
齐它的它它它它 P P

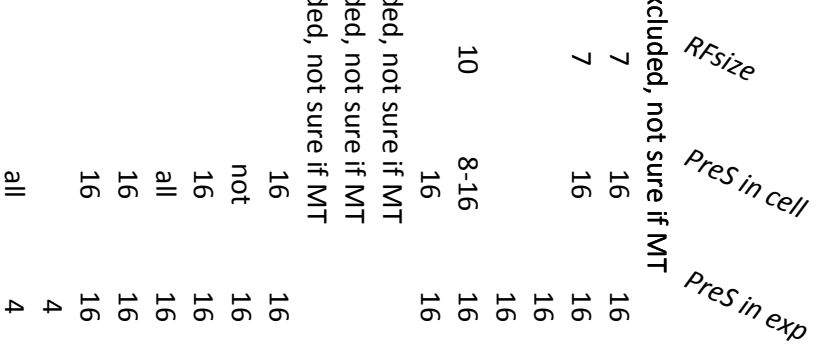

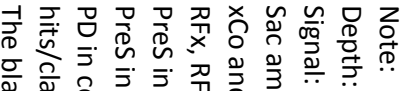

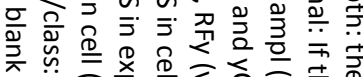

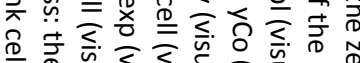

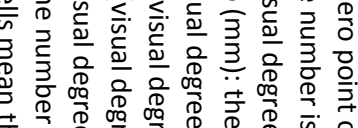

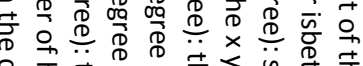

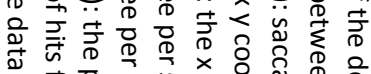

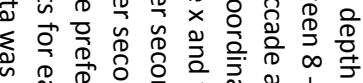

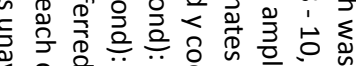

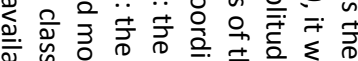

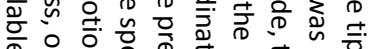

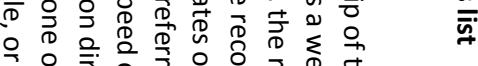

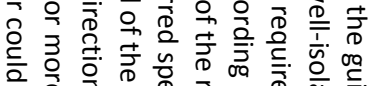

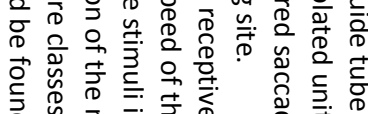

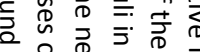

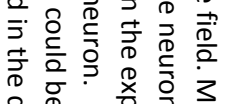

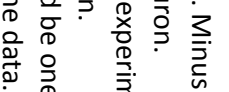
章

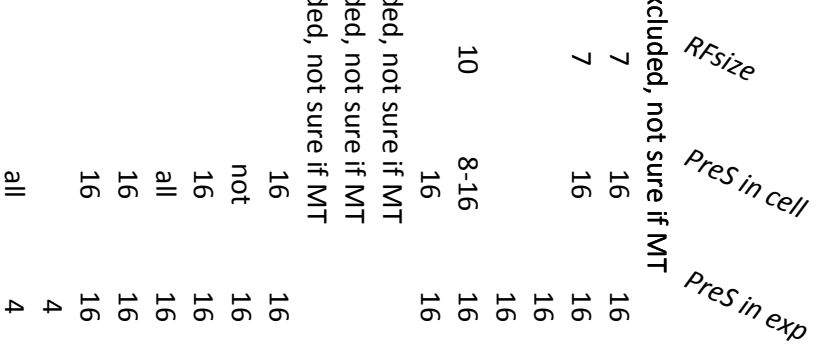

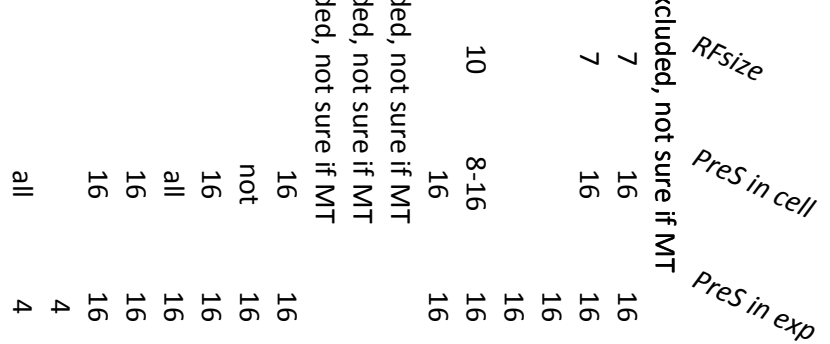

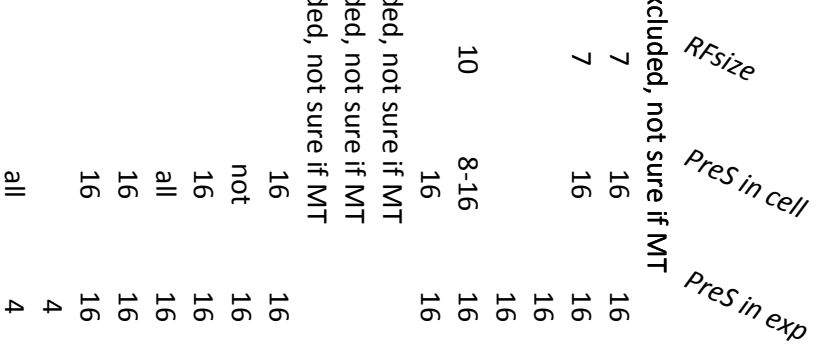

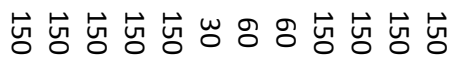
๖

$$
\begin{aligned}
& D_{D_{i n}} \\
& \text { lagres }_{e_{p}}
\end{aligned}
$$

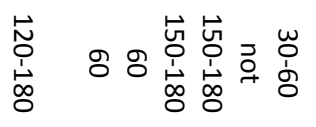

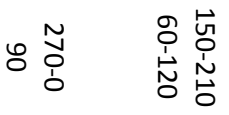
os in cell

ஜ용 \&

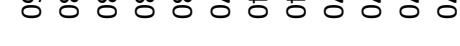

용항 뭉ㅁㅇ

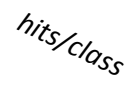




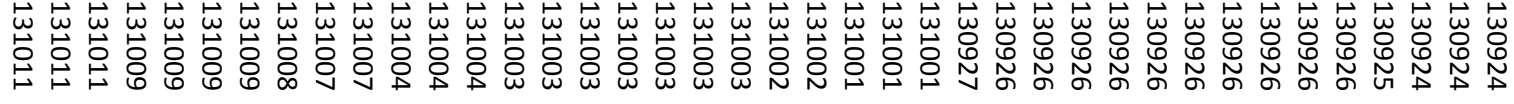

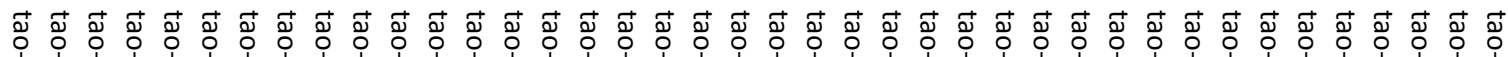

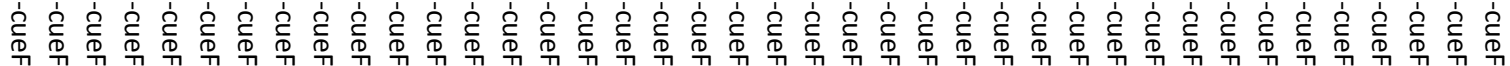

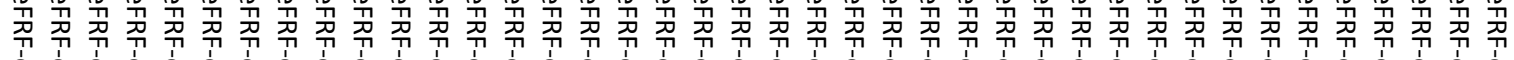

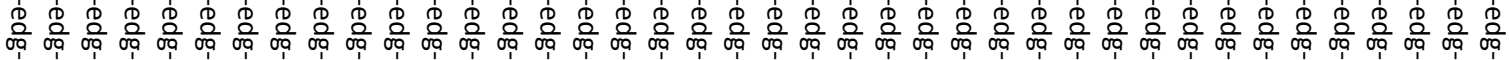

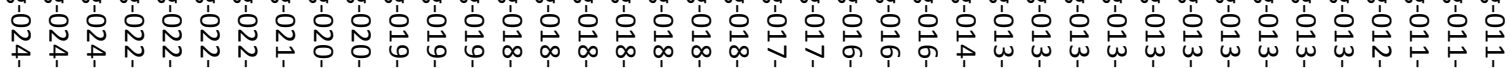

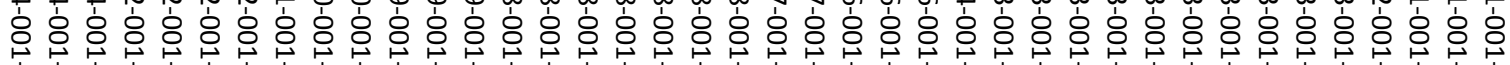

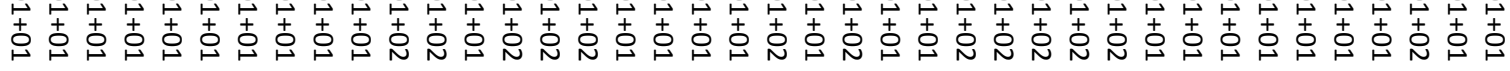

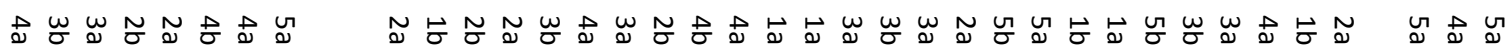

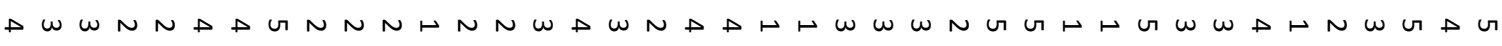

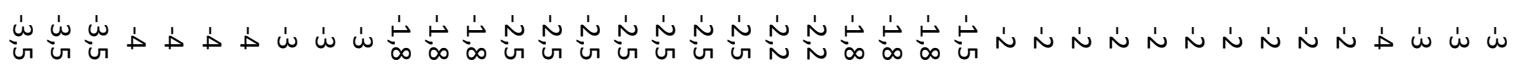

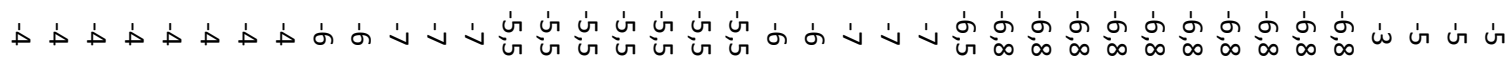

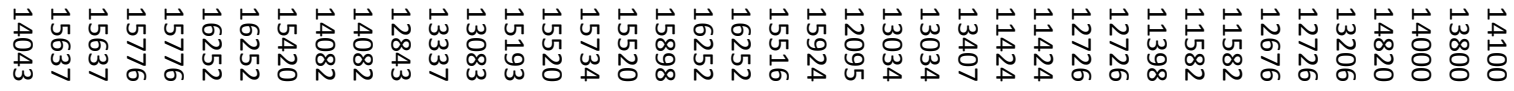

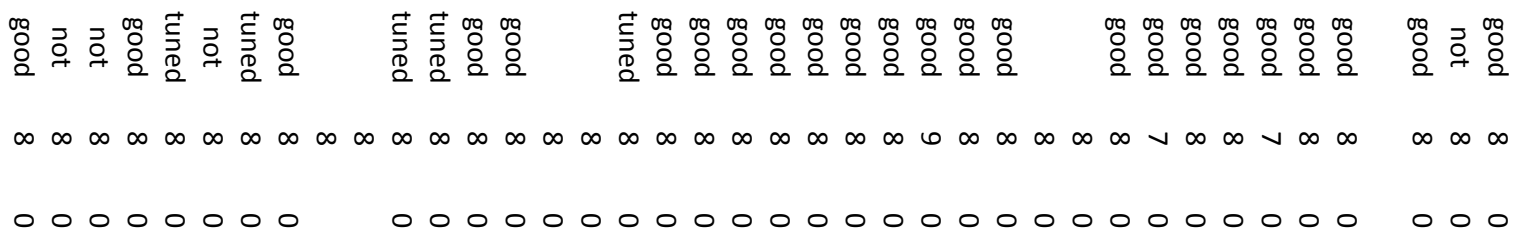

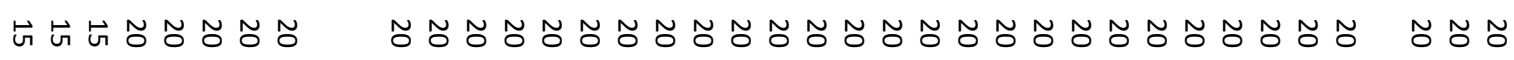

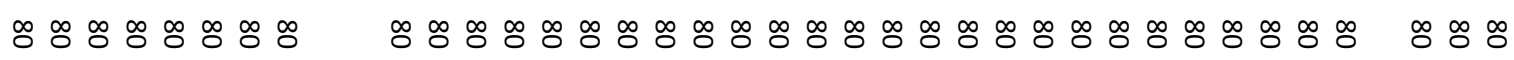

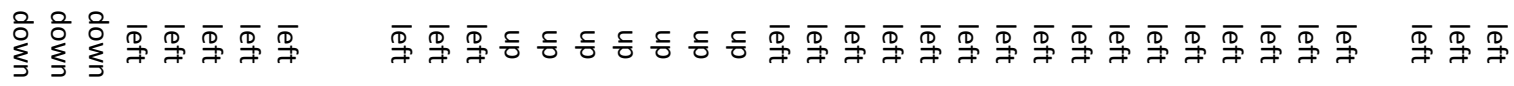
०

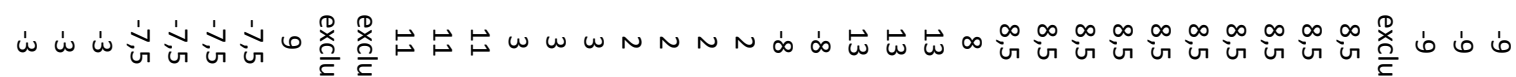
Vv

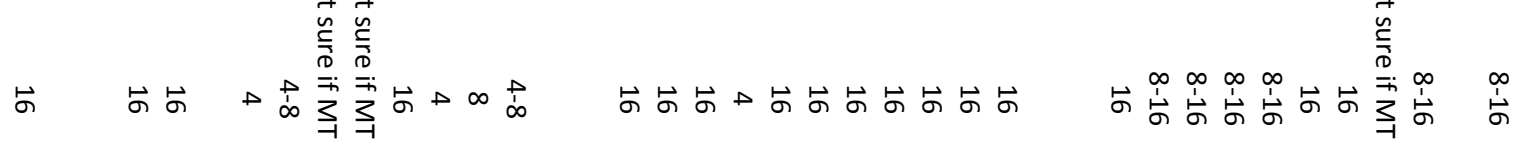

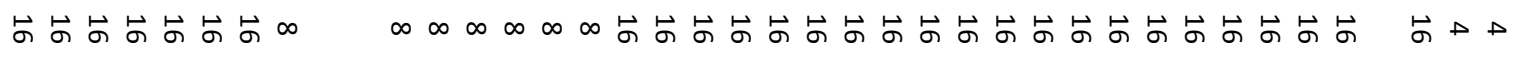

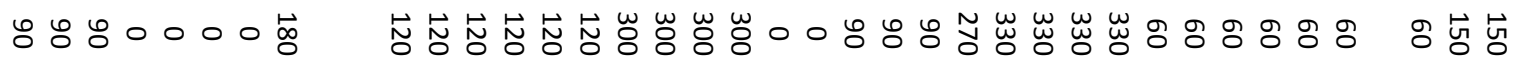

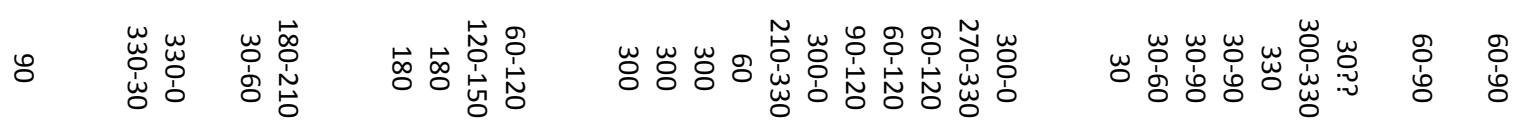

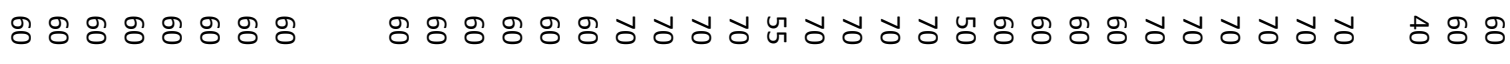




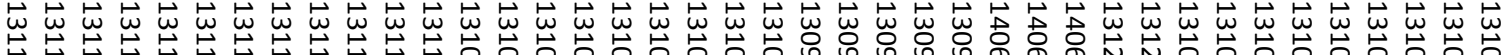

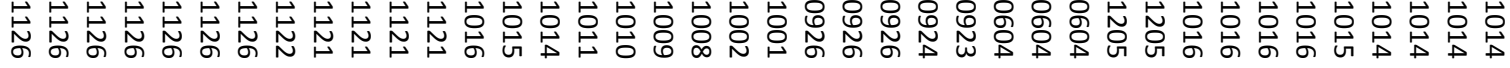

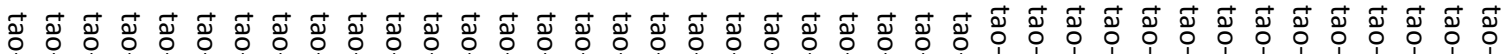

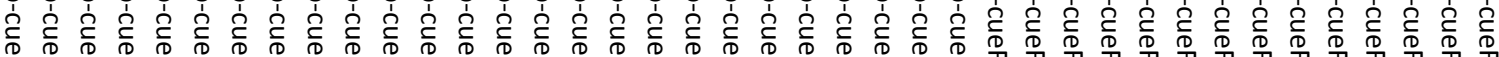

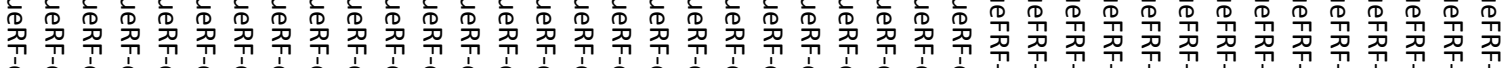

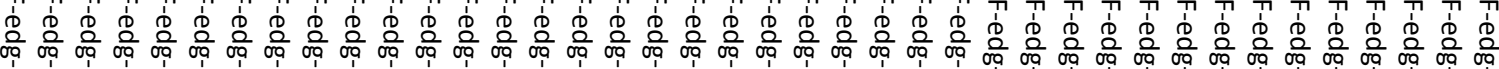

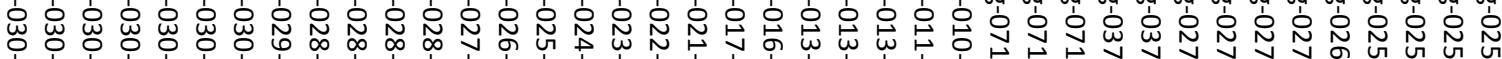

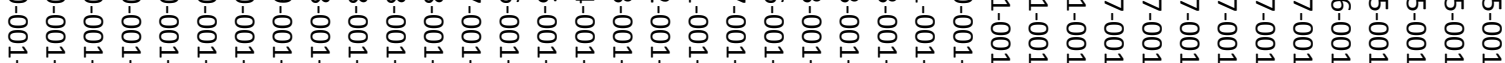

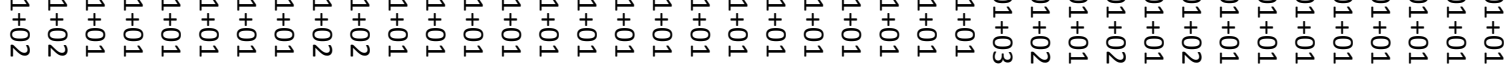

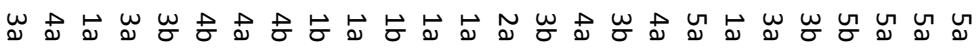
ษ $\widetilde{\sigma} \circlearrowleft$

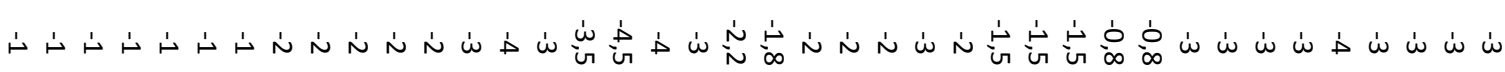

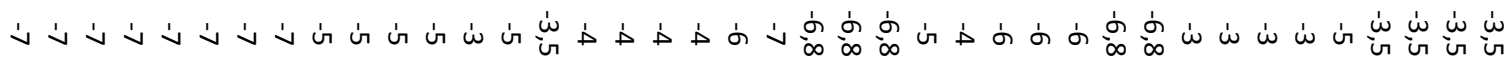

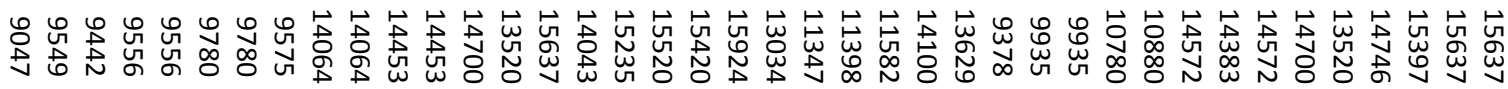

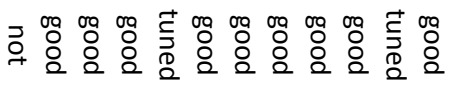

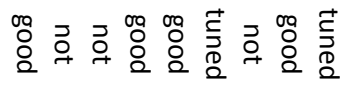

$\infty \quad \infty \quad \infty \quad \infty \quad \infty \quad \infty \quad \infty \quad \infty \vee \infty \infty$

ํ.

000000000000

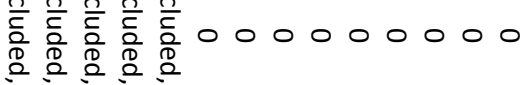

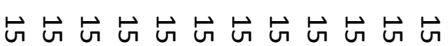

œ

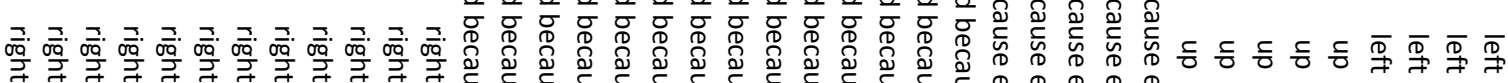

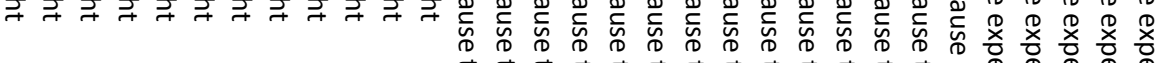

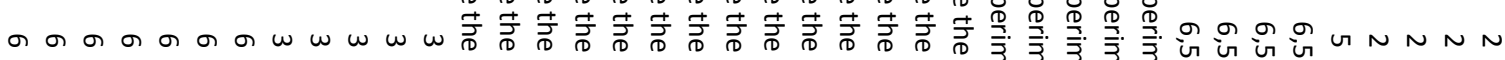

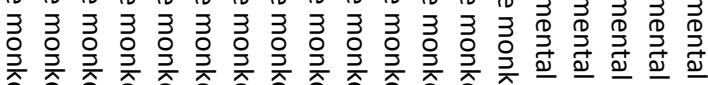

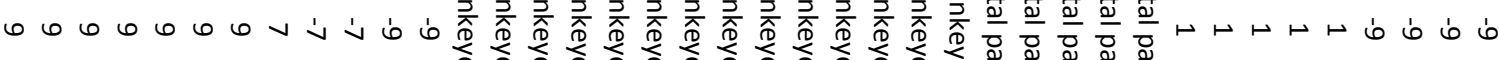

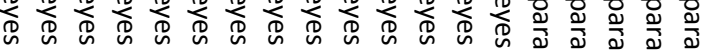

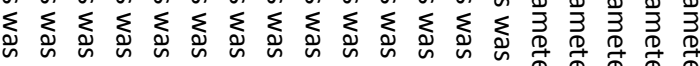

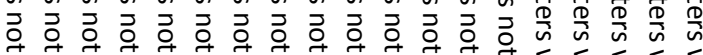

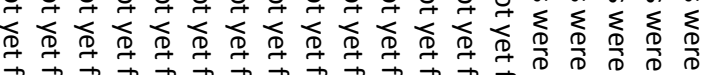

ウே

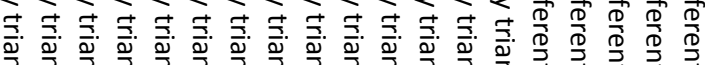

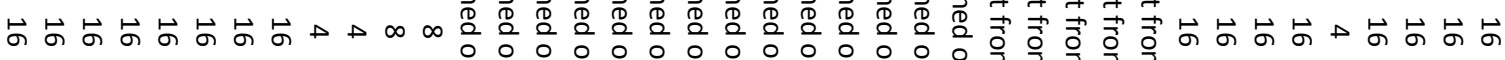

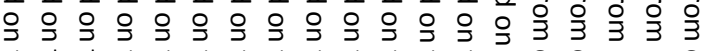

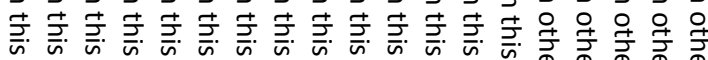

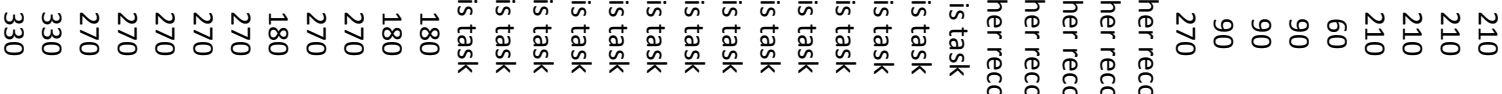

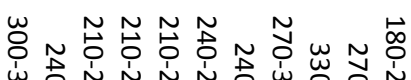

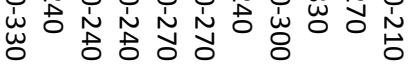

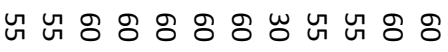

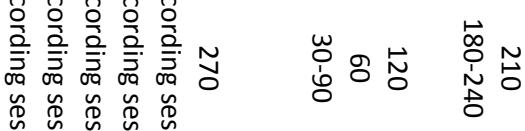

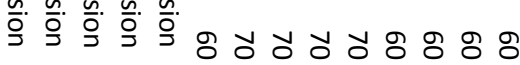


岕

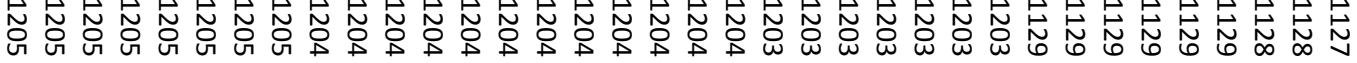

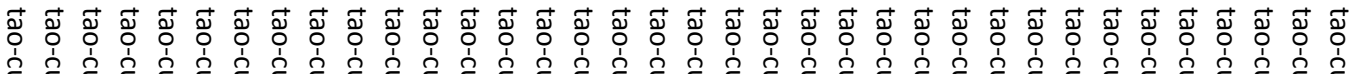

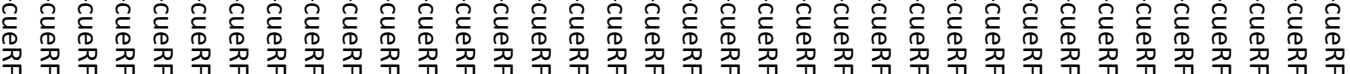

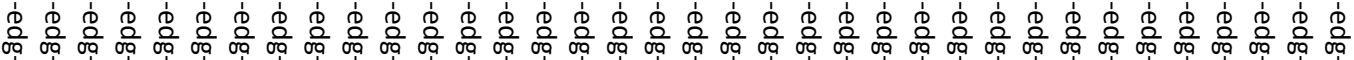

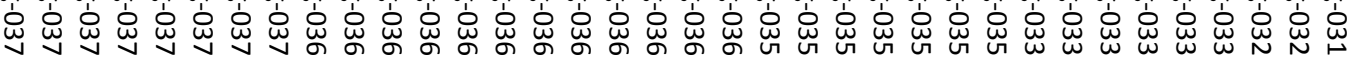

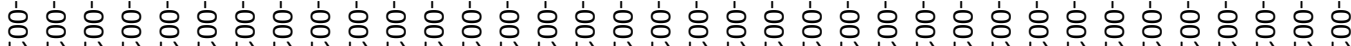

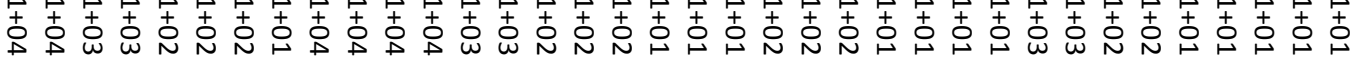

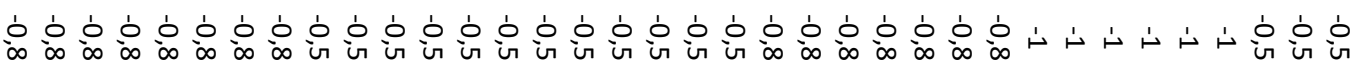

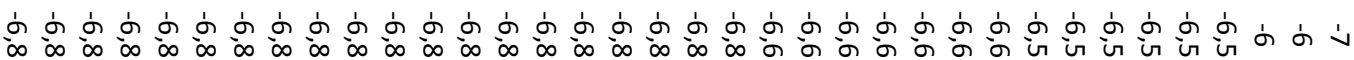

เ

$\infty$

$\frac{\text { ㅁ. }}{2}$

6

•

மே

மே

$\infty \quad \infty \quad \infty \quad \infty \infty \infty \infty \infty \infty \infty \infty \infty \infty \infty \infty$

000000000000000

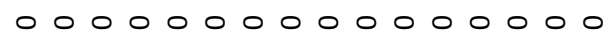

ㅂ เ

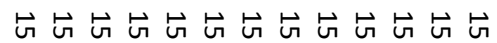

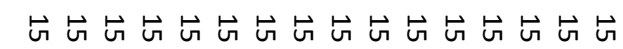

車

등 등

등 등 등 등 등 등 등 등 등 등 등 등 등

๖っ

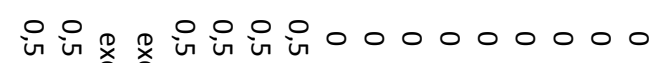

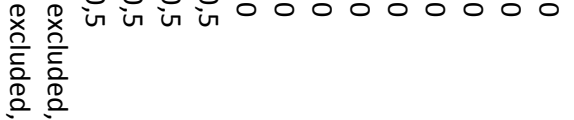

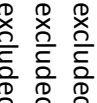

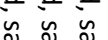

o

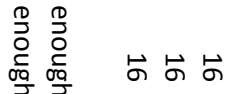

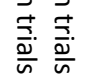

穴灾

స్ర in

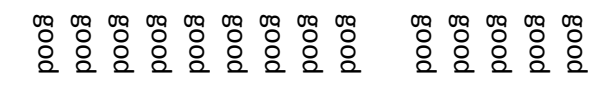

$\infty \infty \infty \infty \infty \infty \infty \infty \infty \infty \infty \infty \infty \infty \infty$

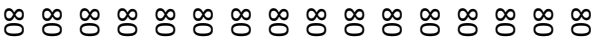

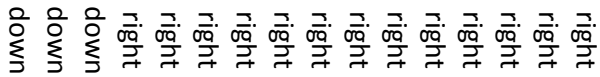

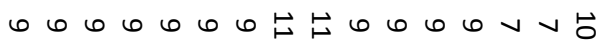

w

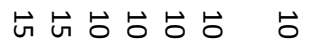

の

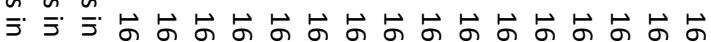
志 志 总

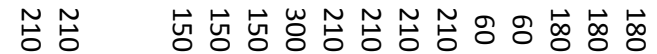

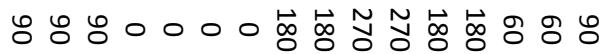

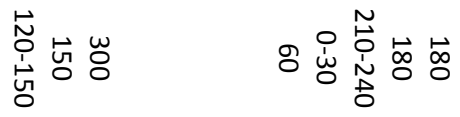

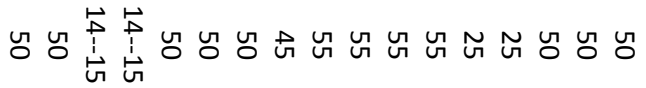

:

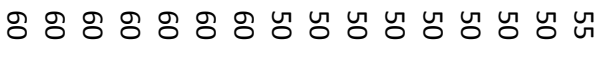




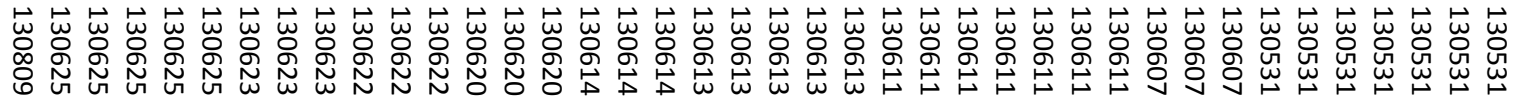

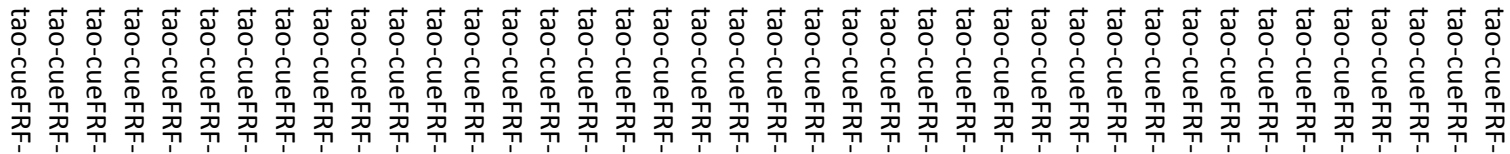

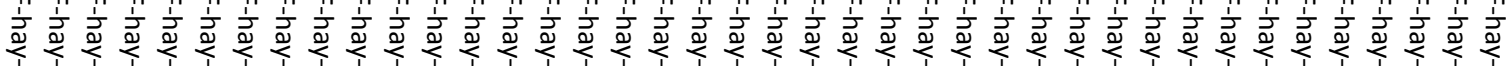

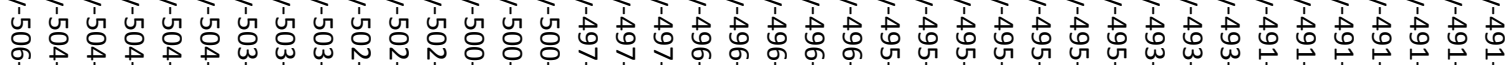

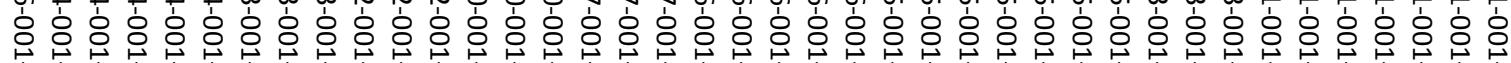

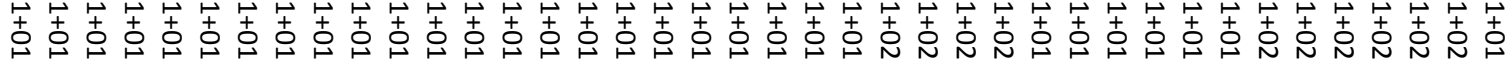

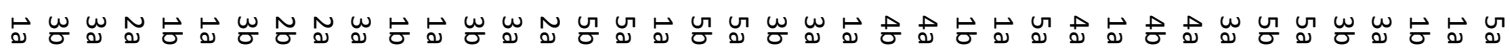

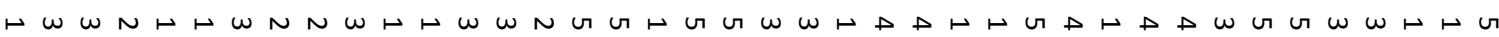

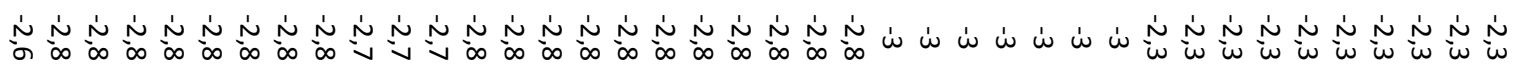

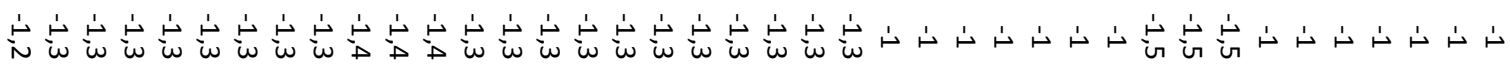

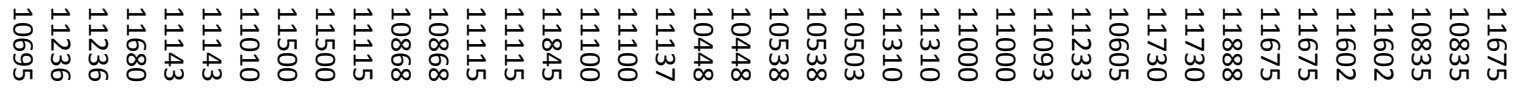

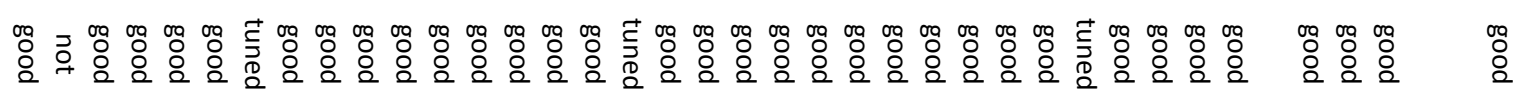

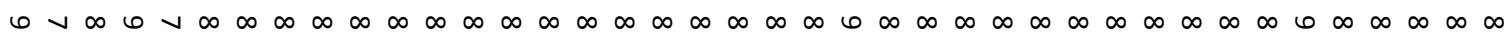
0000000000000000000000000000000000000000

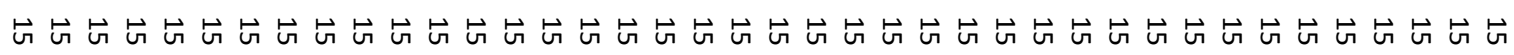

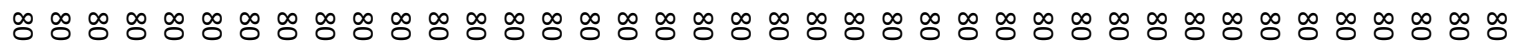

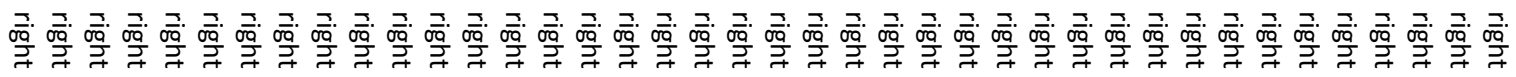

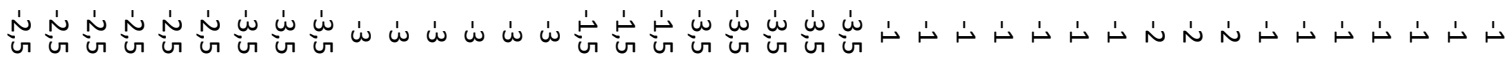

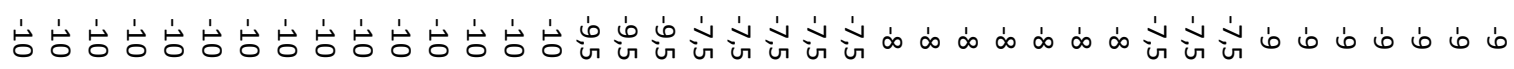

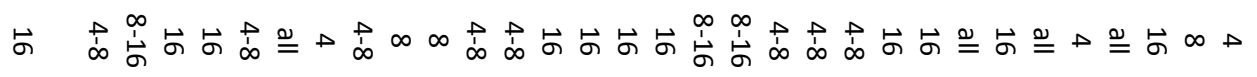
兽

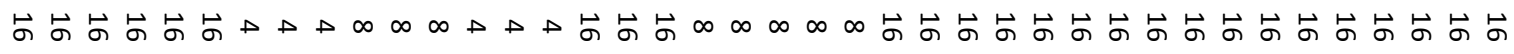

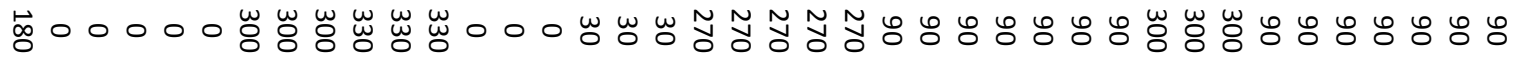

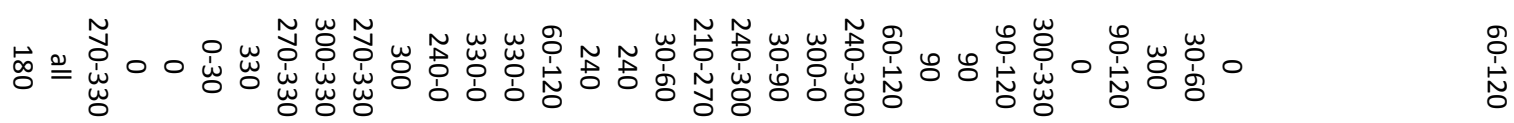

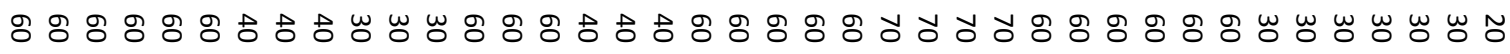




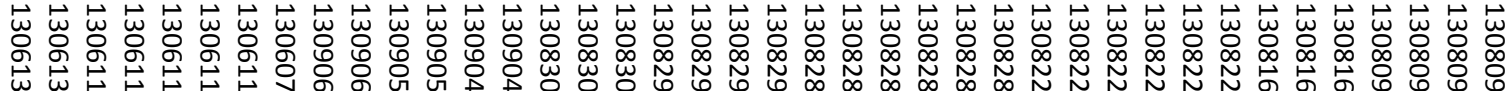

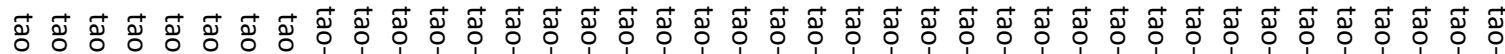

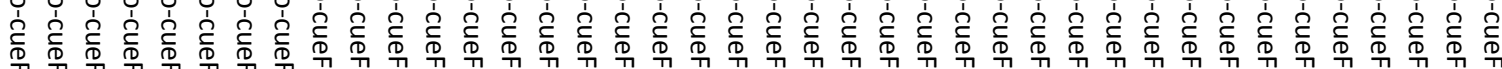

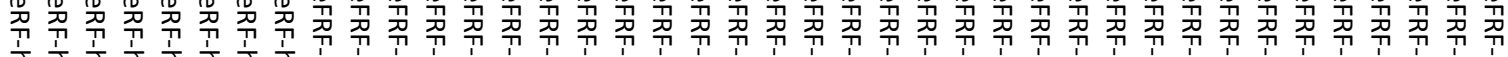

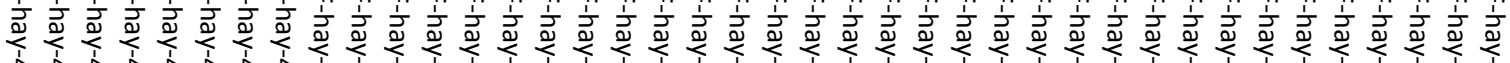
to

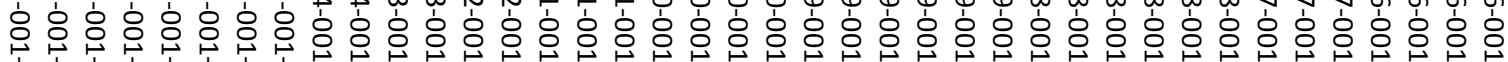

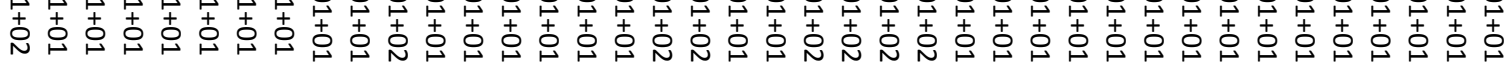

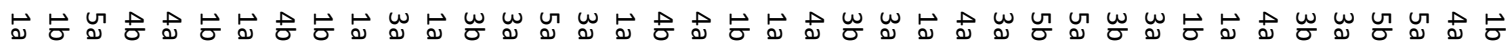

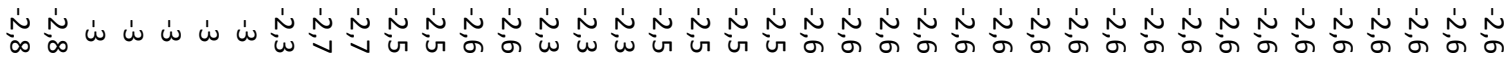

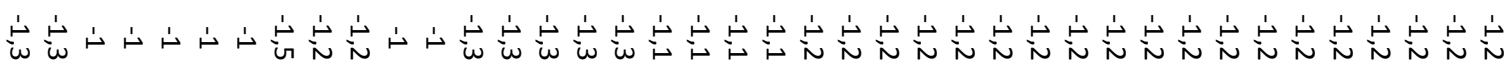

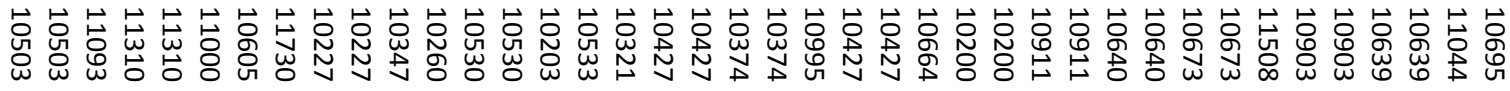

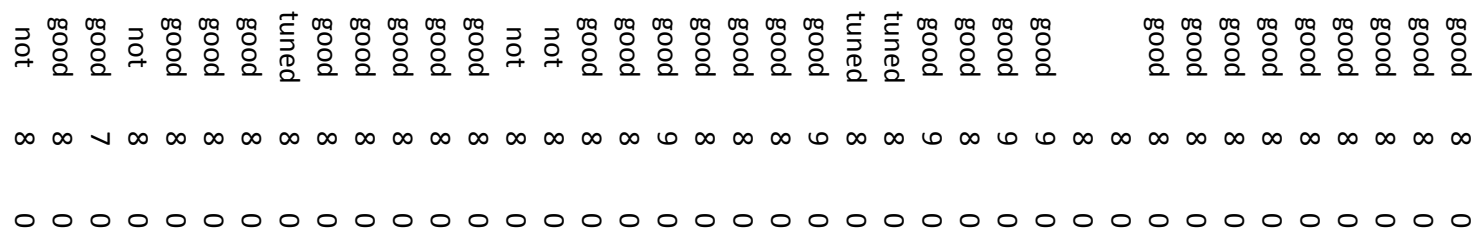

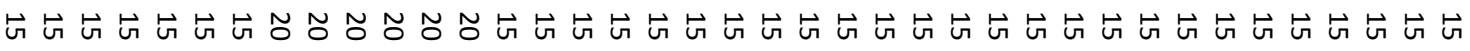

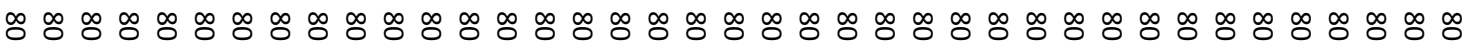

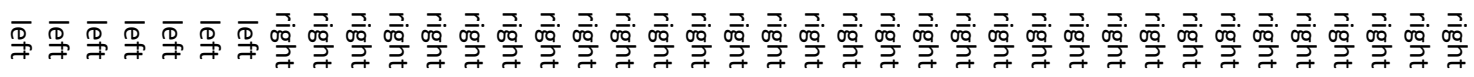

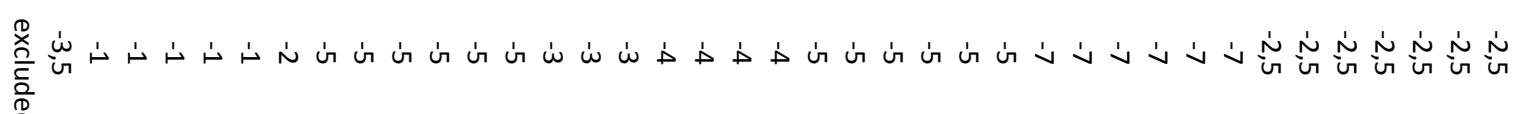

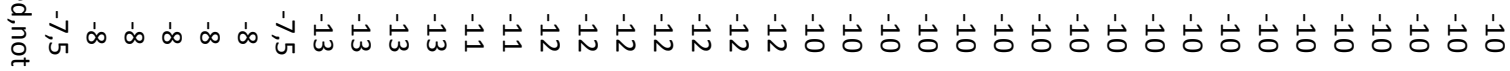

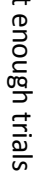

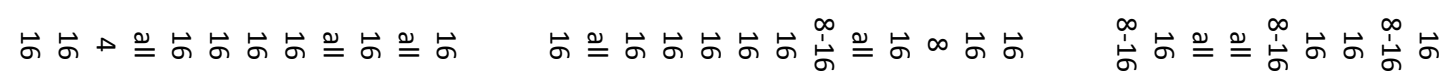

め 大

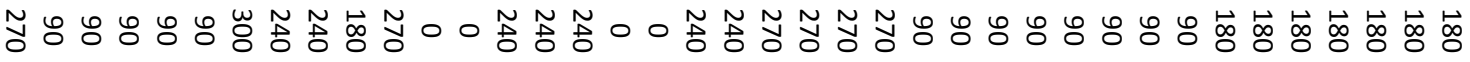

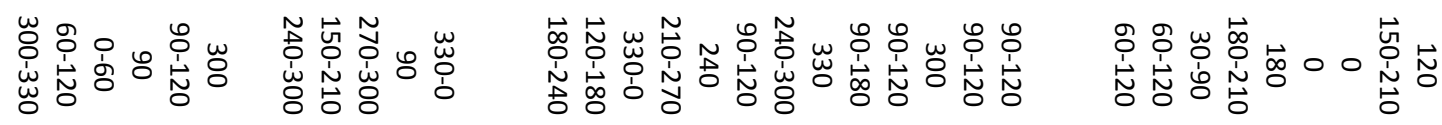

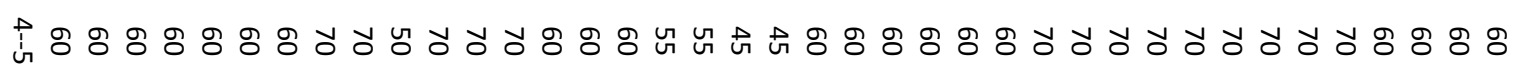




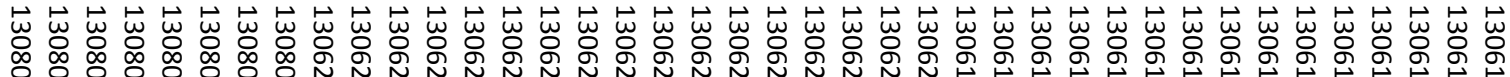

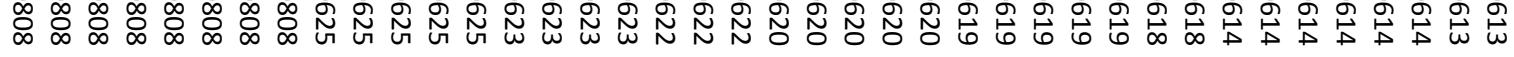

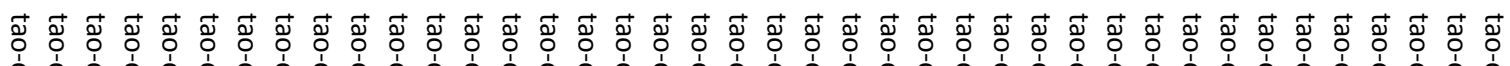

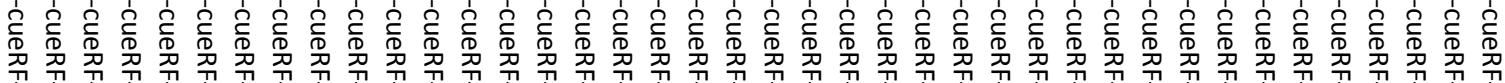

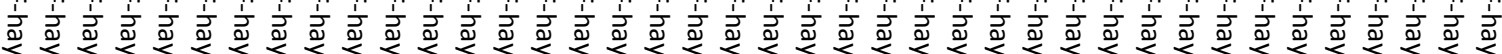

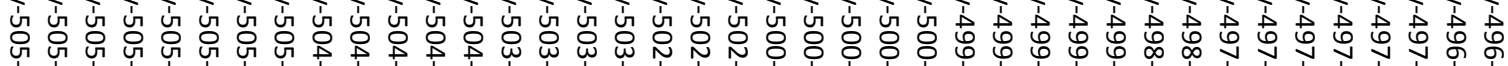

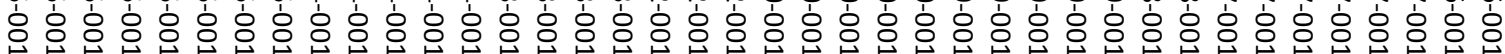

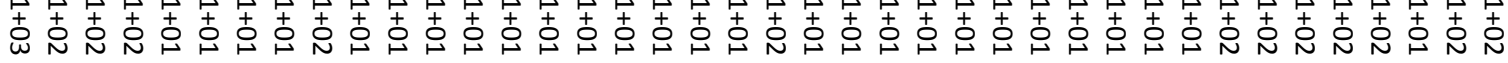

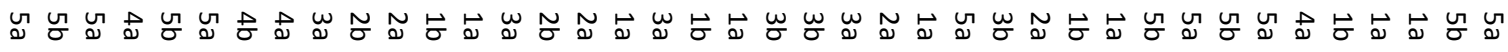

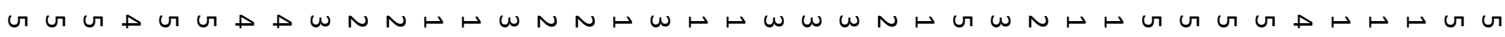

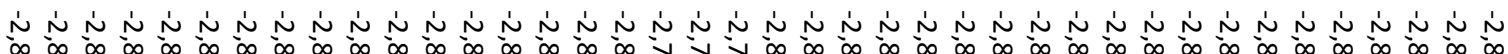

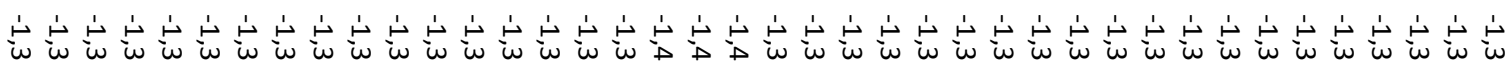

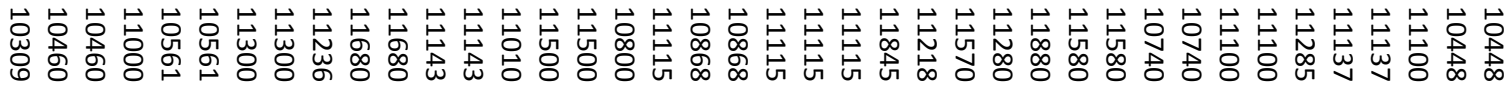

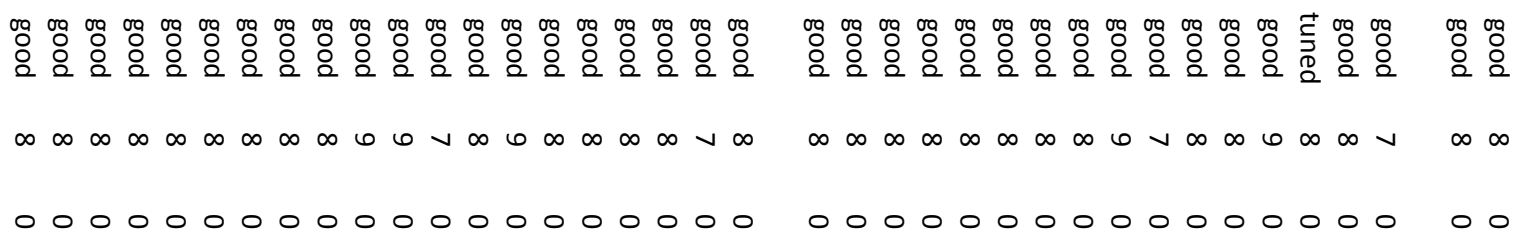

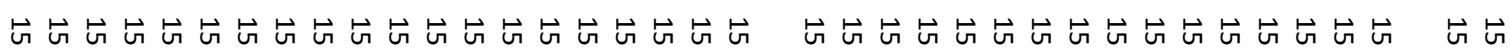

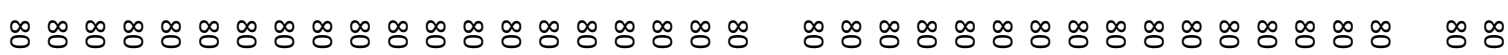

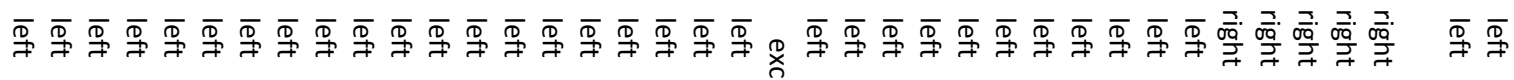

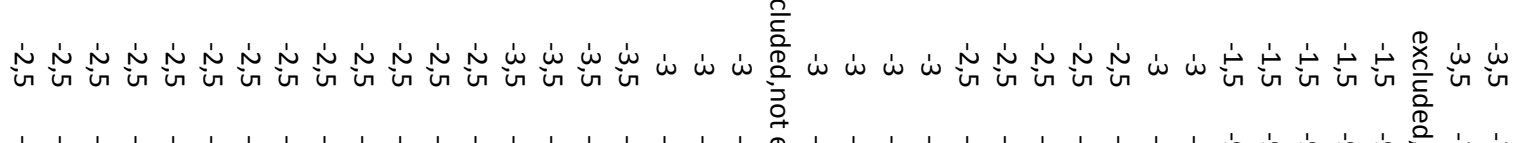

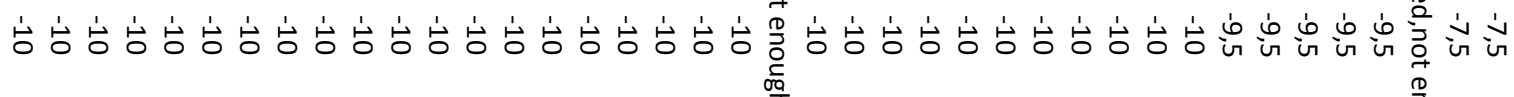

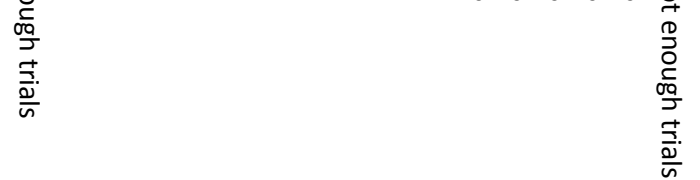

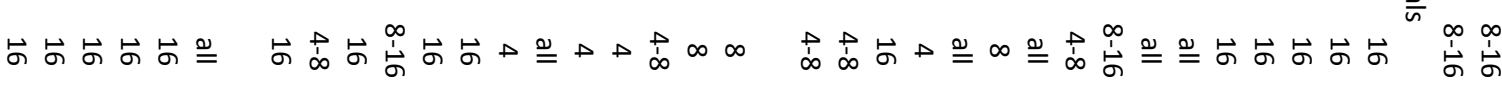

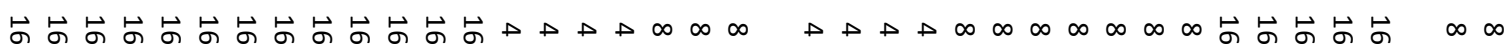

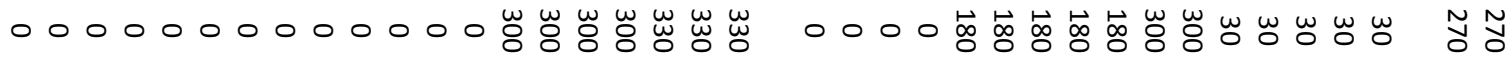

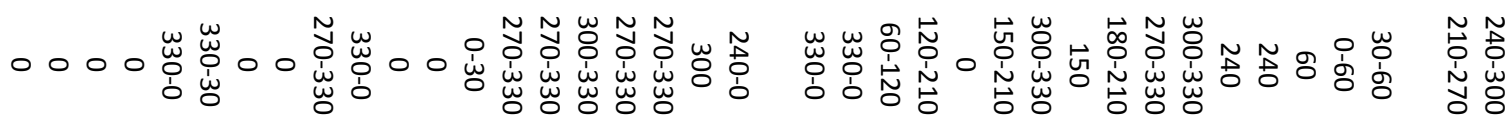

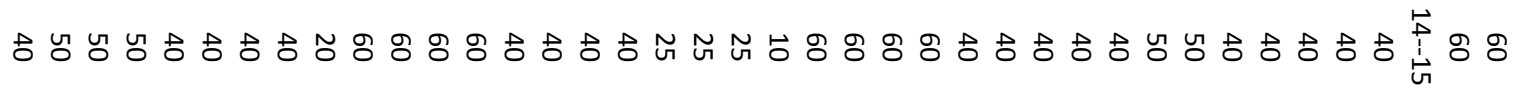




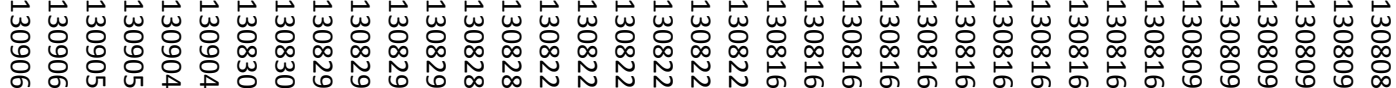

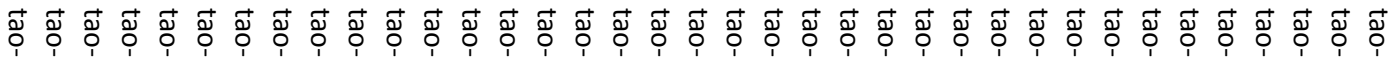

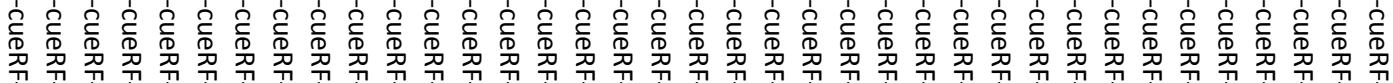

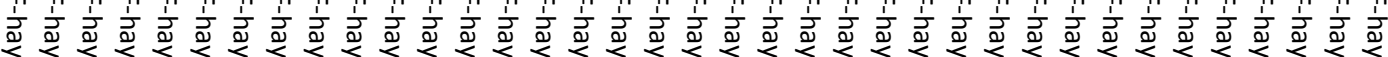

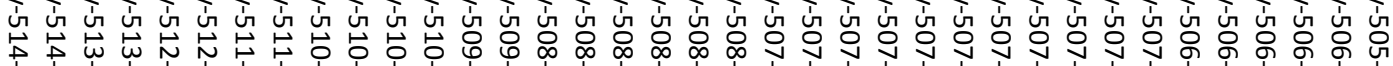

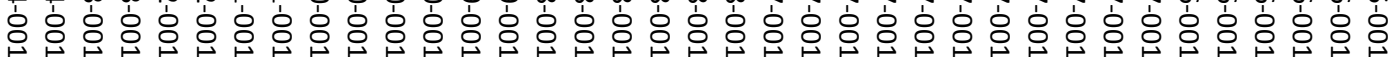

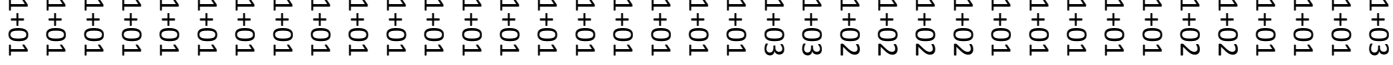

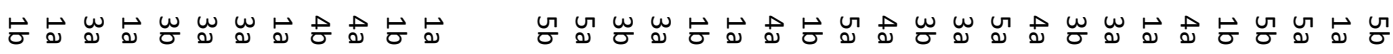

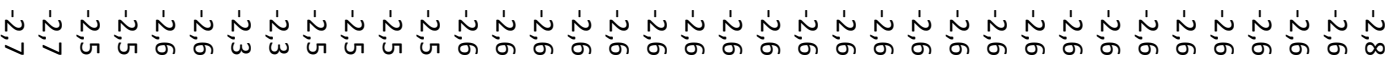

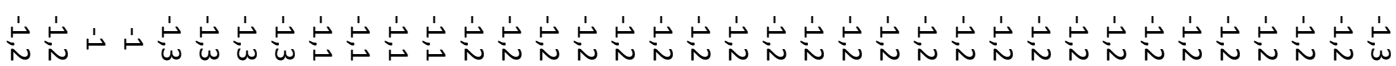

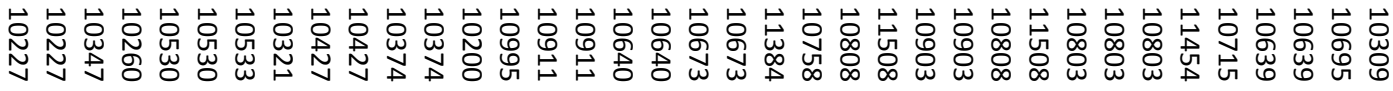

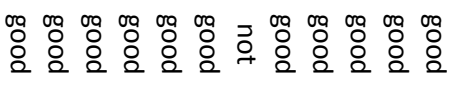

$\infty \quad \infty \quad \infty \quad \infty \quad \infty \quad \infty \quad \infty \quad \infty \quad \infty \quad \bullet \vee \infty$

000000000000

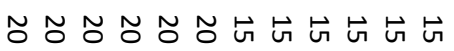

ஜ

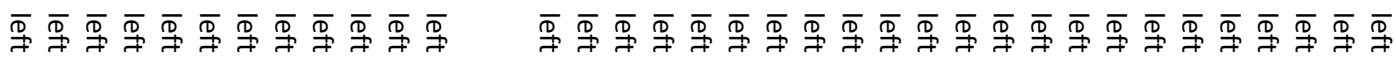

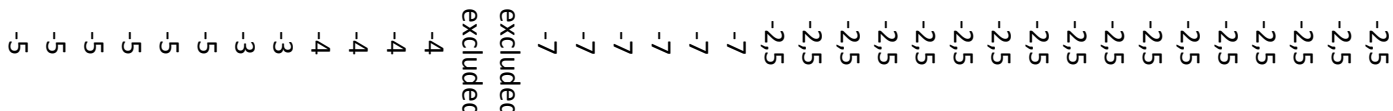

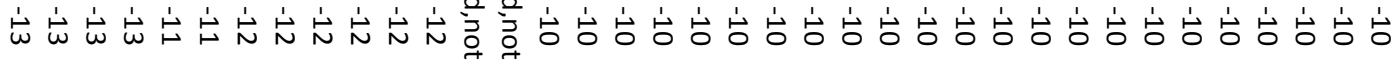

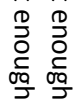

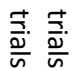

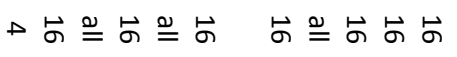
它 它

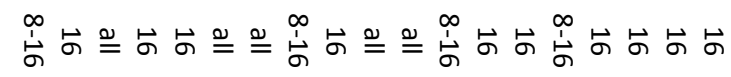

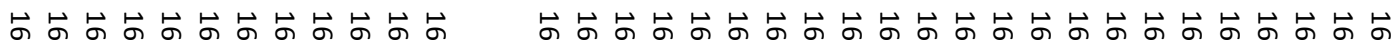

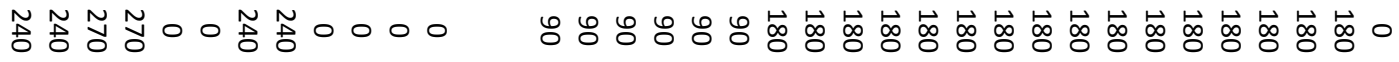

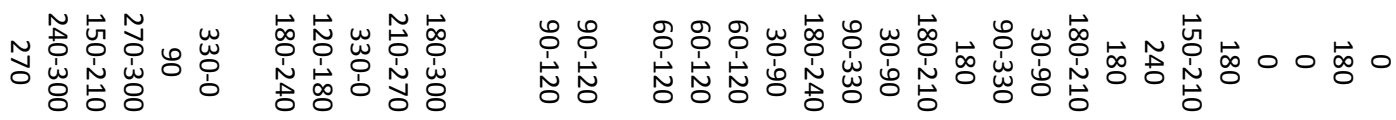

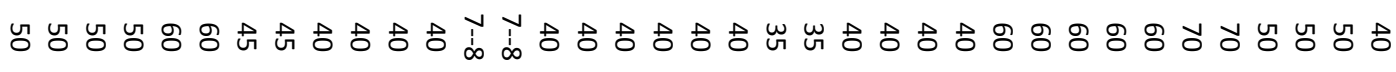

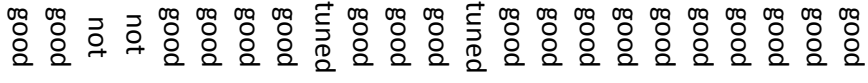

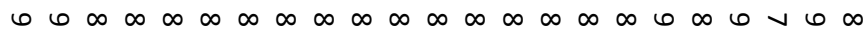

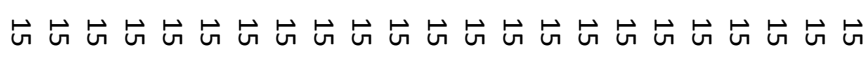

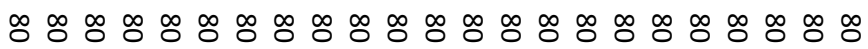
(1) 
Additional supplementary materials 3: Example epars \& mpars

Note: The epars define the time sequence of the experiment, the location of the stimulus, i.e. when and where to show the stimulus on the screen. The epars also on-line control the data collection and some events related to subject performance (such as eye position, time window of reaction etc.). The mpars controls the properties of the stimuli, such as the motion direction, speed, dot density, color of the RDP.

\section{Example epars:}

\section{Experiment 1 cueRF.epar}

projectLeader tao expCode cueRF

displayWindowOnSecondMonitor 00200200 trialProtocol 115

000010

$\begin{array}{llllll}55 & 0 & -40 & -360 & 1 & 1\end{array}$

$\begin{array}{llllll}10 & 20 & -40 & -360 & 1 & 1\end{array}$

1000000

$\begin{array}{llllll}55 & 0 & -40 & 360 & 1 & 1\end{array}$

$\begin{array}{llllll}10 & 20 & -40 & 360 & 1 & 1\end{array}$

10500011

$\begin{array}{llllll}55 & 59 & -40 & -360 & 1 & 1\end{array}$

$\begin{array}{lllllll}55 & 59 & -40 & 360 & 1 & 1\end{array}$

$\begin{array}{llllll}55 & 0 & -40 & -360 & 1 & 0\end{array}$

$\begin{array}{llllll}55 & 0 & -40 & 360 & 1 & 0\end{array}$

$\begin{array}{llllll}55 & 59 & -40 & -360 & 1 & 0\end{array}$

$\begin{array}{llllll}55 & 59 & -40 & 360 & 1 & 0\end{array}$

$\begin{array}{llllll}10 & 20 & -40 & -360 & 1 & 0\end{array}$

$\begin{array}{llllll}10 & 20 & -40 & 360 & 1 & 0\end{array}$

\# 2 stimuli in FRF \# 3 cue in FRF;

\# 4 1st FP; 5 patch opposite to FRF; 6 cue for 5; 7 2nd FP or saccade target;

\# 8 and 9 patches removed before saccade in FRF;

\# 10 and 11 patches in RF; 12 and 13 patches removed before saccade in $\mathrm{RF}$.

$\begin{array}{llll}\text { displayCenter } 0 & 1 & 0.65 & 0.5\end{array}$

displayCenter $1 \quad 1 \quad-6000$

filenames\&pathname 37 :traceAtt:traceMpars:

Luminance.mpar

fixStim1.mpar

fixStim2.mpar

Cue.mpar

TP.mpar

T45.mpar

T90.mpar
T135.mpar

T180.mpar

T225.mpar

T270.mpar

T315.mpar

DP.mpar

D45.mpar

D90.mpar

D135.mpar

D180.mpar

D225.mpar

D270.mpar

D315.mpar

AttTP.mpar

AttDP.mpar

DPshort.mpar

fixStimlong.mpar

DPOff.mpar

AttTPF.mpar

AttDPF.mpar

D1800ff.mpar

fixStim4.mpar

fixStim5.mpar

fixStim6.mpar

AttTP180.mpar

AttDP180.mpar

AttTPF180.mpar

AttDPF180.mpar

Att4TP.mpar

Att4DP.mpar

\# four patches, cue in FRF(class 1-4) or

RF(class 6-9)

\# class 1 and 2 cue in and cue out contineous, cue in FRF, class $3=1$, class $4=2$

\# class 5 and 6 cue in and cue out interrupted, cue in RF

\# class 7 and 8 pure saccade

classes 9

1000100000000000000

1293700370310036370040100

1293700370310037360004200

1293700370310036370040300

1293700370310037360004400

12000003252500252540500

12000003252500252504600

1200000300000000700

1200000300000000800

classFrequencyOption 0

\# default is zero, this equates the number of trials

waitForLever 10000

rewardDuration 90 
leverDownRewardDuration 0 preLeverBeepDelay 2000 anticipatedResponseDuration 150 responseTimeWindow 600 \#changed above from 500 hitDelay 1500

missDelay 1000

leverReleaseDuration 600 juicePin 1

fixpointIndexColor 255

backgroundIndexColor 200

fixPointSize 12

keyboardResponse FALSE

earlyResponsePermitted FALSE

fixPointVisibleBetweenTrials false

fixationRequired FALSE

collectEyePositions TRUE

fixAreaRadiusOfClass 080

preLeverFixRelaxation 2

smoothEyePositions 4

spikeSource 1

sampleSource 1

collectSpikes TRUE

numberOfTrials 9000

dotPosToBuffer FALSE

spikeRecSystem 3

eyePosDecay -20

provideWords TRUE

openDataFile

savePupilDiameter TRUE

\section{Experiment 2}

\section{cueFRF.epar}

projectLeader tao

expCode cueFRF

displayWindowOnSecondMonitor 00200200

trialProtocol 115

0000010

$\begin{array}{llllll}55 & 0 & -40 & -360 & 1 & 1\end{array}$

$\begin{array}{llllll}10 & 20 & -40 & -360 & 1 & 1\end{array}$

1000000

$\begin{array}{llllll}55 & 0 & -40 & 360 & 1 & 1\end{array}$

$\begin{array}{llllll}10 & 20 & -40 & 360 & 1 & 1\end{array}$

10500011

$\begin{array}{llllll}55 & 59 & -40 & -360 & 1 & 1\end{array}$

$\begin{array}{llllll}55 & 59 & -40 & 360 & 1 & 1\end{array}$

$\begin{array}{llllll}55 & 0 & -40 & -360 & 1 & 0\end{array}$

$\begin{array}{lllllll}55 & 0 & -40 & 360 & 1 & 0\end{array}$

$\begin{array}{llllll}55 & 59 & -40 & -360 & 1 & 0\end{array}$

$\begin{array}{llllll}55 & 59 & -40 & 360 & 1 & 0\end{array}$

$\begin{array}{llllll}10 & 20 & -40 & -360 & 1 & 0\end{array}$

$\begin{array}{llllll}10 & 20 & -40 & 360 & 1 & 0\end{array}$

\# 2 stimuli in FRF \# 3 cue in FRF;
\# 4 1st FP; 5 patch opposite to FRF; 6 cue for 5; 7 2nd FP or saccade target;

\# 8 and 9 patches removed before saccade in FRF;

\# 10 and 11 patches in RF; 12 and 13 patches removed before saccade in $\mathrm{RF}$.

$\begin{array}{lccc}\text { displayCenter } 0 & 1 & 0.34375 & 0.5\end{array}$

displayCenter $1 \quad 1600 \quad 0$

filenames\&pathname 37 :traceAtt:traceMpars:

Luminance.mpar

fixStim1.mpar

fixStim2.mpar

Cue.mpar

TP.mpar

T45.mpar

T90.mpar

T135.mpar

T180.mpar

T225.mpar

T270.mpar

T315.mpar

DP.mpar

D45.mpar

D90.mpar

D135.mpar

D180.mpar

D225.mpar

D270.mpar

D315.mpar

AttTP.mpar

AttDP.mpar

DPshort.mpar

fixStimlong.mpar

DPOff.mpar

AttTPF.mpar

AttDPF.mpar

D1800ff.mpar

fixStim4.mpar

fixStim5.mpar

fixStim6.mpar

AttTP180.mpar

AttDP180.mpar

AttTPF180.mpar

AttDPF180.mpar

Att4TP.mpar

Att4DP.mpar

\# class 1 and 2 \& class 3 and 4 cue in and cue out contineous, cue in FRF

\# class 5 and 6 cue in and cue outinterrupted, cue in $\mathrm{RF}$

\# class 7 and 8 pure saccade

\# class 9 and 10 stimuli in FRF, no stimuli in $\mathrm{RF}$, sever as control for response to stimuli in FRF

classes 11 
1000100000000000000

1293640370310037370000100

1293700364310037370000200

1293640370310037370000300

1293700364310037370000400

12040003252500252500500

12000043252500252500600

1200000300000000700

1200000300000000800

1303640370000000000900

13037003640000000001000

classFrequencyOption 0

\# default is zero, this equates the number of

\section{trials}

waitForLever 10000

rewardDuration 90

leverDownRewardDuration 0

preLeverBeepDelay 2000

anticipatedResponseDuration 150

responseTimeWindow 600

\#changed above from 500

hitDelay 1500

missDelay 1000

leverReleaseDuration 600

juicePin 1

fixpointIndexColor 255

backgroundIndexColor 200

fixPointSize 12

keyboardResponse FALSE

earlyResponsePermitted FALSE

fixPointVisibleBetweenTrials false

fixationRequired FALSE

collectEyePositions TRUE

fixAreaRadiusOfClass 080

preLeverFixRelaxation 2

smoothEyePositions 4

spikeSource 1

sampleSource 1

collectSpikes TRUE

numberOfTrials 9000

dotPosToBuffer FALSE

spikeRecSystem 3

eyePosDecay -20

provideWords TRUE

openDataFile

savePupilDiameter TRUE

\section{Example mpars:}

\section{fixStim1.mpar}

numberOfSurfaces 1400

pixelradius 20

numberOfFrames 105

\#qtRGBForeColor

655365530
qtIndexForeColor 255

$\begin{array}{llllll}\text { qtSquare } & -6 & -6 & 6 & 6\end{array}$

\section{fixStim2.mpar}

numberOfSurfaces 1400

pixelradius 10

numberOfFrames 105

\#qtRGBForeColor 655365530

qtIndexForeColor 255

$\begin{array}{lllll}\text { qtSquare } & -6 & -6 & 6 & 6\end{array}$

qtSuspendFix 105125

qtFixFactor $\quad 105-1 \quad 1.5$

qtFixDispCtr $\quad 105-1 \quad 1$

\section{fixStim4.mpar}

numberOfSurfaces 1400

pixelradius 10

numberOfFrames 105

\#qtRGBForeColor

655365530

qtIndexForeColor 255

$\begin{array}{lllll}\text { qtSquare } & -6 & -6 & 6 & 6\end{array}$

qtSuspendFix 105125

qtFixFactor $\quad 105-1 \quad 1.5$

qtFixDispCtr $\quad 105-1 \quad 1$

\section{fixStim6.mpar}

numberOfSurfaces 1400

pixelradius 10

numberOfFrames 300

\#qtRGBForeColor

qtIndexForeColor 255

$\begin{array}{llllll}\text { qtSquare } & -6 & -6 & 6 & 6\end{array}$

qtSuspendFix 105125

qtFixFactor $\quad 105-1 \quad 1.5$

qtFixDispCtr $\quad 105-1 \quad 1$

\section{AttTPF.mpar}

numberOfSurfaces 14010

dotSize 4

pixelradius 80

numberOfFrames 250

speed 16

shape circle

colorIndex 255

direction 210

changePhase -60 140

directionTable $2120 \quad 300$

changePhase 1010

direction 210

\section{Att4TP.mpar}

numberOfSurfaces 14010

dotSize 4

pixelradius 80

numberOfFrames 250 
speed 16

shape circle

colorIndex 255

direction 90

changePhase -20 150

directionTable 20180

changePhase 1010

direction 90

\section{Att4DP.mpar}

numberOfSurfaces 14010

dotSize 4

pixelradius 80

numberOfFrames 250

speed 16

shape circle

colorIndex 255

direction 90

changePhase 20150

directionTable 20180

changePhase 1010

direction 90 
Chapter 3

Visual attention is available at a task-relevant

location rapidly after a saccade

(elife, in press) 


\section{Visual attention is available at a task-relevant location rapidly after a saccade}

Tao $\mathrm{Yao}^{1 *}$, Madhura Ketkar ${ }^{1,2}$, Stefan Treue ${ }^{1,3,4}$, B. Suresh Krishna $^{1^{*}}$

Affiliations:

1 Cognitive Neuroscience Laboratory, German Primate Center, 37077 Goettingen, Germany

2 European Neuroscience Institute, 37077 Goettingen, Germany

3 Bernstein Center for Computational Neuroscience, 37077 Goettingen, Germany

4 Faculty of Biology and Psychology, Goettingen University, 37073

Goettingen, Germany

*taoyao@dpz.eu (TY), skrishna@dpz.eu (BSK)

Corresponding Authors:

Tao Yao,

B. Suresh Krishna,

Cognitive Neuroscience Laboratory,

German Primate Center,

Cognitive Neuroscience Laboratory,

37077 Goettingen,

German Primate Center,

Germany 37077 Goettingen,

49-551-3851354

Germany

taoyao@dpz.eu

49-551-3851354

skrishna@dpz.eu 


\section{Abstract}

2

3 Maintaining attention at a task-relevant spatial location while making eye-

4 movements necessitates a rapid, saccade-synchronized shift of attentional

5 modulation from the neuronal population representing the task-relevant

6 location before the saccade to the one representing it after the saccade.

7 Currently, the precise time at which spatial attention becomes fully allocated

8 to the task-relevant location after the saccade remains unclear. Using a fine-

9 grained temporal analysis of human peri-saccadic detection performance in

10 an attention task, we show that spatial attention is fully available at the task-

11 relevant location within 30 milliseconds after the saccade. Subjects tracked

12 the attentional target veridically throughout our task: i.e. they almost never

13 responded to non-target stimuli. Spatial attention and saccadic processing

14 therefore co-ordinate well to ensure that relevant locations are attentionally

15 enhanced soon after the beginning of each eye fixation. 


\section{Introduction}

3 The processing of vision and visuospatial attention mostly proceeds via

4 retinotopic representations in the brain $[1,2]$. Since each saccadic eye-

5 movement leads to a change in the retinotopic representation of the visual

6 scene, maintaining attention at a task-relevant spatial location across a

7 saccade necessitates a rapid, saccade-synchronized shift of attentional

8 modulation from the neuronal population representing the task-relevant

9 location before the saccade to the one representing it after the saccade [3,

10 4]. Currently, perceptual measurements in humans suggest a neuronal

11 attention shift that starts before the saccade and continues after the saccade

12 ends [5-10]. However, because these previous measurements used coarse

13 temporal sampling and/or long-duration attentional probes, the precise time

14 at which spatial attention becomes fully allocated to the task-relevant location

15 after the saccade remains unclear. Here, using a fine-grained temporal

16 analysis of human peri-saccadic detection performance in an attention task,

17 we show that spatial attention is fully available at the task-relevant location

18 within 30 milliseconds after the saccade. This rapid post-saccadic recovery of

19 performance in our attention task indicates that retinotopic attentional shifts

20 occur within the time required to recover from saccadic suppression of vision.

21 Subjects almost never responded to the distractor change, indicating that

22 they tracked the attentional target veridically throughout the task. Spatial

23 attention and saccadic processing therefore co-ordinate well to ensure that

24 relevant locations are attentionally enhanced soon after the beginning of

25 each eye fixation. 


\section{Results and Discussion}

2

3 We measured human peri-saccadic attentional allocation by combining an

4 endogenous spatial attention task with a visually-guided saccade. Human

5 subjects had to make a saccade to follow a fixation point when it jumped to a

6 new location, and concurrently, pay attention throughout the trial to a target

7 moving random-dot pattern (RDP) presented eccentrically among three or

8 five physically similar distractor RDPs (Figure 1, and Experimental

9 Procedures). We measured the subjects' attentional allocation by their ability

10 to detect a brief $(23.5 \mathrm{~ms})$ change in target motion, while ignoring similar

11 changes in the distractors. The target and distractor changes occurred at

12 different times around the saccade, allowing us to report for the first time,

13 peri-saccadic performance in an attention task with fine-grained temporal

14 precision. The intervening saccade poses a challenge for the attentional

15 system, because due to the retinotopic shift of the target location across the

16 saccade, the attentional system needs to shift its modulatory influence from

17 the neuronal population representing the target before the saccade to the

18 neuronal population representing the target after the saccade. By using a

19 fixed timing and location for the fixation point jump, we could isolate the

20 dynamics of this attentional remapping process and minimize its interaction

21 with the dynamics of attentional allocation to other exogenous visual events.

22 We therefore made the saccade spatially and temporally predictable by

23 having the fixation point jump at the same time and to the same location on

24 each trial so that the subject could best focus on the target pattern. 
1 In Experiment 1, we looked at the peri-saccadic performance of 8 subjects

2 (pooled data in Figure 2A, individual subject-data in Figure 2 - figure

3 supplement 1). At times well before and well after the saccade, subjects

4 almost always detected the target change and their performance was near

$5100 \%$. Performance began to drop around the time the fixation point jumped

6 (dashed vertical line in Figure 2A), as expected from the previously reported

7 diversion of pre-saccadic attentional resources towards the saccade task [11-

8 13] and the post-saccadic retinotopic location [6]. The performance then

9 dropped steeply right before the saccade, as expected from the drop in visual

10 sensitivity due to saccadic suppression [14-16]. Importantly, our data show

11 (for the first time in an attention task, to our knowledge) that performance

12 recovered back to baseline within $30 \mathrm{~ms}$ of saccade offset (Figure $2 \mathrm{~A}$ ). The

13 rapid post-saccadic recovery of performance indicates that attention is

14 allocated to the task-relevant location within $30 \mathrm{~ms}$ after the saccade ends.

15 The rapid time-course of recovery resembles that previously shown for

16 saccadic suppression of visual performance in tasks where visual sensitivity

17 was probed around a saccade using a briefly flashed change, but without any

18 requirement to maintain attention on a target across a saccade [14-16]. This

19 suggests that while resumption of visual function after a saccade is

20 constrained by the recovery from saccadic suppression [17], the peri-

21 saccadic attentional shift necessitated by retinotopic visual processing does

22 not impose an additional temporal cost on this recovery. The rapid post-

23 saccadic attentional availability at the target location that we infer from our

24 data is consistent with the only physiological data on this issue: in a mental

25 curve-tracing task similar to ours with a fixed attentional target, attentional

26 effects in monkey $\mathrm{V} 1$ emerge approximately $80 \mathrm{~ms}$ after the end of the 
1 saccade [18]. Given an onset latency of approximately 30 to $50 \mathrm{~ms}$ in

2 monkey V1, MT and LIP [18-20], a change occurring $30 \mathrm{~ms}$ after saccade

3 offset would reach the visual cortex at approximately the time when its

4 neurons representing the target after the saccade are attentionally enhanced.

6 It is possible that though we report a rapid recovery in Experiment 1, the true

7 recovery was actually slower, but was masked by the fact that performance

8 had already reached its maximum value of $100 \%$ within 30 ms of saccade

9 offset. We therefore performed a similar experiment (Experiment 2) with two

10 task difficulties, where peak performance on the harder task was clearly

11 below 100\% (Figure 2B). Once again, performance recovered to baseline

12 levels within $30 \mathrm{~ms}$ of saccade offset in both the easier and the harder task,

13 indicating that our estimate of a rapid recovery time for performance was

14 genuine and not an artifact due to a ceiling effect. The recovery time-course

15 after the saccade also did not seem to depend on saccade latency (Figure

16 3). Very similar performance was observed when we grouped trials based on

17 saccade latency into three groups: putative predictive saccades (latencies

18 from 0 to $75 \mathrm{~ms}$ ), express saccades (latencies from 75 to $125 \mathrm{~ms}$ ) and

19 regular-latency saccades (latencies from 125 to $250 \mathrm{~ms}$ ). This indicates that

20 though various differences between these different kinds of saccades have

21 been noted and these different kinds of saccades have been speculated to

22 arise via different neural pathways [21-27], peri-saccadic attentional shifts

23 seem to proceed with a similar time-course in each case.

25 If the peri-saccadic attentional shift is not temporally well-synchronized with

26 the saccade, attention will be peri-saccadically allocated to irrelevant spatial 
1 locations. In fact, prior findings measuring discrimination performance for

2 attentional probes at different locations suggest that by about $75 \mathrm{~ms}$ before

3 the saccade, attentional enhancement could be seen at the "post-saccadic"

4 retinotopic location (which would be the wrong pre-saccadic spatial location)

5 [5]. Other studies report that after the saccade, attention stays at the pre-

6 saccadic retinotopic location (which would be the wrong post-saccadic spatial

7 location) for up to $100 \mathrm{~ms}$ after the saccade $[7,28]$. The predictive

8 emergence of attention is consistent with single-neuron data from monkeys

9 showing predictive responses in different attentional control areas of the

10 brain [2, 29], while imaging data from humans have been presented as

11 evidence for persistent retinotopic neural activity [9]. Results from a more

12 recent detailed study indicate that peri-saccadic attentional spread and

13 dynamics may show complex patterns: patterns consistent with predictive

14 shifts, transient retinotopic persistence as well as rapid post-saccadic

15 availability of attention at the task-relevant location were seen [10]. In our

16 data, we found no effect of predictive or delayed shifts on the rate of

17 responding to distractor changes (false-positives). In both Experiments 1 and

182 , overall, subjects responded to a distractor change on only 2.2 and $2 \%$ of

19 trials respectively. Distractor changes occurred either at the distractor

20 vertically below the target (a control) or at the distractor to the right of the

21 target (that tested post-saccadic retinotopic persistence of the pre-saccadic

22 attentional focus). In the time interval immediately after the saccade (0-150

$23 \mathrm{~ms}$ ), the data from both experiments showed no statistically significant

24 increase in the rate of false-positives due to retinotopic persistence

25 (compared to the control location; all p-values >0.16, Boschloo's test;

26 Supplementary file 1A). An additional experiment (Experiment 3; Figure 2 - 
1 figure supplement 3 and Supplementary file 1B) where we changed the task

2 design to test both pre-saccadic predictive shifts and post-saccadic

3 retinotopic persistence (while making distractor changes more numerous and

4 salient) also led to a false-positive rate of less than $1.4 \%$ and no evidence

5 for an effect of either predictive shifts or retinotopic persistence on the false-

6 positive rate. Subjects thus tracked the attentional target veridically

7 throughout our task, and the peri-saccadic spread of attention to irrelevant

8 spatial locations reported in previous studies does not seem to have any

9 manifest effects in our task. One important difference between our task and

10 previous tasks was that we included only one attended location within each

11 trial, and stimuli at all other locations were distractors that the subject had to

12 ignore. In contrast, the previous tasks required subjects to report a probe

13 stimulus that could appear at any of the stimulus locations. There were no

14 distractor stimuli, and attention was instead manipulated by using a dual-task

$15[5,7,28]$ or using an exogenous cue [10]. The fact that all stimulus locations

16 on each trial were potential targets in the previous studies may have led the

17 subjects to adopt a different attentional-set compared to the subjects in our

18 study. Alternatively, the previous results may have reflected only an

19 attentional effect on probe visibility, while the results in our task may

20 additionally reflect the effect of attention on distractor filtering. In current

21 theoretical accounts of attention [30, 31], the effects of attention on distractor

22 filtering and probe visibility correspond to the distinct effects of attention on

23 selection/weighting and sensory signal enhancement respectively. In this

24 scenario, distractor filtering due to the attentional selection/weighting of

25 sensory signals across the visual field is well-synchronized to the saccade

26 and therefore does not spread to irrelevant spatial locations. In contrast, 
1 attentional signal enhancement, but not distractor filtering, is influenced by

2 the predictive shifts and post-saccadic retinotopic persistence of attentional

3 modulation in the brain. As a result, in the previous tasks without a distractor

4 filtering component, the perceptual visibility of probes at irrelevant locations

5 was improved. In our task, any enhanced sensory signal from distractor

6 locations would continue to be down-weighted and filtered out and the

7 subjects would not respond to them. We emphasize that this is only one

8 plausible explanation, and theoretical models of attention are sufficiently

9 complex and flexible to admit alternative explanations. Even more generally,

10 the observed differences could be a result of task-dependent (or even

11 entirely different) attentional mechanisms operating in the different tasks.

12 Extensive measurements and model-testing will be necessary to

13 disambiguate the different possibilities.

15 Our data represent an important advance in the ongoing discussion about 16 the shifts of spatial attention around the time of a saccade [1, 3, 32-34]. We 17 provide the first temporally fine-grained measurements of detection 18 performance in an attention task in the critical immediate post-saccadic 19 period (0 to $100 \mathrm{~ms}$ following saccade offset). Our data show that 20 performance fully recovers soon after the end of the saccade, indicating that 21 the correct stimulus is attended to during this immediate post-sacadic period 22 when visual sensitivity is known to be highest [35]. The rapid time-course of 23 recovery resembles the time-course previously shown for the recovery of 24 visual function from saccadic suppression, suggesting that the retinotopic 25 attentional shift does not impose an additional temporal cost on the 26 resumption of visual function after a saccade. Further, our data indicate that 
1 under our task conditions, subjects very rarely confuse a distractor stimulus

2 for the target. Spatial attention and saccadic execution thus appear to co-

3 ordinate well to ensure that relevant objects are attentionally enhanced soon

4 after the beginning of each eye fixation. These findings are likely to lead to a

5 much better understanding of the impact of peri-saccadic changes in neural

6 activity on visual processing. 


\section{Materials and Methods}

3 We measured peri-saccadic attentional allocation by combining a spatial

4 attention task with a visually-guided saccade. We asked human observers to

5 make a saccade to a visual target, and within the same trial, also report a

6 speed and direction change in a target moving random-dot pattern (RDP),

7 while ignoring a similar change in one of the simultaneously-presented

8 distractor RDPs. The target and distractor changes occurred at different

9 times around the saccade, allowing us to measure peri-saccadic attentional

10 performance with fine-grained temporal precision.

Human Subjects

1410 subjects (4 males, 6 females, ages 21-30 years) participated in the study, 15 including two of the authors (MK and TY). 8 of the subjects (excluding the 16 two authors) were naïve to the purpose of the experiment. 8,5 and 4 17 subjects participated in Experiments 1,2 and 3 respectively; of these, 3 18 subjects (including the author MK) participated in all 3 experiments. All 19 subjects were right-handed and reported normal or corrected to normal 20 vision. All naive participants received monetary compensation for each 21 session. Each subject started the experiment with a training session to 22 become familiar with the tasks. The experiments were performed in several 23 blocks over one or two days. Subjects were given verbal and written 24 instructions about the task. The study was performed in accordance with 25 institutional guidelines for experiments with humans, adhered to the 26 principles of the Declaration of Helsinki and was approved by the Ethics 
1 Committee of the Georg-Elias-Müller-Institute of Psychology, University of

2 Göttingen. Each subject gave informed written consent prior to participating

3 in the study.

4

5 Apparatus

6

7 Subjects were seated in a dimly lit room at a viewing distance of $57 \mathrm{~cm}$ from

8 the screen with their head resting on a chin and forehead-rest. The only light

9 source in the room was the light from the display monitor. A computer

10 keyboard was used for recording subject responses. All aspects of the

11 experiment were controlled by custom software running on an Apple

12 Macintosh computer. The eye-position was monitored by an infra-red video-

13 based eye-tracker (iView X software running on a SMI Hi-Speed 1250

14 tracker, SMI GmbH, Germany) at $1000 \mathrm{~Hz}$. The stimuli were displayed on a

151600 by 1200 pixels ( 40 by 30 degrees) CRT monitor with a fresh rate of 85

$16 \mathrm{~Hz}$. The display background was always grey $\left(40 \mathrm{~cd} / \mathrm{m}^{2}\right)$, and all the visual

17 stimuli were black $\left(0.7 \mathrm{~cd} / \mathrm{m}^{2}\right)$.

18

Task design

21 We describe Experiment 1 first: Experiments 2 (Figure 2B) and 3 (Figure 2 -

22 figure supplement 3) are variants of Experiment 1.

23

24 Experiment 1: Each trial was started by the subject pressing the space-bar. A 25 fixation point appeared on the screen and subjects maintained their gaze 26 within 2 degrees of this point. The subjects concurrently performed a spatial 
1 attention task and a saccade task on each trial: they were instructed to pay

2 attention to the target RDP and make a saccade if the fixation point jumped

3 to a new location. For the spatial attention task, after $647 \mathrm{~ms}$ of fixation, four

4 circular moving random-dot patterns (RDPs: each presented within a circular

5 aperture of 2 degree radius, with dots moving upwards with a speed of 8

6 degrees of visual angle per second; dot density=10 dots per $\operatorname{deg}^{2}$ ), were

7 displayed on the screen. Individual RDP dot size was 0.15 degrees $\times 0.15$

8 degrees. The subjects were instructed that the RDP at a pre-specified

9 location (3.5 degrees to the right and 4 degrees above the fixation point:

10 Figure 1) was the target: they had to pay attention to that stimulus throughout

11 the trial in order to respond by pressing the downward-arrow key within 600

12 ms when they detected a brief 2-frame (23.5 ms) speed and direction-change

13 in the target RDP. For these two frames, the RDP dots moved faster at 16

14 degree per second and horizontally either to the left or the right, and then

15 resumed motion with the original speed and direction. Any changes in the

16 distractor RDPs were to be ignored. The median reaction-time was $324 \mathrm{~ms}$.

17 The second RDP was placed 7 degrees to the right of the target RDP so that

18 post-saccadically, it had the same retinotopic location as the target RDP did

19 pre-saccadically. The other two RDPs were placed at corresponding

20 locations in the lower hemifield. A target RDP change occurred on about $90 \%$

21 of the trials and between 118 to 1882 ms after RDP onset; the remaining

22 trials were catch trials and no change occurred. A distractor change occurred

23 before the target change on about $39 \%$ of trials, over a similar range of

24 times but at least $400 \mathrm{~ms}$ before the target change: the subject had to ignore

25 these changes. Only one target change and possibly also one distractor

26 change occurred on each trial. The distractor change could occur either at 
1 the RDP to the right of the target (with a post-saccadic retinotopic location

2 identical to the target's pre-saccadic retinotopic location) or the RDP below

3 the target. For the saccade task, the fixation point jumped to a new location 7

4 degrees horizontally to the right of the fixation point $1235 \mathrm{~ms}$ after the fixation

5 point appeared. The subjects had to make a single saccade within $553 \mathrm{~ms}$ of

6 the fixation point jump to fixate the saccade target location and then maintain

7 their gaze within 2 degrees of the saccade target for the remainder of the trial.

8 Most saccades occurred with a much shorter latency (Figure 3). The use of a

9 predictable time at which the fixation point jumped was advantageous

10 because subjects could focus their attention better on the target RDP without

11 worrying about the temporal uncertainty about when the fixation point would

12 jump. Trials were terminated when the subject pressed the downward arrow

13 key, broke fixation or failed to press a key within $600 \mathrm{~ms}$ of a change in the

14 target RDP. Subjects received no other feedback about trial outcome. The

15 use of a 2 degree fixation window during the two fixation periods was not

16 critical. We also obtained similar results when using a narrower fixation

17 window of 0.5 degrees: we ensured that the eye did not deviate by more than

180.5 degrees from the median horizontal and vertical eye-position during

19 fixation on each trial (Figure 2 - figure supplement 5). Using the median eye-

20 position compensates for across-trial drifts in calibration and is based on the

21 standard calibration assumption that normal-viewing subjects will foveate a

22 visual target when asked to fixate on it and therefore, their eye-position

23 variability will be centered on the fixated location.

25 Experiment 2: This was similar to Experiment 1, with the following key 26 differences. Two task difficulties were used and the change involved only a 
1 motion direction change, without a speed change. The two task difficulties

2 were created by using two magnitudes of direction change for each subject;

3 these magnitudes were chosen in a separate calibration session, where the

4 overall detection performance was estimated for nine direction-change

5 magnitudes between 20 and 90 degrees. The calibration session used a

6 fixation task similar to the task in Experiment 2 except that no saccade was

7 required. The direction-changes that led to approximately $70 \%$ and $90 \%$

8 detection performance were chosen for Experiment 2. Across subjects, the

9 direction-change varied between 35 and 60 degrees for the hard task and 10 between 50 and 90 degrees for the easier task. Also, to make more of the

11 target changes occur peri-saccadically, the timing of the task was slightly 12 modified so that the RDPs came on at $412 \mathrm{~ms}$ after fixation point onset 13 (Figure 1), and the target motion change occurred from $235 \mathrm{~ms}$ to $1647 \mathrm{~ms}$ 14 after RDP onset; approximately $27 \%$ of trials had a distractor change before 15 the target-change over a similar time-frame (118 ms to $1224 \mathrm{~ms}$, with the 16 same constraint of a $400 \mathrm{~ms}$ separation from the target-change as in 17 Experiment 1). About $7 \%$ of trials were catch trials.

19 Experiment 3: This was also similar to Experiment 1, except that we used 2 20 additional distractor RDPs, giving a total of 6 RDPs instead of 4 . One of the 21 additional RDPs was placed seven degrees to the left of the target RDP, 22 which is the location to which attention would be expected to predictively 23 switch just before the saccade. The other RDP was placed eight degrees 24 below this RDP, in line with the other RDPs in the lower hemifield. Further, to 25 make the distractor changes more salient and improve the chances of a 26 false-positive, the speed now increased during the motion change from 4 to 
132 degrees per second (instead of 8 to 16 degrees per second in Experiment

2 1); the direction-change remained at 90 degrees (vertically upward to

3 horizontal towards the left or the right). The range of target change times was

4 slightly delayed compared to Experiments 1 and 2 so that a distractor change

5 could occur more often before a target change and a false positive potentially

6 elicited: the target changes in Experiment 3 could occur from 412 to $2176 \mathrm{~ms}$

7 after RDP onset. The distractor change occurred from 470 to $941 \mathrm{~ms}$ after

8 RDP onset so that the distractor changes now occurred more often (about

$960 \%$ of target-changes were now preceded by distractor changes) and

10 mostly before the saccade. Distractor changes occurred either to the right of

11 the target (to measure post-saccadic retinotopic persistence) or to the left of

12 the target (to measure pre-saccadic predictive shifts). About $6 \%$ of trials

13 were catch trials.

15 Data analysis

17 Data processing was done using MATLAB (Mathworks Inc, Natwick, MA), 18 except for the exact test of binomial proportions performed using the Exact 19 package [36] in $R$ [37]. We detected saccades using a standard velocity20 threshold algorithm: onset (and offset) times were determined by when the 21 eye velocity exceeded (and then dropped below) an individualized threshold 22 (set to between 40 and 70 degrees per second, fixed for each subject). This 23 threshold value was set to lie clearly above the peak excursions of the 24 baseline noise in the eye-velocity traces, and the algorithm was validated by 25 visual inspection for each subject. By considering the saccade to have ended 26 when the velocity dropped below a threshold value well above the baseline 
1 noise (and when the eye was still moving), our threshold criterion provides a

2 conservative, i.e. early definition of saccadic end-point and therefore a longer

3 estimate of the recovery time for perceptual performance. Our threshold-

4 setting detected the primary saccade close to its end, but excluded post-

5 saccadic dynamic overshoots or glissades [38, 39]. Setting a lower threshold

6 and including these small eye-movements led to an even lower estimate of

7 the recovery time of spatial attention (around $20 \mathrm{~ms}$, instead of the $30 \mathrm{~ms}$ we

8 report). We only included trials where the subjects made a single saccade to

9 the saccade target, and this saccade was made between $50 \mathrm{~ms}$ before and

$10450 \mathrm{~ms}$ after the time when the fixation point jumped. While these limits are

11 arbitrary, they are not critical and our results remain robust for other

12 reasonable choices, consistent with the lack of an effect of saccade latency

13 on performance (Figure 3).

15 Trials with a fixation break were excluded from further analysis. Early 16 responses before the target-change were extremely rare: early responses 17 constituted only $1.2,1$ and $0.7 \%$ of trials in Experiments 1,2 and 3 18 respectively, even when counting all early responses that were potentially 19 responses to the distractor change in this number. Responses to the 20 distractor change (false-alarms; see Results and Discussion) were also 21 extremely rare; we considered all early responses within $800 \mathrm{~ms}$ of a 22 distractor change as a response to the distractor. We could therefore exclude 23 trials with early responses as well and simply define performance using the 24 hit-rate (the proportion of target-changes that were correctly detected). We 25 plotted the performance as a function of the time of target-change relative to 26 saccade offset: since the speed and direction-change lasted 2 frames (at a 
1 refresh rate of $85 \mathrm{~Hz}$ ), we used the timing of the second frame to define the

2 time of target-change since this was the conservative choice given our focus

3 on the rapid performance recovery after the saccade. For the pooled

4 analyses (Figures 2, 3 and Figure 2 - figure supplement $3 A$ ), we pooled the

5 trials from all subjects and then calculated the mean and $95 \%$ Wilson-score

6 confidence intervals [40] over successive non-overlapping time-bins of the X-

7 axis variable (10 ms in Figure 2A, $20 \mathrm{~ms}$ in Figure 2B, $50 \mathrm{~ms}$ in Figure 3 and

$810 \mathrm{~ms}$ in Figure 2 - figure supplement $3 \mathrm{~A}$ ). To estimate the time at which

9 performance recovered to its post-saccadic baseline, we first estimated the

10 baseline performance (proportion of correct trials) from 100 to $500 \mathrm{~ms}$

11 following saccade offset and then compared this value (using Boschloo's

12 exact test of binomial proportions and a one-sided $p$-value for the peri-

13 saccadic performance being lower than the baseline performance) to the

14 performance in successive non-overlapping $10 \mathrm{~ms}$ time-bins from 0 to 100

15 ms following saccade offset. The starting-point of the first non-significant bin

16 (i.e. $p>0.1$ ) was taken as the time of recovery. Using a one-sided $p$-value and

17 a cutoff of 0.1 are both conservative choices in our situation since they would

18 only increase the estimated time of recovery. Using a cutoff of $p>0.05$ for

19 non-significance reduced the estimated time of recovery in Experiment 1

20 (Figure $2 \mathrm{~A}$ ) to $20 \mathrm{~ms}$, but did not affect any of the other estimates. Similarly,

21 the use of Boschloo's test also increases the power to detect a significant

22 difference, and is therefore conservative for our purposes [41, 42]. The time

23 estimated using $10 \mathrm{~ms}$ bins was further confirmed with a similar procedure

24 using $5 \mathrm{~ms}$ bins. In all cases (Figures $2 \mathrm{~A}, 2 \mathrm{~B}$ and 3 ), the estimated value was

$2530 \mathrm{~ms}$, meaning that the performance in the time-bin from both 30 to $35 \mathrm{~ms}$

26 and 30 to $40 \mathrm{~ms}$ was not significantly different from baseline. For Experiment 
11 (Figure 2A), there were at least 48 trials in each $10 \mathrm{~ms}$ time-bin from 0 to

$240 \mathrm{~ms}$. For the other experiments, the values were: Experiment 2 (Figure 2B)

$3-42$ trials for the easy task, and 39 trials for the hard task and Experiment 3

4 (Figure 2 - figure supplement 3) - 31 trials. These trial numbers gave us 80

$5 \%$ power to detect a reduction to $90 \%$ (Experiment 1), $90 \%$ (Experiment 2,

6 easy), $81 \%$ (Experiment 2, hard) and $83 \%$ (Experiment 3) of the baseline

7 value, and the estimated recovery times agreed well with the values one

8 would estimate based on visual inspection of the curves. For the individual

9 subjects (Figure 2 - figure supplements 1-3), the time-courses appear very

10 similar to the pooled averages. However, formal statistical testing was

11 precluded by the small number of trials in each bin, since the estimates of

12 recovery time based on statistical significance would be shorter than the

13 estimate for the pooled averages (and therefore anti-conservative). We

14 therefore marked the estimated time at which the performance reached $80 \%$

15 of the baseline probability on the individual subject plots in Figure 2 - figure

16 supplements 1 to 3 . This value was calculated via simple linear interpolation

17 and by visual inspection, captures the time-course of recovery quite well. We

18 collected data from a planned number of 8 subjects in Experiment 1 . Since

19 the data from the 8 subjects in Experiment 1 showed very similar time-

20 courses, we collected data from a smaller number of 5 and 4 subjects

21 respectively in the additional experiments (2 and 3). 


\section{Author contributions}

2

3 TY and BSK designed the study; TY and MK conducted the experiments; TY,

$4 \quad$ MK and BSK analyzed the data; ST commented on the manuscript; TY and

$5 \quad$ BSK wrote the manuscript.

6

7 Acknowledgments

8

9 This work was supported by the Deutsche Forschungsgemeinschaft through

10 the Collaborative Research Center 889 "Cellular Mechanisms of Sensory

11 Processing" to ST (Project C04), and by the Germany-Israeli Foundation for

12 Scientific Research and Development Grant No. 1108-79.1/2010. 
1. Cavanagh P, Hunt AR, Afraz A, Rolfs M. Visual stability based on remapping of attention pointers. Trends Cogn Sci. 2010;14(4):147-53.

2. Wurtz RH. Neuronal mechanisms of visual stability. Vision Res. 2008;48(20):2070-89.

3. Marino AC, Mazer JA. Perisaccadic Updating of Visual Representations and Attentional States: Linking Behavior and Neurophysiology. Front Syst Neurosci. 2016;10:3.

4. Yao T, Treue S, Krishna BS. An Attention-Sensitive Memory Trace in Macaque MT Following Saccadic Eye Movements. PLoS Biol. 2016;14(2):e1002390.

5. Rolfs M, Jonikaitis D, Deubel H, Cavanagh P. Predictive remapping of attention across eye movements. Nat Neurosci. 2011;14(2):252-6.

6. Szinte M, Carrasco M, Cavanagh P, Rolfs M. Attentional trade-offs maintain the tracking of moving objects across saccades. J Neurophysiol. 2015;113(7):2220-31.

7. Golomb JD, Chun MM, Mazer JA. The native coordinate system of spatial attention is retinotopic. J Neurosci. 2008;28(42):10654-62.

8. Golomb JD, Marino AC, Chun MM, Mazer JA. Attention doesn't slide: spatiotopic updating after eye movements instantiates a new, discrete attentional locus. Atten Percept Psychophys. 2011;73(1):7-14.

9. Golomb JD, Nguyen-Phuc AY, Mazer JA, McCarthy G, Chun MM. Attentional facilitation throughout human visual cortex lingers in retinotopic coordinates after eye movements. J Neurosci. 2010;30(31):10493-506.

10. Jonikaitis D, Szinte M, Rolfs M, Cavanagh P. Allocation of attention across saccades. J Neurophysiol. 2013;109(5):1425-34.

11. Deubel H, Schneider WX. Saccade target selection and object recognition: evidence for a common attentional mechanism. Vision Res. 1996;36(12):182737.

12. Montagnini A, Castet E. Spatiotemporal dynamics of visual attention during saccade preparation: Independence and coupling between attention and movement planning. J Vis. 2007;7(14):8 1-16.

13. Hoffman JE, Subramaniam B. The role of visual attention in saccadic eye movements. Percept Psychophys. 1995;57(6):787-95.

14. Diamond MR, Ross J, Morrone MC. Extraretinal control of saccadic suppression. J Neurosci. 2000;20(9):3449-55.

15. Dorr M, Bex PJ. Peri-saccadic natural vision. J Neurosci. 2013;33(3):12117.

16. McConkie GW, Loschky LC. Perception onset time during fixations in free viewing. Behav Res Methods Instrum Comput. 2002;34(4):481-90.

17. Krekelberg B. Saccadic suppression. Curr Biol. 2010;20(5):R228-9.

18. Khayat PS, Spekreijse H, Roelfsema PR. Correlates of transsaccadic integration in the primary visual cortex of the monkey. Proc Natl Acad Sci U S A. 2004;101(34):12712-7.

19. Bair W, Cavanaugh JR, Smith MA, Movshon JA. The timing of response onset and offset in macaque visual neurons. J Neurosci. 2002;22(8):3189-205. 
20. Bisley JW, Krishna BS, Goldberg ME. A rapid and precise on-response in posterior parietal cortex. J Neurosci. 2004;24(8):1833-8.

21. Bronstein AM, Kennard C. Predictive eye saccades are different from visually triggered saccades. Vision Res. 1987;27(4):517-20.

22. Chen M, Liu Y, Wei L, Zhang M. Parietal cortical neuronal activity is selective for express saccades. J Neurosci. 2013;33(2):814-23.

23. Cotti J, Panouilleres M, Munoz DP, Vercher JL, Pelisson D, Guillaume A. Adaptation of reactive and voluntary saccades: different patterns of adaptation revealed in the antisaccade task. J Physiol. 2009;587(1):127-38.

24. Deubel, H. ed. (1995). Is Saccadic Adaptation Context-Specific ? In Eye Movement Research - Mechanisms, Processes, and Applications. Findlay, J.M., Walker, R., and Kentridge, R.W., ed. (Elsevier Science), Volume 6, 177-187.

25. Gaymard B, Ploner CJ, Rivaud S, Vermersch AI, Pierrot-Deseilligny C. Cortical control of saccades. Exp Brain Res. 1998;123(1-2):159-63.

26. Pierrot-Deseilligny C, Ploner CJ, Muri RM, Gaymard B, Rivaud-Pechoux S. Effects of cortical lesions on saccadic: eye movements in humans. Ann N Y Acad Sci. 2002;956:216-29.

27. Shelhamer M, Joiner WM. Saccades exhibit abrupt transition between reactive and predictive; predictive saccade sequences have long-term correlations. J Neurophysiol. 2003;90(4):2763-9.

28. Golomb JD, Pulido VZ, Albrecht AR, Chun MM, Mazer JA. Robustness of the retinotopic attentional trace after eye movements. J Vis. 2010;10(3):19 1-2.

29. Duhamel JR, Colby CL, Goldberg ME. The updating of the representation of visual space in parietal cortex by intended eye movements. Science. 1992;255(5040):90-2.

30. Eckstein MP, Peterson MF, Pham BT, Droll JA. Statistical decision theory to relate neurons to behavior in the study of covert visual attention. Vision Res. 2009;49(10):1097-128.

31. Lu ZL, Dosher BA. External noise distinguishes attention mechanisms. Vision Res. 1998;38(9):1183-98.

32. Rolfs M, Szinte M. Remapping Attention Pointers: Linking Physiology and Behavior. Trends Cogn Sci. 2016.

33. Mayo JP, Sommer MA. Shifting attention to neurons. Trends Cogn Sci. 2010;14(9):389; author reply 90-1.

34. Melcher D. The missing link for attention pointers: comment on Cavanagh et al. Trends Cogn Sci. 2010;14(11):473; author reply 4-5.

35. Ibbotson M, Krekelberg B. Visual perception and saccadic eye movements. Curr Opin Neurobiol. 2011;21(4):553-8.

36. Calhoun P. Exact: Unconditional Exact Test. R package version 1.6. https://CRAN.R-project.org/package=Exact. 2015.

37. Team RC. R: A language and environment for statistical computing. R Foundation for Statistical Computing, Vienna, Austria. URL: https://http://www.R-project.org/. 2016.

38. Bahill AT, Clark MR, Stark L. Dynamic overshoot in saccadic eye movements is caused by neurological control signed reversals. Exp Neurol. 1975;48(1):107-22.

39. Nystrom M, Hooge I, Holmqvist K. Post-saccadic oscillations in eye movement data recorded with pupil-based eye trackers reflect motion of the pupil inside the iris. Vision Res. 2013;92:59-66. 
1 40. Brown LD, DasGupta A, Cai TT. Interval Estimation for a Binomial 2 Proportion. Statistical Science. 2001;16(2):101--33.

3 41. Berger RLRL. Power comparison of exact unconditional tests for 4 comparing two binomial proportions. Institute of Statistics Mimeo Series. 5 1994(2266).

6 42. Mehrotra DV, Chan ISF, Berger RL. A cautionary note on exact 7 unconditional inference for a difference between two independent binomial 8 proportions. Biometrics. 2003;59(2):441--50. 
3 Figure 1. Task-design and timing. Human subjects performed a task that

4 involved attending to a target (marked with a white $T$ and always at the same

5 location) presented as one among four (A) or six (B) moving RDPs while also

6 making a visually guided saccade if the fixation point jumped seven degrees

7 to the right (1235 ms after trial onset). The subjects were instructed to

8 respond with a key-press when the target RDP briefly changed speed and

9 direction, but to ignore similar changes in any of the remaining RDPs. Target

10 and distractor changes occurred at different times around the saccade,

11 enabling the measurement of peri-saccadic performance in this attention 12 task. Two different task-difficulties were used in Experiment 2, while six 13 RDPs were used in Experiment 3 instead of four. There were also minor 14 differences in timing between the three tasks.

16 Figure 2. Rapid post-saccadic recovery of performance. A) Detection17 performance (hit-rate) of motion-direction drops around the time of the 18 saccade and recovers within $30 \mathrm{~ms}$ after the saccade. The figure shows the 19 mean detection-performance (and $95 \%$ confidence bands) for all trials 20 pooled over 8 subjects calculated in non-overlapping $10 \mathrm{~ms}$ time-bins of the 21 abscissa (time of target-change relative to saccade offset). The inset shows 22 the same data, focusing on the time between -100 and $100 \mathrm{~ms}$. Data from 23 individual subjects show little inter-individual variability in the time-course of 24 recovery (figure supplement 1 ). The triangle indicates the earliest time (30 $25 \mathrm{~ms}$ ) at which performance is statistically indistinguishable from that over the 26100 to 500 ms time-period (using Boschloo's exact test; see Experimental 
1 Procedures). The dashed vertical line indicates the mean time of fixation-

2 point offset and the stippled vertical line indicates the mean saccade onset

3 time. See also figure supplements 1 and 3. B) Similar results were obtained

4 when two different task-difficulties were used (data pooled over 5 subjects).

5 The data from the higher-difficulty task (in red) show that the rapid recovery

6 is not an artifact of a ceiling effect on performance. Data plotted using $20 \mathrm{~ms}$

7 time-bins. Figure conventions as in Figure 2A. See also figure supplement 2

8 for data from individual subjects. Figure supplements 4 and 5 replot the same

9 data as in Figures $2 \mathrm{~A}$ and $2 \mathrm{~B}$ and in the same format, but figure supplement

104 uses the time of target-change relative to saccade onset and figure

11 supplement 5 only includes trials where a fixation window of 0.5 degrees was

12 used (see corresponding legends for details).

Figure 3. Rapid post-saccadic performance recovery is independent of saccade latency. The time-course of recovery was indistinguishable for

16 saccades in three different latency ranges in the same dataset used in Figure 17 2A (8 subjects, color coding in inset): 0-75 ms (predictive saccades), 75-125 $18 \mathrm{~ms}$ (express saccades), 125-250 ms (regular-latency saccades). The inset 19 plots the pooled saccade latency distribution. Figure conventions as in Figure $202 \mathrm{~A}$, except that non-overlapping $50 \mathrm{~ms}$ time-bins were used.

Figure 2 - figure supplement 1. Individual subjects - rapid post-saccadic recovery of performance. Data from the eight individual subjects whose pooled data appears in Figure 2A. Triangles indicate time at which performance reaches $80 \%$ of baseline (see Supplementary Experimental

26 Procedures); the values of this time are 24, 24, 23, 19, 24, 22, 23 and $35 \mathrm{~ms}$ 
1 for Subjects BA, JV, JS, JK, KW, LV, MK and SP respectively. Data plotted

2 using 20 ms time-bins. All other conventions as in Figure 2A. Related to

3 Figure 2A.

5 Figure 2 - figure supplement 2. Individual subjects - rapid post-saccadic

6 recovery of performance for two task difficulties. Data from the five

7 individual subjects whose pooled data appears in Fig.2B. Triangles indicate

8 time at which performance reaches $80 \%$ of baseline (see Supplementary

9 Experimental Procedures); the values of this time are 25, 24, 29, 30, and 25

$10 \mathrm{~ms}$ for the easier task and $29,40,32,30$, and $21 \mathrm{~ms}$ for the harder task for

11 subjects JV, LV, MK, MS and TY respectively. All other conventions as in

12 Figure 2B. Related to Figure 2B.

14 Figure 2 - figure supplement 3. Results from experiment 3, where distractor changes are more numerous and more salient also show 16 rapid post-saccadic recovery of performance (within $30 \mathrm{~ms}$ ), and no 17 evidence for post-saccadic retinotopic persistence or pre-saccadic 18 predictive shifts. Pooled data in A and data from individual subjects in B. 19 Triangles in B indicate time at which performance reaches $80 \%$ of baseline 20 (see Supplementary Experimental Procedures); the values of this time are $2128,35,30$, and $26 \mathrm{~ms}$ for subjects JV, JS, LV and MK respectively. All other 22 conventions as in Figure 2A. Related to Figure 2A.

Figure 2 - figure supplement 4. Post-saccadic recovery of performance 25 plotted relative to saccade onset. This figure is identical to Figure 2, 26 except that the performance (in Experiments 1 and 2 ) is plotted as a function 
1 of the time of target change relative to saccade onset. Recovery times

2 relative to saccade onset are $60 \mathrm{~ms}$ in $A$ and $70 \mathrm{~ms}$ for both tasks in $B$.

3 Related to Figures $2 \mathrm{~A}$ and $2 \mathrm{~B}$.

4

5 Figure 2 - figure supplement 5. Post-saccadic recovery of performance

6 plotted with a smaller fixation window. This figure is identical to Figure 2,

7 except that we only included trials where the horizontal and vertical eye-

8 positions did not diverge by more than 0.5 degrees during fixation from their

9 median values (see Methods). Estimated recovery times are $20 \mathrm{~ms}$ in A and

$1030 \mathrm{~ms}$ for both tasks in B. Despite the much smaller fixation window, $A$ and $B$

11 include $59 \%$ and $78 \%$ of the trials in Figures $2 \mathrm{~A}$ and $2 \mathrm{~B}$ respectively.

12 Related to Figures $2 \mathrm{~A}$ and $2 \mathrm{~B}$.

14 Supplementary File 1A: Experiments 1 and 2. False-positive rate from 0 to

$15150 \mathrm{~ms}$ after saccade offset shows no effect of post-saccadic retinotopic 16 attentional persistence.

17

18 Supplementary File 1B: The false-positive rate in Experiment 3 shows no 19 effect of either pre-saccadic predictive shifts or post-saccadic retinotopic 20 persistence. 


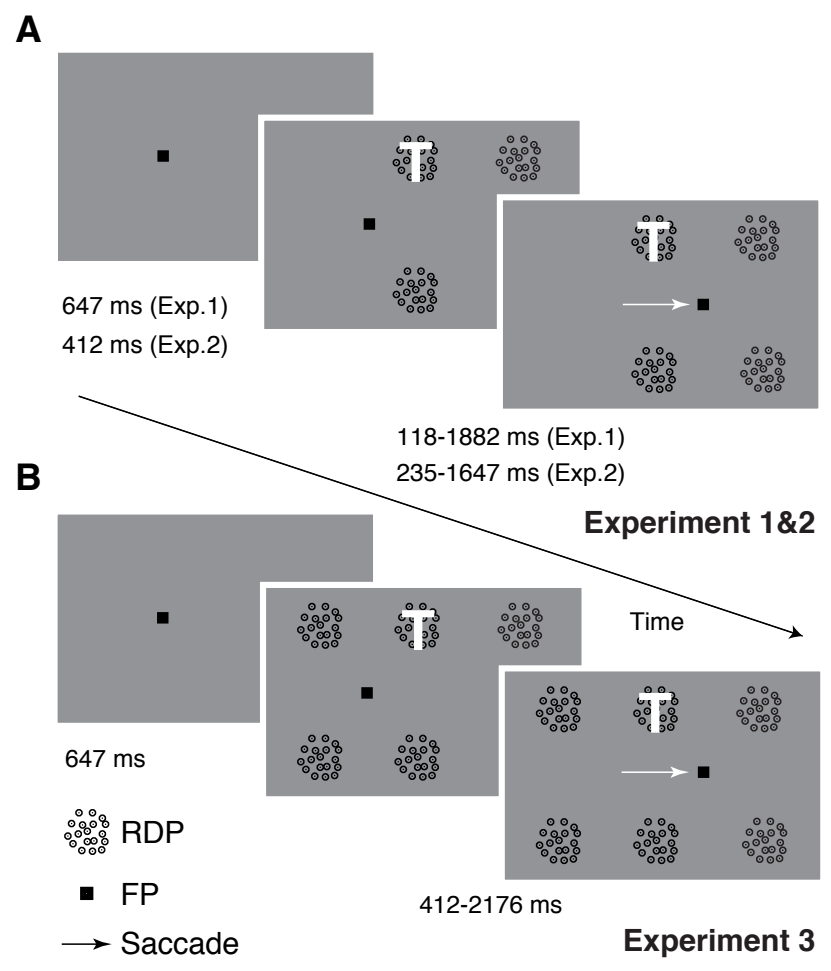

Figure 1. Task-design and timing. Human subjects performed a task that involved attending to a target (marked with a white $T$ and always at the same location) presented as one among four (A) or six (B) moving RDPs while also making a visually guided saccade if the fixation point jumped seven degrees to the right $(1235 \mathrm{~ms}$ after trial onset). The subjects were instructed to respond with a key-press when the target RDP briefly changed speed and direction, but to ignore similar changes in any of the remaining RDPs. Target and distractor changes occurred at different times around the saccade, enabling the measurement of peri-saccadic performance in this attention task. Two different task-difficulties were used in Experiment 2, while six RDPs were used in Experiment 3 instead of four. There were also minor differences in timing between the three tasks. 

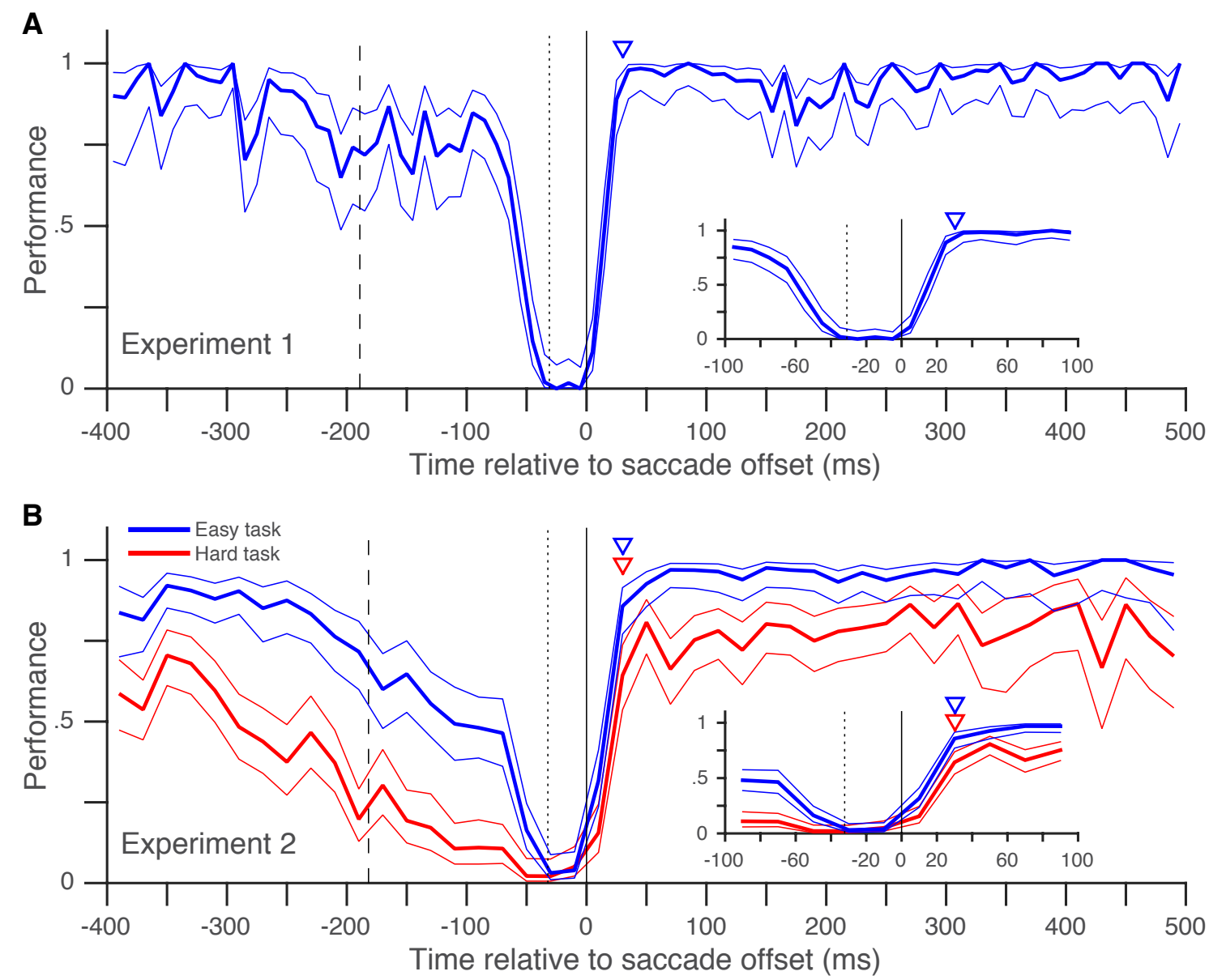

Figure 2. Rapid post-saccadic recovery of performance. A) Detection-performance (hit-rate) of motion-direction drops around the time of the saccade and recovers within $30 \mathrm{~ms}$ after the saccade. The figure shows the mean detection-performance (and $95 \%$ confidence bands) for all trials pooled over 8 subjects calculated in non-overlapping $10 \mathrm{~ms}$ time-bins of the abscissa (time of target-change relative to saccade offset). The inset shows the same data, focusing on the time between -100 and $100 \mathrm{~ms}$. Data from individual subjects show little inter-individual variability in the time-course of recovery (figure supplement 1). The triangle indicates the earliest time (30 ms) at which performance is statistically indistinguishable from that over the 100 to $500 \mathrm{~ms}$ timeperiod (using Boschloo's exact test; see Experimental Procedures). The dashed vertical line indicates the mean time of fixation-point offset and the stippled vertical line indicates the mean saccade onset time. See also figure supplements 1 and 3. B) Similar results were obtained when two different task-difficulties were used (data pooled over 5 subjects). The data from the higherdifficulty task (in red) show that the rapid recovery is not an artifact of a ceiling effect on performance. Data plotted using $20 \mathrm{~ms}$ time-bins. Figure conventions as in Figure $2 \mathrm{~A}$. See also figure supplement 2 for data from individual subjects. Figure supplements 4 and 5 replot the same data as in Figures $2 \mathrm{~A}$ and $2 \mathrm{~B}$ and in the same format, but figure supplement 4 uses the time of targetchange relative to saccade onset and figure supplement 5 only includes trials where a fixation window of 0.5 degrees was used (see corresponding legends for details). 


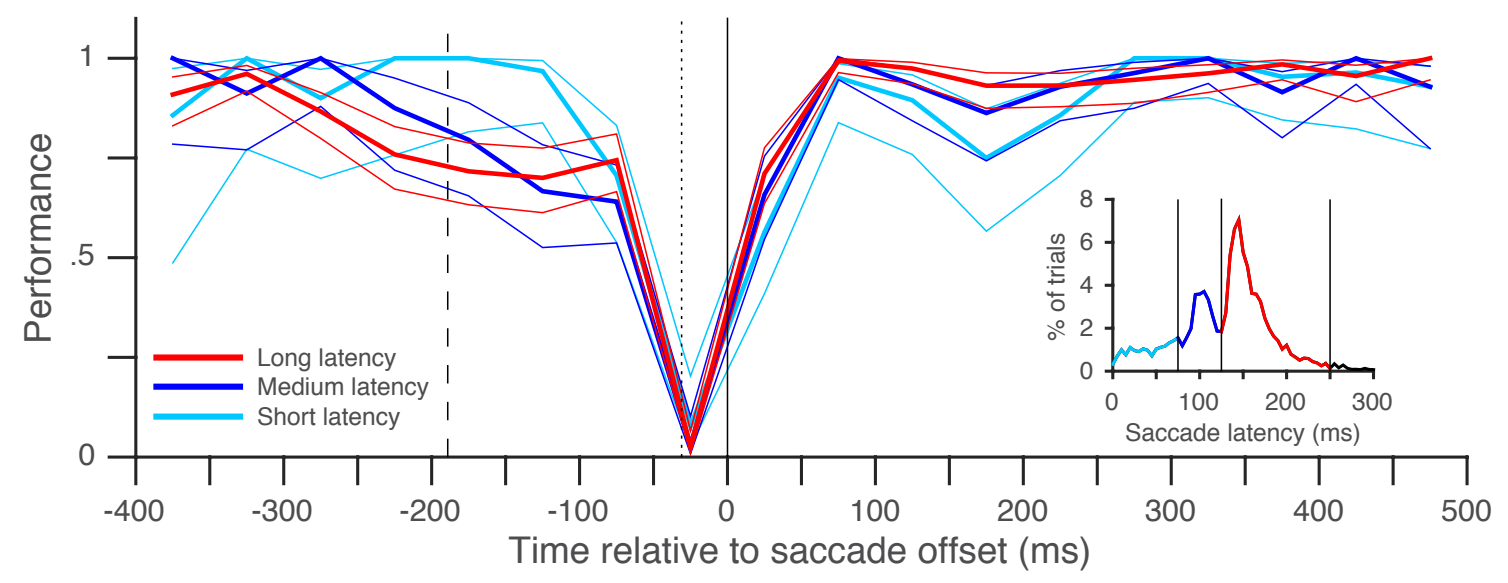

Figure 3. Rapid post-saccadic performance recovery is independent of saccade latency. The time-course of recovery was indistinguishable for saccades in three different latency ranges in the same dataset used in Figure 2A ( 8 subjects, color coding in inset): $0-75 \mathrm{~ms}$ (predictive saccades), $75-125 \mathrm{~ms}$ (express saccades), $125-250 \mathrm{~ms}$ (regular-latency saccades). The inset plots the pooled saccade latency distribution. Figure conventions as in Figure $2 \mathrm{~A}$, except that nonoverlapping $50 \mathrm{~ms}$ time-bins were used. 

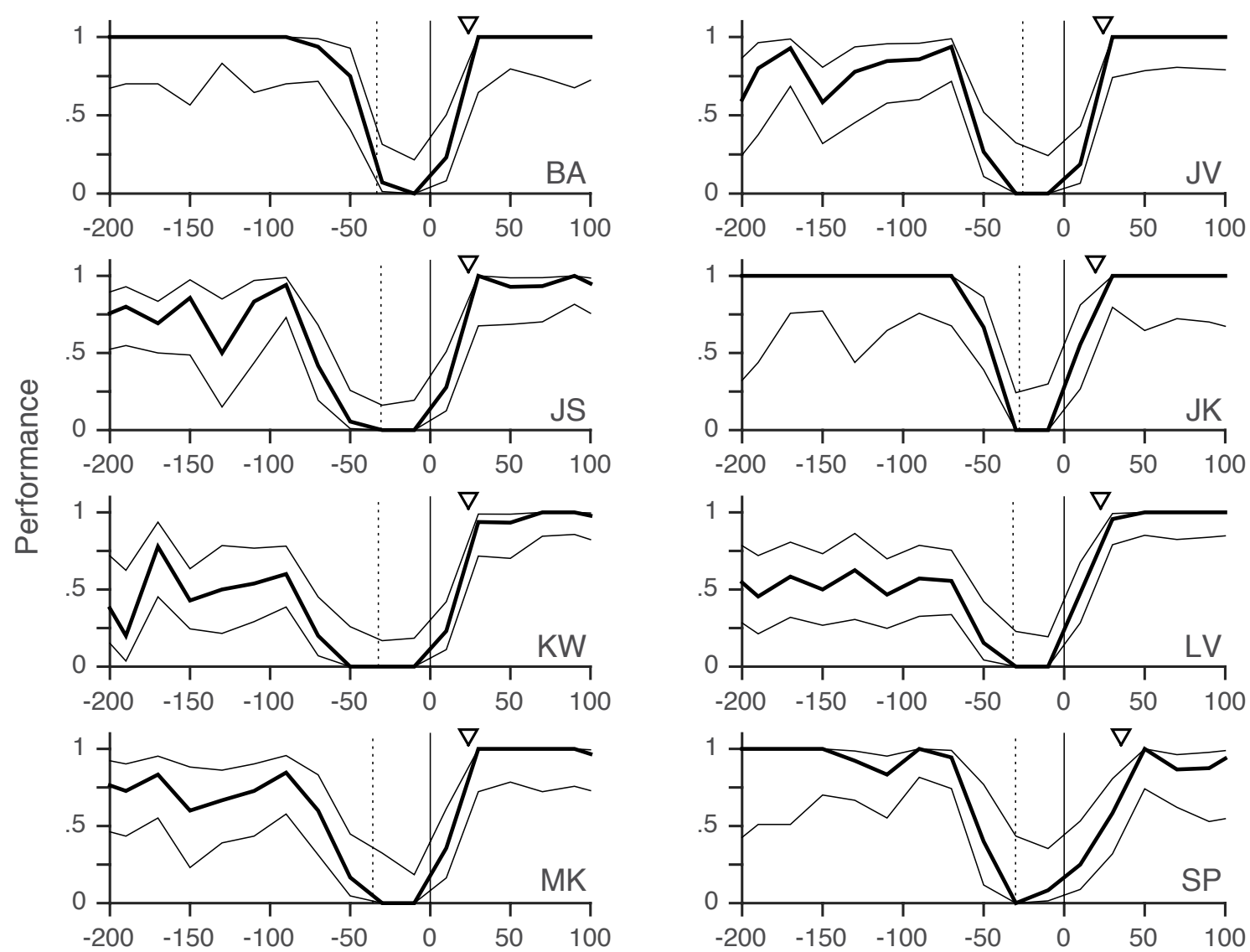

Time relative to saccade offset $(\mathrm{ms})$

Figure 2 - figure supplement 1. Individual subjects - rapid post-saccadic recovery of performance. Data from the eight individual subjects whose pooled data appears in Figure 2A. Triangles indicate time at which performance reaches $80 \%$ of baseline (see Supplementary Experimental Procedures); the values of this time are 24, 24, 23, 19, 24, 22, 23 and 35 ms for Subjects BA, JV, JS, JK, KW, LV, MK and SP respectively. Data plotted using $20 \mathrm{~ms}$ time-bins. All other conventions as in Figure 2A. Related to Figure 2A. 

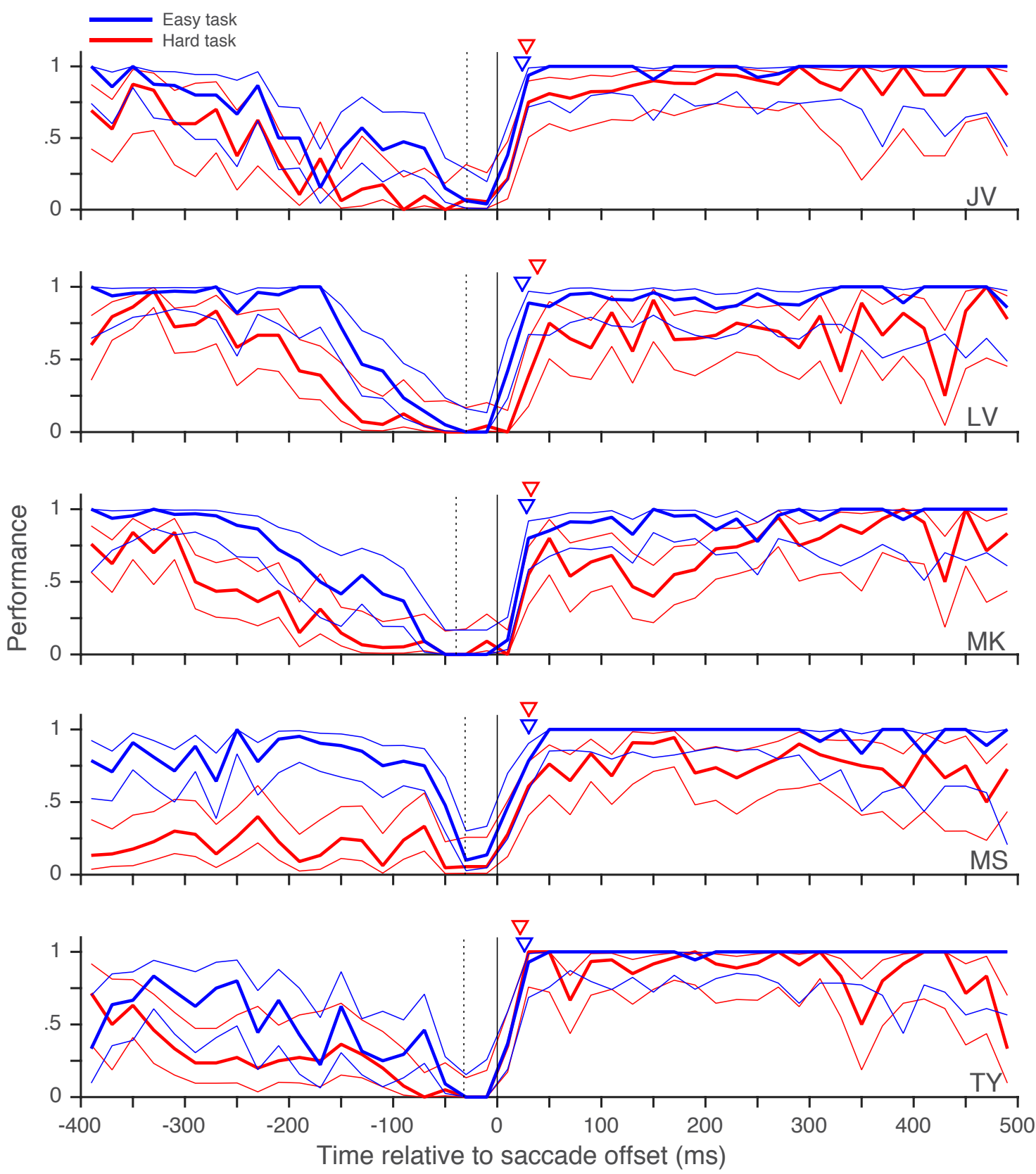

Figure 2 - figure supplement 2. Individual subjects - rapid post-saccadic recovery of performance for two task difficulties. Data from the five individual subjects whose pooled data appears in Fig.2B. Triangles indicate time at which performance reaches $80 \%$ of baseline (see Supplementary Experimental Procedures); the values of this time are 25, 24, 29, 30, and 25 ms for the easier task and 29, 40, 32, 30, and $21 \mathrm{~ms}$ for the harder task for subjects JV, LV, MK, MS and TY respectively. All other conventions as in Figure 2B. Related to Figure 2B. 
A

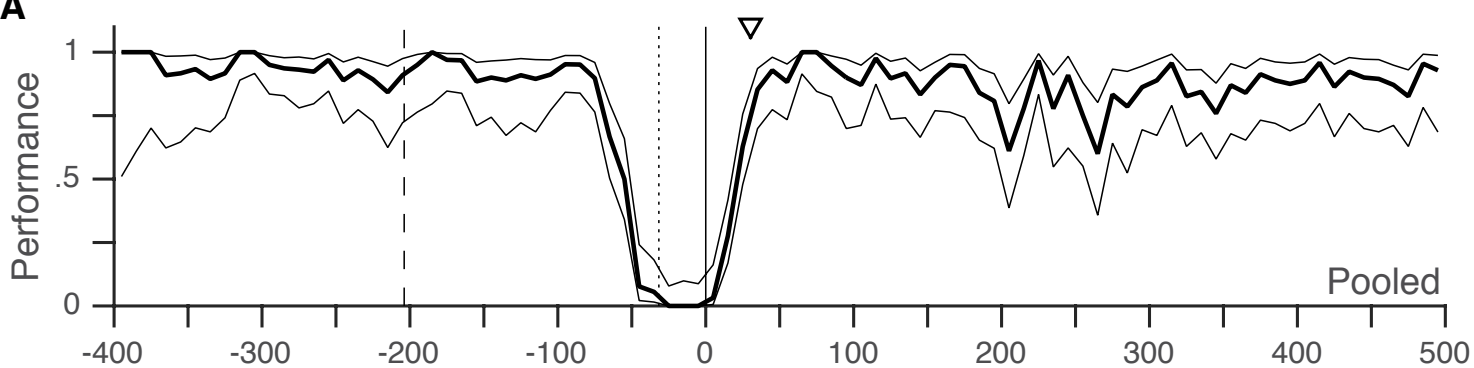

B
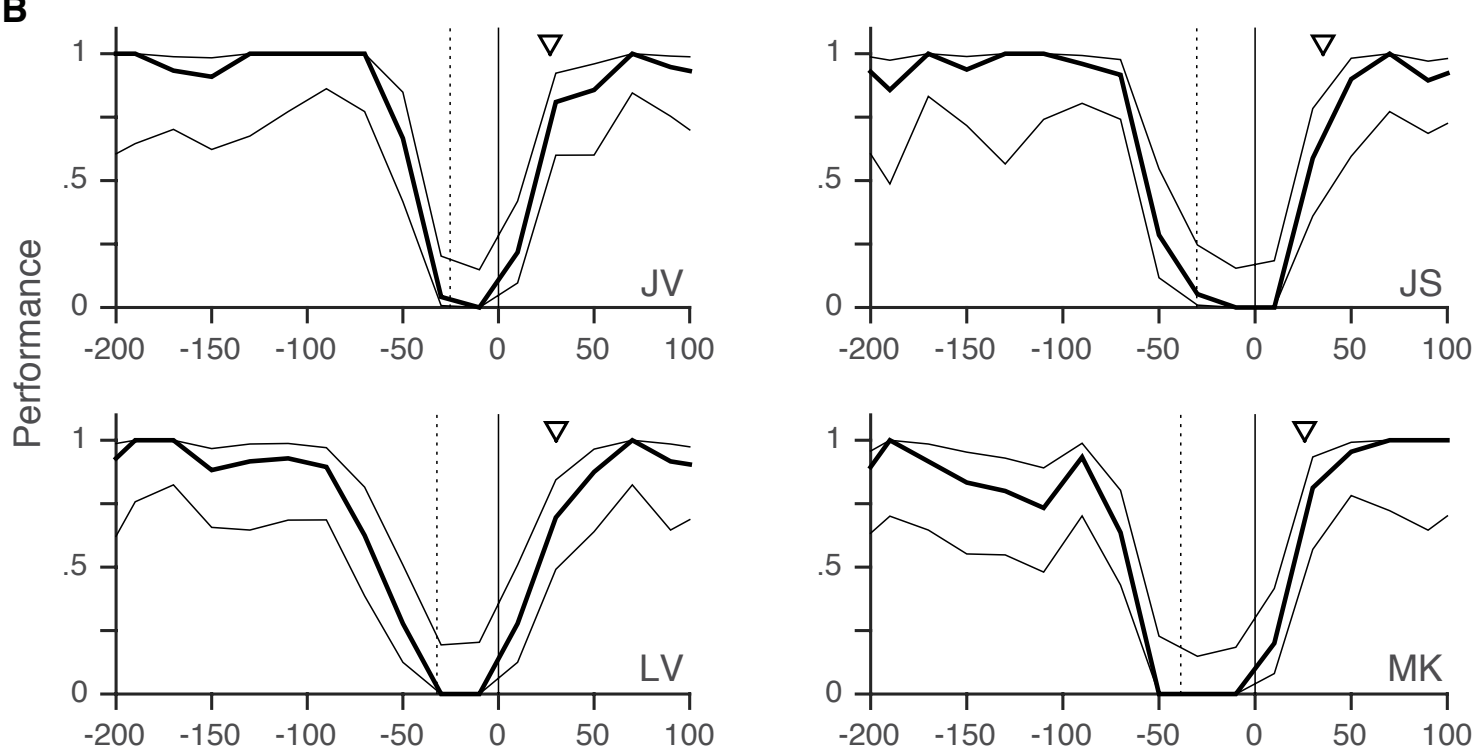

Time relative to saccade offset $(\mathrm{ms})$

Figure 2 - figure supplement 3. Results from experiment 3, where distractor changes are more numerous and more salient also show rapid post-saccadic recovery of performance (within $30 \mathrm{~ms}$ ), and no evidence for post-saccadic retinotopic persistence or pre-saccadic predictive shifts. Pooled data in A and data from individual subjects in B. Triangles in B indicate time at which performance reaches $80 \%$ of baseline (see Supplementary Experimental Procedures); the values of this time are 28, 35, 30, and 26 ms for subjects JV, JS, LV and MK respectively. All other conventions as in Figure 2A. Related to Figure 2A. 

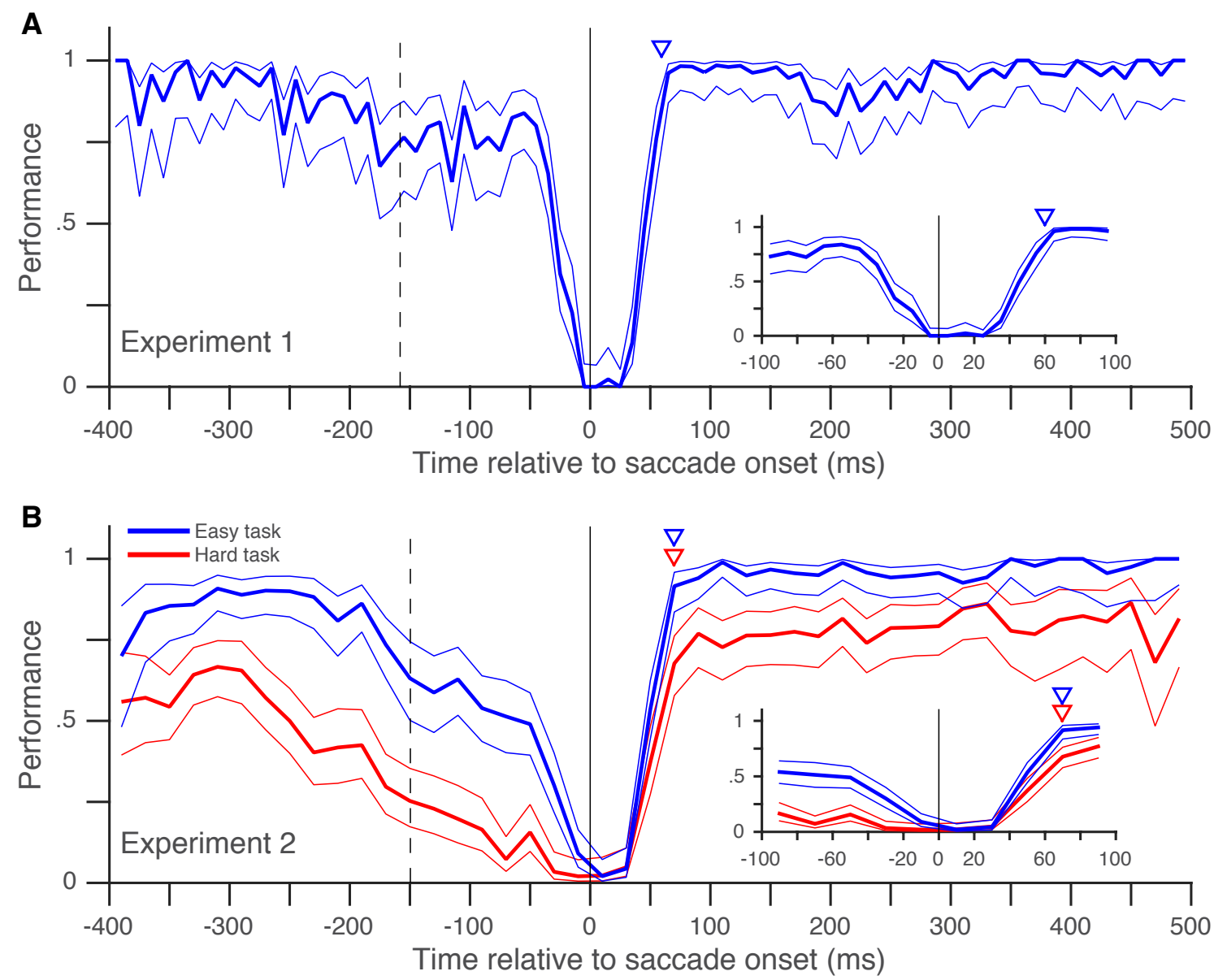

Figure 2 - figure supplement 4. Post-saccadic recovery of performance plotted relative to saccade onset. This figure is identical to Figure 2, except that the performance (in Experiments 1 and 2 ) is plotted as a function of the time of target change relative to saccade onset. Recovery times relative to saccade onset are $60 \mathrm{~ms}$ in $\mathrm{A}$ and $70 \mathrm{~ms}$ for both tasks in $\mathrm{B}$. Related to Figures $2 \mathrm{~A}$ and 2B. 
A
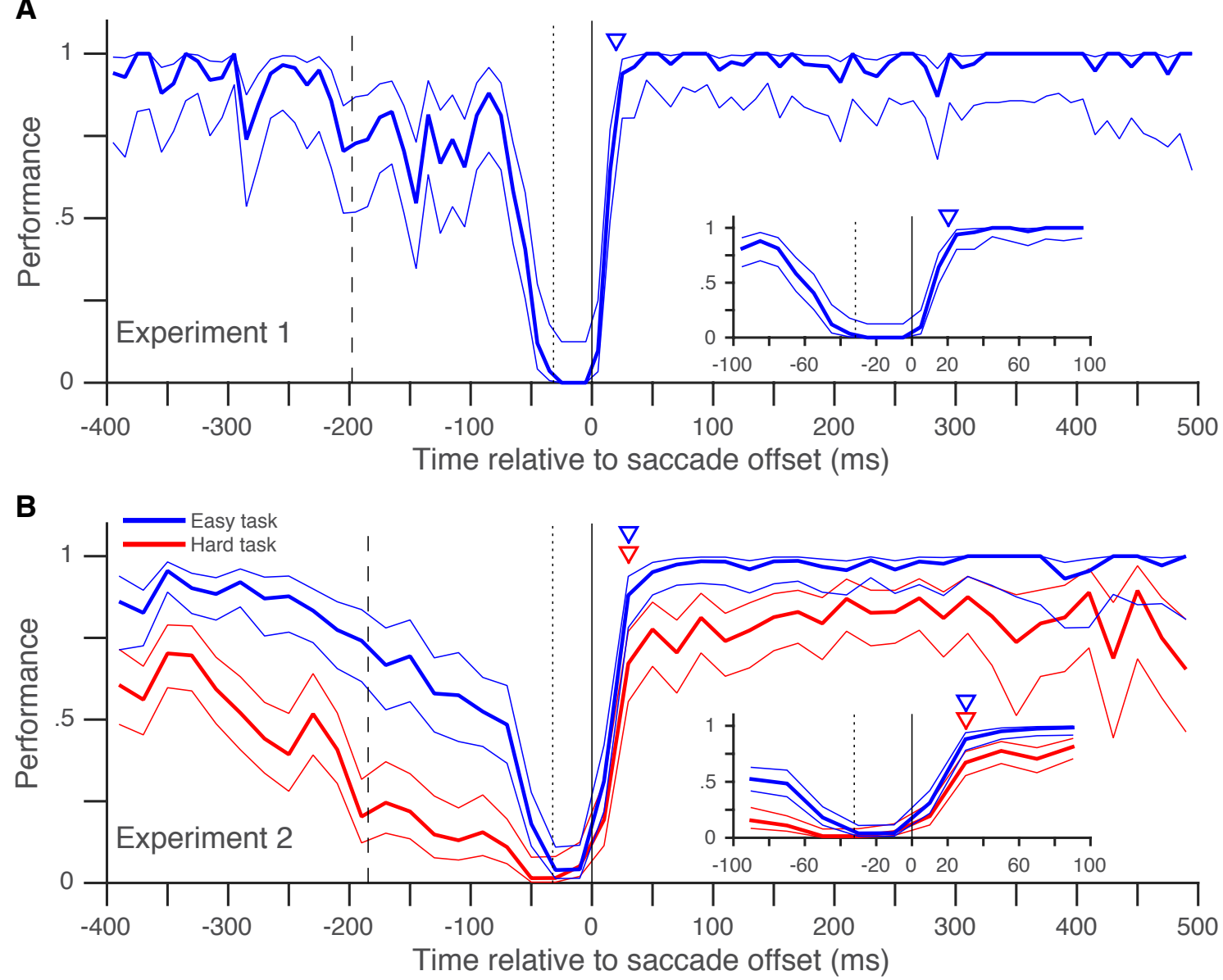

Figure 2 - figure supplement 5. Post-saccadic recovery of performance plotted with a smaller fixation window. This figure is identical to Figure 2, except that we only included trials where the horizontal and vertical eye-positions did not diverge by more than 0.5 degrees during fixation from their median values (see Methods). Estimated recovery times are $20 \mathrm{~ms}$ in A and 30 ms for both tasks in B. Despite the much smaller fixation window, A and B include $59 \%$ and $78 \%$ of the trials in Figures $2 \mathrm{~A}$ and $2 \mathrm{~B}$ respectively. Related to Figures $2 \mathrm{~A}$ and $2 \mathrm{~B}$. 
Supplementary File 1A: Experiments 1 and 2. False-positive rate from 0 to 150 ms after saccade offset shows no effect of post-saccadic retinotopic attentional persistence.

\begin{tabular}{|c|c|c|c|c|}
\hline & \multicolumn{2}{|c|}{$\begin{array}{c}\text { Distractor to right of target } \\
\text { (retinotopic persistence) }\end{array}$} & \multicolumn{2}{|c|}{$\begin{array}{c}\text { Distractor below target } \\
\text { (control) }\end{array}$} \\
\hline & No. False-positives & Total trials & No. False-positives & Total trials \\
\hline \multicolumn{5}{|c|}{ Experiment 1} \\
\hline Subject BA & 0 & 16 & 1 & 9 \\
\hline Subject JV & 0 & 25 & 0 & 12 \\
\hline Subject JS & 0 & 18 & 0 & 15 \\
\hline Subject JK & 0 & 17 & 0 & 17 \\
\hline Subject KW & 1 & 27 & 3 & 21 \\
\hline Subject LV & 0 & 26 & 0 & 23 \\
\hline Subject MK & 0 & 10 & 0 & 12 \\
\hline Subject SP & 4 & 24 & 1 & 18 \\
\hline Total & 5 & 163 & 5 & 127 \\
\hline \multicolumn{5}{|c|}{ Experiment 2} \\
\hline Subject JV & 1 & 14 & 5 & 14 \\
\hline Subject LV & 0 & 25 & 0 & 27 \\
\hline Subject MK & 0 & 15 & 0 & 17 \\
\hline Subject MS & 6 & 43 & 5 & 61 \\
\hline Subject TY & 2 & 18 & 0 & 17 \\
\hline Total & 9 & 115 & 10 & 136 \\
\hline
\end{tabular}


Supplementary File 1B: The false-positive rate in Experiment 3 shows no effect of either pre-saccadic predictive shifts or post-saccadic retinotopic persistence.

\begin{tabular}{|c|c|c|c|c|}
\hline & \multicolumn{2}{|c|}{$\begin{array}{c}\text { Distractor to right of target } \\
\text { (post-saccadic retinotopic persistence) }\end{array}$} & \multicolumn{2}{|c|}{$\begin{array}{c}\text { Distractor to left of target } \\
\text { (pre-saccadic predictive remapping) }\end{array}$} \\
\hline & No. False-positives & Total trials & No. False-positives & Total trials \\
\hline \multicolumn{5}{|c|}{ Pre-saccadic (200 to $0 \mathrm{~ms}$ before saccade offset) } \\
\hline Subject JV & 1 & 149 & 1 & 132 \\
\hline Subject JS & 0 & 158 & 2 & 155 \\
\hline Subject LV & 2 & 147 & 1 & 142 \\
\hline Subject MK & 1 & 125 & 1 & 120 \\
\hline Total & 4 & 579 & 5 & 549 \\
\hline \multicolumn{5}{|c|}{ Post-saccadic ( 0 to $150 \mathrm{~ms}$ after saccade offset) } \\
\hline Subject JV & 1 & 68 & 1 & 67 \\
\hline Subject JS & 0 & 66 & 0 & 87 \\
\hline Subject LV & 0 & 66 & 0 & 69 \\
\hline Subject MK & 0 & 55 & 3 & 68 \\
\hline Total & 1 & 255 & 4 & 291 \\
\hline
\end{tabular}




\section{Additional supplementary materials 1: Example eye traces}

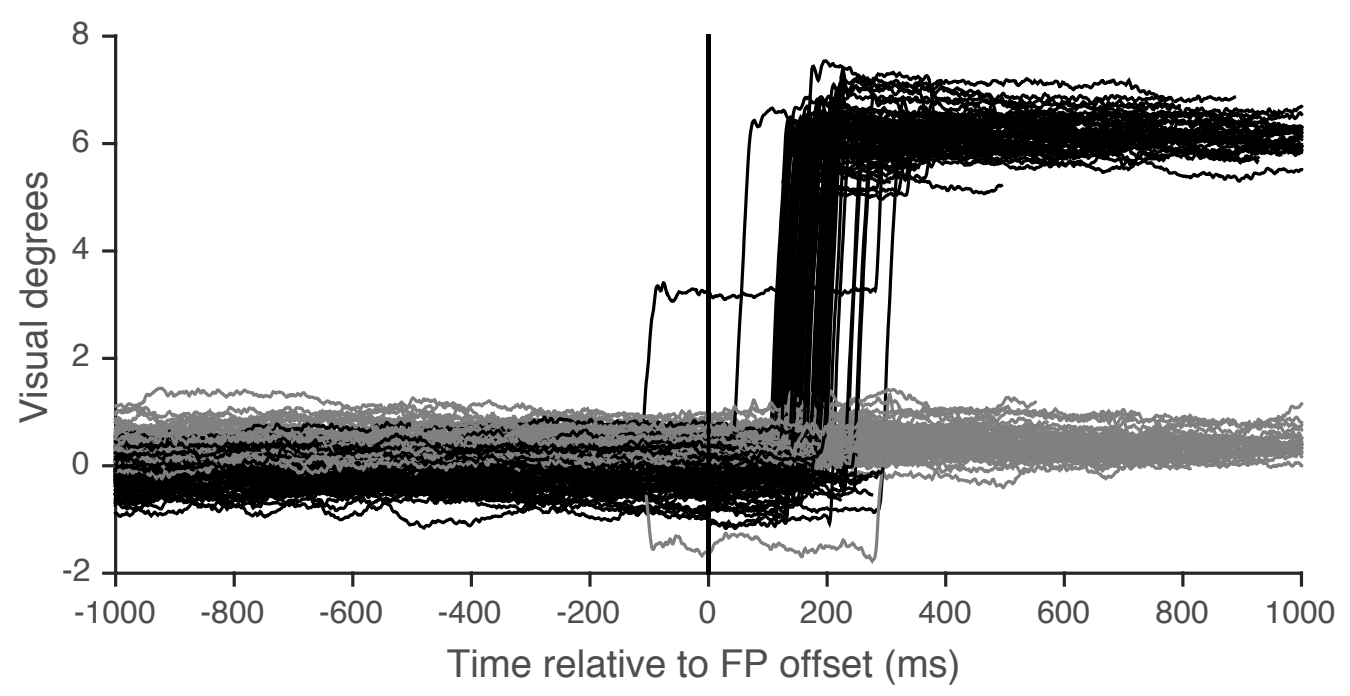

The example eye traces of trial No. 600-700 from subject BA in experiment 1 when he was performing the experiment and made a saccade in that trial. Not all of the trials showed here were included in further analysis (such as the one that the subject made a saccade before FP offset). The black and gray traces indicate the horizontal and vertical eye position respectively. In this experiment, the subject was asked to make a 7 visual angle degrees saccade to the right if he saw the fixation point jump. (the data from: mak-cha4s-bha-34567). 
Additional supplementary materials 2: Subjects information and data files

Subject information

\begin{tabular}{|c|c|c|c|c|}
\hline Three-letter code & Vision* & Age (years) & Gender & Handedness $^{* *}$ \\
\hline $\mathrm{BHA}$ & $\begin{array}{c}\text { Corrected to } \\
\text { normal }\end{array}$ & 25 & Male & Right handed \\
\hline JEV & Normal & 30 & Female & Right handed \\
\hline JUK & Normal & 21 & Female & Right handed \\
\hline JIS & $\begin{array}{c}\text { Corrected to } \\
\text { normal }\end{array}$ & 22 & Female & Right handed \\
\hline KAW & $\begin{array}{c}\text { Corrected to } \\
\text { normal }\end{array}$ & 22 & Male & Right handed \\
\hline LEV & Normal & 23 & Female & Right handed \\
\hline MAK & Normal & 22 & Female & Right handed \\
\hline MSS & Normal & 22 & Male & Right handed \\
\hline SNP & $\begin{array}{c}\text { Corrected to } \\
\text { Normal }\end{array}$ & 26 & Female & Right handed \\
\hline TAO & Normal & 29 & Male & Right handed \\
\hline
\end{tabular}

Table 1: The basic information of the subjects participating into this study.

*: If the subjects wear glasses, their vision was 'corrected to normal'; otherwise, their vision was 'normal'.

**: The handedness defined here as the subjects use which hand to write. 


\section{Maclab data files}

Note: In most of the cases, each file in the following pages was the data from one session, the length of each session could be different for each subjects. But, for some subjects, one subject could have less data files than others because the experimenter might only pause the experiment rather than terminated the experiment by exiting maclab when the subjects had a rest. The experimenter could also change the parameter after several sessions, which might cause the name of the first data file included in analysis with a larger numbers (such as LEV in experiment 1 and 2). The number in the data file names could be incontinuous because of the crash of maclab or other technical problems.

The first three letters in each data file name indicate the 3-letter code of the experimenter, follow the name of the epar running the experiment, then the 3-letter code of the subjects, then the number of the session.

\section{Experiment 1}

mak- cha4s -bha-003; mak- cha4s -bha-004; mak- cha4s -bha-005; mak- cha4s -bha-006; mak- cha4s -bha-007; mak- cha4s -jev-001; mak- cha4s -jev-002; mak- cha4s -jev-003; mak- cha4s -jev-004; mak- cha4s -jev-005; mak- cha4s -jev-006; mak- cha4s -juk-001; mak- cha4s -juk-002; mak- cha4s -juk-003; mak- cha4s -juk-004; mak- cha4s -juk-005; mak- cha4s -juk-006; mak- cha4s -juk-007; mak- cha4s -juk-008; mak- cha4s -jis-005; mak- cha4s - jis -006; mak- cha4s - jis -007; mak- cha4s - jis -008; mak- cha4s - kaw -001; mak- cha4s - kaw -002; mak- cha4s - kaw -003; mak- cha4s - kaw -004; mak- cha4s - kaw -005;

mak- cha4s - kaw -006;

mak- cha4s - kaw -007;

mak- cha4s - lev -007;

mak- cha4s - lev -008;

mak- cha4s - lev -010;

mak- cha4s - lev -011;

mak- cha4s - lev -012;

mak- cha4s - lev -013;

mak- cha4s - mak -002;

mak- cha4s - snp -001;

mak- cha4s - snp -002;

mak- cha4s - snp -003;

mak- cha4s - snp -004;

mak- cha4s - snp -005;

mak- cha4s - snp -006;

mak- cha4s - snp -007;

mak- cha4s - snp -008;

\section{Experiment 2}

mak- attdif -jev-001;

mak- attdif -jev-003;

mak- attdif -jev-004;

mak- attdif -lev-004;

mak- attdif -lev-005;

mak- attdif -lev-006;

mak- attdif -lev-007;

mak- attdif -mak-001;

mak- attdif -mak-002;

mak- attdif - mss -001;

mak- attdif - mss -002;

mak- attdif - mss -003;

mak- attdif - mss -004;

mak- attdif - mss -005;

mak- attdif - mss -006;

mak-attdif-tao-0210;

mak-attdif-tao-0218;

mak-attdif-tao-0218-002;

\section{Experiment 3}

mak-cha6s-jev-002;

mak-cha6s-jis-002;

mak-cha6s-jis-003;

mak-cha6s-lev-001;

mak-cha6s-lev-002;

mak-cha6s-mak-001; 


\section{Information for subjects participating in psychophysical tests in the Cognitive Neuroscience Laboratory, German Primate Center (DPZ)}

Name of subject:

Project leader:

Cognitive Neuroscience Laboratory, DPZ

I. Subject requirements

Participation in the tests requires normal or corrected-to-normal vision, and unrestricted arm- and hand-mobility.

\section{Aim of the study and benefit for the subject}

You participate voluntarily in this study. The purpose of the study is an improved knowledge of human perception and behavior. With your participation you contribute to our understanding of brain functions, especially of perception, the planning of movement, and the role of selective attention. We investigate how the efficiency of the sensorimotor system is influenced by prior knowledge and by the exact sensory circumstances of a particular experimental situation. For example, your performance will be compared between tasks, which differ only in the focus of your visual attention. Differences in performance between these task conditions allow conclusions about the influence of selective attention to the processing of sensory information.

A detailed understanding of the function of the healthy organism and brain is an important prerequisite for helping patients suffering from specific visual or motor deficits. The participation in the tests per se does not yield any direct health benefit.

\section{Design of the study}

You will participate in measurements, in which we will test your sensory or sensorimotor performance. For this, a number of different stimuli will be presented in random order on a computer screen or with a tactile stimulator. You will sit on a chair in front of these devices, sometimes with your chin on a chin rest in order to guarantee a defined distance to the monitor. You will respond by pressing a button on a computer keyboard, by touching a touch-screen, by making an eye movement or by pressing a footswitch (for simplicity we only speak of 'keystroke' in the following). A test consists of several trials. In each trial one or more visual stimuli will be presented. At the end of each trial you will respond with a keystroke. For example, the stimulus could consist of a moving pattern and you will have to decide if it is moving to the left or to the right. For some tests, it is important, that you change your direction of gaze as little as possible. For those tests, you will have to maintain your gaze on a small stimulus on the screen during the trials.

\section{Procedure}

A single test normally consists of 50 to 200 trials of a few seconds duration each. A test will be finished after 5 to 20 minutes. During one session several tests and different tasks will be conducted. Before each new task you will have opportunity to practice. A session typically takes one hour, including breaks between the tests. You will set the pace as in most measurements you start every trial yourself and because you can take breaks whenever you choose. There are also breaks between the tests and the task of the next test will be explained. Typically, a study consists of several sessions and the first sessions are used for training. It is very important for us, that you finish a study completely. But 
you are free to abort the measurements at any time.

\section{Side effects}

These tests are absolutely non-invasive experiments. There are no adverse effects. The computers and screens in use are standard equipment as used in offices and for computer games.

\section{Voluntariness of participation}

The participation in the study is absolutely voluntary. You are entitled to quit the study at any time and without giving reasons.

\section{Payment}

For the participation in the measurements you receive a payment. In some of our studies we investigate the influence of such payments on the performance of the subjects. In these sessions a basic payment is combined with an amount that depends on the psychophysical performance or is chosen at random.

\section{Passing on of the data}

The data collected in these studies will only be used or passed on anonymously.

\section{Consent form:}

I read and understood this information carefully. The experimenter answered all my questions. By signing this form I agree to

a) participate in the study and

b) to the publication of the collected data in an anonymous form.

I received a copy of this information sheet.

Place and date

Subject's signature

Cognitive Neuroscience Laboratory

German Primate Center - Leibniz Institute for Primate Research

Prof. Dr. Stefan Treue

Kellnerweg 4, 37077 Goettingen

Tel: 0551-3851-118

Vorsitzender des Aufsichtsrates: MR Dr. Axel Kollatschny;

Geschäftsführer: Prof. Dr. Stefan Treue, Assessor jur. Michael Lankeit;

Sitz der Gesellschaft: Göttingen; Handelsregister: Göttingen HRB 933 
Additional supplementary materials 4:

Example epars \& mpars

Note: The epars define the time sequence of the experiment, the location of the stimulus, i.e. when and where to show the stimulus on the screen. The epars also on-line control the data collection and some events related to subject performance (such as eye position, time window of reaction etc.). The mpars controls the properties of the stimuli, such as the motion direction, speed, dot density, color of the RDP.

\section{Example epars: \\ Experiment 1 \\ HuAtt4S.epar}

projectLeader mak

expCode cha4s

displayWindowOnSecondMonitor 00200200

trialProtocol 17

000010

100000

10600011

$\begin{array}{llllll}55 & 0 & 140 & -160 & 1 & 0\end{array}$

$\begin{array}{llllll}55 & 0 & 140 & 160 & 1 & 0\end{array}$

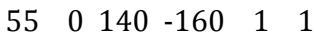

$\begin{array}{llllll}55 & 0 & 140 & 160 & 1 & 1\end{array}$

\# 2 stimuli in FRF \# 3 cue in FRF;

\# 4 1st FP; 5 patch opposite to FRF; 6 cue for $5 ; 7$

2nd FP or saccade target;

\# 8 and 9 patches removed before saccade in FRF;

\# 10 and 11 patches in RF; 12 and 13 patches removed before saccade in $\mathrm{RF}$.

\# NOTE this epar is used to training only, 4

patches would change directions

$\begin{array}{llll}\text { displayCenter } 0 & 1 & 0.4 & 0.5\end{array}$

displayCenter $1 \quad 1280 \quad 0$

filenames\&pathname 7 :cha4sSlow:traceMpars:

Luminance.mpar

fixStim4.mpar

fixStim6.mpar

Att4TP.mpar

Att4DP.mpar

Att4DP1.mpar

Att4TPL.mpar

\# 1 target +3 distractors

\# class 1 , catch trials, no change for target, 3 times

less than other classes

\# class 2, target in upper visual field, no changedistractor.

\# class3, target in upper visual field, change-

distractor in lower.
\# class 4 , target in upper visual field,changedistractor in future RF.

classes 4

12036666101

12034666201

12037566301

12037656401

classFrequencyOption 2

classFrequency 23

classFrequency 33

classFrequency 43

keyboardResponse TRUE

allowedFinalResponseKeys 1125

anticipatedResponseDuration 150

responseTimeWindow 600

fixpointIndexColor 255

backgroundIndexColor 100

fixPointSize 12

earlyResponsePermitted FALSE

fixPointVisibleBetweenTrials false

fixationRequired FALSE

collectEyePositions TRUE

fixAreaRadiusOfClass 080

smoothEyePositions 4

sampleSource 1

collectSpikes FALSE

numberOfTrials 9000

dotPosToBuffer FALSE

eyePosDecay -20

provideWords TRUE

openDataFile

savePupilDiameter TRUE

minPhaseChangeDistance 34

evaHack TRUE

leverDownRewardDuration 0

leverReleaseDuration 600

\section{Example mpars:}

\section{Luminance.mpar}

$\begin{array}{llll}\text { numberOfSurfaces } & 1 & 0 & 0\end{array}$

$\begin{array}{ll}\text { pixelradius } \quad 8 & \\ \text { numberOfFrames } & 300\end{array}$

qtRGBForeColor $65000 \quad 65000 \quad 65000$

$\begin{array}{lllll}\text { qtSquare } & -6 & -6 & 6 & 6\end{array}$

changePhase $\quad-150 \quad 250$

qtRGBForeColor $35000 \quad 35000 \quad 35000$

$\begin{array}{lllll}\text { qtSquare } & -6 & -6 & 6 & 6\end{array}$

changePhase $10 \quad 10$

qtRGBForeColor $65000 \quad 65000 \quad 65000$

$\begin{array}{lllll}\text { qtSquare } & -6 & -6 & 6 & 6\end{array}$ 


\section{FixStim4.mpar}

numberOfSurfaces 1400

pixelradius $\quad 10$

numberOfFrames 105

\#qtRGBForeColor 655365530

qtIndexForeColor 255

$\begin{array}{llllll}\text { qtSquare } & -6 & -6 & 6 & 6\end{array}$

qtSuspendFix 103152

qtFixFactor

$105-1 \quad 1.5$

qtFixDispCtr $\quad 105-1 \quad 1$

\section{Fixstim6.mpar}

numberOfSurfaces 1400

pixelradius 10

numberOfFrames 200

\#qtRGBForeColor

qtIndexForeColor 255

$\begin{array}{lllll}\text { qtSquare } & -6 & -6 & 6 & 6\end{array}$

qtSuspendFix 103152

qtFixFactor $\quad 105-1 \quad 1.5$

qtFixDispCtr $105-1 \quad 1$

\section{Att4TP.mpar}

numberOfSurfaces 14010

dotSize 6

pixelradius 80

numberOfFrames 250

speed 4

shape circle

colorIndex 255

direction 0

changePhase -20 100

directionTable 290270

speed 32

changePhase 22

direction 0

speed 4

\section{Att4DP.mpar}

numberOfSurfaces 14010

dotSize 6

pixelradius 80

numberOfFrames 250

speed 4

shape circle

colorIndex 255

direction 0

changePhase 10160

directionTable 290270

speed 32

changePhase 22

direction 0

speed 4

\section{Att4DP1.mpar}

numberOfSurfaces 14010

dotSize 6

pixelradius 80

numberOfFrames 250

speed 4

shape circle

colorIndex 255

direction 0

\section{Att4TPL.mpar}

[numberOfSurfaces 14010

dotSize 6

pixelradius 80

numberOfFrames 250

speed 4

shape circle

colorIndex 255

direction 0

changePhase -10 160

directionTable 290270

speed 32

changePhase 22

direction 0

speed 4

\section{Experiment 2}

\section{Attdif.epar}

projectLeader mak

expCode AttDif

displayWindowOnSecondMonitor 00200200

trialProtocol 17

000010

100010

10600011

$\begin{array}{llllll}35 & 0 & 140 & -160 & 1 & 0\end{array}$

$\begin{array}{llllll}35 & 0 & 140 & 160 & 1 & 0\end{array}$

$\begin{array}{llllll}35 & 0 & 140 & -160 & 1 & 1\end{array}$

$\begin{array}{llllll}35 & 0 & 140 & 160 & 1 & 1\end{array}$

\# 2 stimuli in FRF \# 3 cue in FRF;

\# 4 1st FP; 5 patch opposite to FRF; 6 cue for $5 ; 7$

2nd FP or saccade target;

\# 8 and 9 patches removed before saccade in FRF;

\# 10 and 11 patches in RF; 12 and 13 patches

removed before saccade in $\mathrm{RF}$.

\# NOTE this epar is used to training only, 4

patches would change directions

displayCenter $0 \quad 1 \quad 0.4 \quad 0.5$

displayCenter $1 \quad 1280 \quad 0$

filenames\&pathname 13 :AttDiff:traceMpars:

Luminance.mpar

fixStim4.mpar

fixStim6.mpar 


\author{
Att4TP.mpar \\ Att4DP.mpar \\ Att4DP1.mpar \\ Att4TPL.mpar \\ Att4TP40.mpar \\ Att4DP40.mpar \\ Att4TPL40.mpar \\ Att4TP55.mpar \\ Att4DP55.mpar \\ Att4TPL55.mpar \\ \# 1 target +3 distractors \\ \# class 1, catch trials, no change for target, 3 times \\ less than other classes \\ \# class 2,5, target in upper visual field, no change- \\ distractor. \\ \# class 3,6, target in upper visual field.change- \\ distractor in lower. \\ \# class 4,7, target in upper visual field,change- \\ distractor in future RF.
}

\section{classes 7}

12036666101

12038666201

120310966301

120310696401

120311666501

1203131266601

1203136126701

classFrequencyOption 2

classFrequency 54

classFrequency 24

classFrequency 32

classFrequency 42

classFrequency 62

classFrequency 72

keyboardResponse TRUE

allowedFinalResponseKeys 1125

anticipatedResponseDuration 150

responseTimeWindow 600

fixpointIndexColor 255

backgroundIndexColor 100

fixPointSize 12

earlyResponsePermitted FALSE

fixPointVisibleBetweenTrials FALSE

fixationRequired FALSE

collectEyePositions TRUE

fixAreaRadiusOfClass 080

smoothEyePositions 4

sampleSource 1

collectSpikes FALSE

numberOfTrials 9000

dotPosToBuffer FALSE

eyePosDecay -20

provideWords TRUE
openDataFile

savePupilDiameter TRUE

minPhaseChangeDistance 34

evaHack TRUE

leverDownRewardDuration 0

leverReleaseDuration 600

\section{Example mpars:}

\section{Att4TP40.mpar}

numberOfSurfaces 14010

dotSize 6

pixelradius 80

numberOfFrames 250

speed 8

shape circle

colorIndex 255

direction 0

changePhase -50 110

directionTable 240320

changePhase 22

direction 0

speed 8

\section{Att4DP40.mpar}

numberOfSurfaces 14010

dotSize 6

pixelradius 80

numberOfFrames 250

speed 8

shape circle

colorIndex 255

direction 0

changePhase 10160

directionTable 240320

changePhase 22

direction 0

speed 8

\section{Att4TPL40.mpar}

numberOfSurfaces 14010

dotSize 6

pixelradius 80

numberOfFrames 250

speed 8

shape circle

colorIndex 255

direction 0

changePhase -20 140

directionTable 240320

changePhase 22

direction 0

speed 8

\section{Experiment 3}




\section{Cha6S.epar}

projectLeader mak

expCode cha6s

displayWindowOnSecondMonitor 00200200

trialProtocol 19

0000010

100000

10600011

$\begin{array}{llllll}55 & 0 & 140 & -160 & 1 & 0\end{array}$

$\begin{array}{llllll}55 & 0 & 140 & 160 & 1 & 0\end{array}$

$\begin{array}{llllll}55 & 0 & 140 & -160 & 1 & 1\end{array}$

$\begin{array}{llllll}55 & 0 & 140 & 160 & 1 & 1\end{array}$

$\begin{array}{llllll}55 & 0 & -140 & -160 & 1 & 0\end{array}$

$\begin{array}{llllll}55 & 0 & -140 & 160 & 1 & 0\end{array}$

\# 2 stimuli in FRF \# 3 cue in FRF;

\# 4 1st FP; 5 patch opposite to FRF; 6 cue for $5 ; 7$

2nd FP or saccade target;

\# 8 and 9 patches removed before saccade in FRF;

\# 10 and 11 patches in RF; 12 and 13 patches removed before saccade in $R F$.

\# NOTE this epar is used to training only, 4

patches would change directions

$\begin{array}{lllc}\text { displayCenter } 0 & 1 & 0.4 & 0.5 \\ \text { displayCenter } 1 & 1 & 280 & 0\end{array}$

filenames\&pathname 9 :cha6sSlow:traceMpars:

Luminance.mpar

fixStim4.mpar

fixStim6.mpar

Att4TP.mpar

Att4DP.mpar

Att4DP1.mpar

Att4TPL.mpar

Att4TPL2.mpar

Att4TPL0.mpar

\# 1 target +5 distractors

\# class 1 , catch trials, no change for target, 3 times less than other classes

\# class 2, target in upper visual field, no changedistractor

\# class 3, target in upper visual field.change-

distractor in left (remapping location).

\# class 4, target in upper visual field,change-

distractor in future RF.

classes 4

1203966666101

1203466666201

1203766656301

1203765666401

classFrequencyOption 2

classFrequency 25

classFrequency 34

classFrequency 44

keyboardResponse TRUE

allowedFinalResponseKeys 1125
anticipatedResponseDuration 150

responseTimeWindow 600

fixpointIndexColor 255

backgroundIndexColor 100

fixPointSize 12

earlyResponsePermitted FALSE

fixPointVisibleBetweenTrials false

fixationRequired FALSE

collectEyePositions TRUE

fixAreaRadiusOfClass 080

smoothEyePositions 4

sampleSource 1

collectSpikes FALSE

numberOfTrials 9000

dotPosToBuffer FALSE

eyePosDecay -20

provideWords TRUE

openDataFile

savePupilDiameter TRUE

minPhaseChangeDistance 34

evaHack TRUE

leverDownRewardDuration 0

leverReleaseDuration 600

\section{Example mpars:}

\section{fixStim4.mpar}

numberOfSurfaces 1400

pixelradius $\quad 10$

numberOfFrames 105

\#qtRGBForeColor

655365530

qtIndexForeColor 255

$\begin{array}{lll}\text { qtSquare } & -6 & -6 \\ \text { qtSuspendFix } & 103 & 152\end{array}$

qtFixFactor $\quad 105-1 \quad 1.5$

qtFixDispCtr $\quad 105-1 \quad 1$

\section{fixStim6.mpar}

numberOfSurfaces 1400

pixelradius 10

numberOfFrames 200

qtIndexForeColor 255

$\begin{array}{ll}\text { qtSquare } & -6 \\ \text { qtSuspendFix } & 103152\end{array}$

qtFixFactor $\quad 105-1 \quad 1.5$

qtFixDispCtr $\quad 105-1 \quad 1$

\section{Att4TP.mpar}

numberOfSurfaces 14010

dotSize 6

pixelradius 80

numberOfFrames 250

speed 4

shape circle

colorIndex 255 
direction 0

changePhase -35 75

directionTable 290270

speed 32

changePhase 22

direction 0

speed 4

\section{Att4DP.mpar}

numberOfSurfaces 14010

dotSize 6

pixelradius 80

numberOfFrames 250

speed 4

shape circle

colorIndex 255

direction 0

changePhase 4080

directionTable 290270

speed 32

changePhase 22

direction 0

speed 4

\section{Att4DP1.mpar}

numberOfSurfaces 14010

dotSize 6

pixelradius 80

numberOfFrames 250

speed 4

shape circle

colorIndex 255

direction 0

\section{Att4TPL.mpar}

numberOfSurfaces 14010

dotSize 6

pixelradius 80

numberOfFrames 300

speed 4

shape circle

colorIndex 255

direction 0

changePhase -75 185

directionTable 290270

speed 32

changePhase 22

direction 0

speed 4

\section{Att4TPL0.mpar}

numberOfSurfaces 14010

dotSize 6

pixelradius 80

numberOfFrames 300 speed 4

shape circle

colorIndex 255

direction 0

changePhase -40 185

directionTable 200

changePhase 22

direction 0

speed 4 
Chapter 4

An attention-sensitive memory trace in macaque MT following saccadic eye movements 


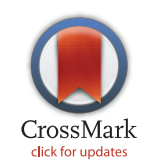

\section{G openaccess}

Citation: Yao T, Treue S, Krishna BS (2016) An Attention-Sensitive Memory Trace in Macaque MT Following Saccadic Eye Movements. PLoS Biol 14 (2): e1002390. doi:10.1371/journal.pbio.1002390

Academic Editor: Christopher C. Pack, McGill University, CANADA

Received: September 21, 2015

Accepted: January 26, 2016

Published: February 22, 2016

Copyright: @ 2016 Yao et al. This is an open access article distributed under the terms of the Creative Commons Attribution License, which permits unrestricted use, distribution, and reproduction in any medium, provided the original author and source are credited.

Data Availability Statement: All relevant data are within the paper and its Supporting Information files.

Funding: This work was supported by the Federal Ministry of Education and Research (BMBF),

Germany, under grant number 01GQ1005C; grants of the Deutsche Forschungsgemeinschaft through the Collaborative Research Center 889 "Cellular Mechanisms of Sensory Processing" to ST (Project C04), and by the Germany-Israeli Foundation for Scientific Research and Development Grant No. 1108-79.1/2010. The publication of this article was funded by the Open Access fund of the Leibniz Association.
RESEARCH ARTICLE

\section{An Attention-Sensitive Memory Trace in Macaque MT Following Saccadic Eye Movements}

\author{
Tao Yao $^{1 *}$, Stefan Treue ${ }^{1,2,3}$, B. Suresh Krishna ${ }^{1 *}$ \\ 1 Cognitive Neuroscience Laboratory, German Primate Center, Goettingen, Germany, 2 Bernstein Center \\ for Computational Neuroscience, Goettingen, Germany, 3 Faculty of Biology and Psychology, Goettingen \\ University, Goettingen, Germany \\ * taoyao@dpz.eu (TY); skrishna@dpz.eu (BSK)
}

\begin{abstract}
We experience a visually stable world despite frequent retinal image displacements induced by eye, head, and body movements. The neural mechanisms underlying this remain unclear. One mechanism that may contribute is transsaccadic remapping, in which the responses of some neurons in various attentional, oculomotor, and visual brain areas appear to anticipate the consequences of saccades. The functional role of transsaccadic remapping is actively debated, and many of its key properties remain unknown. Here, recording from two monkeys trained to make a saccade while directing attention to one of two spatial locations, we show that neurons in the middle temporal area (MT), a key locus in the motion-processing pathway of humans and macaques, show a form of transsaccadic remapping called a memory trace. The memory trace in MT neurons is enhanced by the allocation of top-down spatial attention. Our data provide the first demonstration, to our knowledge, of the influence of top-down attention on the memory trace anywhere in the brain. We find evidence only for a small and transient effect of motion direction on the memory trace (and in only one of two monkeys), arguing against a role for MT in the theoretically critical yet empirically contentious phenomenon of spatiotopic feature-comparison and adaptation transfer across saccades. Our data support the hypothesis that transsaccadic remapping represents the shift of attentional pointers in a retinotopic map, so that relevant locations can be tracked and rapidly processed across saccades. Our results resolve important issues concerning the perisaccadic representation of visual stimuli in the dorsal stream and demonstrate a significant role for top-down attention in modulating this representation.
\end{abstract}

\section{Author Summary}

Humans experience a visually stable world despite the fact that eye, head, and body movements cause frequent shifts of the image on the retina. Humans and monkeys are also able to keep track of visual stimuli across such movements. One mechanism that may contribute to these abilities is "transsaccadic remapping," in which the responses of some neurons 
Competing Interests: The authors have declared that no competing interests exist.

Abbreviations: FEF, frontal eye field; LIP, lateral intraparietal area; MST, medial superior temporal area; MT, middle temporal area; PSTH, peristimulus time histogram; RDP, random dot pattern; RF, receptive field; SC, superior colliculus. in various attentional, oculomotor, and visual brain areas appear to anticipate the consequences of saccades. A current hypothesis proposes that the brain maintains "attentional pointers" to the locations of relevant stimuli and that, via transsaccadic remapping, it rapidly relocates these pointers to compensate for intervening eye movements. Whether stimulus features are also remapped across saccades (along with their location) remains unclear. Here, we show the presence of transsaccadic remapping in a macaque monkey brain area critical for visual motion processing, the middle temporal area (MT). This remapped response is stronger for an attended stimulus. We find only weak evidence for motion-direction information in the remapped response. These results support the attentional pointer hypothesis and demonstrate for the first time, to our knowledge, the impact of top-down attention on transsaccadic remapping in the brain.

\section{Introduction}

Prior research has revealed the potential contribution to visual processing of transsaccadic remapping, in which some neurons in the lateral intraparietal area (LIP), frontal eye field (FEF), superior colliculus (SC), medial superior temporal area (MST), and ventral stream (areas V3a, V3, and V2) respond perisaccadically as long as a visual stimulus could be anticipated in their receptive fields (RFs) after the saccade [1-7]. This "remapped response" is not a simple visual afferent response, because it appears even when the visual stimulus disappears just before the saccade (that would bring the stimulus location into the RF), so that no stimulus ever appears in the neurons' visual RF before or after the saccade. Furthermore, in some neurons, it begins with a latency shorter than the normal visual latency and can even begin before saccade onset, in which case it has been referred to as "predictive remapping" [1]. More commonly, the remapped response occurs postsaccadically, and when this occurs in a situation in which there is no postsaccadic stimulus in the RF because it disappeared before the saccade, the remapped response is referred to as a "memory trace" of the location of the visual stimulus [1].

The functional role of this remapped response is currently being actively debated, and many of its key response properties remain unknown. It has been proposed [8] that transsaccadic remapping represents the predictive, presaccadic shift of attentional pointers on a retinotopic map that keeps track of attended locations across saccades, so that attended locations can be preferentially processed with minimal delay after the saccade. This reduction of delay would be especially helpful when planning rapid sequential saccades and could also help maintain an uninterrupted visual experience across saccades. Others have suggested that this view may be too restrictive and that information about visual features are also remapped across saccades, in addition to location [9-12]. This alternative view thus invokes an additional role for transsaccadic remapping in spatiotopic feature comparison and adaptation transfer across saccades. Resolving these issues requires a better understanding of the properties of the remapped response in different brain areas. Here, we address and answer several open questions regarding the remapped response in the middle temporal area (MT), a key motion processing area, in the rhesus macaque.

MT is an important locus in the processing of visual motion and is strongly interconnected with LIP, FEF, SC, and MST. A previous report from macaque MT showed the absence of predictive remapping [13] in MT neurons; our results are consistent with this. Another recent report reported the presence of a memory trace in MST neurons but failed to find a memory trace in a small sample of MT neurons using a paradigm with a flashed visual stimulus; the 


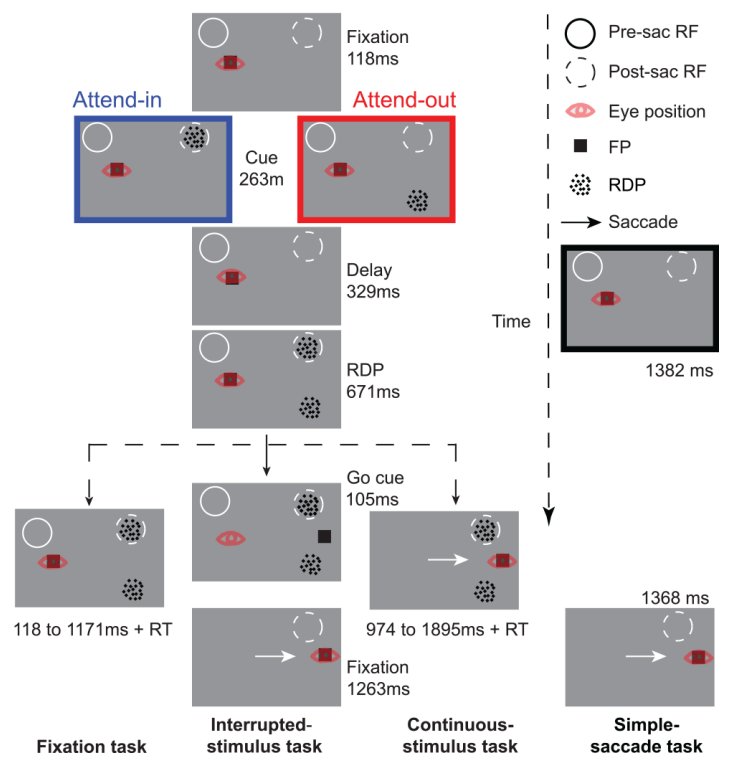

Fig 1. Task design and timing. Two rhesus monkeys were trained to perform a task that involved attending to one of two moving RDPs while also making a visually guided saccade if the fixation point jumped to a new location (continuous-stimulus task and fixation task). On about $44 \%$ of trials, the RDPs disappeared just before the saccade (interrupted-stimulus task). On about $11 \%$ of trials, RDPs were never presented and the monkey only had to make a visually guided saccade (simple-saccade task). Values next to each panel represent the durations of the task phase represented by that panel. For details, see Materials and Methods and $\mathrm{S} 1$ Text.

doi:10.1371/journal.pbio.1002390.g001

authors therefore suggested that the memory trace may be an emergent property that differentiates MST from MT [2]. Here, we report that MT neurons do show a memory trace, using an experimental paradigm that requires the monkey to pay top-down attention to one of two motion stimuli. Furthermore, we show that the memory trace in MT neurons is enhanced by top-down spatial attention. This is the first demonstration, to our knowledge, of the influence of top-down attention on the memory trace in any brain area. Finally, we find evidence only for a weak and transient effect of motion direction on the memory trace in one monkey. Our data are therefore consistent with the hypothesis that transsaccadic remapping represents the shift of attentional pointers in a retinotopic map. Our results further clarify the perisaccadic representation of visual stimuli in the dorsal stream and demonstrate a significant role for topdown attention in modulating this representation.

\section{Results}

We report the responses of $90 \mathrm{MT}$ neurons, 46 from monkey $\mathrm{H}$ and 44 from monkey $\mathrm{E}$. We first considered the responses from 0 to $500 \mathrm{~ms}$ after saccade offset (see Methods) in the continuous-stimulus task (Fig 1), in which the monkeys made a saccade that brought either the (previously cued) target random dot pattern (RDP) or the distractor RDP into their RF, and no stimulus was present in the RF before the saccade. As previously reported (e.g., [14-16]), neurons showed a clear postsaccadic enhancement for attended (solid blue curves, Fig 2A and 2B) versus unattended (dotted red curves, Fig 2A and 2B) RDPs moving in the preferred direction in their RF. Compared to the distractor RDP, the response to the (cued) target RDP moving in 


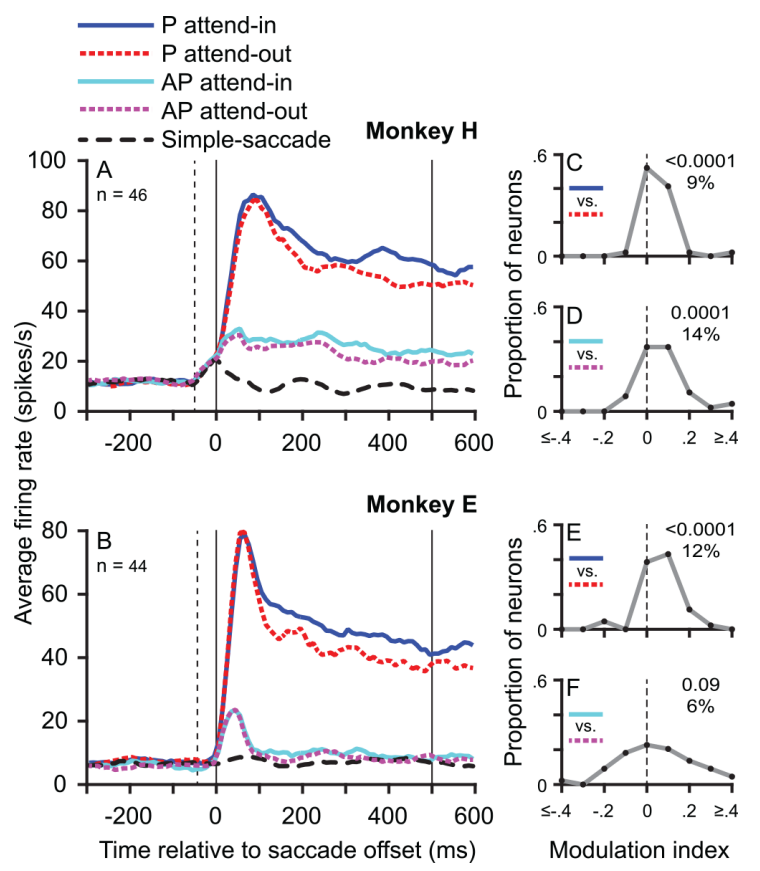

Fig 2. Attentional enhancement of MT neuronal responses to moving RDPs. $(A, B)$ Population average peristimulus time histograms (PSTHs) for monkey $\mathrm{H}(\mathrm{A})$ and monkey $E(B)$ in response to attended (target) and unattended (distractor) RDPs moving either in the preferred $(P)$ or antipreferred (AP) direction (see legend at top left). The PSTH for the simple-saccade task is also shown in black as a reference. Solid vertical lines demarcate the time window used for computing the modulation indices in C-F. The dotted vertical line indicates the mean time of saccade onset. $(\mathrm{C}, \mathrm{E})$ Frequency polygons of the distribution of modulation indices in monkey $\mathrm{H}(\mathrm{C})$ and monkey $\mathrm{E}(\mathrm{E})$ when the preferred direction RDP is in the RF after the saccade show a clear predominance of values greater than zero, i.e., an enhanced response in the attend-in conditions. The $p$-value from the signed-rank test of the modulation indices and the median modulation index (converted to a percentage and rounded) are shown on the top right of each panel. The final point in the frequency polygons sums all data values at or beyond the extreme value. (D,F) Same as C,E but for the antipreferred direction RDP. Data in Supporting Information (S1 Data).

doi:10.1371/journal.pbio.1002390.g002

the preferred direction in a time window of 0 to $500 \mathrm{~ms}$ following saccade offset was greater by a median value of $8.9 \%$ in monkey $\mathrm{H}$ (Fig $2 \mathrm{C}, p<0.0001$ ) and $12.4 \%$ in monkey $\mathrm{E}$ (Fig $2 \mathrm{E}$, $p<0.0001)$. The attentional modulation of an antipreferred direction target was significant in monkey H (Fig 2D, $13.7 \%, p=0.0001$ ) but not in monkey E (Fig $2 \mathrm{~F}, 5.8 \%, p=0.0909$ ). Consistent with a prior report [13], we observed no predictive remapping, i.e., no presaccadic increase in activity in MT neuronal responses (Figs 2A, 2B and S1).

\section{MT Neurons Show a Memory Trace That Is Enhanced by Top-Down Attention}

In contrast to the continuous-stimulus task above, the RDPs in the interrupted-stimulus task (Fig 1) disappear before saccade onset. Thus, a neuronal response after the saccade would represent a memory trace and not a sensory response, since there is no stimulus in the postsaccadic RF (or in the presaccadic RF). To determine the presence of a memory trace, we considered the responses in the interrupted-stimulus task when the target RDP (irrespective of motion direction) was at the postsaccadic RF location before the saccade (attend-in interrupted- 


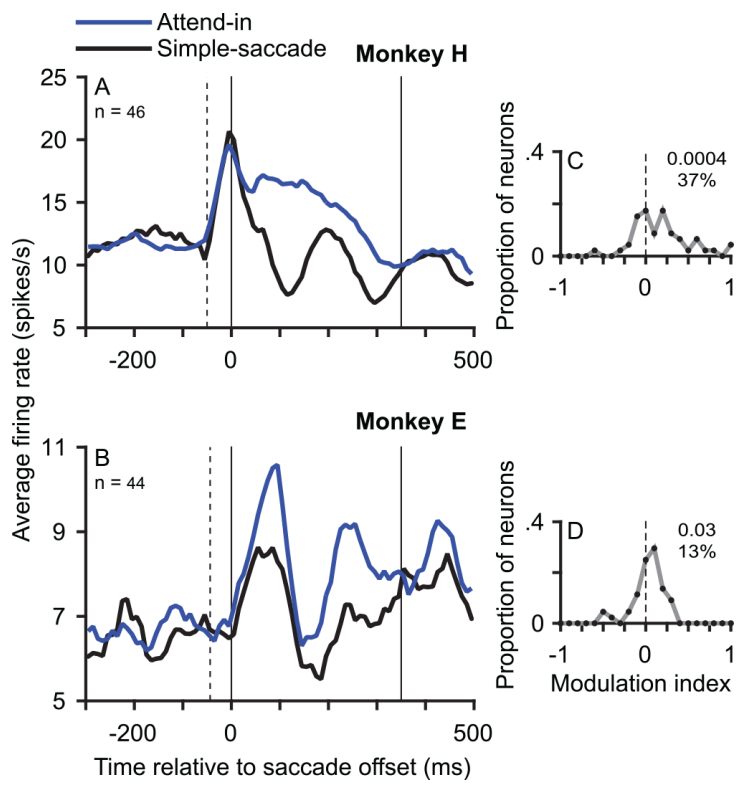

Fig 3. MT neurons show a memory trace. The memory trace is an enhanced postsaccadic response (compared to the simple-saccade) when a moving target RDP was presented (only before the saccade) at their postsaccadic RF location. We pooled the responses to the two RDP directions because we did not find an influence of RDP motion direction on the memory trace (Fig 5). (A,B) Population average PSTHs for monkey $\mathrm{H}(\mathrm{A})$ and monkey $\mathrm{E}(\mathrm{B})$ in the attend-in condition of the interrupted-stimulus task (blue) compared to the simple-saccade task (black). The $y$-axes in $A$ and $B$ have different ranges. (C,D) Frequency polygons of the distribution of modulation indices (for the response from 0 to $350 \mathrm{~ms}$ after saccade offset) comparing these two conditions in monkey $\mathrm{H}(\mathrm{C})$ and monkey $\mathrm{E}(\mathrm{D})$ show a clear predominance of values greater than zero, i.e., an enhanced response in the attend-in condition. Conventions as in Fig 2. Data in Supporting Information (S2 Data).

doi:10.1371/journal.pbio.1002390.g003

stimulus condition). We compared this response from 0 to $350 \mathrm{~ms}$ after saccade offset (see Methods) to the response in the same time window in the simple-saccade task where the monkey only made a saccade with no RDP ever appearing on the screen (Fig 3). We found a strong enhancement of responses in the attend-in condition of the interrupted-stimulus task compared to the simple-saccade task (Fig $3 \mathrm{~A}$ and $3 \mathrm{~B}$, blue curve versus black curve), and we interpret this enhancement as a memory trace of the visual stimulus presented before the saccade. The median enhancement of the response following saccade offset in the attend-in interrupted-stimulus condition was $37.4 \%(p=0.0004)$ in monkey $\mathrm{H}$ and $13.1 \%(p=0.0308)$ in monkey E.

In order to examine the effect of attention on the memory trace, we then compared the memory trace for the target (in the attend-in interrupted-stimulus condition) to that for the distractor RDP (in the attend-out interrupted-stimulus condition): in both cases, the RDP was in the postsaccadic RF before the saccade but not after it. The memory trace for the target was clearly greater than that for the distractor RDP (Fig 4A and 4B, blue curve versus red curve). The median enhancement of the memory trace for the target relative to that for the distractor was $25.4 \%(p<0.0001$, Fig $4 \mathrm{C})$ in monkey H and $14.1 \%(p=0.0022$, Fig $4 \mathrm{E})$ in monkey E. The memory trace for the distractor, on the other hand, was either weak or absent. Based on the modulation indices, the memory trace for the distractor was not significantly different from when there was no stimulus in the simple-saccade condition (Fig 4A and 4B, red curve 


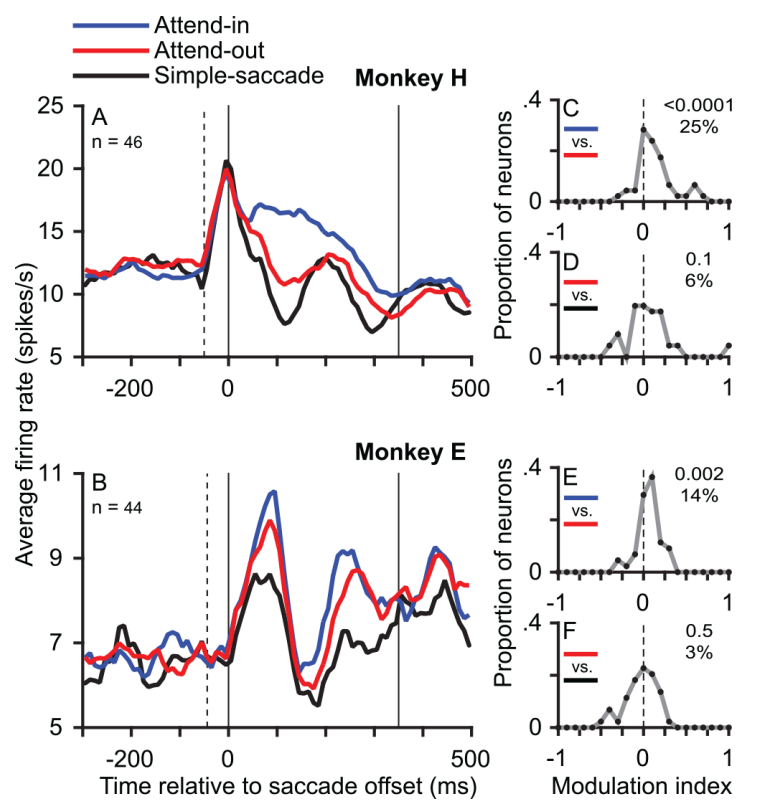

Fig 4. The memory trace is sensitive to top-down attention. The postsaccadic response is larger when a target RDP (as compared to a distractor RDP) was in the postsaccadic RF location before the saccade. (A,B) Population average PSTHs for monkey $\mathrm{H}(\mathrm{A})$ and monkey $\mathrm{E}(\mathrm{B})$ in the attend-in condition (blue) and attendout condition (red) of the interrupted-stimulus task, pooled across motion directions as well as the simplesaccade task (black). The $y$-axes in A and B have different ranges. (C,E) Frequency polygons of the distribution of modulation indices comparing the attend-in and attend-out conditions in monkey $\mathrm{H}(\mathrm{C})$ and monkey $E(E)$ show a clear predominance of values greater than zero, i.e., an enhanced response in the attend-in condition. (D,F) Frequency polygons of the distribution of modulation indices comparing the attendout condition of the interrupted-stimulus task and the simple-saccade task in monkey $H(D)$ and monkey $E(F)$ show no significant difference in the responses. Conventions as in Figs 2 and 3. Data in Supporting Information (S3 Data).

doi:10.1371/journal.pbio.1002390.g004

compared to black curve): the response in the attend-out condition was larger by $6.4 \%$ $(p=0.1417$, Fig $4 \mathrm{D})$ in monkey $\mathrm{H}$ and by $2.7 \%(p=0.5222$, Fig $4 \mathrm{~F})$ in monkey E. However, this lack of significance appears to contrast with the effect that is visible in the average population PSTHs (red versus black curves in Fig 4A and 4B). This is because the separation between the average population PSTHs reflects the difference between the mean firing rates in the two conditions, while the median modulation index is a measure based on the ratio of firing rates. Performing a paired $t$ test between the firing rates in the attend-out and simple-saccade condition does reveal a significant enhancement in the attend-out condition (Monkey H: mean difference $=1.3$ spikes per second, $p=0.0450$; Monkey E: mean difference $=0.8$ spikes per second, $p=0.0270)$.

\section{The MT Memory Trace Only Shows a Transient Effect of Motion Direction (In One Monkey)}

We examined whether the attention-sensitive memory trace in MT also contains information about the motion direction of the stimulus that elicited the memory trace, whether it be the target or the distractor RDP. We calculated the responses for trials in which the preferred or antipreferred direction RDP (as identified from the continuous-stimulus task) was in the 


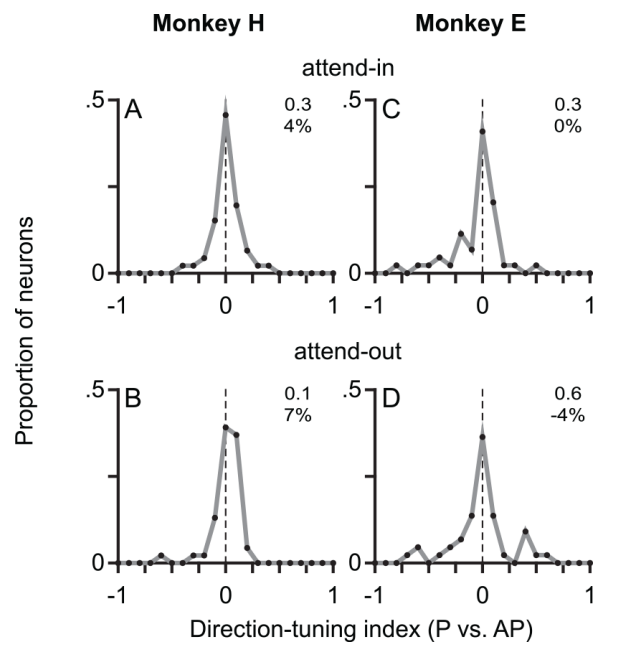

Fig 5. The memory trace (from 0 to $350 \mathrm{~ms}$ ) is not sensitive to motion direction. The postsaccadic response is not significantly different when the preferred $(P)$ or antipreferred $(A P)$ direction RDP was in the postsaccadic RF location before the saccade. The four panels show frequency polygons of the distribution of direction-tuning indices (see Methods) for the attend-in $(A, C)$ and attend-out $(B, D)$ conditions of the interrupted-stimulus task (for the response from 0 to $350 \mathrm{~ms}$ after saccade offset). Results for monkey $\mathrm{H}$ in the left column $(A, B)$ and for monkey $E$ in the right column $(C, D)$. None of the distributions show a statistically significant deviation from zero. Preferred and antipreferred directions were defined based on the response in the continuous-stimulus task. Other conventions as in Figs 2-4. Data in Supporting Information (S4 Data).

doi:10.1371/journal.pbio.1002390.g005

postsaccadic RF. We did not find any significant effect of motion direction when we compared the responses in either the attend-in condition (with the target RDP in the postsaccadic RF location) or the attend-out condition (with the distractor RDP in the postsaccadic RF location). None of the response differences (Fig 5A-5D) were statistically significant (all $p$ values $>0.1248)$. Additionally, since the preferred and antipreferred directions defined on the basis of the responses in the continuous-stimulus task may not predict the memory trace in the interrupted-stimulus task, we used a two-fold approach. We first computed the response after saccade offset on even-numbered trials and designated the motion direction that elicited the larger response as the preferred direction. We then used odd-numbered trials to perform the same analysis of the effects of motion direction on the memory trace. Once again, none of the response differences were statistically significant (all $p$-values $>0.1292$ ).

Since it is possible that motion-direction selectivity may be present in the memory trace at shorter time scales, we also examined the motion-direction selectivity of the memory trace over shorter time periods (Fig 6). There was no evidence for motion-direction selectivity in the memory trace for monkey $\mathrm{H}$ in either the attend-in (Fig 6A and 6E) or the attend-out (Fig 6B and $6 \mathrm{~F}$ ) conditions, as evidenced by the fact that the $95 \%$ confidence bands (Fig $6 \mathrm{E}$ and $6 \mathrm{~F}$ ) included zero throughout the time course and none of the nonoverlapping statistical comparisons (Bonferroni-corrected for multiple comparisons) were statistically significant. The results from monkey E were similar, except that there was a transient effect of motion direction on the memory trace (Fig 6C and 6G) in the attend-in condition, where the memory trace for the nonpreferred direction was larger in the time window from 50 to $100 \mathrm{~ms}$ after saccade offset ( $p=0.0031$ for the direction-tuning index and 0.0024 for the difference in firing rates). Examining the response in this time window in each individual neuron (using a rank-sum test comparing the responses to the preferred and nonpreferred directions) did not yield significance 


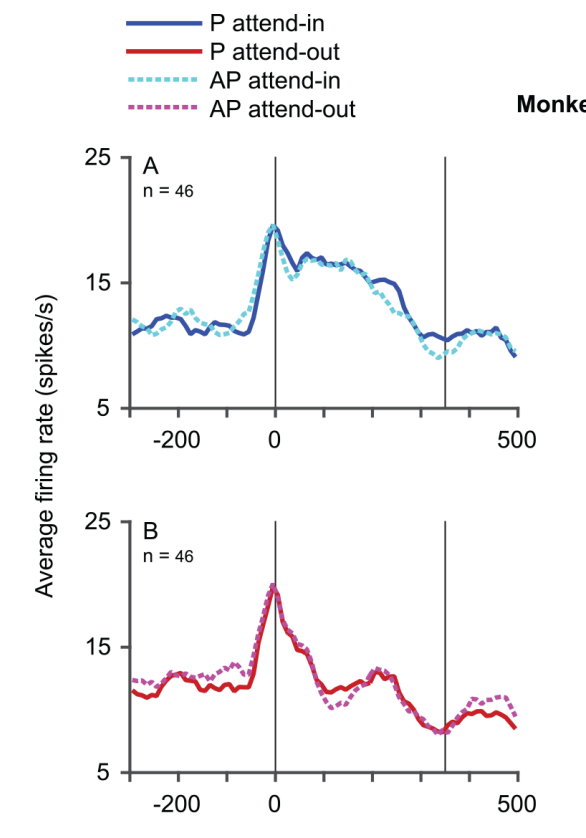

onkey $\mathrm{H}$
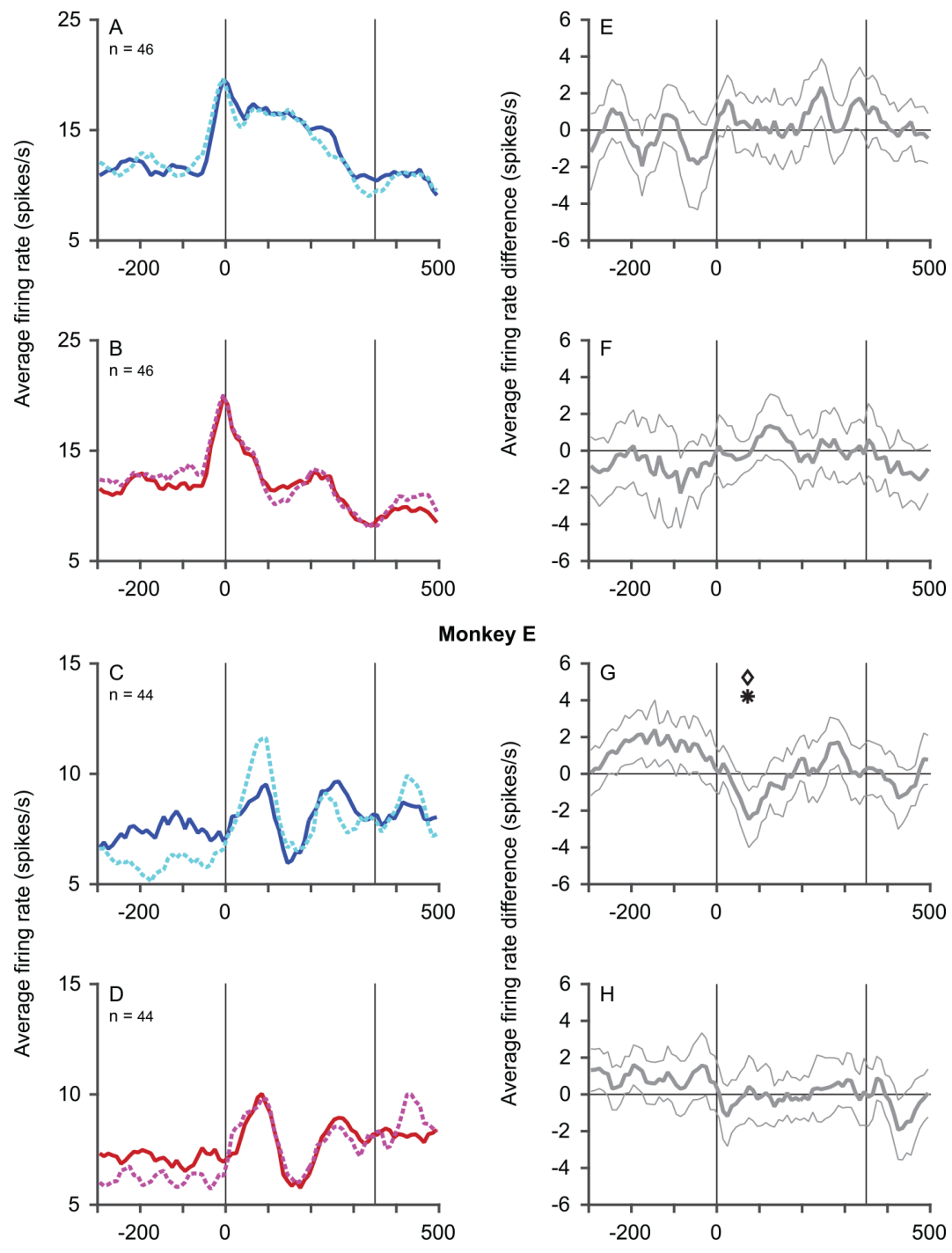

Monkey E
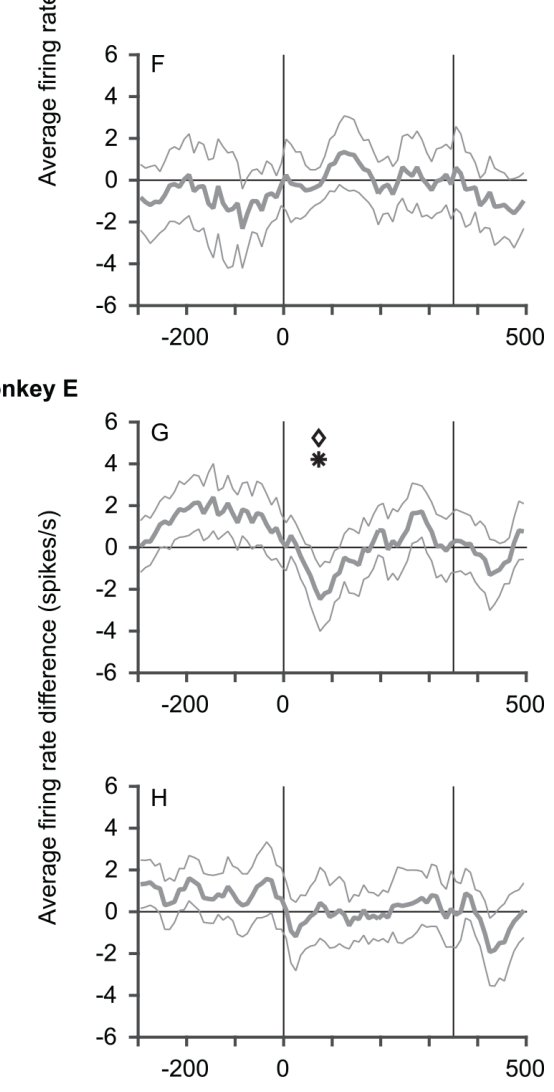

Time relative to saccade offset $(\mathrm{ms})$

Fig 6. Evidence only for transient feature-related information in the memory trace in one monkey. In the left column, population average PSTHs for the preferred direction (blue trace in A,C and red trace in B,D) and nonpreferred direction (cyan trace in $A, C$ and magenta trace in $B, D$ ) for the attend-in $(A, C)$ and attend-out $(B, D)$ conditions of the interrupted-stimulus task are plotted. Preferred and nonpreferred directions were determined from the responses in the continuous-stimulus task. In the right column, the mean difference (averaged across neurons) between the PSTH for the preferred and nonpreferred direction is plotted (along with the $95 \%$ confidence bands) for the attend-in $(E, G)$ and attend-out $(F, H)$ conditions. All PSTHs calculated using overlapping $50 \mathrm{~ms}$ bins, stepping every $10 \mathrm{~ms}$. Data from monkey $\mathrm{H}(\mathrm{A}-\mathrm{B}, \mathrm{E}-\mathrm{F})$ and monkey $\mathrm{E}(\mathrm{C}-\mathrm{D}$, G-H). Statistical significance was calculated (using signed-rank tests Bonferroni-corrected for 16 comparisons) using the difference between firing rates (diamond symbols) as well as using the modulation 
index (asterisk symbol) in nonoverlapping $50 \mathrm{~ms}$ windows over the entire analysis period ( 16 comparisons over $800 \mathrm{~ms}$ from -300 to $500 \mathrm{~ms}$ relative to saccade offset). Only one time bin (G) showed significance. Other conventions as in Fig 2. Data in Supporting Information (S5 Data).

doi:10.1371/journal.pbio.1002390.g006

for any neuron. Also, using a more liberal false-discovery rate correction for multiple comparisons did not change the result for the post-saccadic time bins, but indicated a significant effect of motion direction before the saccade (as suggested by the PSTHs in Fig 6C, with the responses in the blue trace being slightly larger than the cyan trace). These presaccadic effects could reflect weak stimulus-driven effects from outside the RF [17] and/or the effects of feature-based attention [18].

In both monkeys, a transient increase in activity that starts either before or immediately after saccade offset is visible in the average population PSTHs from all three tasks: the simplesaccade task, the interrupted-stimulus task (Fig $4 \mathrm{~A}$ and $4 \mathrm{~B}$ ), and the continuous-stimulus task (Fig 2A and 2B). For the simple-saccade task, similar responses have been reported before, with substantial variability in individual neurons ([19,20]; also see Discussion). The apparent difference in time course in this response between the two monkeys may represent differences in the sampled population of neurons, since a subset of neurons (with more eccentric RFs) from monkey E shows a time course quite similar to that in monkey $\mathrm{H}$. The genesis and properties of this response difference, though not fully understood, are beyond the focus of this paper, since our experiment was not designed to study it.

\section{Discussion}

We report that MT neurons show a memory trace: they respond more strongly after a saccade when a stimulus is present only before the saccade in their postsaccadic RF location. Furthermore, we show that the memory trace is stronger for an attended stimulus and does not contain information about motion direction. A memory trace has been shown previously in areas like LIP, FEF, SC, and MST [1-3,5,6], with which MT is strongly anatomically connected [21-23]. Another recent study by Inaba and Kawano [2] reported that a sample of 46 MT neurons did not carry information about the location of a recently disappeared stimulus in their postsaccadic response; they only found such information in the responses of MST neurons and concluded that the memory trace was an emergent property of MST neurons. Our task differs from theirs because it required the monkey to pay (top-down) attention to one of two stimuli, while their task only required the monkey to make a simple, visually guided saccade and the stimulus used to probe the memory trace was task-irrelevant. The difference between our results might therefore be partially explained by our result that MT neurons show a clear memory trace for attended stimuli, with the memory trace for unattended stimuli being weak or absent. However, other aspects might also contribute. First, the two studies probably sampled different kinds of neurons: the study by Inaba and Kawano excluded neurons that showed a response in the simple-saccade task, while the neurons in our sample show a transient response in the simple-saccade task (Fig 3). Such a transient response has been reported before from area MST [19] as well as area MT, even following saccades made in the dark (Ibbotson, M.R., personal communication, even though an earlier report from Ibbotson and colleagues [24] reported the absence of such a response in a small sample of 17 MT neurons). Further supporting the possibility that different neurons were sampled, the study by Inaba and Kawano reported that MT neurons showed a substantially longer latency (relative to saccade offset) to stimuli brought into their RFs by a saccade compared to stimuli flashed in their RF. This differs from the conclusion reached by another recent study [13] in which no difference was found between the two latencies. Though we do not present an analysis of response latency here, the 
average population PSTHs (Fig 2A and 2B) suggest that the average latency is not longer than that expected from previous reports on MT neurons (between 30 and $40 \mathrm{~ms}[2,13]$ ). Second, the polarity of the stimuli used was also different: the study by Inaba and Kawano used a white stimulus on a dark background, while we used a dark stimulus on a white background, and such polarity differences are known to have strong effects on the responses of V1 neurons [25]. Finally, the study by Inaba and Kawano relied on a receiver operating characteristic analysis performed within a sliding $10 \mathrm{~ms}$ window to report the absence of spatial tuning in the postsaccadic response 0 to $100 \mathrm{~ms}$ after saccade offset. Based on our results, it is possible that the 10 ms window may be too narrow and the $100 \mathrm{~ms}$ window too short to reliably detect tuning.

We show that the memory trace in MT neurons is larger for an attended stimulus; this is the first demonstration of the influence of top-down attention on the memory trace in any brain area. The task required the monkey to attend to the stimulus location throughout the trial. This stimulus location lay outside the recorded neuron's RF before the saccade and inside it only after the saccade. Therefore, assuming a single locus of attention, the monkey would have to shift attention from its presaccadic location on a retinotopic map (outside the RF) to its postsaccadic location (inside the RF) right around the saccade. It is also possible that two loci of attention simultaneously exist and that, around the saccade, attention is allocated simultaneously to both task-relevant locations (the presaccadic and postsaccadic stimulus locations on the retinotopic map). Previous psychophysical data from humans indicate that attentional effects are visible at the postsaccadic retinotopic location of a task-relevant stimulus shortly before saccade onset [26-28]. In our task, the neural data from MT indicate that attentional effects emerge in MT soon after saccade offset, but not before that (Figs 2 and S2). Based on our results, we suggest that the memory trace can be explained as the postsaccadic enhancing effect of a perisaccadic allocation of attention to the RF location (on a retinotopic map), where the monkey expects the target to be. Since there is no longer a stimulus in the neuron's RF, attention acts on the baseline, stimulus-independent activity to produce the memory trace. Psychophysically, attentional effects may have been visible in our task either before the saccade (though MT only shows postsaccadic attentional effects) or after the saccade (around the same time as the emergence of attentional effects in MT); since our task design did not allow us to measure the dynamics of attention psychophysically, we cannot distinguish between these two possibilities. Our interpretation of the memory trace as reflecting a top-down attentional effect in our task is consistent with previous findings showing stronger remapped responses for salient or task-relevant stimuli: LIP neurons show stronger levels of anticipatory (predictive) remapping to the appearance of a visual search target [29] or saccade target [30] in their RF after a saccade, compared to the appearance of a distractor. Similarly, stimuli with greater bottom-up saliency have been shown to elicit stronger remapped responses in LIP [30] and FEF [31]. When using a measure based on the difference of spike rates, our data indicate a weak memory trace for the unattended stimulus. However, this effect is not present when using a measure based on the ratio of spike rates. More data with a greater number of stimuli are needed before reaching more general conclusions about the extent to which unattended stimuli are also transsaccadically remapped.

The phenomenology of presaccadic remapping of visual RFs measured using flashed stimuli is currently controversial. The classical position in the literature is that neurons that show predictive remapping in LIP [1], FEF [4], and SC [6] are anticipating the appearance of a stimulus in their postsaccadic RF (as if they are shifting their RFs preemptively to their future postsaccadic locations). In contrast, it has recently been proposed [32,33], based on recordings from FEF, that such remapping actually represents a transient shift of visual RFs toward the saccade target and that this transient shift is correlated with the attentional shift to the saccade target before the saccade $[34,35]$. While these two views await reconciliation, we emphasize that our 
experimental design and interpretation of the memory trace in MT is not dependent on either of these competing accounts of the phenomenology of predictive visual RF shifts measured using flashed stimuli. Our design and interpretation instead depend only on the fairly large body of evidence supporting spatially accurate remapping: psychophysical evidence from double-step experiments [36], free-viewing visual search [37], and transsaccadic attentional measurements [15,27] all show that the locations of salient stimuli and future saccade targets are remapped rapidly and accurately across saccades. Similarly, LIP $[29,30]$ and FEF [38] neurons anticipatorily signal the presence of a target in their RFs. SC, LIP, and FEF neurons signal the location of the impending second saccade within their RFs in a double-step task [39-42]. SC neurons also rapidly compensate for midsaccade deviations in eye position introduced by electrical stimulation in the SC during a saccade $[43,44]$. The relationship of this spatially accurate remapping mechanism to the contentious spatial properties of predictive visual RF shifts is unclear at present.

The presence of feature-related information in the remapped response has become a critical test that distinguishes between two alternative views of the functional role of transsaccadic remapping that are being actively debated $[8,9,11,32,45]$. Absence of featural information in the remapped response would support the proposal [8] that transsaccadic remapping represents the predictive, presaccadic shift of attentional pointers on a retinotopic map that keeps track of attended locations across saccades. On the other hand, the presence of featural information in the remapped response would support the proposal that transsaccadic remapping plays an additional role in spatiotopic feature comparison and adaptation transfer across saccades [9-12], though the data on adaptation transfer have not been universally replicated (summarized in [46]). Our data clearly indicate that any motion-direction information present in the remapped response is weak: in our data, it was only present transiently in one monkey. This, combined with the greater memory trace elicited by the attended stimulus, indicates that the memory trace in MT neurons predominantly represents the effects of a shift of attentional pointers. We note here that evidence for featural information in the remapped response has been presented recently from LIP [47], and neurons that signal transsaccadic changes in stimulus location and/or color in their postsaccadic reafferent response have been found in FEF (though its relationship to remapping is unclear [12]). However, these areas only show coarse tuning to stimulus features, and our data provide the first set of evidence (against a featuretuned remapped response) from a sensory area with neurons that are more finely tuned to stimulus features. The weak effect found in our data may either reflect a feature-selective input to the remapped response or simply the effects of response adaptation given the slightly higher response in the presaccadic period when the monkey was attending to the preferred direction outside the RF. These presaccadic effects could themselves reflect weak stimulus-driven effects from outside the RF [17] and/or the effects of feature-based attention [18].

MT neurons do not show anticipatory remapping [13]; we hypothesize that the anticipatory remapping seen in attentional and oculomotor control areas like LIP, FEF, and SC is part of the process that switches the attentional pointer, and though this process starts before the saccade in these areas, its effects in MT only manifest after the saccade (at a point of time when the pointer is again at the task-relevant location). The anticipatory nature of the remapping seen in LIP, FEF, and SC may confer an evolutionary advantage by ensuring that attention is allocated to the correct retinotopic location soon after the saccade. A recent psychophysical study [28], using a motion task similar to ours, observed a decrement in performance at attended locations before a saccade and suggested that this resulted from the known reallocation of attention to the saccade target [34,48-50] and/or the remapped location [27]. Our data from MT do not indicate any evidence for a presaccadic shift of attention to the remapped location. Recordings from areas upstream of MT along the motion-process pathway combined with psychophysical measurements of perisaccadic attentional dynamics are needed before the neural basis of these 
processes can be understood. Our current results, when combined, resolve important issues concerning the perisaccadic representation of visual stimuli in the dorsal stream and demonstrate a significant role for top-down attention in modulating this representation.

\section{Materials and Methods}

\section{Statement on Animal Research within This Study}

All animal work was conducted according to the relevant national and international guidelines. All animal procedures have been approved by the responsible regional government office (Niedersächsisches Landesamt für Verbraucherschutz und Lebensmittelsicherheit [LAVES]) under the permit numbers 33.14.42502-04-064/07 and 3392 42502-04-13/1100.

The animals were group-housed with other macaque monkeys in facilities of the German Primate Center in Goettingen, Germany in accordance with all applicable German and European regulations. The facility provides the animals with an enriched environment (including a multitude of toys and wooden structures) exceeding the size requirements of the relevant European regulations.

All invasive procedures were done under appropriate anesthesia and with appropriate analgesics. The German Primate Center has several veterinarians on staff that regularly monitor and examine the animals and consult on any procedures.

During the study, the animals had unrestricted access to food and fluid, except on the days when data were collected or the animal was trained on the behavioral paradigm. On these days, the animals were allowed unlimited access to fluid through their performance in the behavioral paradigm. Here, the animals received fluid rewards for every correctly performed trial. Throughout the study, the animals' psychological and veterinary wellbeing was monitored by the veterinarians, the animal facility staff, and the lab's scientists, all specialized on working with nonhuman primates.

Both of the animals used in the study are currently in other studies in our laboratory.

\section{General}

We trained two male rhesus monkeys (Macaca mulatta), monkey $\mathrm{H}$ and monkey E, to perform a demanding visuospatial-attention task along with a saccade. Each monkey was implanted with a titanium head holder and a recording chamber located above the parietal cortex (based on a MRI scan) to allow MT recordings. All surgical procedures were approved by the district government of Lower Saxony, Germany, and were conducted under general anesthesia using standard techniques. The experiments were performed in a dimly lit room, and the monkey viewed a CRT monitor $(76 \mathrm{~Hz})$ while sitting in a custom-made primate chair during the experiment (see S1 Text for detailed Methods). All aspects of the experiment were controlled by custom software running on an Apple Macintosh computer. The eye position was monitored by an EyeLink 1000 (SR Research, Canada) system at 1,000 Hz. Neuronal activity was recorded extracellularly with a 5-channel micro drive system (Mini Matrix, Thomas Recording, Giessen, Germany) and processed using the Plexon data acquisition system (Plexon Inc., Dallas, TX, United States). Only data from well-isolated neurons are reported here. MT was identified by referencing the recordings to the structural MRI and by the physiological properties of the recorded neurons.

\section{Behavioral Tasks and Stimuli}

Once a neuron was isolated, we mapped its RF location and determined the neurons' preferred direction and speed while the monkeys performed a fixation task. We then switched to the main experiment (Fig 1), in which each trial was composed of one of four tasks (three 
experimental tasks and one control task, chosen in a pseudo-randomly interleaved manner). In all four tasks, the monkeys initiated the trial by holding a metal bar and foveating a black fixation point. In the control task (the "simple-saccade" task, $11.1 \%$ of trials), the monkeys had to maintain fixation until a saccade target (identical to the fixation point) appeared and the fixation point disappeared (see S1 Text for details). The monkeys had to make a saccade to the saccade target and maintain fixation there until the end of the trial to obtain a reward. In the three experimental tasks, in addition to potentially making a saccade as in the simple-saccade task, the monkeys had to attend to one of two moving RDPs (both moving in the same direction, which was either the neuron's preferred or antipreferred direction) and respond to a brief (132 ms) direction change in this target by releasing the bar, but ignore similar changes in the other RDP (the distractor). The target stimulus was cued by a stationary RDP that appeared at its location for $263 \mathrm{~ms}$. The target and distractor stimuli were always equidistant from the fixation point and saccade target and were always mirrored with respect to the saccade target (Fig 1), so that for horizontal saccades, they appeared in the upper and lower hemifield (and the left or right hemifield, if the $\mathrm{RF}$ was located in the left [monkey $\mathrm{H}$ ] hemifield or right [monkey $\mathrm{E}$ ], respectively). The cue appeared equally often in the postsaccadic RF (attend-in condition) or opposite to it (attend-out condition). In addition, during the trial, if the fixation point jumped to a new location (as in the simple-saccade task), the monkeys had to refixate the fixation point while continuing to attend to the cued target. In the first of the three experimental tasks (the "continuous-stimulus task," $22.2 \%$ of trials), the fixation point jumped to its new location $671 \mathrm{~ms}$ after RDP onset. The direction change in the target RDP could occur between 974 and 1,895 ms after the fixation point jumped. The second experimental task (the "interrupted-stimulus task," $44.4 \%$ of trials) was similar to the continuous-stimulus task, but the target and distractor RDPs disappeared $105 \mathrm{~ms}$ after the fixation point jumped and, therefore, no stimulus ever appeared in the neurons' RF after the saccade (or before the saccade). The monkeys had to simply make a saccade to the new fixation point location and maintain fixation until the end of the trial to obtain a reward; the few trials with saccades that started before the disappearance of the stimulus were discarded. The third experimental task (the "fixation task," $22.2 \%$ of trials) was also similar to the continuous-stimulus task except that the fixation point never jumped, and the direction change in the target RDP occurred 789 to $1,842 \mathrm{~ms}$ after RDP onset. This task was included to make sure the monkeys paid attention to the target even during the time when they made a saccade in the other two experimental tasks, and was not analyzed further for this study. Distractor changes occurred on about $37.5 \%$ of trials (in the continuous-stimulus and fixation tasks) and never more than once on each trial. The timing of distractor changes overlapped that of target changes, with the additional requirement that any distractor change occurred at least $500 \mathrm{~ms}$ before the target change on each trial. This separation ensured that the monkeys' rare responses to the distractor change could be easily identified and distinguished from their responses to the target change. In all the tasks, the background was always grey, and the fixation point and RDPs, including the stationary cue, were black. Our use of black stimuli addresses concerns regarding the persistence of white visual stimuli on black backgrounds after their stipulated disappearance from a CRT monitor.

\section{Data Analysis}

We detected saccades using a velocity threshold criterion that was validated by visual inspection. We included data from all neurons that showed a significantly greater postsaccadic response to at least one of the two directions in the continuous-stimulus task (compared to the simple saccade task in which there is no stimulus in the RF, i.e., they were visually responsive to the RDP) as well as a significant difference between the responses to the two RDP directions in the continuous-stimulus task (i.e., they showed direction tuning). Additionally, we excluded 
neurons in which the onset of the RDP at the (future) postsaccadic RF location elicited a statistically significant response from the neuron. Only correctly completed trials were analyzed. PSTHs (Figs 2-4) were calculated using partially overlapping bins (50 ms width, stepped every $10 \mathrm{~ms}$ ). For the interrupted-stimulus task (Figs 3-5), we used a time window from 0 to $350 \mathrm{~ms}$ after saccade offset, as a compromise duration that was long enough to make statistically meaningful statements about the effects we observed, and yet not so long that the monkeys would have ample time to withdraw attention from the attended spatial location after realizing that the attended stimulus had disappeared. In addition, $350 \mathrm{~ms}$ is roughly equal to a typical intersaccadic interval. For the continuous-stimulus task (Fig 2), our goal was to merely confirm that we found the attentional effects expected from MT in our dataset. For a precise estimate, we chose a time window of 0 to $500 \mathrm{~ms}$. This choice is not critical, and using a time window of 0 to $350 \mathrm{~ms}$ would not affect our conclusions (though it would provide a less precise estimate). The modulation index was defined as the difference in the firing rates for the two conditions divided by their sum. A direction-tuning index was similarly defined as the difference in firing rates for preferred and antipreferred directions divided by their sum (Fig 5). We report medians and use $p$-values from Wilcoxon signed-rank tests throughout.

\section{Supporting Information}

S1 Fig. The memory trace does not start earlier than the sensory response. The memory trace, plotted as the difference between the response in the attend-in condition of the interrupted-stimulus task and the response in the simple-stimulus task (mean difference across neurons and SEM - red trace), arises at the same time or later than the sensory response, plotted as the difference between the response in the continuous-stimulus task with the preferred direction and the response in the simple-stimulus task (mean difference across neurons and SEMblue trace). The contribution of predictive remapping to the timing of the steep rise of the sensory response toward its peak would be minimal, and the memory trace does not appear to lead the sensory response anywhere along this steep rise. In order to facilitate comparison, both traces were normalized by subtracting the mean value of the trace from -300 to $0 \mathrm{~ms}$ and then dividing by the maximum value. Data for monkey H (A) and monkey E (B). Other conventions as in Fig 2. Data in Supporting Information (S6 Data). (PDF)

S2 Fig. Attentional effects in the continuous task manifest at or after saccade offset. The attentional effect in the continuous-stimulus task for the preferred direction, plotted as the difference between the responses in the attend-in (Fig 2, blue curve) and attend-out (Fig 2, red curve) conditions (mean and $95 \%$ confidence bands), rises above zero only after saccade offset. Data for monkey $\mathrm{H}$ (A) and monkey E (B). Other conventions as in Fig 2. Data in Supporting Information (S7 Data). (PDF)

S1 Data. The data presented in Fig 2 are tabulated in separate text files contained in the zipped folder.

(ZIP)

S2 Data. The data presented in Fig 3 are tabulated in separate text files contained in the zipped folder.

(ZIP)

S3 Data. The data presented in Fig 4 are tabulated in separate text files contained in the zipped folder.

(ZIP) 
S4 Data. The data presented in Fig 5 (and in the associated portion of the Results section) are tabulated in separate text files contained in the zipped folder.

(ZIP)

S5 Data. The data presented in Fig 6 are tabulated in separate text files contained in the zipped folder.

(ZIP)

S6 Data. The data presented in S1 Fig are tabulated in separate text files contained in the zipped folder.

(ZIP)

S7 Data. The data presented in S2 Fig are tabulated in separate text files contained in the zipped folder.

(ZIP)

S1 Text. Detailed Materials and Methods.

(PDF)

\section{Author Contributions}

Conceived and designed the experiments: TY BSK. Performed the experiments: TY BSK. Analyzed the data: TY BSK. Contributed reagents/materials/analysis tools: TY ST BSK. Wrote the paper: TY ST BSK.

\section{References}

1. Duhamel JR, Colby CL, Goldberg ME. The updating of the representation of visual space in parietal cortex by intended eye movements. Science. 1992; 255(5040):90-2. PMID: 1553535

2. Inaba N, Kawano K. Neurons in cortical area MST remap the memory trace of visual motion across saccadic eye movements. Proceedings of the National Academy of Sciences of the United States of America. 2014; 111(21):7825-30. doi: 10.1073/pnas.1401370111 PMID: 24821778

3. Nakamura K, Colby CL. Updating of the visual representation in monkey striate and extrastriate cortex during saccades. Proceedings of the National Academy of Sciences of the United States of America. 2002; 99(6):4026-31. PMID: 11904446

4. Umeno MM, Goldberg ME. Spatial processing in the monkey frontal eye field. I. Predictive visual responses. Journal of neurophysiology. 1997; 78(3):1373-83. PMID: 9310428

5. Umeno MM, Goldberg ME. Spatial processing in the monkey frontal eye field. II. Memory responses. Journal of neurophysiology. 2001; 86(5):2344-52. PMID: 11698524

6. Walker MF, Fitzgibbon EJ, Goldberg ME. Neurons in the monkey superior colliculus predict the visual result of impending saccadic eye movements. Journal of neurophysiology. 1995; 73(5):1988-2003. PMID: 7623096

7. Wurtz RH, Joiner WM, Berman RA. Neuronal mechanisms for visual stability: progress and problems. Philosophical transactions of the Royal Society of London Series B, Biological sciences. 2011; 366 (1564):492-503. doi: 10.1098/rstb.2010.0186 PMID: 21242138

8. Cavanagh P, Hunt AR, Afraz A, Rolfs M. Visual stability based on remapping of attention pointers. Trends in cognitive sciences. 2010; 14(4):147-53. doi: 10.1016/j.tics.2010.01.007 PMID: 20189870

9. Melcher $\mathrm{D}$. The missing link for attention pointers: comment on Cavanagh et al. Trends in cognitive sciences. 2010; 14(11):473; author reply 4-5. doi: 10.1016/j.tics.2010.08.007 PMID: 20851665

10. Melcher D, Colby CL. Trans-saccadic perception. Trends in cognitive sciences. 2008; 12(12):466-73. doi: 10.1016/j.tics.2008.09.003 PMID: 18951831

11. Mayo JP, Sommer MA. Shifting attention to neurons. Trends in cognitive sciences. 2010; 14(9):389; author reply 90-1. doi: 10.1016/j.tics.2010.06.003 PMID: 20591722

12. Crapse TB, Sommer MA. Frontal eye field neurons assess visual stability across saccades. The Journal of neuroscience: the official journal of the Society for Neuroscience. 2012; 32(8):2835-45. 
13. Ong WS, Bisley JW. A lack of anticipatory remapping of retinotopic receptive fields in the middle temporal area. The Journal of neuroscience: the official journal of the Society for Neuroscience. 2011; 31 (29):10432-6.

14. Busse L, Katzner S, Treue S. Temporal dynamics of neuronal modulation during exogenous and endogenous shifts of visual attention in macaque area MT. Proceedings of the National Academy of Sciences of the United States of America. 2008; 105(42):16380-5. doi: 10.1073/pnas.0707369105 PMID: 18922778

15. Katzner S, Busse L, Treue S. Attention to the Color of a Moving Stimulus Modulates Motion-Signal Processing in Macaque Area MT: Evidence for a Unified Attentional System. Frontiers in systems neuroscience. 2009; 3:12. doi: 10.3389/neuro.06.012.2009 PMID: 19893762

16. Treue S, Maunsell JH. Attentional modulation of visual motion processing in cortical areas MT and MST. Nature. 1996; 382(6591):539-41. PMID: 8700227

17. Zaksas D, Pasternak T. Area MT neurons respond to visual motion distant from their receptive fields. Journal of neurophysiology. 2005; 94(6):4156-67. PMID: 16120662

18. Treue S, Martinez Trujillo JC. Feature-based attention influences motion processing gain in macaque visual cortex. Nature. 1999; 399(6736):575-9. PMID: 10376597

19. Ibbotson MR, Crowder NA, Cloherty SL, Price NS, Mustari MJ. Saccadic modulation of neural responses: possible roles in saccadic suppression, enhancement, and time compression. The Journal of neuroscience: the official journal of the Society for Neuroscience. 2008; 28(43):10952-60.

20. Cloherty SL, Mustari MJ, Rosa MG, Ibbotson MR. Effects of saccades on visual processing in primate MSTd. Vision research. 2010; 50(24):2683-91. doi: 10.1016/j.visres.2010.08.020 PMID: 20732345

21. Blatt GJ, Andersen RA, Stoner GR. Visual receptive field organization and cortico-cortical connections of the lateral intraparietal area (area LIP) in the macaque. The Journal of comparative neurology. 1990; 299(4):421-45. PMID: 2243159

22. Maunsell JH, van Essen DC. The connections of the middle temporal visual area (MT) and their relationship to a cortical hierarchy in the macaque monkey. The Journal of neuroscience: the official journal of the Society for Neuroscience. 1983; 3(12):2563-86.

23. Ungerleider LG, Desimone R. Cortical connections of visual area MT in the macaque. The Journal of comparative neurology. 1986; 248(2):190-222. PMID: 3722458

24. Price NS, Ibbotson MR, Ono S, Mustari MJ. Rapid processing of retinal slip during saccades in macaque area MT. Journal of neurophysiology. 2005; 94(1):235-46. PMID: 15772244

25. Yeh $\mathrm{Cl}$, Xing D, Shapley RM. "Black" responses dominate macaque primary visual cortex 1 1. The Journal of neuroscience: the official journal of the Society for Neuroscience. 2009; 29(38):11753-60.

26. Jonikaitis D, Szinte M, Rolfs M, Cavanagh P. Allocation of attention across saccades. Journal of neurophysiology. 2013; 109(5):1425-34. doi: 10.1152/jn.00656.2012 PMID: 23221410

27. Rolfs $M$, Jonikaitis $D$, Deubel $H$, Cavanagh $P$. Predictive remapping of attention across eye movements. Nature neuroscience. 2011; 14(2):252-6. doi: 10.1038/nn.2711 PMID: 21186360

28. Szinte $M$, Carrasco $M$, Cavanagh $P$, Rolfs $M$. Attentional trade-offs maintain the tracking of moving objects across saccades. Journal of neurophysiology. 2015; 113(7):2220-31. doi: 10.1152/jn.00966. 2014 PMID: 25609111

29. Mirpour K, Bisley JW. Anticipatory remapping of attentional priority across the entire visual field. The Journal of neuroscience: the official journal of the Society for Neuroscience. 2012; 32(46):16449-57.

30. Gottlieb JP, Kusunoki M, Goldberg ME. The representation of visual salience in monkey parietal cortex. Nature. 1998; 391(6666):481-4. PMID: 9461214

31. Joiner WM, Cavanaugh J, Wurtz $\mathrm{RH}$. Modulation of shifting receptive field activity in frontal eye field by visual salience. Journal of neurophysiology. 2011; 106(3):1179-90. doi: 10.1152/jn.01054.2010 PMID: 21653709

32. Zirnsak M, Moore T. Saccades and shifting receptive fields: anticipating consequences or selecting targets? Trends in cognitive sciences. 2014; 18(12):621-8. doi: 10.1016/j.tics.2014.10.002 PMID: 25455690

33. Zirnsak M, Steinmetz NA, Noudoost B, Xu KZ, Moore T. Visual space is compressed in prefrontal cortex before eye movements. Nature. 2014; 507(7493):504-7. doi: 10.1038/nature13149 PMID: 24670771

34. Hoffman JE, Subramaniam B. The role of visual attention in saccadic eye movements. Perception \& psychophysics. 1995; 57(6):787-95.

35. Kowler E, Anderson E, Dosher B, Blaser E. The role of attention in the programming of saccades. Vision research. 1995; 35(13):1897-916. PMID: 7660596 
36. Hallett PE, Lightstone AD. Saccadic eye movements towards stimuli triggered by prior saccades. Vision research. 1976; 16(1):99-106. PMID: 1258395

37. McPeek RM, Skavenski AA, Nakayama K. Concurrent processing of saccades in visual search. Vision research. 2000; 40(18):2499-516. PMID: 10915889

38. Phillips AN, Segraves MA. Predictive activity in macaque frontal eye field neurons during natural scene searching. Journal of neurophysiology. 2010; 103(3):1238-52. doi: 10.1152/jn.00776.2009 PMID: 20018833

39. Mays LE, Sparks DL. Dissociation of visual and saccade-related responses in superior colliculus neurons. Journal of neurophysiology. 1980; 43(1):207-32. PMID: 6766178

40. Goldberg ME, Bruce CJ. Primate frontal eye fields. III. Maintenance of a spatially accurate saccade signal. Journal of neurophysiology. 1990; 64(2):489-508. PMID: 2213128

41. Barash S, Bracewell RM, Fogassi L, Gnadt JW, Andersen RA. Saccade-related activity in the lateral intraparietal area. II. Spatial properties. Journal of neurophysiology. 1991; 66(3):1109-24. PMID: 1753277

42. Goldberg ME, Colby CL, Duhamel JR. Representation of visuomotor space in the parietal lobe of the monkey. Cold Spring Harbor symposia on quantitative biology. 1990; 55:729-39. PMID: 2132850

43. Mays LE, Sparks DL. Saccades are spatially, not retinocentrically, coded. Science. 1980; 208 (4448):1163-5. PMID: 6769161

44. Sparks DL, Porter JD. Spatial localization of saccade targets. II. Activity of superior colliculus neurons preceding compensatory saccades. Journal of neurophysiology. 1983; 49(1):64-74. PMID: 6827304

45. Wurtz RH. Neuronal mechanisms of visual stability. Vision research. 2008; 48(20):2070-89. doi: 10. 1016/j.visres.2008.03.021 PMID: 18513781

46. Ibbotson $\mathrm{M}$, Krekelberg B. Visual perception and saccadic eye movements. Current opinion in neurobiology. 2011; 21(4):553-8. doi: 10.1016/j.conb.2011.05.012 PMID: 21646014

47. Subramanian J, Colby CL. Shape selectivity and remapping in dorsal stream visual area LIP. Journal of neurophysiology. 2014; 111(3):613-27. doi: 10.1152/jn.00841.2011 PMID: 24225538

48. Deubel H, Schneider WX. Saccade target selection and object recognition: evidence for a common attentional mechanism. Vision research. 1996; 36(12):1827-37. PMID: 8759451

49. Montagnini A, Castet E. Spatiotemporal dynamics of visual attention during saccade preparation: Independence and coupling between attention and movement planning. Journal of vision. 2007; 7(14):8 1-16. doi: 10.1167/7.14.8 PMID: 18217803

50. Zhao M, Gersch TM, Schnitzer BS, Dosher BA, Kowler E. Eye movements and attention: the role of pre-saccadic shifts of attention in perception, memory and the control of saccades. Vision research. 2012; 74:40-60. doi: 10.1016/j.visres.2012.06.017 PMID: 22809798 


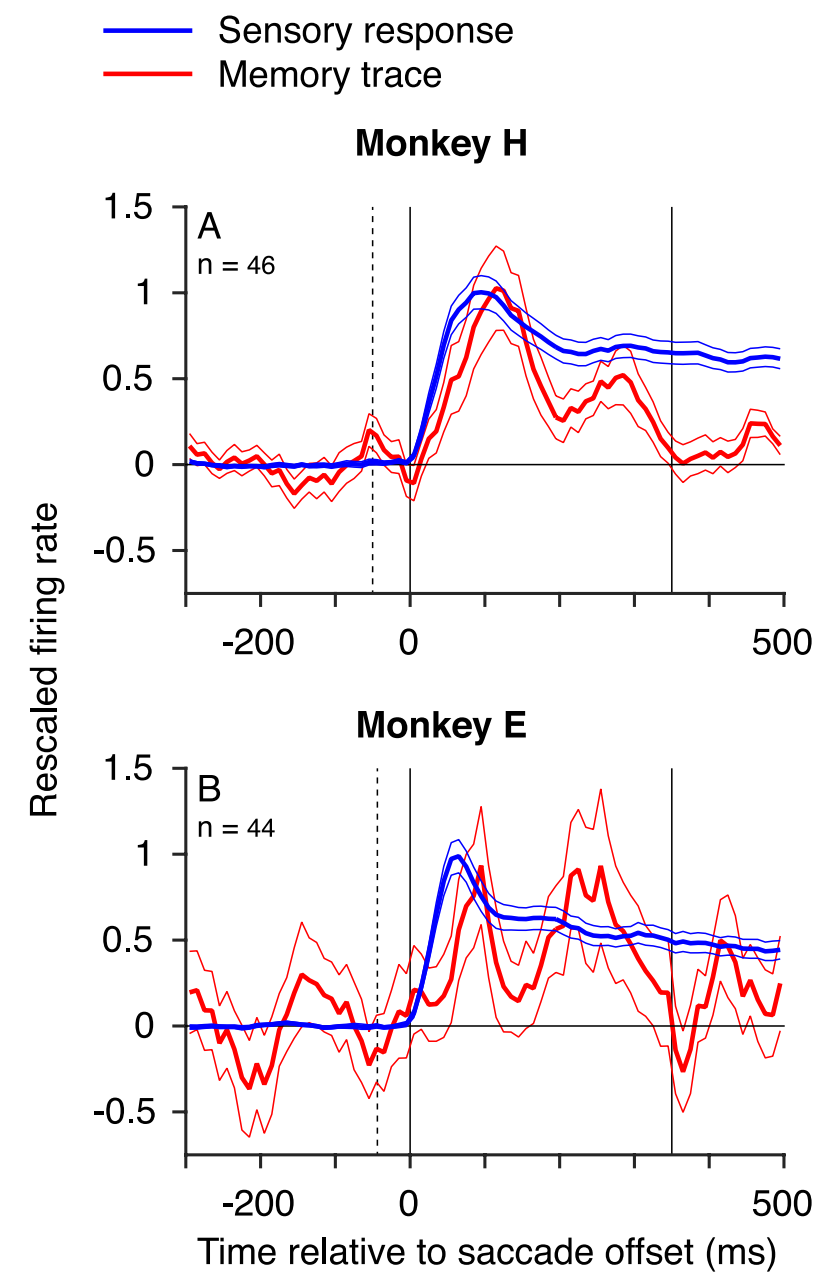

Figure S1: The memory trace does not start earlier than the sensory

response. The memory trace, plotted as the difference between the response in the attend-in condition of the interrupted stimulus task and the response in the simplestimulus task (mean difference across neurons and SEM - red trace), arises at the same time or later than the sensory response, plotted as the difference between the response in the continuous stimulus task with the preferred direction and the response in the simple-stimulus task (mean difference across neurons and SEM blue trace). The contribution of predictive remapping to the timing of the steep rise of the sensory response towards its peak would be minimal, and the memory trace does not appear to lead the sensory response anywhere along this steep rise. In order to facilitate comparison, both traces were normalized by subtracting the mean value of the trace from -300 to $0 \mathrm{~ms}$, and then dividing by the maximum value. Data for monkey $\mathrm{H}(\mathrm{A})$ and monkey $\mathrm{E}(\mathrm{B})$. Other conventions as in Fig 2. Data in Supporting Information (S6 Data). 


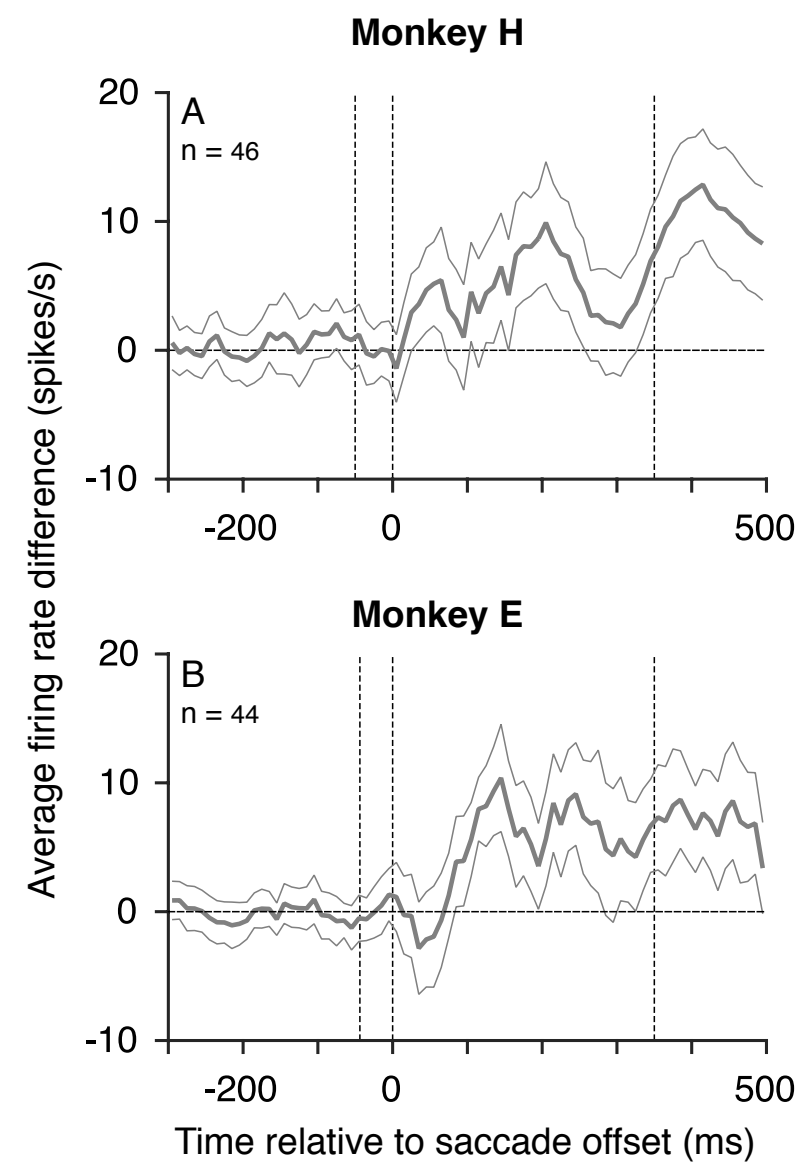

Figure S2: Attentional effects in the continuous task manifest at or after saccade offset. The attentional effect in the continuous task for the preferred direction, plotted as the difference between the responses in the attend-in (Fig 2, blue curve) and attend-out (Fig 2, red curve) conditions (mean and $95 \%$ confidence bands), rises above zero only after saccade offset. Data for monkey $H(A)$ and monkey $E$ (B). Other conventions as in Fig 2. Data in Supporting Information (S7 Data). 


\section{Supporting information}

Methods

This is an expanded version of the Methods section in the main text. We trained two male rhesus monkeys (Macaca mulatta, $7-11 \mathrm{~kg}$ ), monkey $\mathrm{H}$ and monkey $\mathrm{E}$, to perform a demanding visuospatial-attention task along with a saccade. Each monkey was implanted with a titanium head holder to minimize head movements during the experiment. One recording chamber was also implanted in each monkey above the left (monkey E) or the right (monkey $\mathrm{H}$ ) parietal cortex to allow access to MT, with implantation locations chosen based on a preceding MRI scan. All procedures were approved by the district government of Lower Saxony, Germany, and all surgeries were conducted under general anesthesia using standard techniques.

The experiments were performed in a dimly-lit room with the only source of light being the display monitor. A CRT monitor (Sony Trinitron GDM-FW900) at a distance of $57 \mathrm{~cm}$ from the monkey was used to display the visual stimulus at a refresh rate of $76 \mathrm{~Hz}$ and a spatial resolution of 40 pixels/degree. The monkey sat in a custom-made primate chair during the experiment. Stimulus presentation, reward delivery, electrophysiological and behavioral data collection was controlled by custom software and run on an Apple Macintosh computer. All stimulus onsets and durations were specified in terms of number of frames (CRT monitor refreshes), and the times reported here in millisecond units are correct to within $13 \mathrm{~ms}$ (the duration of one frame), given the vertical scan-rate properties of the CRT monitor. The animals received a fluid reward immediately following each correct trial. The eye-position was monitored by an EyeLink 1000 (SR Research, Canada) system at $1000 \mathrm{~Hz}$. Neuronal activity was recorded extracellularly with a 5channel micro drive system (Mini Matrix, Thomas Recording, Giessen, Germany) and processed using the Plexon data acquisition system (Plexon Inc., Dallas, TX). Only data from well-isolated neurons were used for the analysis. MT was identified by referencing the recordings to the structural $\mathrm{MRI}$ and by the physiological properties of the recorded neurons: most 
neurons were direction-tuned, the average diameter of the receptive fields (RFs) was approximately equal to the RF eccentricity and there was a predictable progression of RF centers at different locations along the superior temporal sulcus.

\section{Behavioral tasks and stimuli}

Once a neuron was isolated and while the monkey performed a fixation task, we located the RF by moving a stationary circular random dot pattern (RDP) across the screen using a mouse. During this task, the monkey had to maintain fixation on a fixation point and respond to a brief luminance change at the fixation point. We then determined the neuron's preferred direction and speed, again while the monkey performed a fixation task (usually at the location of the saccade target in the main task), by presenting a RDP with moving dots within a circular aperture in the RF, changing the direction and speed every $250 \mathrm{~ms}$ picked from a set of 3 possible speeds $(4,8$ or 16 degrees per second) and 12 possible directions (evenly separated by 30 degrees around a circle). The preferred and anti-preferred directions, and the preferred speed were used in the main experiment. Occasionally, we recorded simultaneously from two or more neurons with overlapping RFs when their preferred directions also overlapped or were opposite to each other.

After identifying the RF location and preferred direction, we switched to the main experiment. In the main experiment (Figure 1), each trial was composed of one of four tasks (three experimental tasks and one control task, chosen in a pseudo-randomly interleaved manner). For all four tasks, the monkeys initiated the trial by holding a lever and foveating a black fixation point. In the control task (the "simple-saccade" task), performed on $11.1 \%$ of trials, a saccade target, identical to the fixation point, appeared between 10 and 20 degrees eccentrically (value fixed for each neuron, and either 15 or 20 degrees in most cases), $1382 \mathrm{~ms}$ after the monkey initiated fixation. The saccade target then stayed on for $1368 \mathrm{~ms}$ (the end of the trial). Saccades were always either horizontal or vertical. In all tasks, there was a 
one-frame $(13 \mathrm{~ms})$ overlap between the fixation point and the saccade target, so that the fixation point disappeared one frame after the saccade target appeared: perceptually, the fixation point appeared to jump from its original location to the saccade target. Once the fixation point jumped, the monkey had to make a saccade to the new location of the fixation point within $263 \mathrm{~ms}$ and maintain fixation until the end of the trial in order to obtain a reward for correct performance. In the three experimental tasks, after $118 \mathrm{~ms}$ of fixation, a stationary RDP cue within a circular aperture (of the same size as the target), which indicated the location of the future target of attention, was presented for $263 \mathrm{~ms}$ either in the neurons' post-saccadic RF (attend-in condition) or opposite to it (attend-out condition) equally often. After an additional delay of 329 ms following cue offset, two moving RDPs (2 degrees in radius, all dots moving in the same direction of motion and within stationary circular apertures) were presented on the screen, with both moving equally often in the neurons' preferred or anti-preferred direction. One of them, the target was located at the previously cued location, while the other, the distractor, was located opposite to it (i.e. reflected across the horizontal or vertical meridian, see Figure 1). The monkeys' task was to respond to a brief (132 $\mathrm{ms}$ ) direction change in the target by releasing the lever (within $600 \mathrm{~ms}$ of the change), but ignore similar changes in the distractor. In addition, during the trial, if the fixation point jumped to a new location (as in the "simple-saccade" task), the monkeys had to refixate the fixation point while continuing to attend to the cued target. In the first of the three experimental tasks (the "continuous-stimulus task", $22.2 \%$ of trials), the fixation point jumped to its new location $671 \mathrm{~ms}$ after RDP onset. The direction change in the target RDP could occur between $974 \mathrm{~ms}$ to $1895 \mathrm{~ms}$ after the fixation point jumped. The second experimental task (the "interrupted-stimulus task", $44.4 \%$ of trials) was similar to the continuousstimulus task, and the fixation point jumped at the same time as in the continuous-stimulus task, but the target and distractor RDPs disappeared $105 \mathrm{~ms}$ after the fixation point jumped. Therefore, on these trials, no stimulus ever appeared in the neurons' RF after the saccade (or before the saccade). The monkeys had to simply make a saccade to the new fixation point location and maintain fixation until the end of the trial to obtain a reward. The 
few trials with saccades that started before the disappearance of the stimulus were discarded. The third experimental task (the "fixation task", $22.2 \%$ of trials) was also similar to the continuous-stimulus task, except that the fixation point never jumped, and the direction-change in the target RDP occurred $789 \mathrm{~ms}$ to $1842 \mathrm{~ms}$ after RDP onset. This task was included to make sure the monkeys paid attention to the target even during the time when they made a saccade in the other two experimental tasks, and was not analyzed further for this study.

In all the tasks, the background was always grey with a luminance of 14.2 $\mathrm{cd} / \mathrm{m}^{2}$, and the fixation point and RDPs including the stationary cue were black with the luminance of $0.68 \mathrm{~cd} / \mathrm{m}^{2}$. Our use of black stimuli minimizes the concerns that arise when white stimuli are used on a black background regarding the persistence of visual stimuli on the display monitor after their stipulated disappearance. Individual RDP dot size was $0.1^{\circ} \times 0.1^{\circ}$, and the dot density was $10 \mathrm{dots} / \mathrm{deg}^{2}$. Monkeys had to maintain fixation within a circular window of 2 degrees radius around the fixation point, except for a period of $263 \mathrm{~ms}$ after the fixation point jumped to give them time to make the saccade. The saccade direction was set according to the position of the RF: for example, if the RF center was directly above or below the fixation point, we used a horizontal saccade, while if the RF center was directly to the left or right of the fixation point, we used a vertical saccade. If the RF center was offset both vertically and horizontally from the fixation point, the choice was no longer critical, but we usually used a horizontal saccade.

Data analysis

We only included correctly performed trials in our analysis. After excluding fixation breaks, both monkeys performed the tasks correctly on over $94.6 \%$ of trials. Data analysis was performed using custom software in MATLAB (MATLAB Inc, Natick, MA). We included data from all neurons that showed a 
significantly greater post-saccadic response to at least one of the two directions in the continuous-stimulus task (compared to the simple saccade task where there is no stimulus in the RF, i.e. they were visually responsive to the RDP) as well as a significant difference between the responses to the two RDP directions in the continuous-stimulus task (i.e. they showed direction tuning). Additionally, we excluded neurons where the onset of the RDP at the (future) post-saccadic RF location elicited a statistically significant response from the neuron. Peri-stimulus time histograms (PSTHs: Figures 24) were calculated using partially overlapping bins (50 ms width, stepped every $10 \mathrm{~ms}$ ): the mean activity for each neuron across trials was first calculated and then these mean PSTHs for individual neurons were averaged across neurons to obtain the displayed PSTHs. For the differences between conditions (say $A$ and $B$ ), we report the effects using a modulation index, which is defined in the usual manner for each neuron as the difference in the firing-rates for the two conditions divided by their sum. A directiontuning index was similarly defined as the difference in firing-rates for preferred and anti-preferred directions divided by their sum (Figure 5). We report the average modulation and direction-tuning indices using the median value after converting it back into a percentage. For the analyses of the memory trace in Figures 3 and 4, because we did not find a difference between the response in the interrupted-stimulus task based on whether the RDP was moving in the preferred or anti-preferred direction before the saccade (Figure 5), we pooled the trials irrespective of RDP direction and only separated the trials into those where the monkey was cued to attend to the post-saccadic RF location (attend-in condition) and those where the monkey was cued to attend outside it (attend-out condition).

We detected saccades using a standard velocity-threshold algorithm: onset (and offset) times were determined by when the eye velocity exceeded (and then dropped below) 30 degrees per second. This threshold value was set to lie clearly above the peak excursions of the baseline noise in the eye-velocity traces, and the algorithm was validated by visual inspection for each monkey. In addition to the large saccade from the fixation point to the saccade target (to which we aligned our data for our analyses), both 
monkeys also made small-amplitude eye-movements within the fixation window while fixating: these eye-movements could be observed to induce a short-duration transient response lasting less than $100 \mathrm{~ms}$ when there was a motion stimulus in the RF [as has been reported earlier: 1]. For the main analyses of interest in this paper, there was no stimulus in the RF after the saccade and no transient response was detected following the microsaccade. Even so, we re-examined our results and conclusions after discarding spikes elicited within $100 \mathrm{~ms}$ after any small eye movement occurring after the large refixation saccade, within the time-windows of interest. This correction was made both for the PSTHs and for the statistical comparisons based on spike-counts. We verified that our results remain similar and that our conclusions remain robust when such a correction for small eye-movements is made.

1. Herrington TM, Masse NY, Hachmeh KJ, Smith JE, Assad JA, Cook EP. The effect of microsaccades on the correlation between neural activity and behavior in middle temporal, ventral intraparietal, and lateral intraparietal areas. The Journal of neuroscience : the official journal of the Society for Neuroscience. 2009;29(18):5793-805. 


\section{Additional supplementary materials 1: Example eye traces}

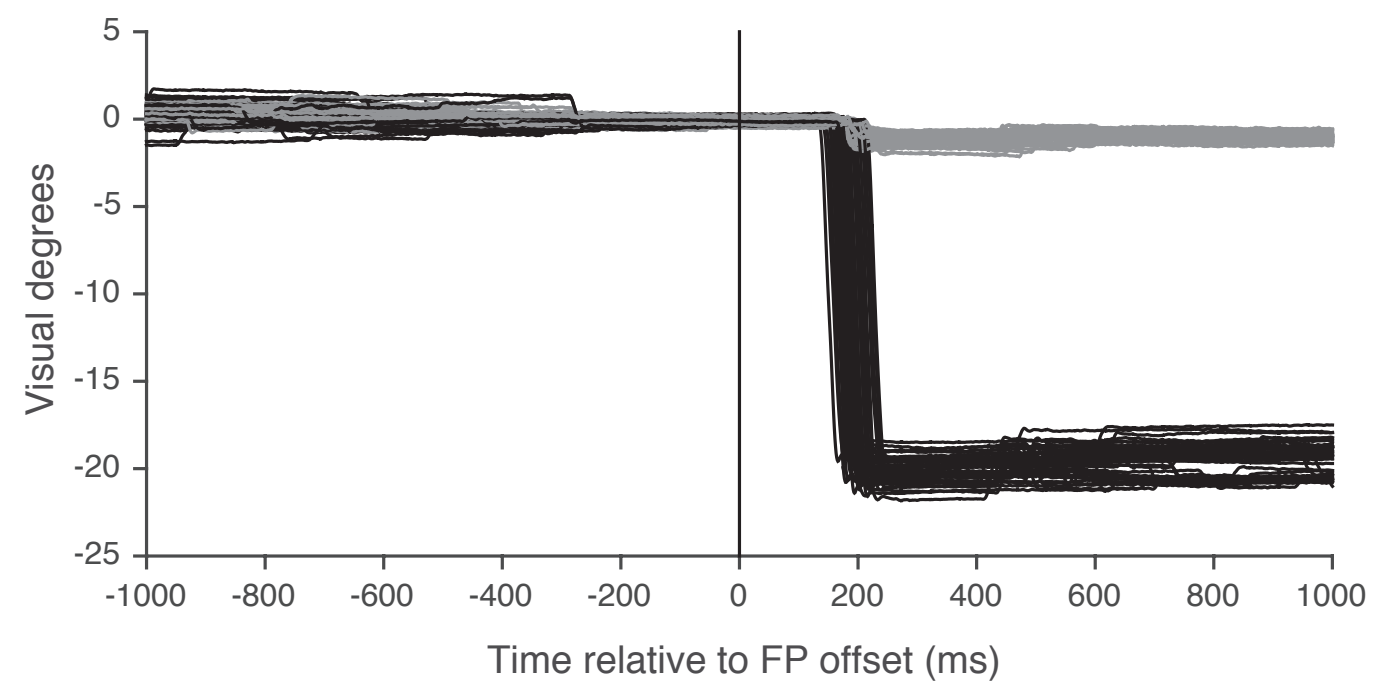

The example eye traces of trial No. 80-180 from Monkey E in the experiment when he was performing the task and made a sacccade in that trial. The black and gray traces indicate the horizontal and vertical eye position respectively. In this session, the animal was required to perform a 20 visual angle degrees saccade to the left when he saw the fixation point jump. (recording session: tao-trAttC-edg-047-001+02). 


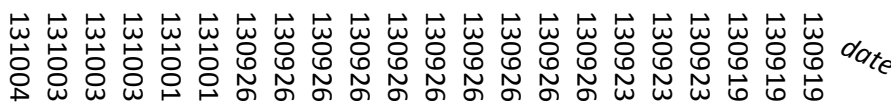

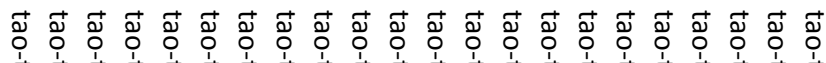

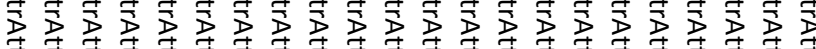

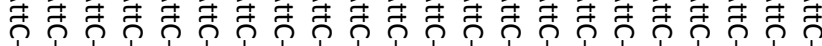

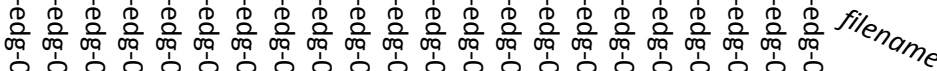

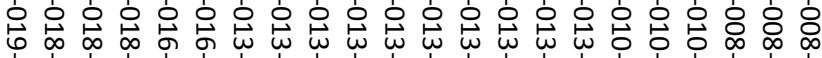

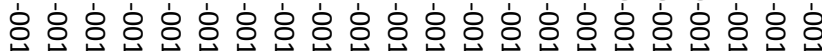

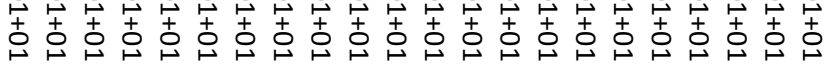

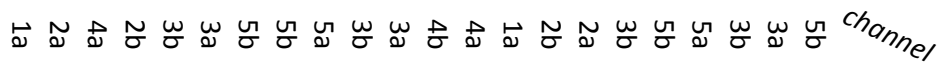

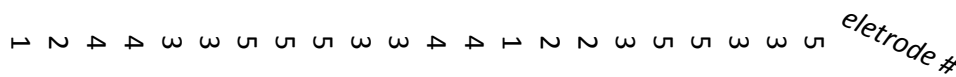

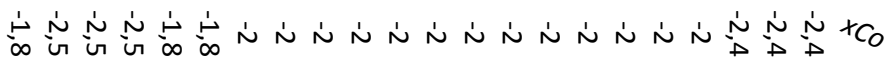

$\dot{\nu}$ نั

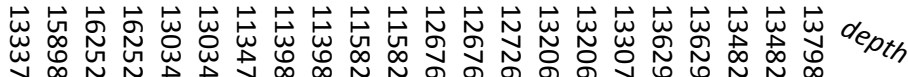

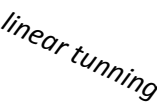

$\begin{array}{llllllllllllllllll} & \infty & \infty & \infty & \infty & \infty & \infty & \infty & \infty & \infty & \infty & \infty & \infty & \infty & 0 & \infty & \infty & \text { shal }_{a}\end{array}$

$0000000000000000000000^{\text {spiraltuning }}$

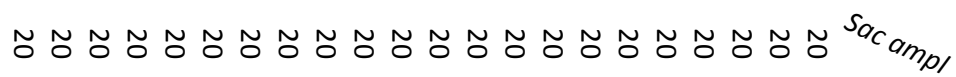

○

蟫

のち。

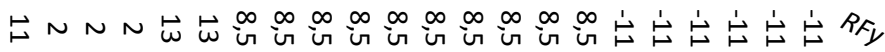

$0000000000000000000000^{\text {Pisize }}$

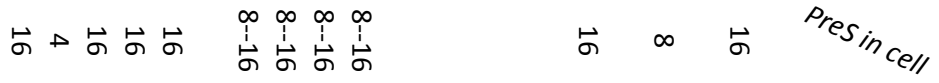

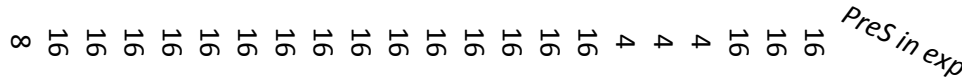

동 잉 잉

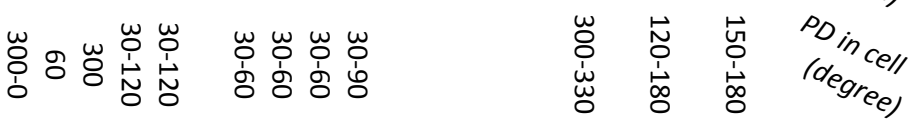

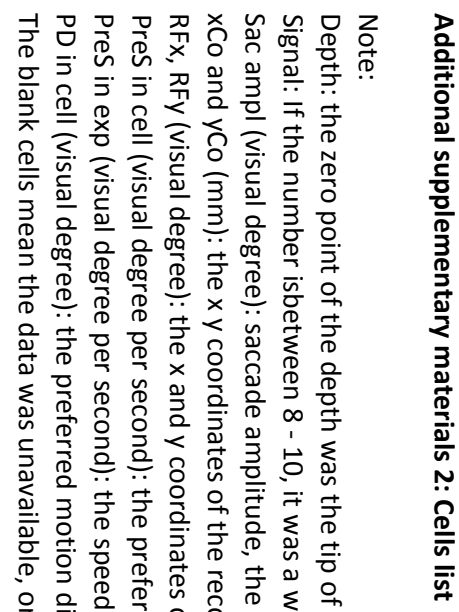

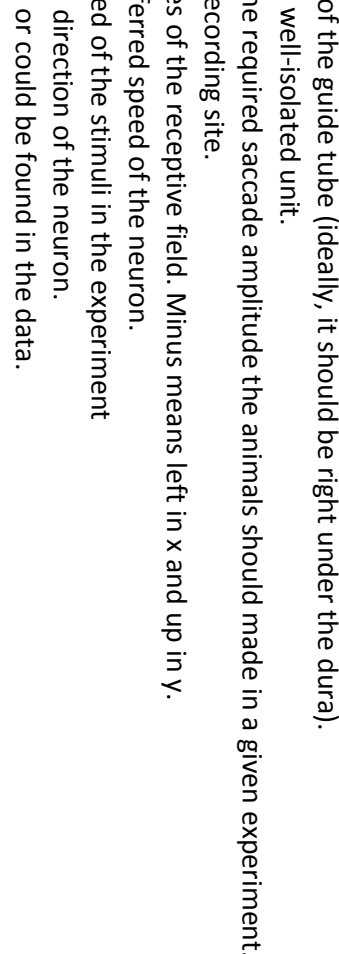




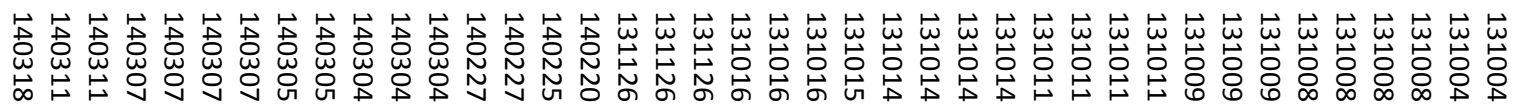

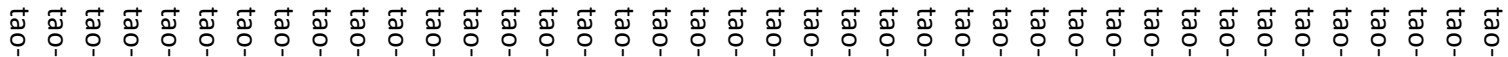

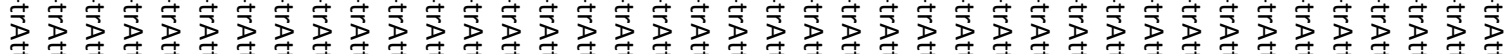

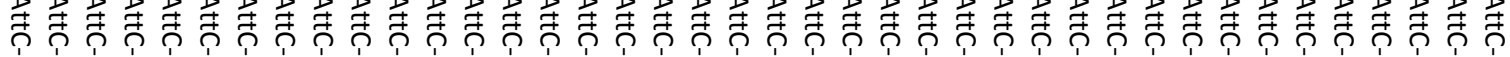

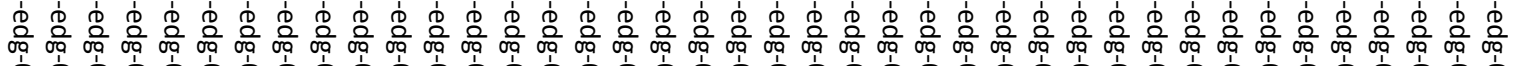

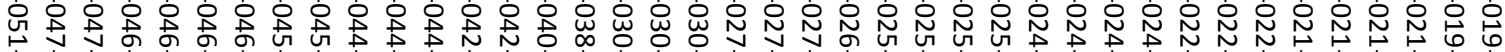

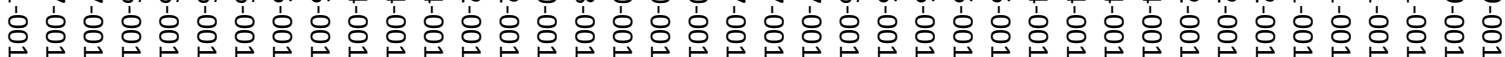

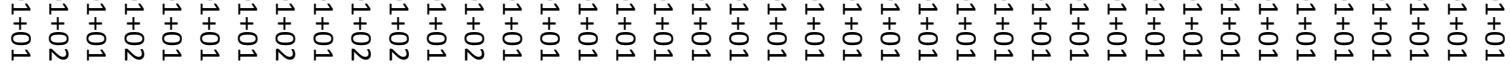

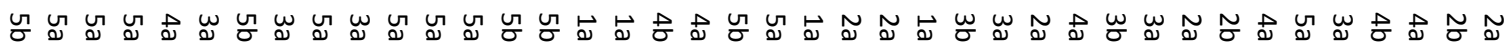

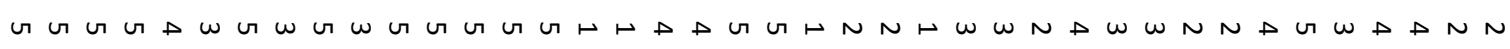

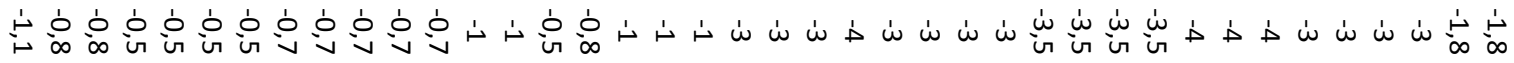

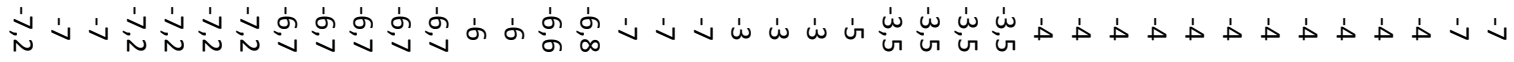

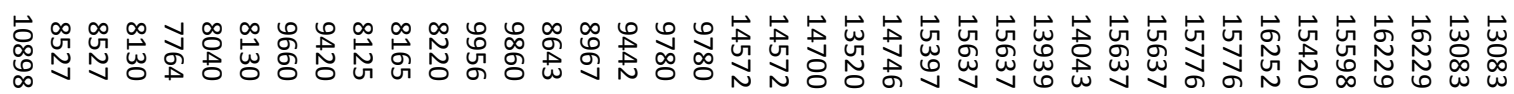

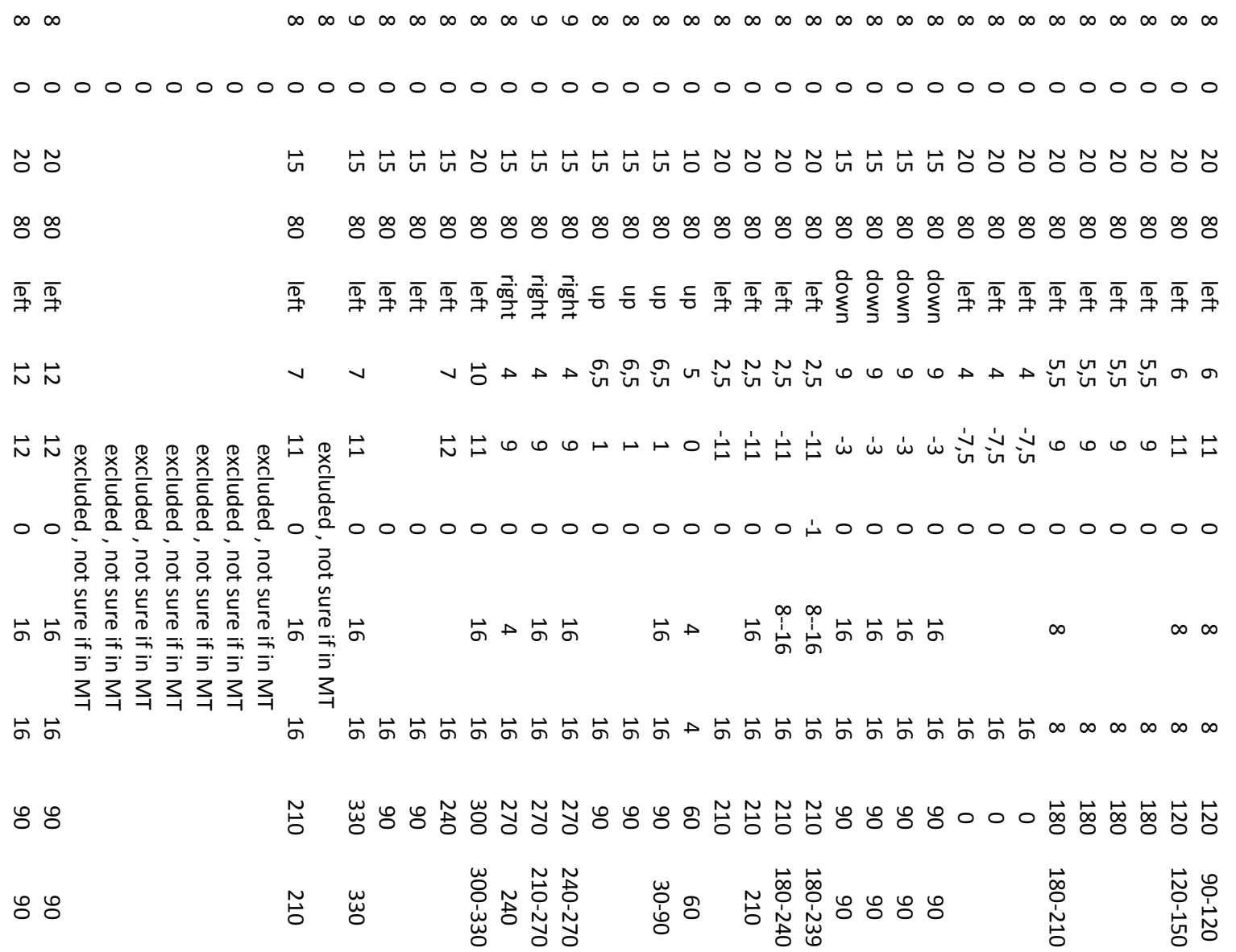




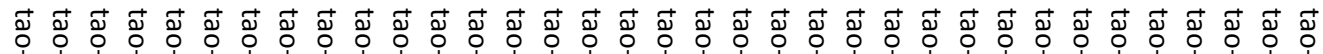

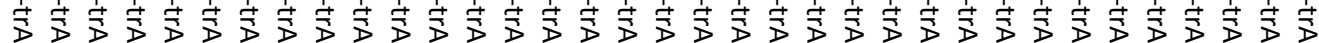

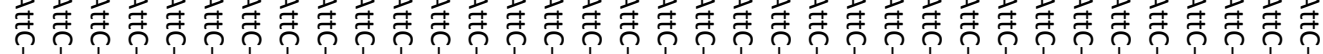

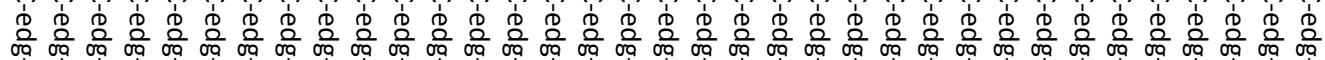

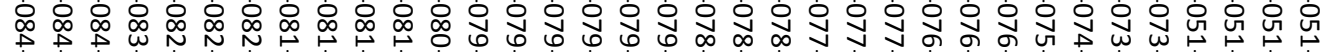

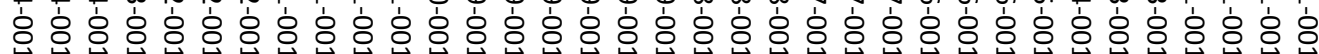

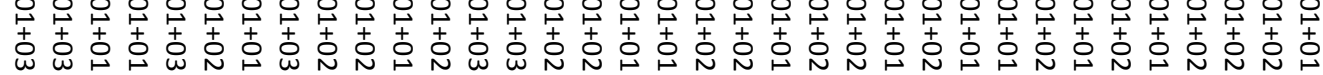

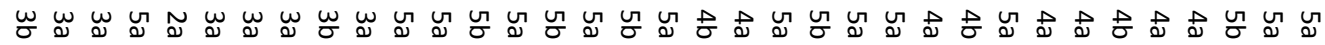

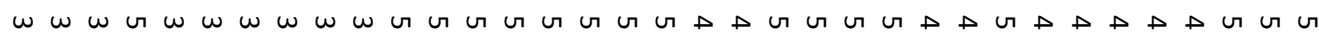

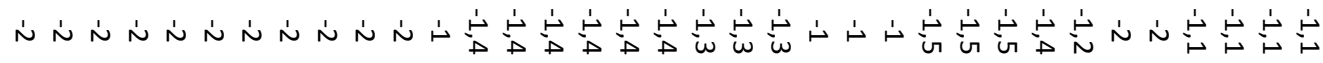

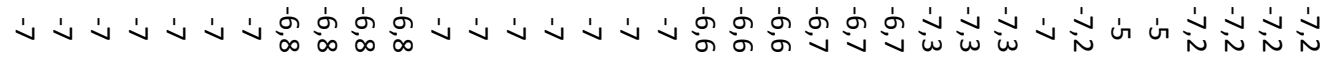

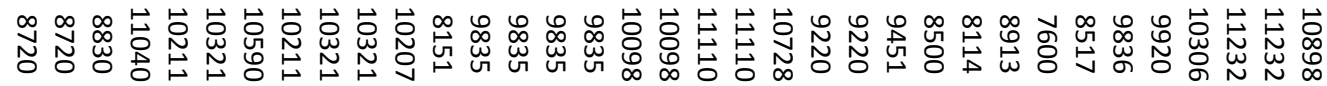
$\stackrel{\infty}{\circ} \stackrel{\infty}{\circ} \stackrel{\infty}{\circ} \stackrel{\infty}{\circ} \stackrel{\infty}{\circ} \stackrel{\infty}{\circ} \stackrel{\infty}{\circ} \stackrel{\infty}{\circ} \stackrel{\infty}{\circ} \stackrel{\infty}{\circ}$ $\stackrel{\infty}{\circ} \stackrel{\infty}{\circ} \stackrel{\infty}{\circ} \stackrel{\infty}{\circ}$

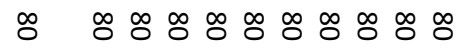
등 $\frac{\text { oำ }}{\frac{7}{7}}$

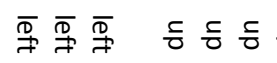
$\stackrel{\overline{0}}{\rightrightarrows} \stackrel{\overline{0}}{\rightrightarrows} \stackrel{\overline{0}}{\rightleftarrows}$ $\stackrel{\bar{D}}{\rightrightarrows} \stackrel{\overline{0}}{\rightrightarrows}$ 등 등 둥

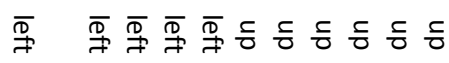
它官 $\omega \omega \omega$ เ $w \quad$ v $v$

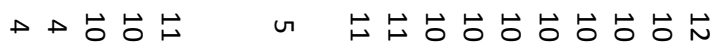

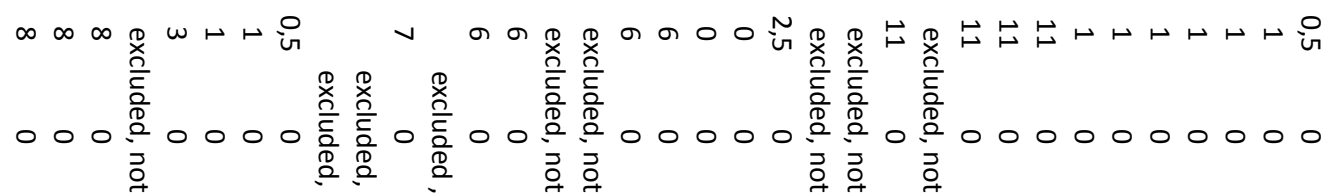

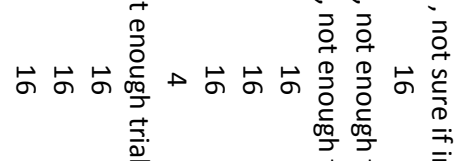

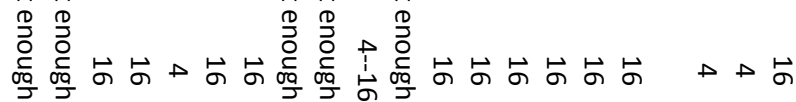

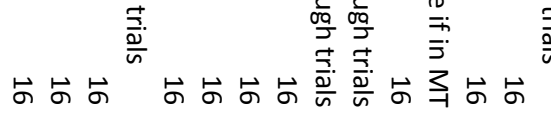

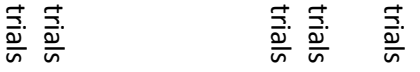

㟧

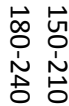

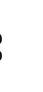

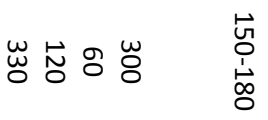

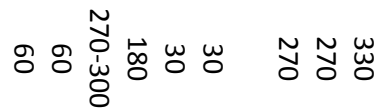




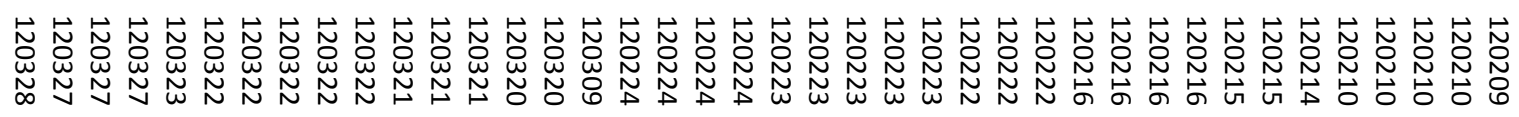

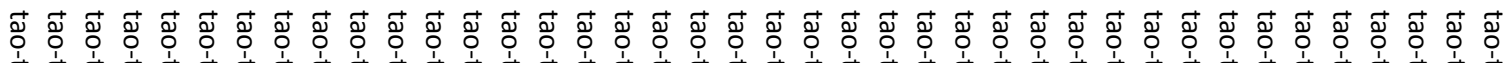

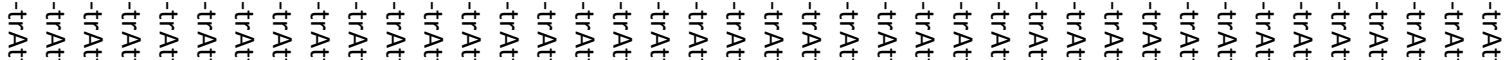

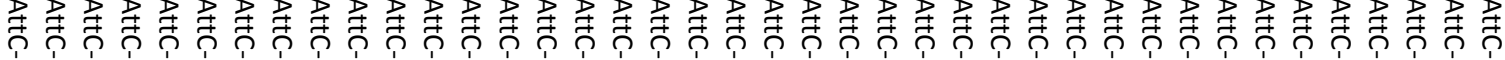

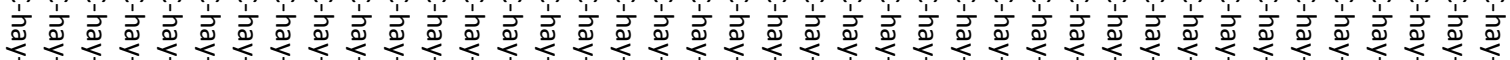

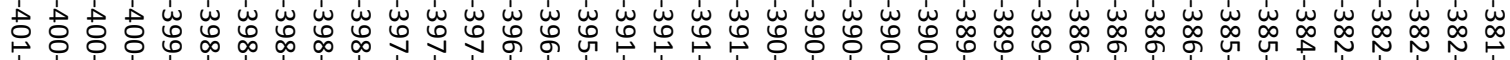

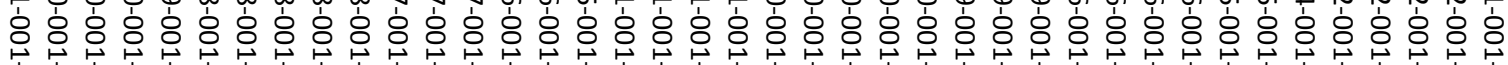

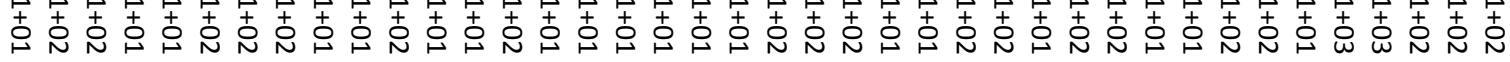

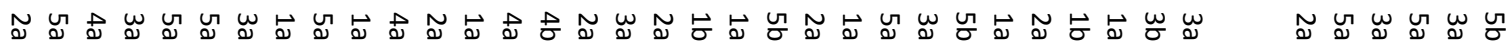

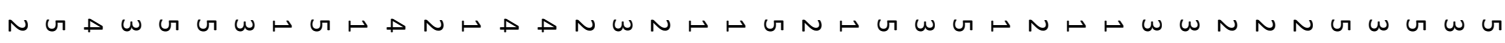

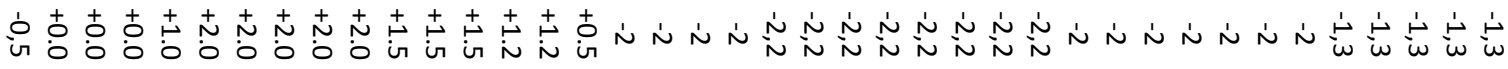

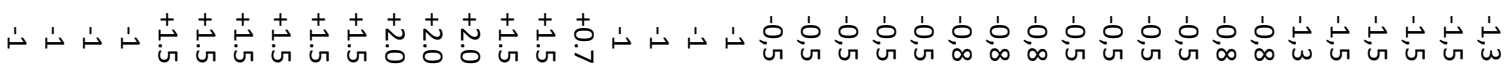

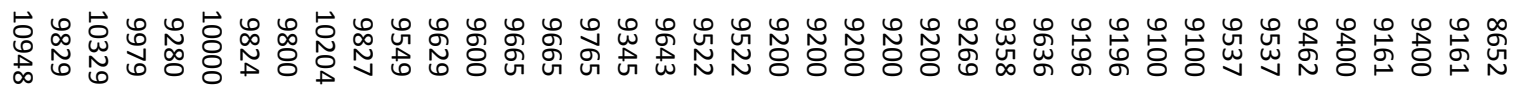

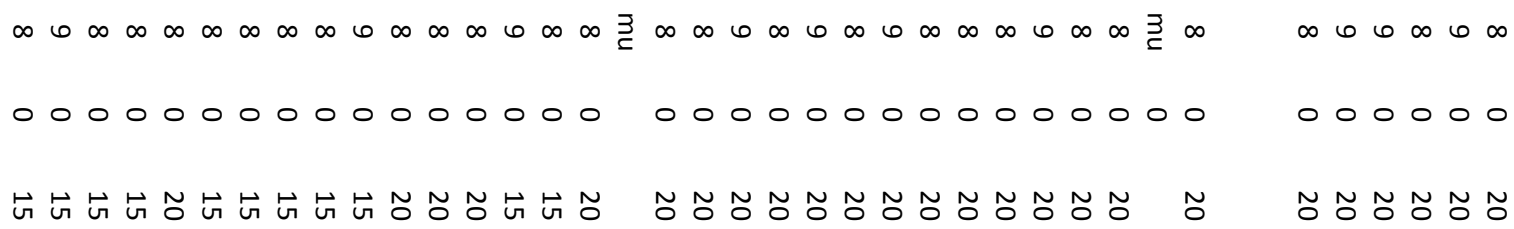

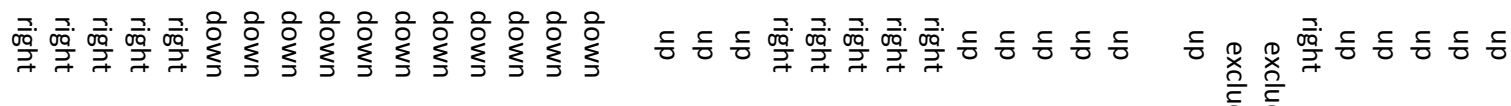

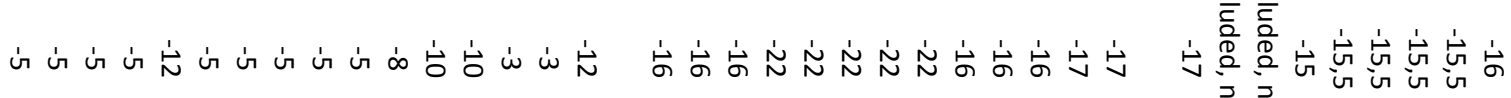

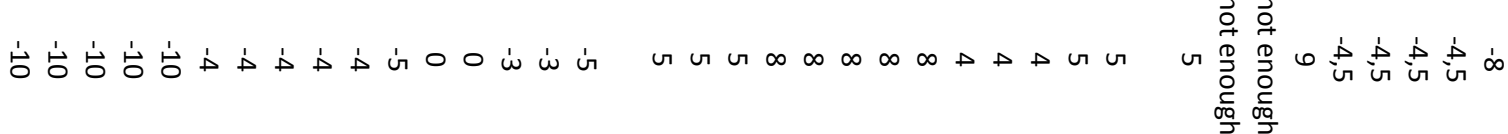

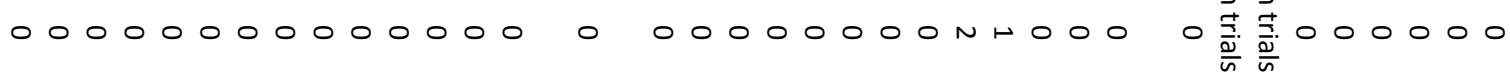

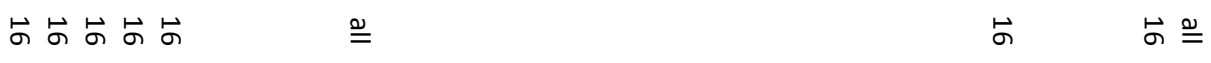

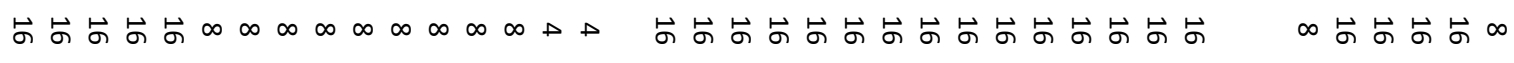

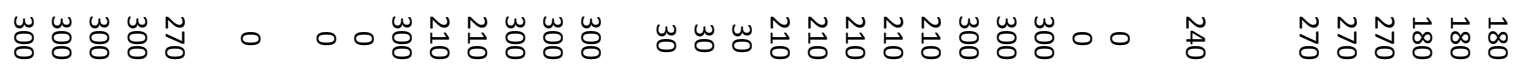

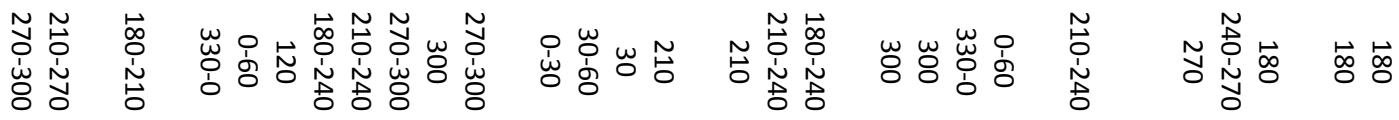




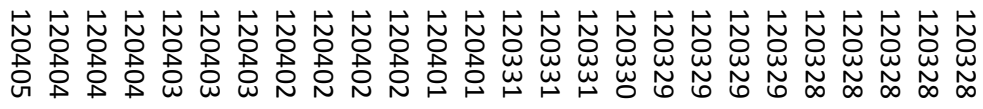

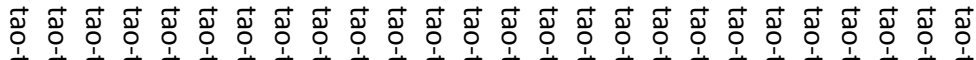

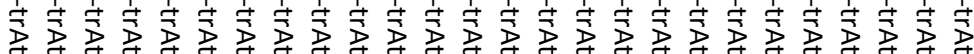

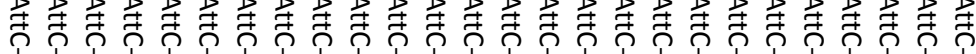

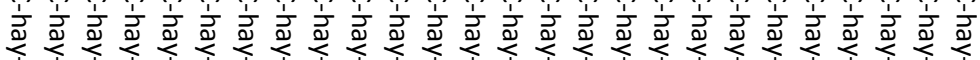

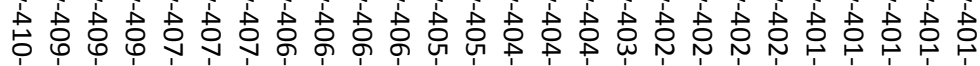

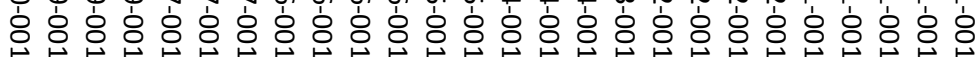

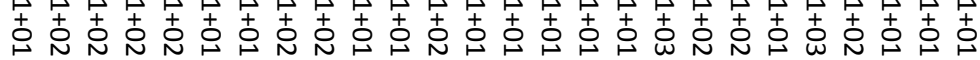

จ

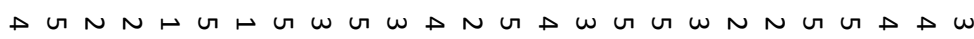

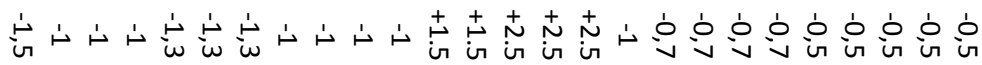

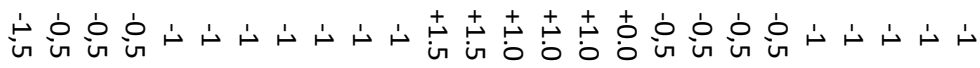

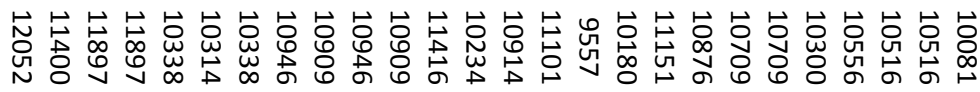

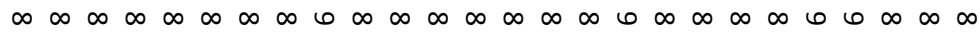
00000000000000000000000000

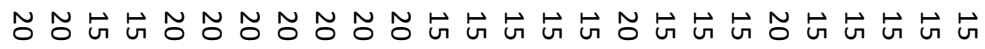

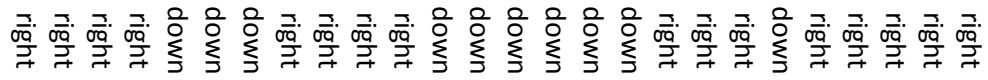

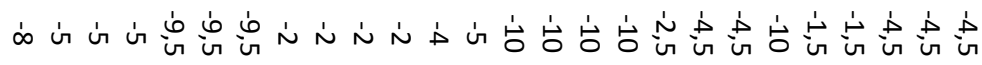

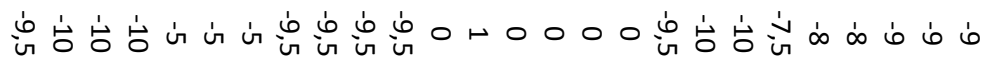
00000000000000000000000000

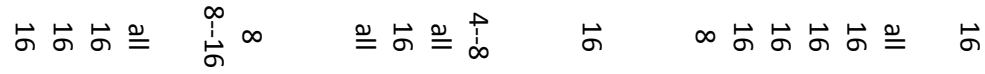

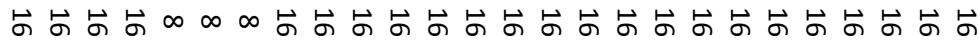

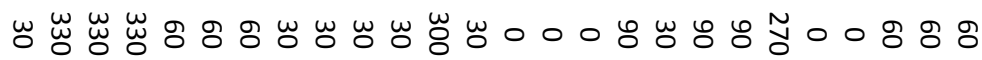

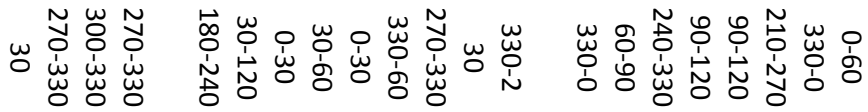




\section{Additional supplementary materials 3: Supplementary data}

S1 Data. The data presented in Fig 2.

Note:

Fig2PSTH monkeyH.txt contains the values for the population average PSTHs for monkey $\mathrm{H}$ in Figure 2A Fig2PSTH_monkeyE.txt contains the values for the population average PSTHs for monkey $\mathrm{E}$ in Figure 2B Fig2Mlvalues_monkeyH.txt contains the modulation index values for monkey $\mathrm{H}$ in Figures $2 \mathrm{C}$ and $2 \mathrm{D}$ Fig2Mlvalues_monkeyE.txt contains the modulation index values for monkey $\mathrm{E}$ in Figures $2 \mathrm{E}$ and $2 \mathrm{~F}$

Fig2PSTH_monkeyE:

\begin{tabular}{|c|c|c|c|c|c|}
\hline Time_(ms) & $P$ _attend-in & P_attend-out & AP_attend-in & $\mathrm{AP}$ _attend-ou & t Simple-saccade \\
\hline $1-2 \overline{9} 5$ & 6.8643 & 5.9907 & $\overline{6.2654}$ & $5 . \overline{9} 183$ & 6.0491 \\
\hline-285 & 6.9427 & 6.0637 & 6.1081 & 5.8055 & 6.1316 \\
\hline-275 & 6.8419 & 6.5777 & 5.9522 & 5.907 & 6.1086 \\
\hline-265 & 6.9823 & 6.7363 & 6.1045 & 5.2305 & 6.0633 \\
\hline-255 & 7.0865 & 7.047 & 6.1802 & 5.3909 & 6.5502 \\
\hline-245 & 6.8073 & 7.2828 & 5.787 & 5.3506 & 6.5601 \\
\hline-235 & 6.7682 & 7.5907 & 6.1009 & 4.6276 & 6.693 \\
\hline-225 & 7.007 & 7.8449 & 6.7315 & 4.7898 & 7.3621 \\
\hline-215 & 7.184 & 8.2418 & 6.6224 & 5.1015 & 7.4073 \\
\hline-205 & 7.4827 & 8.4272 & 6.8995 & 5.1312 & 6.9309 \\
\hline-195 & 7.926 & 8.6257 & 7.5175 & 5.2571 & 6.9887 \\
\hline-185 & 8.2704 & 8.1686 & 7.3284 & 5.8131 & 6.7593 \\
\hline-175 & 8.1043 & 7.8688 & 6.8705 & 6.1595 & 6.0916 \\
\hline-165 & 8.1921 & 7.9802 & 6.7981 & 6.3029 & 5.9605 \\
\hline-155 & 7.5422 & 8.1234 & 6.9047 & 6.016 & 5.9973 \\
\hline-145 & 7.7169 & 7.0859 & 7.1163 & 5.7055 & 6.0143 \\
\hline-135 & 7.5237 & 7.1721 & 6.6313 & 5.5733 & 6.1687 \\
\hline-125 & 7.3994 & 7.1247 & 6.3553 & 5.7005 & 6.4877 \\
\hline-115 & 6.9736 & 6.7173 & 6.1579 & 5.6512 & 6.6874 \\
\hline-105 & 7.542 & 6.5949 & 6.2025 & 6.1831 & 6.6807 \\
\hline-95 & 7.1449 & 7.4133 & 5.1948 & 6.404 & 6.5904 \\
\hline-85 & 7.1767 & 7.5083 & 5.0985 & 6.2615 & 6.5843 \\
\hline-75 & 7.1531 & 7.8896 & 5.3331 & 6.278 & 6.6385 \\
\hline-65 & 6.9939 & 7.6797 & 5.3328 & 6.1895 & 6.765 \\
\hline-55 & 6.7789 & 8.0322 & 4.4593 & 6.1144 & 7.037 \\
\hline-45 & 7.2952 & 7.807 & 4.9024 & 5.9858 & 6.6931 \\
\hline-35 & 6.5997 & 7.1869 & 4.7984 & 5.9186 & 6.6186 \\
\hline-25 & 7.1126 & 7.1784 & 5.2464 & 5.8133 & 6.6489 \\
\hline-15 & 7.8864 & 7.4067 & 6.3424 & 6.1164 & 6.572 \\
\hline-5 & 8.823 & 7.5038 & 7.4117 & 6.7671 & 6.4818 \\
\hline 31 & 13.2635 & 12.1245 & 10.3385 & 10.6774 & 6.5758 \\
\hline 15 & 24.1653 & 24.4266 & 16.5104 & 16.5346 & 7.1724 \\
\hline 25 & 38.4962 & 38.8245 & 20.3848 & 20.9588 & 7.561 \\
\hline 35 & 53.4892 & 56.3231 & 23.054 & 23.0868 & 7.9484 \\
\hline 45 & 67.9428 & 70.0988 & 23.3376 & 23.5945 & 8.3192 \\
\hline 55 & 77.4654 & 79.4004 & 21.8201 & 20.9516 & 8.6306 \\
\hline 65 & 78.9911 & 79.6599 & 17.147 & 15.9554 & 8.4085 \\
\hline 75 & 75.7192 & 74.4635 & 13.6187 & 12.2389 & 8.4724 \\
\hline 85 & 70.5429 & 66.6595 & 11.0649 & 10.1639 & 8.6221 \\
\hline 95 & 64.7104 & 60.7728 & 10.7565 & 9.8276 & 8.2949 \\
\hline 105 & 60.5882 & 54.9879 & 9.8739 & 9.1044 & 8.1156 \\
\hline 115 & 58.3844 & 50.4202 & 10.0392 & 9.1046 & 7.6174 \\
\hline 125 & 56.8897 & 48.6988 & 10.742 & 8.2565 & 7.0413 \\
\hline 135 & 56.5921 & 47.2485 & 10.1219 & 8.2507 & 6.3539 \\
\hline 145 & 56.1362 & 45.7586 & 9.9032 & 7.4872 & 5.8218 \\
\hline 155 & 54.8559 & 46.8613 & 10.1663 & 7.3394 & 5.807 \\
\hline 165 & 54.2857 & 48.4441 & 10.059 & 7.0473 & 5.8482 \\
\hline 175 & 54.3667 & 47.8982 & 8.8947 & 7.4957 & 5.5842 \\
\hline
\end{tabular}




\begin{tabular}{|c|c|c|c|c|c|c|}
\hline 49 & 185 & 53.2471 & 48.015 & 9.6651 & 7.587 & 5.5152 \\
\hline 50 & 195 & 52.8301 & 49.3076 & 9.9622 & 8.4741 & 6.0539 \\
\hline 51 & 205 & 52.6099 & 46.9615 & 9.9816 & 8.5339 & 6.32 \\
\hline 52 & 215 & 52.3183 & 43.8884 & 10.2324 & 9.3969 & 6.4275 \\
\hline 53 & 225 & 51.3302 & 44.5729 & 10.9806 & 9.2387 & 6.8207 \\
\hline 54 & 235 & 50.7501 & 42.1133 & 10.7815 & 9.8969 & 7.2764 \\
\hline 55 & 245 & 50.0488 & 40.9146 & 11.1418 & 10.2518 & 7.2709 \\
\hline 56 & 255 & 48.5066 & 41.1559 & 10.7063 & 10.6116 & 6.9037 \\
\hline 57 & 265 & 48.4299 & 41.5955 & 9.9572 & 10.1205 & 7.0101 \\
\hline 58 & 275 & 47.7662 & 40.7769 & 9.899 & 10.4532 & 6.992 \\
\hline 59 & 285 & 47.1147 & 42.2652 & 9.6176 & 9.4837 & 6.7644 \\
\hline 60 & 295 & 47.029 & 42.6726 & 9.2691 & 8.7517 & 6.8169 \\
\hline 61 & 305 & 48.9707 & 43.3173 & 10.0676 & 8.6545 & 6.8405 \\
\hline 62 & 315 & 47.8292 & 43.1471 & 10.4833 & 8.5973 & 7.1827 \\
\hline 63 & 325 & 47.4229 & 43.1757 & 11.2833 & 8.1862 & 7.12 \\
\hline 64 & 335 & 47.5508 & 41.9683 & 10.9855 & 8.7784 & 7.4115 \\
\hline 65 & 345 & 47.3137 & 40.6478 & 10.4328 & 8.3365 & 7.4647 \\
\hline 66 & 355 & 46.8646 & 39.543 & 9.5245 & 8.0955 & 8.1269 \\
\hline 67 & 365 & 47.2756 & 40.2589 & 9.4628 & 7.7351 & 7.9735 \\
\hline 68 & 375 & 47.0865 & 38.8507 & 8.6166 & 7.8718 & 7.9032 \\
\hline 69 & 385 & 47.2771 & 38.5805 & 9.0652 & 7.1582 & 7.6416 \\
\hline 70 & 395 & 46.6289 & 39.1221 & 8.6229 & 7.6873 & 7.703 \\
\hline 71 & 405 & 45.3316 & 38.9341 & 8.4959 & 7.3922 & 7.6908 \\
\hline 72 & 415 & 45.1359 & 37.4904 & 8.5845 & 7.2607 & 7.7045 \\
\hline 73 & 425 & 45.568 & 38.5132 & 8.4755 & 7.2138 & 8.0479 \\
\hline 74 & 435 & 44.8283 & 38.9639 & 8.3928 & 7.6331 & 8.1293 \\
\hline 75 & 445 & 45.2114 & 37.4274 & 8.932 & 7.1595 & 8.4795 \\
\hline 76 & 455 & 45.1588 & 36.5565 & 8.6039 & 7.5514 & 8.1139 \\
\hline 77 & 465 & 43.9859 & 36.9925 & 7.9016 & 8.4888 & 7.7816 \\
\hline 78 & 475 & 42.4697 & 35.8865 & 7.5611 & 8.8249 & 7.4721 \\
\hline 79 & 485 & 42.5969 & 35.7472 & 7.4979 & 9.1501 & 7.2867 \\
\hline 80 & 495 & 40.9023 & 37.5231 & 7.3863 & 9.341 & 6.9423 \\
\hline 81 & 505 & 41.2752 & 38.7126 & 8.392 & 8.726 & 6.6854 \\
\hline 82 & 515 & 41.2806 & 38.8393 & 8.7114 & 8.1653 & 6.8244 \\
\hline 83 & 525 & 41.9506 & 38.6504 & 8.7075 & 7.7588 & 6.98 \\
\hline 84 & 535 & 42.1627 & 39.042 & 8.7384 & 7.4309 & 6.6123 \\
\hline 85 & 545 & 43.3088 & 37.922 & 9.5248 & 7.0487 & 6.3215 \\
\hline 86 & 555 & 42.917 & 36.5581 & 9.0243 & 7.7574 & 6.0025 \\
\hline 87 & 565 & 43.9043 & 36.8331 & 9.1915 & 8.301 & 6.076 \\
\hline 88 & 575 & 44.8165 & 37.6062 & 9.0221 & 8.0828 & 5.7829 \\
\hline 89 & 585 & 44.6293 & 36.9466 & 8.9821 & 7.8369 & 5.982 \\
\hline 90 & 595 & 43.9262 & 36.7414 & 8.068 & 7.8331 & 5.796 \\
\hline
\end{tabular}

Fig2PSTH_monkeyH:

\begin{tabular}{|c|c|c|c|c|c|}
\hline Time_(ms) & $P$ _attend-in & P_attend-out & AP_attend-in & \multicolumn{2}{|c|}{ AP_attend-out Simple-saccade } \\
\hline $1-2 \overline{9} 5$ & $\overline{1} 2.1429$ & $\overline{1} 1.5615$ & 10.4756 & 12.3397 & 10.728 \\
\hline $2-285$ & 11.4081 & 11.6179 & 10.5358 & 12.7293 & 11.2092 \\
\hline-275 & 11.3363 & 11.1535 & 11.1676 & 12.24 & 11.1363 \\
\hline-265 & 10.9324 & 11.2235 & 11.0658 & 11.9961 & 11.5609 \\
\hline-255 & 10.7607 & 11.2145 & 11.082 & 12.6638 & 11.7394 \\
\hline-245 & 10.715 & 10.0289 & 12.05 & 12.6839 & 11.687 \\
\hline-235 & 11.3207 & 10.1617 & 12.8609 & 12.4806 & 11.6618 \\
\hline-225 & 10.8928 & 10.9883 & 12.5282 & 12.4542 & 12.1024 \\
\hline-215 & 10.965 & 11.4531 & 12.7668 & 13.1201 & 12.0943 \\
\hline-205 & 10.9802 & 11.5427 & 12.5275 & 12.4552 & 12.2439 \\
\hline-195 & 11.1186 & 11.9435 & 12.2353 & 12.6009 & 12.7301 \\
\hline-185 & 11.902 & 12.3394 & 12.003 & 12.8618 & 12.6851 \\
\hline-175 & 12.2422 & 12.0798 & 12.0597 & 13.0299 & 12.3712 \\
\hline-165 & 12.9166 & 11.5936 & 11.8977 & 12.808 & 12.8556 \\
\hline-155 & 12.6147 & 11.7621 & 11.9833 & 13.1112 & 13.1231 \\
\hline $16-145$ & 12.9501 & 11.6425 & 11.7107 & 12.7087 & 12.5384 \\
\hline
\end{tabular}




\begin{tabular}{|c|c|c|c|c|c|c|}
\hline 17 & -135 & 11.9641 & 11.1321 & 11.2878 & 12.5772 & 12.4268 \\
\hline 18 & -125 & 11.8054 & 12.0221 & 11.0975 & 12.0954 & 12.5258 \\
\hline 19 & -115 & 11.7338 & 11.2951 & 11.4888 & 11.5186 & 12.0528 \\
\hline 20 & -105 & 12.2882 & 10.853 & 12.0475 & 11.1021 & 12.5294 \\
\hline 21 & -95 & 12.1085 & 10.8797 & 11.9159 & 10.6637 & 11.917 \\
\hline 22 & -85 & 12.4457 & 11.1705 & 11.7246 & 10.2936 & 11.649 \\
\hline 23 & -75 & 12.4034 & 10.3263 & 11.839 & 10.7664 & 11.5439 \\
\hline 24 & -65 & 11.8128 & 10.7719 & 11.3361 & 11.3844 & 11.5576 \\
\hline 25 & -55 & 11.6828 & 10.8981 & 11.3886 & 12.1968 & 10.4235 \\
\hline 26 & -45 & 13.3484 & 12.129 & 13.2308 & 14.4701 & 11.9476 \\
\hline 27 & -35 & 14.6688 & 14.9085 & 15.7362 & 16.6901 & 14.4057 \\
\hline 28 & -25 & 17.2326 & 17.7141 & 17.9809 & 18.3258 & 16.6822 \\
\hline 29 & -15 & 19.8943 & 19.8044 & 20.1627 & 19.5797 & 18.5301 \\
\hline 30 & -5 & 21.2299 & 21.2804 & 21.5919 & 20.3215 & 20.6558 \\
\hline 31 & 5 & 23.0654 & 24.4708 & 23.1691 & 22.0593 & 19.9806 \\
\hline 32 & 15 & 31.644 & 30.8017 & 28.1785 & 26.0435 & 17.5046 \\
\hline 33 & 25 & 43.7739 & 40.8324 & 29.9506 & 28.1984 & 15.5098 \\
\hline 34 & 35 & 54.8218 & 51.2405 & 30.7758 & 29.172 & 14.4719 \\
\hline 35 & 45 & 67.7425 & 63.0609 & 32.3068 & 30.3759 & 13.1266 \\
\hline 36 & 55 & 78.3006 & 73.144 & 33.0155 & 30.4428 & 12.7034 \\
\hline 37 & 65 & 83.448 & 78.0107 & 29.5549 & 26.9793 & 12.8588 \\
\hline 38 & 75 & 84.2085 & 81.0694 & 28.0091 & 25.0639 & 11.6375 \\
\hline 39 & 85 & 86.4664 & 84.098 & 28.7557 & 24.8147 & 10.0267 \\
\hline 40 & 95 & 85.2437 & 84.2451 & 28.7931 & 25.8458 & 9.0967 \\
\hline 41 & 105 & 85.5129 & 80.9804 & 28.8485 & 24.9205 & 8.1545 \\
\hline 42 & 115 & 82.5523 & 79.6729 & 28.0116 & 24.8873 & 7.6339 \\
\hline 43 & 125 & 79.7668 & 75.3402 & 28.9601 & 26.0246 & 7.7996 \\
\hline 44 & 135 & 76.1499 & 71.2112 & 28.383 & 26.1323 & 8.4228 \\
\hline 45 & 145 & 75.0806 & 68.5873 & 27.7023 & 26.1612 & 9.0417 \\
\hline 46 & 155 & 72.8722 & 68.587 & 27.0512 & 26.2048 & 10.4076 \\
\hline 47 & 165 & 73.0604 & 65.6593 & 27.5633 & 26.3833 & 11.3047 \\
\hline 48 & 175 & 71.9665 & 63.8911 & 27.2919 & 26.2677 & 11.9577 \\
\hline 49 & 185 & 70.2321 & 62.2052 & 27.3172 & 27.0047 & 12.5888 \\
\hline 50 & 195 & 68.5066 & 59.8275 & 28.4286 & 26.5811 & 12.8145 \\
\hline 51 & 205 & 67.2255 & 57.3243 & 28.8307 & 27.0591 & 12.6929 \\
\hline 52 & 215 & 65.577 & 57.1259 & 29.4792 & 27.2909 & 12.1719 \\
\hline 53 & 225 & 64.0531 & 56.5684 & 29.9381 & 27.6181 & 12.0455 \\
\hline 54 & 235 & 63.0211 & 55.7627 & 31.4293 & 27.5571 & 11.184 \\
\hline 55 & 245 & 62.9899 & 57.5001 & 31.3329 & 27.5216 & 10.6047 \\
\hline 56 & 255 & 62.2419 & 57.764 & 30.7269 & 26.4665 & 9.4405 \\
\hline 57 & 265 & 60.2507 & 57.5727 & 29.6478 & 25.1994 & 8.999 \\
\hline 58 & 275 & 61.0187 & 58.2816 & 28.4025 & 23.7817 & 8.0719 \\
\hline 59 & 285 & 60.5248 & 58.3406 & 27.3268 & 22.3885 & 7.3311 \\
\hline 60 & 295 & 60.0672 & 57.977 & 26.4865 & 21.576 & 6.9751 \\
\hline 61 & 305 & 59.5028 & 57.7171 & 26.7879 & 20.7048 & 7.2499 \\
\hline 62 & 315 & 59.8991 & 57.0466 & 26.8603 & 21.2176 & 7.5582 \\
\hline 63 & 325 & 59.616 & 56.002 & 27.446 & 20.8891 & 8.1673 \\
\hline 64 & 335 & 60.4979 & 55.365 & 26.3832 & 20.4021 & 8.7779 \\
\hline 65 & 345 & 61.77 & 54.8385 & 26.9204 & 19.4792 & 9.2877 \\
\hline 66 & 355 & 62.5807 & 54.5521 & 25.3455 & 20.0542 & 9.9165 \\
\hline 67 & 365 & 63.7091 & 54.1162 & 24.6518 & 19.3076 & 10.3193 \\
\hline 68 & 375 & 64.2047 & 53.8163 & 24.2839 & 19.7445 & 10.3569 \\
\hline 69 & 385 & 65.2001 & 53.6106 & 23.9144 & 20.7237 & 10.5672 \\
\hline 70 & 395 & 64.4392 & 52.4517 & 22.4566 & 21.5523 & 10.6995 \\
\hline 71 & 405 & 63.5781 & 51.1137 & 23.1882 & 21.2288 & 10.9052 \\
\hline 72 & 415 & 63.0292 & 50.1711 & 23.4376 & 20.9423 & 10.811 \\
\hline 73 & 425 & 61.3393 & 49.6375 & 22.5479 & 21.0596 & 10.8136 \\
\hline 74 & 435 & 60.8051 & 49.7699 & 23.5172 & 20.3161 & 10.7349 \\
\hline 75 & 445 & 60.5636 & 49.6264 & 24.0416 & 19.5493 & 10.2185 \\
\hline 76 & 455 & 60.7205 & 50.4092 & 23.4962 & 19.4051 & 9.3384 \\
\hline 77 & 465 & 60.4047 & 50.526 & 23.5393 & 19.7046 & 8.8898 \\
\hline 78 & 475 & 60.1702 & 51.0291 & 24.367 & 18.7833 & 8.6895 \\
\hline 79 & 485 & 59.5418 & 50.8747 & 24.1428 & 19.0287 & 8.4713 \\
\hline
\end{tabular}




$\begin{array}{lllcccc}80 & 495 & 58.73 & 50.4487 & 24.5639 & 19.6488 & 8.5437 \\ 81 & 505 & 58.2412 & 50.2951 & 24.1483 & 19.9825 & 8.7452 \\ 82 & 515 & 56.3278 & 50.8432 & 23.589 & 19.9455 & 8.5183 \\ 83 & 525 & 56.1956 & 49.8552 & 23.2991 & 20.3266 & 8.332 \\ 84 & 535 & 55.2857 & 50.7582 & 22.5046 & 19.2444 & 8.4256 \\ 85 & 545 & 54.926 & 50.9781 & 22.4102 & 18.3135 & 8.5048 \\ 86 & 555 & 54.2172 & 51.3365 & 22.7945 & 18.4696 & 8.2536 \\ 87 & 565 & 56.2641 & 51.3232 & 22.9243 & 18.4819 & 8.4425 \\ 88 & 575 & 56.4323 & 51.6389 & 23.0428 & 18.5023 & 8.8724 \\ 89 & 585 & 57.5696 & 50.8237 & 23.8592 & 19.8267 & 8.6968 \\ 90 & 595 & 57.6001 & 50.318 & 22.9096 & 20.2621 & 8.263\end{array}$

Fig2Mlvalues_monkeyE:

MIPreferred_monkeyE

MIAntiPreferred_monkeyE

$\begin{array}{rll}1 & 0.20792 & 0.058824 \\ 2 & -0.038462 & -0.1039 \\ 3 & 0.027104 & 0.056122 \\ 4 & 0.055497 & 0.35714 \\ 5 & 0.14228 & 0.35 \\ 6 & 0.07907 & -0.023622 \\ 7 & -0.011765 & 0.15315 \\ 8 & 0.067692 & 0.090274 \\ 9 & 0.04142 & 0.12583 \\ 10 & 0.16753 & 0.14114 \\ 11 & 0.18798 & 0.26538 \\ 12 & 0.08169 & 0.018182 \\ 13 & 0.11193 & 0.12727 \\ 14 & 0.12 & -0.090909 \\ 15 & 0.12842 & 0.62712 \\ 16 & 0.041053 & -0.097087 \\ 17 & 0.010249 & -0.020013 \\ 18 & 0.067114 & 0.29412 \\ 19 & 0.028728 & -0.38558 \\ 20 & 0.07064 & 0.038627 \\ 21 & 0.051237 & 0.16263 \\ 22 & 0.12409 & 0.14286 \\ 23 & 0.22171 & -0.047619 \\ 24 & 0.092199 & -0.0837 \\ 25 & 0.039427 & -0.077145 \\ 26 & -0.014374 & -0.21212 \\ 27 & 0.002798 & -0.16493 \\ 28 & 0.27184 & 0.23077 \\ 29 & 0.17486 & 0.23849 \\ 30 & 0.036578 & -0.016949 \\ 31 & 0.057239 & -0.025641 \\ 32 & 0.0076336 & 0.22857 \\ 33 & 0.036739 & -0.069444 \\ 34 & 0.067215 & -0.22353 \\ 35 & 0.037736 & -0.083333 \\ 36 & 0.023041 & -0.0011751 \\ 37 & -0.19199 & -0.063415 \\ 38 & -0.043956 & 0.0013004 \\ 39 & 0.083842 & 0.056338 \\ 40 & 0.09671 & -0.18182 \\ 41 & 0.05914 & 0.095344 \\ 42 & 0.044444 & 0.21771 \\ 43 & -0.15267 & 0.28205 \\ 44 & 0.13407 & \\ & & \\ 10 & \end{array}$

Fig2Mlvalues_monkeyH:

\begin{tabular}{|c|c|c|}
\hline \multicolumn{2}{|c|}{ MIPreferred } & MIAntiPreferred \\
\hline 1 & 0.59184 & 0.11111 \\
\hline 2 & 0.0081566 & 0.40187 \\
\hline 3 & 0.036005 & 0.014925 \\
\hline 4 & 0.07109 & 0.11064 \\
\hline 5 & -0.038223 & 0.13043 \\
\hline 6 & 0.055249 & -0.015385 \\
\hline 7 & 0.023228 & 0.091703 \\
\hline 8 & 0.063142 & 0.20334 \\
\hline 9 & -0.0036765 & -0.13208 \\
\hline 10 & 0.042983 & 0.14835 \\
\hline 11 & 0.067961 & -0.058824 \\
\hline 12 & 0.0063008 & 0.0069572 \\
\hline 13 & 0.017507 & 0.26437 \\
\hline 14 & 0.11321 & 0 \\
\hline 15 & 0.077956 & 0.12444 \\
\hline 16 & -0.027027 & 0.027027 \\
\hline 17 & 0.04 & 0.02509 \\
\hline 18 & 0.1049 & -0.043643 \\
\hline 19 & -0.01002 & -0.034982 \\
\hline 20 & -0.013477 & -0.0301 \\
\hline 21 & 0.15207 & 0.21892 \\
\hline 22 & 0.074642 & 0.10386 \\
\hline 23 & 0.12814 & 0.21916 \\
\hline 24 & 0.074634 & 0.11307 \\
\hline 25 & -0.0097989 & 0.19728 \\
\hline 26 & 0.10549 & 0.013874 \\
\hline 27 & 0.023853 & -0.038407 \\
\hline 28 & -0.076923 & -0.041667 \\
\hline 29 & 0.019524 & 0.049096 \\
\hline 30 & -0.0040699 & 0.083871 \\
\hline 31 & 0.051903 & 0.10345 \\
\hline 32 & 0.0048867 & 0.015625 \\
\hline 33 & 0.036743 & -0.042821 \\
\hline 34 & 0.060878 & 0.07563 \\
\hline 35 & -0.049923 & 0.087452 \\
\hline 36 & -0.010231 & 0.19622 \\
\hline 37 & 0.045685 & 0.030303 \\
\hline 38 & -0.039834 & -0.061453 \\
\hline 39 & 0.07642 & -0.10181 \\
\hline 40 & 0.065102 & 1 \\
\hline 41 & 0.034939 & 0.062189 \\
\hline 42 & 0.06008 & 0.053892 \\
\hline 43 & 0.12886 & 0.092593 \\
\hline 44 & 0.084639 & 0.065741 \\
\hline 45 & 0.042518 & 0.027257 \\
\hline
\end{tabular}


$43 \quad 125$

$44 \quad 135$

$45 \quad 145$

$46 \quad 155$

$\begin{array}{ll}47 & 165\end{array}$

$48 \quad 175$
49

$\begin{array}{lll}49 & 185\end{array}$

$\begin{array}{ll}50 & 195\end{array}$

51205

$52 \quad 215$

$53 \quad 225$

$54 \quad 235$

$55 \quad 245$

$56 \quad 255$

$57 \quad 265$
58

$\begin{array}{ll}58 & 275\end{array}$

$59 \quad 285$

$60 \quad 295$

61305

$\begin{array}{ll}62 & 315\end{array}$

$63 \quad 325$
64

$64 \quad 335$

$65 \quad 345$

$\begin{array}{ll}66 & 355\end{array}$

$\begin{array}{ll}67 & 365\end{array}$

$\begin{array}{ll}68 & 375\end{array}$

$\begin{array}{ll}69 & 385\end{array}$

$\begin{array}{ll}70 & 395\end{array}$

$\begin{array}{ll}71 & 405\end{array}$

$\begin{array}{ll}72 & 415\end{array}$

$\begin{array}{ll}73 & 425\end{array}$

$\begin{array}{ll}74 & 435 \\ 75 & 445\end{array}$

$\begin{array}{ll}75 & 445\end{array}$

$\begin{array}{ll}76 & 455\end{array}$

$\begin{array}{ll}77 & 465\end{array}$

$\begin{array}{ll}78 & 475\end{array}$

$79 \quad 485$

$80 \quad 495$
16.5309

16.2554

16.7309

16.4872

15.9887

15.7666

15.591

15.0154

14.7174

14.8583

14.5276

14.2113

13.7895

13.4996

12.7316

12.3094

11.7077

10.9823

10.3358

10.1912

10.1259

9.8775

9.923

10.0456

10.1374

10.4151

10.7833

11.0893

11.0237

11.2345

10.9757

11.0735

11.0134

11.2289

10.7574

10.5471

9.6931

9.2936

Fig3PSTH_monkeyE:

\begin{tabular}{|c|c|c|c|}
\hline & Time_(ms) & Attend-in & Simple-saccade \\
\hline 1 & -295 & 6.6472 & 6.0491 \\
\hline 2 & -285 & 6.7594 & 6.1316 \\
\hline 3 & -275 & 6.4685 & 6.1086 \\
\hline 4 & -265 & 6.4198 & 6.0633 \\
\hline 5 & -255 & 6.4725 & 6.5502 \\
\hline & -245 & 6.6601 & 6.5601 \\
\hline 7 & -235 & 6.5333 & 6.693 \\
\hline & -225 & 6.8262 & 7.3621 \\
\hline 9 & -215 & 6.7218 & 7.4073 \\
\hline 0 & -205 & 6.5813 & 6.9309 \\
\hline 1 & -195 & 6.3631 & 6.9887 \\
\hline 2 & -185 & 6.3833 & 6.7593 \\
\hline 3 & -175 & 6.1916 & 6.0916 \\
\hline 4 & -165 & 6.2639 & 5.9605 \\
\hline 5 & -155 & 6.5886 & 5.9973 \\
\hline & -145 & 6.8516 & 6.0143 \\
\hline & -135 & 6.9291 & 6.1687 \\
\hline & -125 & 7.1972 & 6.4877 \\
\hline & -115 & 7.2494 & 6.6874 \\
\hline
\end{tabular}

7.7996

8.4228

9.0417

0.4076

11.3047

11.9577

12.5888

12.8145

2.6929

12.1719

12.0455

11.184

10.6047

. 4405

8.999

8.0719

7.3311

6.9751

7.2499

7.5582

8.1673

8.7779

9.2877

9.9165

10.3193

10.3569

10.5672

10.6995

10.9052

10.811

10.8136

10.7349

10.2185

9.3384

8.8898

8.6895

8.4713

8.5437
7.2494

6.6874

$18-125$

11.5155

125258

12.0528

12.5294

11.2955

11.917

11.649

$11.494 \quad 11.5439$

$11.752 \quad 11.5576$

$11.9717 \quad 10.4235$

$13.2051 \quad 11.9476$

$14.9251 \quad 14.4057$

$16.838 \quad 16.6822$

$18.7051 \quad 18.5301$

$19.6043 \quad 20.6558$

$18.7901 \quad 19.9806$

$17.5608 \quad 17.5046$

$16.6105 \quad 15.5098$

$15.9552 \quad 14.4719$

$15.8533 \quad 13.1266$

$16.8379 \quad 12.7034$

$17.172 \quad 12.8588$

$16.9222 \quad 11.6375$

16.8773

10.0267

9.0967

$\begin{array}{ll}16.4512 & 8.1545\end{array}$

$41 \quad 105$

7.6339 


\begin{tabular}{|c|c|c|c|}
\hline 20 & -105 & 7.1947 & 6.6807 \\
\hline 21 & -95 & 6.9072 & 6.5904 \\
\hline 22 & -85 & 7.0546 & 6.5843 \\
\hline 23 & -75 & 6.7918 & 6.6385 \\
\hline 24 & -65 & 6.7183 & 6.765 \\
\hline 25 & -55 & 6.6669 & 7.037 \\
\hline 26 & -45 & 6.5429 & 6.6931 \\
\hline 27 & -35 & 6.4175 & 6.6186 \\
\hline 28 & -25 & 6.7661 & 6.6489 \\
\hline 29 & -15 & 6.919 & 6.572 \\
\hline 30 & -5 & 6.7686 & 6.4818 \\
\hline 31 & 5 & 7.2084 & 6.5758 \\
\hline 32 & 15 & 7.7801 & 7.1724 \\
\hline 33 & 25 & 7.9965 & 7.561 \\
\hline 34 & 35 & 8.3927 & 7.9484 \\
\hline 35 & 45 & 8.8721 & 8.3192 \\
\hline 36 & 55 & 9.4038 & 8.6306 \\
\hline 37 & 65 & 9.8398 & 8.4085 \\
\hline 38 & 75 & 10.2199 & 8.4724 \\
\hline 39 & 85 & 10.5045 & 8.6221 \\
\hline 40 & 95 & 10.5824 & 8.2949 \\
\hline 41 & 105 & 9.6558 & 8.1156 \\
\hline 42 & 115 & 8.6148 & 7.6174 \\
\hline 43 & 125 & 7.7177 & 7.0413 \\
\hline 44 & 135 & 6.8957 & 6.3539 \\
\hline 45 & 145 & 6.3037 & 5.8218 \\
\hline 46 & 155 & 6.5068 & 5.807 \\
\hline 47 & 165 & 6.5004 & 5.8482 \\
\hline 48 & 175 & 6.5412 & 5.5842 \\
\hline 49 & 185 & 6.8463 & 5.5152 \\
\hline 50 & 195 & 7.4954 & 6.0539 \\
\hline 51 & 205 & 7.8047 & 6.32 \\
\hline 52 & 215 & 8.5803 & 6.4275 \\
\hline 53 & 225 & 9.0576 & 6.8207 \\
\hline 54 & 235 & 9.1675 & 7.2764 \\
\hline 55 & 245 & 9.0881 & 7.2709 \\
\hline 56 & 255 & 9.1867 & 6.9037 \\
\hline 57 & 265 & 8.8138 & 7.0101 \\
\hline 58 & 275 & 8.4911 & 6.992 \\
\hline 59 & 285 & 8.1794 & 6.7644 \\
\hline 60 & 295 & 8.0584 & 6.8169 \\
\hline 61 & 305 & 7.8894 & 6.8405 \\
\hline 62 & 315 & 8.0673 & 7.1827 \\
\hline 63 & 325 & 7.8739 & 7.12 \\
\hline 64 & 335 & 8.0086 & 7.4115 \\
\hline 65 & 345 & 8.0588 & 7.4647 \\
\hline 66 & 355 & 7.9581 & 8.1269 \\
\hline 67 & 365 & 7.516 & 7.9735 \\
\hline 68 & 375 & 7.7432 & 7.9032 \\
\hline 69 & 385 & 8.0627 & 7.6416 \\
\hline 70 & 395 & 8.1075 & 7.703 \\
\hline 71 & 405 & 8.4932 & 7.6908 \\
\hline 72 & 415 & 8.9958 & 7.7045 \\
\hline 73 & 425 & 9.2604 & 8.0479 \\
\hline 74 & 435 & 9.1299 & 8.1293 \\
\hline 75 & 445 & 9.0261 & 8.4795 \\
\hline 76 & 455 & 8.8746 & 8.1139 \\
\hline 77 & 465 & 8.2834 & 7.7816 \\
\hline 78 & 475 & 7.7849 & 7.4721 \\
\hline 79 & 485 & 7.5839 & 7.2867 \\
\hline 80 & 495 & 7.6609 & 6.9423 \\
\hline
\end{tabular}

\section{Fig3Mlvalues_monkeyH:}

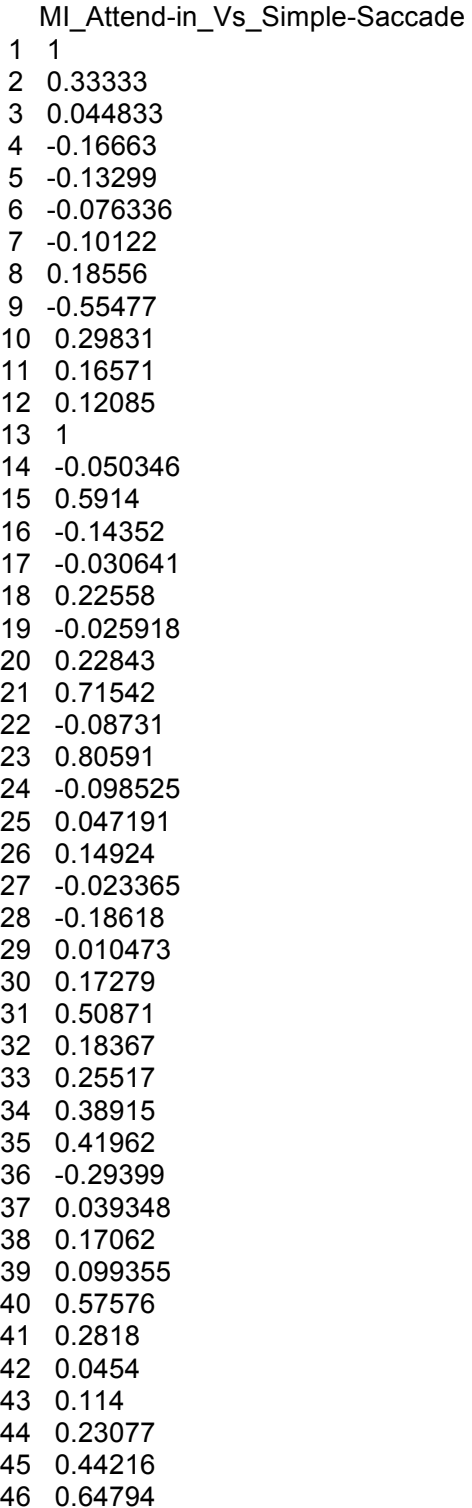

Fig3MIvalues_monkeyE:

MI Attend-in Vs Simple-Saccade
10.18455
$\begin{array}{ll}2 & 0.14851\end{array}$
$\begin{array}{lll}3 & 0.039753\end{array}$
$\begin{array}{ll}4 & -0.072411\end{array}$
$\begin{array}{ll}5 & -0.089109\end{array}$
$\begin{array}{ll}6 & 0.093473\end{array}$
$\begin{array}{lll}7 & 0.010989\end{array}$

$8 \quad 0.051456$ 

$9 \quad 0.16364$
$\begin{array}{ll}10 & -0.2053\end{array}$
$\begin{array}{ll}11 & -0.13043\end{array}$
$\begin{array}{ll}12 & 0.26381\end{array}$
$\begin{array}{ll}13 & 0.10818\end{array}$
$\begin{array}{ll}14 & -0.015773\end{array}$
$\begin{array}{ll}15 & -0.13106\end{array}$
$\begin{array}{ll}16 & 0.089813\end{array}$
$\begin{array}{ll}17 & 0.082517\end{array}$
$\begin{array}{ll}18 & 0.21053\end{array}$
$\begin{array}{ll}19 & 0.3042\end{array}$
$\begin{array}{ll}20 & 0.2587\end{array}$
$\begin{array}{ll}21 & 0.089494\end{array}$
$\begin{array}{ll}22 & -0.42717\end{array}$
$\begin{array}{ll}23 & -0.22555\end{array}$
$\begin{array}{ll}24 & 0.15843\end{array}$
$\begin{array}{ll}25 & 0.0044593\end{array}$
$26 \quad 0.012048$
$\begin{array}{ll}27 & -0.0074231\end{array}$
$28 \quad 0.28405$
$\begin{array}{ll}29 & 0.13287\end{array}$
$\begin{array}{ll}30 & -0.05618\end{array}$
$\begin{array}{ll}31 & 0.14591\end{array}$
$\begin{array}{ll}32 & 0.04451\end{array}$
$\begin{array}{ll}33 & -0.0016849\end{array}$
$\begin{array}{ll}34 & 0.071262\end{array}$
$\begin{array}{ll}35 & 0.14897\end{array}$
$\begin{array}{ll}36 & 0.24409\end{array}$
$\begin{array}{ll}37 & -0.035294\end{array}$
$\begin{array}{ll}38 & 0.083478\end{array}$
$39 \quad 0.15111$
$\begin{array}{ll}40 & 0.13852\end{array}$
$\begin{array}{ll}41 & 0.027027\end{array}$
$\begin{array}{ll}42 & 0.047486\end{array}$
$\begin{array}{ll}43 & -0.49677\end{array}$
$\begin{array}{ll}44 & -0.53982\end{array}$ 


\section{S3 Data. The data presented in Fig 4.}

Note:

Fig4PSTH_monkeyH.txt contains the values for the population average PSTHs for monkey $\mathrm{H}$ in Figure 4A Fig4PSTH_monkeyE.txt contains the values for the population average PSTHs for monkey E in Figure 4B Fig4Mlvalues_monkeyH.txt contains the modulation index values for monkey $\mathrm{H}$ in Figures 4C,D Fig4MIvalues_monkeyE.txt contains the modulation index values for monkey $\mathrm{E}$ in Figures 4E,F

Fig4PSTH_monkeyH:

\begin{tabular}{|c|c|c|c|c|}
\hline & Time_(ms) & Attend-in & Attend-out & Simple-saccade \\
\hline 1 & -295 & 11.4518 & 11.9827 & 10.728 \\
\hline 2 & -285 & 11.4876 & 11.7978 & 11.2092 \\
\hline 3 & -275 & 11.4812 & 11.7765 & 11.1363 \\
\hline 4 & -265 & 11.4137 & 11.5042 & 11.5609 \\
\hline 5 & -255 & 11.2676 & 11.7192 & 11.7394 \\
\hline 6 & -245 & 11.5908 & 11.4684 & 11.687 \\
\hline 7 & -235 & 11.7919 & 11.9624 & 11.6618 \\
\hline 8 & -225 & 11.9095 & 12.4152 & 12.1024 \\
\hline 9 & -215 & 12.0714 & 12.8407 & 12.0943 \\
\hline 10 & -205 & 12.4413 & 12.76 & 12.2439 \\
\hline 11 & -195 & 12.5062 & 12.7933 & 12.7301 \\
\hline 12 & -185 & 11.9943 & 12.6312 & 12.6851 \\
\hline 13 & -175 & 11.8636 & 12.4748 & 12.3712 \\
\hline 14 & -165 & 11.6359 & 12.415 & 12.8556 \\
\hline 15 & -155 & 11.3872 & 12.1764 & 13.1231 \\
\hline 16 & -145 & 11.234 & 12.2154 & 12.5384 \\
\hline 17 & -135 & 11.5155 & 12.1638 & 12.4268 \\
\hline 18 & -125 & 11.4528 & 12.3346 & 12.5258 \\
\hline 19 & -115 & 11.2976 & 12.3557 & 12.0528 \\
\hline 20 & -105 & 11.3073 & 12.7254 & 12.5294 \\
\hline 21 & -95 & 11.2955 & 12.6463 & 11.917 \\
\hline 22 & -85 & 11.3825 & 12.6727 & 11.649 \\
\hline 23 & -75 & 11.494 & 12.6505 & 11.5439 \\
\hline 24 & -65 & 11.752 & 12.3168 & 11.5576 \\
\hline 25 & -55 & 11.9717 & 12.3074 & 10.4235 \\
\hline 26 & -45 & 13.2051 & 13.6185 & 11.9476 \\
\hline 27 & -35 & 14.9251 & 15.7281 & 14.4057 \\
\hline 28 & -25 & 16.838 & 17.1761 & 16.6822 \\
\hline 29 & -15 & 18.7051 & 18.7173 & 18.5301 \\
\hline 30 & -5 & 19.6043 & 19.962 & 20.6558 \\
\hline 31 & 5 & 18.7901 & 19.0231 & 19.9806 \\
\hline 32 & 15 & 17.5608 & 17.1053 & 17.5046 \\
\hline 33 & 25 & 16.6105 & 16.2872 & 15.5098 \\
\hline 34 & 35 & 15.9552 & 15.9863 & 14.4719 \\
\hline 35 & 45 & 15.8533 & 15.0264 & 13.1266 \\
\hline 36 & 55 & 16.8379 & 14.9154 & 12.7034 \\
\hline 37 & 65 & 17.172 & 14.6465 & 12.8588 \\
\hline 38 & 75 & 16.9222 & 13.6597 & 11.6375 \\
\hline 39 & 85 & 16.8773 & 12.3011 & 10.0267 \\
\hline 40 & 95 & 16.7612 & 11.6608 & 9.0967 \\
\hline 41 & 105 & 16.4512 & 11.0319 & 8.1545 \\
\hline 42 & 115 & 16.4903 & 10.7706 & 7.6339 \\
\hline 43 & 125 & 16.5309 & 10.9795 & 7.7996 \\
\hline 44 & 135 & 16.2554 & 11.2042 & 8.4228 \\
\hline 45 & 145 & 16.7309 & 11.0155 & 9.0417 \\
\hline 46 & 155 & 16.4872 & 11.4639 & 10.4076 \\
\hline 47 & 165 & 15.9887 & 11.832 & 11.3047 \\
\hline 48 & 175 & 15.7666 & 12.0892 & 11.9577 \\
\hline 49 & 185 & 15.591 & 12.0405 & 12.5888 \\
\hline 50 & 195 & 15.0154 & 12.4861 & 12.8145 \\
\hline
\end{tabular}




$\begin{array}{lllll}51 & 205 & 14.7174 & 13.171 & 12.6929 \\ 52 & 215 & 14.8583 & 13.0928 & 12.1719 \\ 53 & 225 & 14.5276 & 12.7933 & 12.0455 \\ 54 & 235 & 14.2113 & 12.7198 & 11.184 \\ 55 & 245 & 13.7895 & 12.3831 & 10.6047 \\ 56 & 255 & 13.4996 & 11.2171 & 9.4405 \\ 57 & 265 & 12.7316 & 10.6032 & 8.999 \\ 58 & 275 & 12.3094 & 10.2022 & 8.0719 \\ 59 & 285 & 11.7077 & 9.9963 & 7.3311 \\ 60 & 295 & 10.9823 & 9.3598 & 6.9751 \\ 61 & 305 & 10.3358 & 9.026 & 7.2499 \\ 62 & 315 & 10.1912 & 8.681 & 7.5582 \\ 63 & 325 & 10.1259 & 8.4872 & 8.1673 \\ 64 & 335 & 9.8775 & 8.1278 & 8.7779 \\ 65 & 345 & 9.923 & 8.2983 & 9.2877 \\ 66 & 355 & 10.0456 & 8.4438 & 9.9165 \\ 67 & 365 & 10.1374 & 8.8727 & 10.3193 \\ 68 & 375 & 10.4151 & 9.1382 & 10.3569 \\ 69 & 385 & 10.7833 & 9.5917 & 10.5672 \\ 70 & 395 & 11.0893 & 9.8954 & 10.6995 \\ 71 & 405 & 11.0237 & 10.2827 & 10.9052 \\ 72 & 415 & 11.2345 & 10.3578 & 10.811 \\ 73 & 425 & 10.9757 & 10.1934 & 10.8136 \\ 74 & 435 & 11.0735 & 10.1678 & 10.7349 \\ 75 & 445 & 11.0134 & 10.2026 & 10.2185 \\ 76 & 455 & 11.2289 & 10.4017 & 9.3384 \\ 77 & 465 & 10.7574 & 10.3452 & 8.8898 \\ 78 & 475 & 10.5471 & 10.2031 & 8.6895 \\ 79 & 485 & 9.6931 & 9.616 & 8.4713 \\ 80 & 495 & 9.2936 & 8.9847 & 8.5437\end{array}$

Fig4PSTH_monkeyE:

\begin{tabular}{|c|c|c|c|c|}
\hline & Time_(ms) & Attend-in & Attend-out & Simple-saccade \\
\hline 1 & -295 & 6.6472 & 6.6631 & 6.0491 \\
\hline 2 & -285 & 6.7594 & 6.5231 & 6.1316 \\
\hline 3 & -275 & 6.4685 & 6.6156 & 6.1086 \\
\hline 4 & -265 & 6.4198 & 6.642 & 6.0633 \\
\hline 5 & -255 & 6.4725 & 6.6274 & 6.5502 \\
\hline 6 & -245 & 6.6601 & 6.7036 & 6.5601 \\
\hline 7 & -235 & 6.5333 & 6.8117 & 6.693 \\
\hline 8 & -225 & 6.8262 & 6.9909 & 7.3621 \\
\hline 9 & -215 & 6.7218 & 6.8842 & 7.4073 \\
\hline 10 & -205 & 6.5813 & 6.8382 & 6.9309 \\
\hline 11 & -195 & 6.3631 & 6.686 & 6.9887 \\
\hline 12 & -185 & 6.3833 & 6.6863 & 6.7593 \\
\hline 13 & -175 & 6.1916 & 6.6801 & 6.0916 \\
\hline 14 & -165 & 6.2639 & 6.6356 & 5.9605 \\
\hline 15 & -155 & 6.5886 & 6.7658 & 5.9973 \\
\hline 16 & -145 & 6.8516 & 6.8396 & 6.0143 \\
\hline 17 & -135 & 6.9291 & 6.5372 & 6.1687 \\
\hline 18 & -125 & 7.1972 & 6.334 & 6.4877 \\
\hline 19 & -115 & 7.2494 & 6.4056 & 6.6874 \\
\hline 20 & -105 & 7.1947 & 6.2785 & 6.6807 \\
\hline 21 & -95 & 6.9072 & 6.2594 & 6.5904 \\
\hline 22 & -85 & 7.0546 & 6.5267 & 6.5843 \\
\hline 23 & -75 & 6.7918 & 6.6197 & 6.6385 \\
\hline 24 & -65 & 6.7183 & 6.8254 & 6.765 \\
\hline 25 & -55 & 6.6669 & 7.0085 & 7.037 \\
\hline 26 & -45 & 6.5429 & 6.6829 & 6.6931 \\
\hline 27 & -35 & 6.4175 & 6.5372 & 6.6186 \\
\hline 28 & -25 & 6.7661 & 6.7878 & 6.6489 \\
\hline 29 & -15 & 6.919 & 6.734 & 6.572 \\
\hline
\end{tabular}




$\begin{array}{lllll}30 & -5 & 6.7686 & 6.6326 & 6.4818 \\ 31 & 5 & 7.2084 & 7.0821 & 6.5758 \\ 32 & 15 & 7.7801 & 7.7777 & 7.1724 \\ 33 & 25 & 7.9965 & 7.9323 & 7.561 \\ 34 & 35 & 8.3927 & 8.3153 & 7.9484 \\ 35 & 45 & 8.8721 & 8.8874 & 8.3192 \\ 36 & 55 & 9.4038 & 9.095 & 8.6306 \\ 37 & 65 & 9.8398 & 9.2681 & 8.4085 \\ 38 & 75 & 10.2199 & 9.6345 & 8.4724 \\ 39 & 85 & 10.5045 & 9.896 & 8.6221 \\ 40 & 95 & 10.5824 & 9.6585 & 8.2949 \\ 41 & 105 & 9.6558 & 9.1812 & 8.1156 \\ 42 & 115 & 8.6148 & 8.2455 & 7.6174 \\ 43 & 125 & 7.7177 & 7.5903 & 7.0413 \\ 44 & 135 & 6.8957 & 6.6869 & 6.3539 \\ 45 & 145 & 6.3037 & 6.216 & 5.8218 \\ 46 & 155 & 6.5068 & 6.0254 & 5.807 \\ 47 & 165 & 6.5004 & 6.0295 & 5.8482 \\ 48 & 175 & 6.5412 & 5.9211 & 5.5842 \\ 49 & 185 & 6.8463 & 6.2256 & 5.5152 \\ 50 & 195 & 7.4954 & 6.4991 & 6.0539 \\ 51 & 205 & 7.8047 & 6.9085 & 6.32 \\ 52 & 215 & 8.5803 & 7.5514 & 6.4275 \\ 53 & 225 & 9.0576 & 7.8899 & 6.8207 \\ 54 & 235 & 9.1675 & 8.0987 & 7.2764 \\ 55 & 245 & 9.0881 & 8.4481 & 7.2709 \\ 56 & 255 & 9.1867 & 8.6861 & 6.9037 \\ 57 & 265 & 8.8138 & 8.7158 & 7.0101 \\ 58 & 275 & 8.4911 & 8.6336 & 6.992 \\ 59 & 285 & 8.1794 & 8.3631 & 6.7644 \\ 60 & 295 & 8.0584 & 8.15 & 6.8169 \\ 61 & 305 & 7.8894 & 7.8575 & 6.8405 \\ 62 & 315 & 8.0673 & 7.5547 & 7.1827 \\ 63 & 325 & 7.8739 & 7.6964 & 7.12 \\ 64 & 335 & 8.0086 & 7.9964 & 7.4115 \\ 65 & 345 & 8.0588 & 8.0935 & 7.4647 \\ 66 & 355 & 7.9581 & 8.2146 & 8.1269 \\ 67 & 365 & 7.516 & 8.3196 & 7.9735 \\ 68 & 375 & 7.7432 & 8.0215 & 7.9032 \\ 69 & 385 & 8.0627 & 8.0136 & 7.6416 \\ 70 & 395 & 8.1075 & 8.0841 & 7.703 \\ 71 & 405 & 8.4932 & 8.392 & 7.6908 \\ 72 & 415 & 8.9958 & 8.7001 & 7.7045 \\ 73 & 425 & 9.2604 & 9.031 & 8.0479 \\ 74 & 435 & 9.1299 & 9.0852 & 8.1293 \\ 75 & 445 & 9.0261 & 8.9357 & 8.4795 \\ 76 & 455 & 8.8746 & 8.7573 & 8.1139 \\ 77 & 465 & 8.2834 & 8.2551 & 7.7816 \\ 78 & 475 & 7.7849 & 8.4226 & 7.4721 \\ 79 & 485 & 7.5839 & 8.3619 & 7.2867 \\ 80 & 495 & 7.6609 & 8.3591 & 6.9423\end{array}$


Fig4MIvalues_monkeyH:

MI_Attend-in_Vs_Attend-out out_Vs_Simple-Saccade $10.6 \overline{4} 858$

20.049668

$3 \quad 0.18042$

40.11086

$5-0.02605$

$6-0.051765$

$7 \quad-7.2438 e-05$

80.036436

$\begin{array}{ll}9 & -0.25\end{array}$

$10 \quad 0.088058$

110.16239

120.040986

130

140.3942

$\begin{array}{ll}15 & 0.51899\end{array}$

$16 \quad 0.17005$

$17-0.055375$

180.041609

$19 \quad 0.28172$

$20 \quad 0.15001$

210.64636

$22-0.16638$

$23 \quad 0.56575$

$24-0.033969$

$25 \quad 0.12301$

$26 \quad 0.14656$

$27 \quad 0.028424$

$28-0.16832$

$\begin{array}{ll}29 & 0.12583\end{array}$

$30 \quad 0.026942$

310.30901

$32 \quad 0.27921$

330.066955

$34 \quad 0.22856$

$35 \quad 0.10811$

$36-0.026142$

370.0023145

$38 \quad 0.16773$

$\begin{array}{ll}39 & 0.12947\end{array}$

$40 \quad 0.17785$

$\begin{array}{ll}41 & 0.092154\end{array}$

420.11465

$43 \quad 0.038419$

$\begin{array}{ll}44 & 0.11669\end{array}$

$\begin{array}{ll}45 & 0.21672\end{array}$

$\begin{array}{ll}46 & 0.66038\end{array}$

Fig4MIvalues_monkeyE:

MI Attend-in Vs Attend-out out_Vs_Simple-Saccade

$1 \overline{0} .104$

20.067485

$3-0.029204$

40.12821

$\begin{array}{ll}5 & 0.125\end{array}$

$\begin{array}{ll}6 & 0.063063\end{array}$

7 8.3267e-17
MI_Attend-

0.082126

0.081851

0.068878

$-0.19877$

$-0.21175$

0.03059

MI Attend-

0.28844

$-0.1367$

$-0.10731$

$-0.024668$

$-0.10115$

$-0.35385$

0.21592

$-0.4359$

0.10448

0.024776

0.18571

0.081197

0.12846

0.080238

$-0.064773$

$-0.076264$

- n t

$-0.051754$

$-0.11551$

0.14653

.23695

0.19149

0.17628

0.32632

0.0029674

$-0.030508$

0.1947

$-0.069612$

0.11723

0.24933

$-0.021739$

0.010989

$\begin{array}{clc}8 & 0.064102 & -0.012688 \\ 9 & 0.20962 & -0.047619 \\ 10 & -0.12145 & -0.085989 \\ 11 & -0.28926 & 0.16505 \\ 12 & 0.14258 & 0.12597 \\ 13 & -0.011537 & 0.11957 \\ 14 & 0.3 & -0.31429 \\ 15 & 0.29412 & -0.4094 \\ 16 & -0.028405 & 0.11792 \\ 17 & -0.077294 & 0.1588 \\ 18 & 0.044927 & 0.16718 \\ 19 & 0.012048 & 0.29322 \\ 20 & 0.31646 & -0.062907 \\ 21 & 0.033058 & 0.056604 \\ 22 & -0.34177 & -0.1 \\ 23 & 0.14176 & -0.35593 \\ 24 & -0.042394 & 0.19949 \\ 25 & -0.022291 & 0.026747 \\ 26 & 0.16584 & -0.1541 \\ 27 & -0.024619 & 0.017199 \\ 28 & 0.11864 & 0.17117 \\ 29 & 0.24276 & -0.11356 \\ 30 & 0.081397 & -0.13695 \\ 31 & 0.13078 & 0.015431 \\ 32 & -0.00094607 & 0.045455 \\ 33 & -0.10848 & 0.10682 \\ 34 & 0.13728 & -0.066667 \\ 35 & 0.14897 & 0 \\ 36 & 0.032393 & 0.21339 \\ 37 & 0.09024 & -0.12514 \\ 38 & 0.058872 & 0.024728 \\ 39 & 0.30997 & -0.16667 \\ 40 & 0.067354 & 0.071838 \\ 41 & 0.16014 & -0.13369 \\ 42 & 0.21176 & -0.16594 \\ 43 & 0.016949 & -0.39535 \\ 44 & -0.18367 & \end{array}$

S4 Data. The data presented in Fig 5:

Note:

Fig5DIvalues_monkeyH.txt contains the direction-tuning index values for monkey $\mathrm{H}$ in Figures $5 \mathrm{~A}$ and $5 \mathrm{~B}$

Fig5DIvalues_monkeyE.txt contains the directiontuning index values for monkey $\mathrm{E}$ in Figures $5 \mathrm{C}$ and 5D

HeldOutTrials_DIvalues_monkeyH.txt contains the direction-tuning index values for monkey $\mathrm{H}$ reported in the text using only the held-out trials HeldOutTrials DIvalues monkeyE.txt contains the direction-tuning index values for monkey $\mathrm{H}$ reported in the text using only the held-out trials

Fig5Dlvalues_monkeyH:

DIAttendln_PvsAP DIAttendOut_PvsAP
$1-0.40659$
0.034483
20.15789
0.1276
$3-0.067521$
$-0.13559$
$4-0.17726$
$-0.26154$ 


$\begin{array}{clc}5 & 0.028902 & 0.0097561 \\ 6 & -0.01179 & 0.064962 \\ 7 & -0.025086 & 0.019685 \\ 8 & -1.1211 \mathrm{e}-16 & 0.081413 \\ 9 & 0.33333 & -0.62832 \\ 10 & 0.099778 & -8.2239 \mathrm{e}-17 \\ 11 & 0.041667 & -0.048456 \\ 12 & -0.00051467 & -0.031752 \\ 13 & -0.26316 & -0.11325 \\ 14 & 0.38462 & 0.11765 \\ 15 & -0.0625 & -0.013333 \\ 16 & 0.15 & -0.084681 \\ 17 & 0.046729 & 0.03936 \\ 18 & 0.10128 & 0.088353 \\ 19 & -0.02439 & 0.053763 \\ 20 & 0.018182 & 0.042025 \\ 21 & -0.0045366 & 0.11374 \\ 22 & -0.064669 & 0.14132 \\ 23 & 0.069909 & 0.013398 \\ 24 & 0.19192 & -0.18495 \\ 25 & -0.012876 & -0.034 \\ 26 & 0.093525 & 0.093539 \\ 27 & -0.070352 & 0.010638 \\ 28 & 0 & 0.084746 \\ 29 & 0.035294 & 0.080808 \\ 30 & 0.028953 & -0.013453 \\ 31 & 0.088172 & -0.038082 \\ 32 & 0.017241 & 0.104 \\ 33 & -0.00061237 & 0.11504 \\ 34 & 0.069359 & 0.16176 \\ 35 & 0.02439 & 0.090909 \\ 36 & 0.096774 & 0.14754 \\ 37 & 0.034655 & 0.0054945 \\ 38 & -0.11864 & 0.03537 \\ 39 & 0.017594 & -0.047715 \\ 40 & -0.15385 & 0.14286 \\ 41 & 0.097276 & 0.058252 \\ 42 & -0.1011 & -0.10852 \\ 43 & -0.034301 & 0.19325 \\ 44 & 0.049236 & -0.067894 \\ 45 & 0.073684 & 0.036429 \\ 46 & -0.11542 & -0.13208 \\ & & \end{array}$

Fig5Dlvalues_monkeyE:

\begin{tabular}{|c|c|c|}
\hline & \multicolumn{2}{|c|}{ DIAttendIn_PvsAP } \\
\hline 1 & 0.059289 & -0.034965 \\
\hline 2 & -0.12853 & -0.04451 \\
\hline 3 & -0.040971 & -0.047819 \\
\hline 4 & -0.16024 & 0.35484 \\
\hline 5 & -0.54 & -0.066667 \\
\hline 6 & 0.018834 & -0.020576 \\
\hline 7 & -0.073171 & -0.16088 \\
\hline 8 & 0.033708 & -0.038298 \\
\hline & -0.043478 & -0.6 \\
\hline & -0.28302 & -0.28467 \\
\hline & -0.025641 & -0.67939 \\
\hline & -0.39433 & -0.011765 \\
\hline & -0.041913 & 0.066128 \\
\hline & -0.18919 & -0.39241 \\
\hline & -0.20988 & -0.31343 \\
\hline & 0.1209 & 0.09589 \\
\hline
\end{tabular}

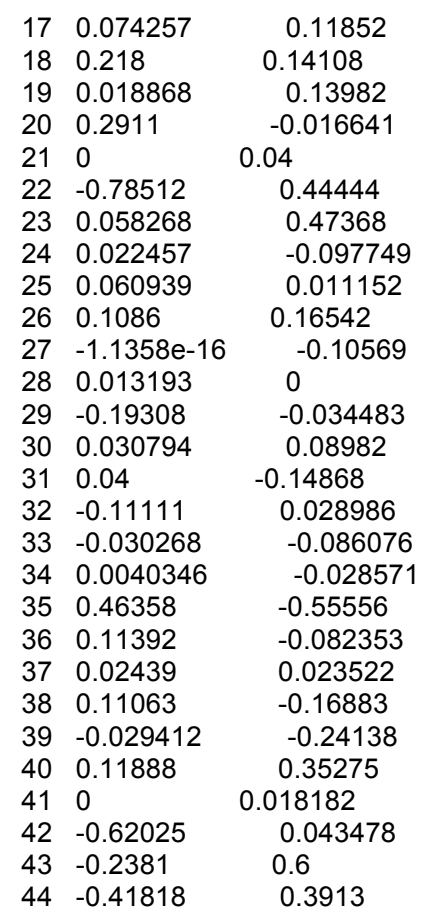

HeldOutTrials_Dlvalues_monkeyH:

\begin{tabular}{|c|c|c|}
\hline \multicolumn{2}{|c|}{ DIAttendIn_PvsAP } & DIAttendOut_PvsAP \\
\hline 1 & $-0.68421-$ & - \\
\hline 2 & 0.17757 & 0.090909 \\
\hline 3 & 0.087799 & 0.24611 \\
\hline 4 & 0.17391 & 0.37143 \\
\hline 5 & -0.10414 & -0.042017 \\
\hline 6 & -0.069054 & 0.081511 \\
\hline 7 & -0.04644 & -0.012061 \\
\hline 8 & -0.14146 & 0.11628 \\
\hline 9 & 1 & 1 \\
\hline 10 & -0.10942 & -0.2691 \\
\hline 11 & -0.018182 & 0.013699 \\
\hline 12 & -0.017544 & -0.022288 \\
\hline 13 & 0.41176 & 0.029412 \\
\hline 14 & 0.51899 & -0.093525 \\
\hline 15 & -1 & -0.17647 \\
\hline 16 & 0.052632 & 0.16667 \\
\hline 17 & -0.093562 & -0.11558 \\
\hline 18 & 0.0027248 & -0.14826 \\
\hline 19 & -0.5 & -0.044177 \\
\hline 20 & -0.10828 & -0.093333 \\
\hline 21 & -0.13043 & -0.30165 \\
\hline 22 & -0.17073 & 0.016949 \\
\hline 23 & 0.10638 & -0.12821 \\
\hline 24 & 0.30769 & 0.29412 \\
\hline 25 & -0.03949 & -0.13995 \\
\hline 26 & 0.083333 & -0.017341 \\
\hline 27 & -0.18462 & -0.088083 \\
\hline 28 & -0.072797 & 0.07563 \\
\hline 29 & 0.045783 & -0.017964 \\
\hline 30 & -0.0090439 & 0.003337 \\
\hline
\end{tabular}




$\begin{array}{llc}31 & 0.031161 & 0.056604 \\ 32 & 0.0015504 & 0.02439 \\ 33 & -0.022556 & 0.1954 \\ 34 & 0.125 & 0.20313 \\ 35 & -0.33023 & -0.21212 \\ 36 & -0.12108 & 0.098592 \\ 37 & 0.058824 & -0.057778 \\ 38 & -0.12 & -0.13514 \\ 39 & -0.08835 & 0.074024 \\ 40 & 0.061453 & 0.17419 \\ 41 & -0.031746 & 0.098039 \\ 42 & 0.032617 & 0.069807 \\ 43 & -0.0082919 & -0.074074 \\ 44 & 0.077966 & -0.14953 \\ 45 & 0.093946 & 0.02958 \\ 46 & 0.18519 & 0.076923\end{array}$

HeldOutTrials_Dlvalues_monkeyE:

\begin{tabular}{|c|c|c|}
\hline \multicolumn{3}{|c|}{ DIAttendln_PvsAP } \\
\hline 1 & -0.133 & 0.028002 \\
\hline 2 & 0.10141 & 0.062295 \\
\hline 3 & 0.027523 & 0.05174 \\
\hline 4 & -0.35484 & -0.1 \\
\hline 5 & 0.53125 & -0.29412 \\
\hline 6 & -0.02439 & -0.12621 \\
\hline 7 & -0.11765 & 0.075795 \\
\hline 8 & 0.034091 & 0.0079194 \\
\hline 9 & -0.043478 & 0.2973 \\
\hline 10 & 0.052632 & -0.8 \\
\hline 11 & -1 & -1 \\
\hline 12 & 0.31646 & -0.089457 \\
\hline 13 & -0.18143 & 0.060847 \\
\hline 14 & 0.28571 & 0.41176 \\
\hline 15 & 0.33333 & 0.41176 \\
\hline 16 & 0.22849 & 0.086614 \\
\hline 17 & 0.09589 & 0.078498 \\
\hline 18 & 0.10569 & 0.1236 \\
\hline 19 & -0.11789 & 0.24068 \\
\hline 20 & 0.75 & -0.1588 \\
\hline 21 & -1 & -0.86667 \\
\hline 22 & 1 & 0.74257 \\
\hline 23 & -0.066667 & 0.55556 \\
\hline 24 & 0.017544 & 0.12088 \\
\hline 25 & 0.023256 & -0.004329 \\
\hline 26 & 0.22222 & 0.61062 \\
\hline 27 & -0.09589 & 0.066667 \\
\hline 28 & -0.015625 & -0.46667 \\
\hline 29 & 0.2844 & -0.058824 \\
\hline 30 & 0.012412 & 0.2 \\
\hline 31 & -0.10698 & 0.21053 \\
\hline 32 & 0.072423 & -0.14655 \\
\hline 33 & 0.050847 & -0.026946 \\
\hline 34 & $6.2172 \mathrm{e}-17$ & -0.13604 \\
\hline 35 & 0.48148 & 0.42675 \\
\hline 36 & 0.086876 & 0.018568 \\
\hline 37 & 0.018739 & -0.16129 \\
\hline 38 & -0.38776 & -0.33119 \\
\hline 39 & -0.090909 & 0 \\
\hline 40 & 0.16667 & 0.22222 \\
\hline 41 & -0.2 & -0.04 \\
\hline 42 & 0.65829 & -0.47826 \\
\hline
\end{tabular}
$43 \quad 0.33333$
441
0.45946
0.56522

\section{S5 Data. The data presented in Fig 6}

Note:

Each file contains the data for the PSTHs (A-D) and mean difference / confidence-bands (E-H).

\section{Fig6APSTH_monkeyH:}

\begin{tabular}{|c|c|c|c|}
\hline & Time_(ms) & Preferred & Anti-preferred \\
\hline 1 & -295 & 10.8977 & 12.089 \\
\hline 2 & -285 & 11.1259 & 11.8708 \\
\hline 3 & -275 & 11.3766 & 11.5292 \\
\hline 4 & -265 & 11.3496 & 11.3966 \\
\hline 5 & -255 & 11.5501 & 10.8345 \\
\hline 6 & -245 & 12.1066 & 10.9444 \\
\hline 7 & -235 & 12.1778 & 11.2943 \\
\hline 8 & -225 & 12.3774 & 11.4546 \\
\hline 9 & -215 & 12.2332 & 11.9179 \\
\hline 10 & -205 & 12.2384 & 12.7147 \\
\hline 11 & -195 & 12.1064 & 12.9404 \\
\hline 12 & -185 & 11.5609 & 12.5034 \\
\hline 13 & -175 & 10.9057 & 12.8654 \\
\hline 14 & -165 & 11.2328 & 12.2025 \\
\hline 15 & -155 & 11.1138 & 11.8228 \\
\hline 16 & -145 & 10.8973 & 11.7071 \\
\hline 17 & -135 & 11.4664 & 11.6502 \\
\hline 18 & -125 & 11.8695 & 11.0707 \\
\hline 19 & -115 & 11.6766 & 10.8474 \\
\hline 20 & -105 & 11.6553 & 10.9077 \\
\hline 21 & -95 & 11.5841 & 11.0119 \\
\hline 22 & -85 & 11.1708 & 11.535 \\
\hline 23 & -75 & 11.0086 & 11.919 \\
\hline 24 & -65 & 10.895 & 12.5199 \\
\hline 25 & -55 & 11.1046 & 12.6804 \\
\hline 26 & -45 & 12.2192 & 14.061 \\
\hline 27 & -35 & 14.1743 & 15.7038 \\
\hline 28 & -25 & 16.1167 & 17.5641 \\
\hline 29 & -15 & 18.3768 & 19.0211 \\
\hline 30 & -5 & 19.5915 & 19.6299 \\
\hline 31 & 5 & 19.1716 & 18.3791 \\
\hline 32 & 15 & 17.9945 & 16.9875 \\
\hline 33 & 25 & 17.3827 & 15.7553 \\
\hline 34 & 35 & 16.5884 & 15.2491 \\
\hline 35 & 45 & 15.9411 & 15.647 \\
\hline 36 & 55 & 17.0018 & 16.5699 \\
\hline 37 & 65 & 17.3752 & 16.9114 \\
\hline 38 & 75 & 16.9936 & 16.7714 \\
\hline 39 & 85 & 16.8463 & 16.8399 \\
\hline 40 & 95 & 17.0383 & 16.4564 \\
\hline 41 & 105 & 16.4595 & 16.4402 \\
\hline 42 & 115 & 16.535 & 16.3817 \\
\hline 43 & 125 & 16.4125 & 16.5911 \\
\hline 44 & 135 & 16.4793 & 15.9825 \\
\hline 45 & 145 & 16.6256 & 16.8542 \\
\hline 46 & 155 & 16.3114 & 16.709 \\
\hline 47 & 165 & 16.039 & 16.0221 \\
\hline 48 & 175 & 15.9572 & 15.6226 \\
\hline 49 & 185 & 15.4841 & 15.7455 \\
\hline 50 & 195 & 14.9995 & 14.9583 \\
\hline
\end{tabular}




$\begin{array}{llll}51 & 205 & 15.1169 & 14.1862 \\ 52 & 215 & 15.2321 & 14.3117 \\ 53 & 225 & 15.2535 & 13.695 \\ 54 & 235 & 15.0617 & 13.2541 \\ 55 & 245 & 14.9217 & 12.5911 \\ 56 & 255 & 14.348 & 12.5925 \\ 57 & 265 & 13.048 & 12.3824 \\ 58 & 275 & 12.2545 & 12.3342 \\ 59 & 285 & 11.6941 & 11.748 \\ 60 & 295 & 11.0258 & 10.9852 \\ 61 & 305 & 10.6543 & 10.0807 \\ 62 & 315 & 10.8738 & 9.5561 \\ 63 & 325 & 10.9338 & 9.3545 \\ 64 & 335 & 10.733 & 9.0021 \\ 65 & 345 & 10.5609 & 9.2453 \\ 66 & 355 & 10.4257 & 9.5446 \\ 67 & 365 & 10.7225 & 9.4662 \\ 68 & 375 & 10.9237 & 9.7891 \\ 69 & 385 & 10.9506 & 10.4914 \\ 70 & 395 & 11.2306 & 10.8319 \\ 71 & 405 & 11.0122 & 10.9832 \\ 72 & 415 & 11.162 & 11.2434 \\ 73 & 425 & 10.7833 & 11.1245 \\ 74 & 435 & 11.0825 & 11.0397 \\ 75 & 445 & 11.0875 & 10.894 \\ 76 & 455 & 11.4429 & 10.995 \\ 77 & 465 & 10.6452 & 10.9015 \\ 78 & 475 & 10.4559 & 10.6517 \\ 79 & 485 & 9.6027 & 9.8106 \\ 80 & 495 & 9.092 & 9.5418 \\ & & & \end{array}$

Fig6BPSTH_monkeyH:

\begin{tabular}{|c|c|c|c|}
\hline & Time_(ms) & Preferred & Anti-preferred \\
\hline 1 & $-29 \overline{5}$ & 11.5663 & 12.3877 \\
\hline 2 & -285 & 11.254 & 12.3001 \\
\hline 3 & -275 & 11.1641 & 12.3482 \\
\hline 4 & -265 & 10.9693 & 12.0029 \\
\hline 5 & -255 & 11.1881 & 12.2428 \\
\hline 6 & -245 & 11.1101 & 11.8269 \\
\hline 7 & -235 & 11.8576 & 12.1127 \\
\hline 8 & -225 & 12.3481 & 12.5379 \\
\hline 9 & -215 & 12.7106 & 13.0319 \\
\hline 10 & -205 & 12.809 & 12.7674 \\
\hline 11 & -195 & 12.9483 & 12.6902 \\
\hline 12 & -185 & 12.3791 & 12.9068 \\
\hline 13 & -175 & 12.3286 & 12.6447 \\
\hline 14 & -165 & 12.2716 & 12.5598 \\
\hline 15 & -155 & 11.6011 & 12.7197 \\
\hline 16 & -145 & 11.5187 & 12.857 \\
\hline 17 & -135 & 11.9878 & 12.2732 \\
\hline 18 & -125 & 11.7352 & 12.8369 \\
\hline 19 & -115 & 11.5802 & 13.0304 \\
\hline 20 & -105 & 11.9894 & 13.3671 \\
\hline 21 & -95 & 12.0602 & 13.1615 \\
\hline 22 & -85 & 11.4849 & 13.8033 \\
\hline 23 & -75 & 11.8249 & 13.4351 \\
\hline 24 & -65 & 11.8066 & 12.8096 \\
\hline 25 & -55 & 11.8017 & 12.8121 \\
\hline 26 & -45 & 12.8926 & 14.3469 \\
\hline 27 & -35 & 15.3671 & 16.0893 \\
\hline 28 & -25 & 16.8595 & 17.4929 \\
\hline 29 & -15 & 18.3013 & 19.1258 \\
\hline
\end{tabular}

\begin{tabular}{|c|c|c|c|}
\hline 30 & -5 & 19.8493 & 20.0658 \\
\hline 31 & 5 & 19.1418 & 18.8956 \\
\hline 32 & 15 & 17.0236 & 17.1792 \\
\hline 33 & 25 & 16.171 & 16.3964 \\
\hline 34 & 35 & 15.8519 & 16.1053 \\
\hline 35 & 45 & 14.7788 & 15.2687 \\
\hline 36 & 55 & 14.688 & 15.1351 \\
\hline 37 & 65 & 14.4671 & 14.8162 \\
\hline 38 & 75 & 13.5413 & 13.7681 \\
\hline 39 & 85 & 12.3828 & 12.2365 \\
\hline 40 & 95 & 12.0204 & 11.3035 \\
\hline 41 & 105 & 11.4334 & 10.6246 \\
\hline 42 & 115 & 11.3928 & 10.1325 \\
\hline 43 & 125 & 11.6468 & 10.3029 \\
\hline 44 & 135 & 11.8158 & 10.5685 \\
\hline 45 & 145 & 11.6137 & 10.4079 \\
\hline 46 & 155 & 11.8852 & 11.033 \\
\hline 47 & 165 & 12.0954 & 11.5655 \\
\hline 48 & 175 & 12.2988 & 11.8583 \\
\hline 49 & 185 & 12.0254 & 12.0402 \\
\hline 50 & 195 & 12.1551 & 12.7955 \\
\hline 51 & 205 & 13.0049 & 13.3282 \\
\hline 52 & 215 & 12.9797 & 13.1936 \\
\hline 53 & 225 & 12.4456 & 13.1169 \\
\hline 54 & 235 & 12.6527 & 12.7632 \\
\hline 55 & 245 & 12.6754 & 12.0553 \\
\hline 56 & 255 & 11.3851 & 11.0027 \\
\hline 57 & 265 & 10.6852 & 10.4788 \\
\hline 58 & 275 & 10.4991 & 9.8746 \\
\hline 59 & 285 & 10.0252 & 9.9294 \\
\hline 60 & 295 & 9.2609 & 9.4357 \\
\hline 61 & 305 & 8.78 & 9.2558 \\
\hline 62 & 315 & 8.6542 & 8.7052 \\
\hline 63 & 325 & 8.5114 & 8.4833 \\
\hline 64 & 335 & 8.2719 & 8.0042 \\
\hline 65 & 345 & 8.2196 & 8.4123 \\
\hline 66 & 355 & 8.769 & 8.1678 \\
\hline 67 & 365 & 9.0587 & 8.7353 \\
\hline 68 & 375 & 8.9361 & 9.3839 \\
\hline 69 & 385 & 9.4108 & 9.8177 \\
\hline 70 & 395 & 9.7387 & 10.0816 \\
\hline 71 & 405 & 9.6731 & 10.8827 \\
\hline 72 & 415 & 9.8723 & 10.8304 \\
\hline 73 & 425 & 9.8973 & 10.475 \\
\hline 74 & 435 & 9.522 & 10.7901 \\
\hline 75 & 445 & 9.5642 & 10.8067 \\
\hline 76 & 455 & 9.806 & 10.9858 \\
\hline 77 & 465 & 9.6302 & 11.0404 \\
\hline 78 & 475 & 9.3996 & 10.9811 \\
\hline 79 & 485 & 8.9485 & 10.2635 \\
\hline 80 & 495 & 8.491 & 9.4706 \\
\hline
\end{tabular}

Fig6CPSTH_monkeyE:

\begin{tabular}{llll} 
& Time_(ms) & \multicolumn{1}{c}{ Preferred } & \multicolumn{1}{c}{ Anti-preferred } \\
1 & -295 & 6.6643 & 6.6146 \\
2 & -285 & 6.8988 & 6.6112 \\
3 & -275 & 6.6082 & 6.3299 \\
4 & -265 & 6.7617 & 6.0975 \\
5 & -255 & 7.0314 & 5.9329 \\
6 & -245 & 7.2826 & 6.0413 \\
7 & -235 & 7.1008 & 5.9828 \\
8 & -225 & 7.5844 & 6.074
\end{tabular}




\begin{tabular}{|c|c|c|c|c|c|c|c|}
\hline 9 & -215 & 7.6387 & 5.8088 & 72 & 415 & 8.7118 & 9.2929 \\
\hline 10 & -205 & 7.4634 & 5.6973 & 73 & 425 & 8.6207 & 9.9291 \\
\hline 11 & -195 & 7.3266 & 5.4195 & 74 & 435 & 8.5544 & 9.7376 \\
\hline 12 & -185 & 7.4496 & 5.3315 & 75 & 445 & 8.5101 & 9.5724 \\
\hline 13 & -175 & 7.2753 & 5.1273 & 76 & 455 & 8.5129 & 9.2552 \\
\hline 14 & -165 & 7.0902 & 5.4637 & 77 & 465 & 7.9738 & 8.6075 \\
\hline 15 & -155 & 7.6209 & 5.5855 & 78 & 475 & 7.8974 & 7.6746 \\
\hline 16 & -145 & 8.06 & 5.657 & 79 & 485 & 7.9896 & 7.1776 \\
\hline 17 & -135 & 7.7107 & 6.1589 & 80 & 495 & 8.0382 & 7.2738 \\
\hline 18 & -125 & 8.0609 & 6.3445 & & & & \\
\hline 19 & -115 & 8.3086 & 6.1937 & \multicolumn{4}{|c|}{ Fig6DPSTH_monkeyE: } \\
\hline 20 & -105 & 8.0064 & 6.3839 & & & & \\
\hline 21 & -95 & 7.5054 & 6.3191 & & Time_(ms) & Preferred & Anti-preferred \\
\hline 22 & -85 & 7.9702 & 6.1537 & 1 & -295 & 7.3263 & 6.0092 \\
\hline 23 & -75 & 7.6618 & 5.944 & 2 & -285 & 7.1993 & 5.8581 \\
\hline 24 & -65 & 7.3229 & 6.1357 & 3 & -275 & 7.3221 & 5.9155 \\
\hline 25 & -55 & 7.5006 & 5.8633 & 4 & -265 & 7.2398 & 6.0509 \\
\hline 26 & -45 & 7.339 & 5.7664 & 5 & -255 & 7.1808 & 6.0675 \\
\hline 27 & -35 & 6.9706 & 5.8804 & 6 & -245 & 6.8598 & 6.5494 \\
\hline 28 & -25 & 7.121 & 6.4077 & 7 & -235 & 7.0029 & 6.6198 \\
\hline 29 & -15 & 7.3895 & 6.453 & 8 & -225 & 7.2188 & 6.7418 \\
\hline 30 & -5 & 6.9433 & 6.5956 & 9 & -215 & 7.4029 & 6.3377 \\
\hline 31 & 5 & 7.2255 & 7.1892 & 10 & -205 & 7.3714 & 6.291 \\
\hline 32 & 15 & 8.0699 & 7.5025 & 11 & -195 & 7.4818 & 5.8766 \\
\hline 33 & 25 & 8.0875 & 7.9244 & 12 & -185 & 7.4089 & 5.9587 \\
\hline 34 & 35 & 8.1852 & 8.6096 & 13 & -175 & 7.328 & 6.0484 \\
\hline 35 & 45 & 8.4253 & 9.3173 & 14 & -165 & 6.989 & 6.3218 \\
\hline 36 & 55 & 8.7357 & 10.0759 & 15 & -155 & 7.0877 & 6.4714 \\
\hline 37 & 65 & 8.8467 & 10.8132 & 16 & -145 & 7.1333 & 6.5399 \\
\hline 38 & 75 & 8.9727 & 11.4437 & 17 & -135 & 6.9511 & 6.1158 \\
\hline 39 & 85 & 9.3911 & 11.5827 & 18 & -125 & 6.7011 & 5.9735 \\
\hline 40 & 95 & 9.5218 & 11.6206 & 19 & -115 & 7.0091 & 5.8028 \\
\hline 41 & 105 & 8.9588 & 10.3391 & 20 & -105 & 6.7226 & 5.8407 \\
\hline 42 & 115 & 8.0015 & 9.2123 & 21 & -95 & 6.5281 & 6.0232 \\
\hline 43 & 125 & 7.4719 & 7.9568 & 22 & -85 & 6.6912 & 6.3836 \\
\hline 44 & 135 & 6.5863 & 7.216 & 23 & -75 & 6.8024 & 6.4182 \\
\hline 45 & 145 & 5.9605 & 6.6486 & 24 & -65 & 7.1838 & 6.4205 \\
\hline 46 & 155 & 6.0772 & 6.9159 & 25 & -55 & 7.5742 & 6.3737 \\
\hline 47 & 165 & 6.4683 & 6.5154 & 26 & -45 & 7.3254 & 5.9667 \\
\hline 48 & 175 & 6.4319 & 6.6233 & 27 & -35 & 7.2862 & 5.7165 \\
\hline 49 & 185 & 6.9702 & 6.6884 & 28 & -25 & 7.5089 & 6.0069 \\
\hline 50 & 195 & 7.8888 & 7.0692 & 29 & -15 & 7.0667 & 6.3652 \\
\hline 51 & 205 & 8.2041 & 7.3927 & 30 & -5 & 6.9439 & 6.3091 \\
\hline 52 & 215 & 8.5193 & 8.6205 & 31 & 5 & 7.1251 & 7.0287 \\
\hline 53 & 225 & 9.2135 & 8.9033 & 32 & 15 & 7.3156 & 8.2333 \\
\hline 54 & 235 & 9.2101 & 9.1364 & 33 & 25 & 7.3356 & 8.5191 \\
\hline 55 & 245 & 9.3411 & 8.8504 & 34 & 35 & 7.9797 & 8.6236 \\
\hline 56 & 255 & 9.6215 & 8.7682 & 35 & 45 & 8.6282 & 9.1341 \\
\hline 57 & 265 & 9.6523 & 8.0169 & 36 & 55 & 8.989 & 9.2094 \\
\hline 58 & 275 & 9.3308 & 7.6784 & 37 & 65 & 9.257 & 9.3028 \\
\hline 59 & 285 & 9.0416 & 7.322 & 38 & 75 & 9.858 & 9.4337 \\
\hline 60 & 295 & 8.6492 & 7.4794 & 39 & 85 & 10.0311 & 9.8038 \\
\hline 61 & 305 & 8.2751 & 7.5116 & 40 & 95 & 9.5512 & 9.7956 \\
\hline 62 & 315 & 8.1024 & 8.0346 & 41 & 105 & 9.1392 & 9.2534 \\
\hline 63 & 325 & 7.9146 & 7.8261 & 42 & 115 & 8.3007 & 8.2236 \\
\hline 64 & 335 & 7.9377 & 8.0957 & 43 & 125 & 7.5406 & 7.6825 \\
\hline 65 & 345 & 8.1548 & 7.9721 & 44 & 135 & 6.3726 & 7.0336 \\
\hline 66 & 355 & 8.123 & 7.8011 & 45 & 145 & 6.0866 & 6.3635 \\
\hline 67 & 365 & 7.6661 & 7.3671 & 46 & 155 & 5.8521 & 6.2003 \\
\hline 68 & 375 & 7.8214 & 7.6644 & 47 & 165 & 6.0582 & 5.9775 \\
\hline 69 & 385 & 8.1432 & 7.9759 & 48 & 175 & 5.7644 & 6.0738 \\
\hline 70 & 395 & 7.958 & 8.2627 & 49 & 185 & 6.1287 & 6.3201 \\
\hline 71 & 405 & 8.3248 & 8.6735 & 50 & 195 & 6.3438 & 6.6735 \\
\hline
\end{tabular}




\begin{tabular}{|c|c|c|c|}
\hline 51 & 205 & 6.8641 & 6.99 \\
\hline 52 & 215 & 7.4355 & 7.7104 \\
\hline 53 & 225 & 8.0424 & 7.7678 \\
\hline 54 & 235 & 8.2941 & 7.9602 \\
\hline 55 & 245 & 8.6153 & 8.3309 \\
\hline 56 & 255 & 8.8686 & 8.5336 \\
\hline 57 & 265 & 8.9405 & 8.5434 \\
\hline 58 & 275 & 8.9151 & 8.4044 \\
\hline 59 & 285 & 8.7116 & 8.0481 \\
\hline 60 & 295 & 8.3872 & 7.9507 \\
\hline 61 & 305 & 8.2758 & 7.4878 \\
\hline 62 & 315 & 7.9481 & 7.2058 \\
\hline 63 & 325 & 7.7684 & 7.6841 \\
\hline 64 & 335 & 7.8779 & 8.1843 \\
\hline 65 & 345 & 8.1954 & 8.0559 \\
\hline 66 & 355 & 8.1697 & 8.3099 \\
\hline 67 & 365 & 8.3353 & 8.3384 \\
\hline 68 & 375 & 8.4553 & 7.5733 \\
\hline 69 & 385 & 8.3713 & 7.6411 \\
\hline 70 & 395 & 8.0566 & 8.0946 \\
\hline 71 & 405 & 8.2075 & 8.5513 \\
\hline 72 & 415 & 8.1664 & 9.2172 \\
\hline 73 & 425 & 8.0728 & 9.9939 \\
\hline 74 & 435 & 8.1691 & 10.0004 \\
\hline 75 & 445 & 8.1906 & 9.6854 \\
\hline 76 & 455 & 8.0378 & 9.4982 \\
\hline 77 & 465 & 7.8285 & 8.6919 \\
\hline 78 & 475 & 8.1996 & 8.6588 \\
\hline 79 & 485 & 8.253 & 8.4706 \\
\hline 80 & 495 & 8.3933 & 8.3221 \\
\hline
\end{tabular}

Fig6EPSTH_monkeyH:

Time_(ms) Mean_difference Cl_of_difference

$\begin{array}{llll}1 & -295 & & \\ 2 & -285 & -1.1913 & 2.0819 \\ 3 & -275 & -0.154861 & 1.6891 \\ 4 & -265 & -0.047027 & 1.5015 \\ 5 & -255 & 0.71556 & 1.4058 \\ 6 & -245 & 1.1622 & 1.5964 \\ 7 & -235 & 0.88356 & 1.6027 \\ 8 & -225 & 0.92277 & 1.5517 \\ 9 & -215 & 0.31532 & 1.5492 \\ 10 & -205 & -0.47627 & 1.31 \\ 11 & -195 & -0.83398 & 1.3591 \\ 12 & -185 & -0.94246 & 1.5273 \\ 13 & -175 & -1.9597 & 1.6314 \\ 14 & -165 & -0.96963 & 1.5871 \\ 15 & -155 & -0.70902 & 1.5915 \\ 16 & -145 & -0.80982 & 1.4152 \\ 17 & -135 & -0.18382 & 1.3235 \\ 18 & -125 & 0.79886 & 1.4354 \\ 19 & -115 & 0.82917 & 1.6509 \\ 20 & -105 & 0.74753 & 1.6812 \\ 21 & -95 & 0.57221 & 1.736 \\ 22 & -85 & -0.36412 & 2.0907 \\ 23 & -75 & -0.91036 & 2.355 \\ 24 & -65 & -1.6249 & 2.5437 \\ 25 & -55 & -1.5757 & 2.6306 \\ 26 & -45 & -1.8418 & 2.4895 \\ 27 & -35 & -1.5295 & 1.8721 \\ 28 & -25 & -1.4474 & 1.7151\end{array}$

$29-1$

$30-5$

315

3215

3325

3435

$35 \quad 45$

3655

$37 \quad 65$

3875

3985

$40 \quad 95$

41105

$42 \quad 115$

$43 \quad 125$

$44 \quad 135$

$45 \quad 145$

$46 \quad 155$

$47 \quad 165$

$48 \quad 175$

$49 \quad 185$

$50 \quad 195$

51205

$52 \quad 215$

$53 \quad 225$

$54 \quad 235$

$55 \quad 245$

$56 \quad 255$

$\begin{array}{ll}57 & 265\end{array}$

$58 \quad 275$

59285

60295

61305

$62 \quad 315$

$63 \quad 325$

$64 \quad 335$

$65 \quad 345$

$66 \quad 355$

$67 \quad 365$

$68 \quad 375$

69385

$70 \quad 395$

71405

$72 \quad 415$

$\begin{array}{ll}73 & 425\end{array}$

$74 \quad 435$

$75 \quad 445$

$\begin{array}{ll}76 & 455\end{array}$

$77 \quad 465$
78

\begin{tabular}{ll}
$78 \quad 475$ \\
\hline
\end{tabular}

$79 \quad 485$

$80 \quad 495$

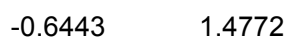

$-0.03844 \quad 1.3392$

$0.79246 \quad 1.0842$

$1.0071 \quad 1.3926$

$1.6275 \quad 1.3723$

$1.3393 \quad 1.3352$

$0.29402 \quad 1.5363$

$0.43194 \quad 1.6353$

$0.46376 \quad 1.4742$

$0.22224 \quad 1.3401$

$0.0064073 \quad 1.439$

$0.58193 \quad 1.2885$

$0.019306 \quad 1.4807$

$0.15331 \quad 1.6179$

$-0.17867 \quad 1.7002$

$0.49678 \quad 1.7945$

$-0.22865 \quad 1.6496$

$-0.39767 \quad 1.7536$

$0.016985 \quad 1.6604$

$0.33457 \quad 1.4282$

$-0.26144 \quad 1.5741$

$0.041268 \quad 1.6871$

$0.93068 \quad 1.7831$

$0.92041 \quad 1.8337$

$1.5584 \quad 1.5929$

$1.8075 \quad 1.5052$

$2.3307 \quad 1.5517$

$1.7555 \quad 1.7504$

$0.66566 \quad 1.8751$

$-0.079706 \quad 1.7855$

$-0.05392 \quad 1.4321$

$0.040596 \quad 1.2919$

$0.57357 \quad 1.2703$

$1.3177 \quad 1.4252$

$1.5793 \quad 1.4723$

$1.7309 \quad 1.6964$

$1.3155 \quad 1.6601$

$0.88105 \quad 1.8885$

$1.2563 \quad 1.728$

$1.1346 \quad 1.533$

$0.45918 \quad 1.5011$

$0.39869 \quad 1.4975$

$0.029005 \quad 1.3402$

$-0.081382 \quad 1.6057$

$-0.34119 \quad 1.3402$

$0.042809 \quad 1.2172$

$0.1935 \quad 1.2728$

$0.44786 \quad 1.2826$

$-0.25635 \quad 1.4849$

$-0.19581 \quad 1.4618$

$-0.20789 \quad 1.4992$

$\begin{array}{ll}-0.44982 & 1.3567\end{array}$

\section{Fig6FPSTH_monkeyH:}

Time_(ms) $\mathrm{Cl}$ of_difference

$\begin{array}{llll}1 & -295 & -0.82143 & 1.5739 \\ 2 & -285 & -1.0461 & 1.626 \\ 3 & -275 & -1.1841 & 1.8388 \\ 4 & -265 & -1.0336 & 1.7483 \\ 5 & -255 & -1.0547 & 1.4687\end{array}$




\begin{tabular}{|c|c|c|c|c|c|c|c|}
\hline 6 & -245 & -0.71677 & 1.6205 & 69 & 385 & -0.40691 & 1.692 \\
\hline 7 & -235 & -0.25511 & 1.719 & 70 & 395 & -0.34288 & 1.1756 \\
\hline 8 & -225 & -0.18973 & 1.7852 & 71 & 405 & -1.2097 & 1.3038 \\
\hline 9 & -215 & -0.32125 & 1.7812 & 72 & 415 & -0.95809 & 1.306 \\
\hline 10 & -205 & 0.041609 & 1.92 & 73 & 425 & -0.57768 & 1.7342 \\
\hline 11 & -195 & 0.25805 & 1.9573 & 74 & 435 & -1.2682 & 1.8474 \\
\hline 12 & -185 & -0.52772 & 1.8235 & 75 & 445 & -1.2425 & 1.6815 \\
\hline 13 & -175 & -0.3161 & 2.1455 & 76 & 455 & -1.1797 & 1.5221 \\
\hline 14 & -165 & -0.28816 & 1.999 & 77 & 465 & -1.4102 & 1.4435 \\
\hline 15 & -155 & -1.1186 & 2.092 & 78 & 475 & -1.5815 & 1.6584 \\
\hline 16 & -145 & -1.3383 & 2.3765 & 79 & 485 & -1.315 & 1.4764 \\
\hline 17 & -135 & -0.28538 & 2.2644 & 80 & 495 & -0.97963 & 1.3297 \\
\hline 18 & -125 & -1.1016 & 2.7065 & & & & \\
\hline 19 & -115 & -1.4503 & 2.7586 & \multicolumn{4}{|c|}{ Fig6GPSTH_monkeyE: } \\
\hline 20 & -105 & -1.3777 & 2.7412 & \multirow{2}{*}{\multicolumn{4}{|c|}{ Mean_difference }} \\
\hline 21 & -95 & -1.1013 & 1.9694 & & & & \\
\hline 22 & -85 & -2.3183 & 1.8975 & \multicolumn{4}{|c|}{ Cl_of_difference } \\
\hline 23 & -75 & -1.6102 & 1.7368 & 1 & -295 & 0.049706 & 1.2363 \\
\hline 24 & -65 & -1.003 & 1.4691 & 2 & -285 & 0.28762 & 1.2486 \\
\hline 25 & -55 & -1.0104 & 1.545 & 3 & -275 & 0.27833 & 1.1219 \\
\hline 26 & -45 & -1.4543 & 1.7344 & 4 & -265 & 0.66422 & 1.1084 \\
\hline 27 & -35 & -0.72228 & 1.6397 & 5 & -255 & 1.0984 & 1.1166 \\
\hline 28 & -25 & -0.63339 & 1.3791 & 6 & -245 & 1.2413 & 1.2024 \\
\hline 29 & -15 & -0.82448 & 1.0342 & 7 & -235 & 1.118 & 1.2154 \\
\hline 30 & -5 & -0.21653 & 1.0012 & 8 & -225 & 1.5104 & 1.1523 \\
\hline 31 & 5 & 0.24621 & 1.7168 & 9 & -215 & 1.8298 & 1.3464 \\
\hline 32 & 15 & -0.15563 & 1.8732 & 10 & -205 & 1.7661 & 1.2508 \\
\hline 33 & 25 & -0.22544 & 1.5742 & 11 & -195 & 1.9071 & 1.3287 \\
\hline 34 & 35 & -0.25336 & 1.608 & 12 & -185 & 2.1181 & 1.3613 \\
\hline 35 & 45 & -0.4899 & 1.2068 & 13 & -175 & 2.148 & 1.2706 \\
\hline 36 & 55 & -0.44703 & 0.94826 & 14 & -165 & 1.6264 & 1.2995 \\
\hline 37 & 65 & -0.34906 & 0.84669 & 15 & -155 & 2.0354 & 1.5476 \\
\hline 38 & 75 & -0.22685 & 0.85431 & 16 & -145 & 2.403 & 1.6117 \\
\hline 39 & 85 & 0.1463 & 1.0161 & 17 & -135 & 1.5517 & 1.1675 \\
\hline 40 & 95 & 0.71689 & 1.2357 & 18 & -125 & 1.7164 & 1.1355 \\
\hline 41 & 105 & 0.8088 & 1.3559 & 19 & -115 & 2.1149 & 1.3407 \\
\hline 42 & 115 & 1.2603 & 1.4736 & 20 & -105 & 1.6225 & 1.1891 \\
\hline 43 & 125 & 1.3439 & 1.7318 & 21 & -95 & 1.1863 & 1.3267 \\
\hline 44 & 135 & 1.2473 & 1.7642 & 22 & -85 & 1.8164 & 1.2614 \\
\hline 45 & 145 & 1.2058 & 1.69 & 23 & -75 & 1.7178 & 1.4113 \\
\hline 46 & 155 & 0.85215 & 1.448 & 24 & -65 & 1.1872 & 1.2645 \\
\hline 47 & 165 & 0.52995 & 1.3687 & 25 & -55 & 1.6373 & 1.5288 \\
\hline 48 & 175 & 0.44047 & 1.5033 & 26 & -45 & 1.5727 & 1.4669 \\
\hline 49 & 185 & -0.014807 & 1.3875 & 27 & -35 & 1.0902 & 1.4668 \\
\hline 50 & 195 & -0.64036 & 1.4956 & 28 & -25 & 0.71336 & 1.3195 \\
\hline 51 & 205 & -0.32336 & 1.3009 & 29 & -15 & 0.93648 & 1.4615 \\
\hline 52 & 215 & -0.21384 & 1.3636 & 30 & -5 & 0.34763 & 1.2495 \\
\hline 53 & 225 & -0.67128 & 1.3232 & 31 & 5 & 0.036328 & 1.1483 \\
\hline 54 & 235 & -0.11054 & 1.0772 & 32 & 15 & 0.56736 & 0.9814 \\
\hline 55 & 245 & 0.6201 & 1.4822 & 33 & 25 & 0.16306 & 0.9913 \\
\hline 56 & 255 & 0.38245 & 1.8129 & 34 & 35 & -0.42441 & 1.0802 \\
\hline 57 & 265 & 0.20632 & 1.7835 & 35 & 45 & -0.89197 & 1.052 \\
\hline 58 & 275 & 0.62444 & 1.726 & 36 & 55 & -1.3402 & 1.4245 \\
\hline 59 & 285 & 0.095831 & 1.851 & 37 & 65 & -1.9665 & 1.4392 \\
\hline 60 & 295 & -0.17478 & 1.4951 & 38 & 75 & -2.471 & 1.538 \\
\hline 61 & 305 & -0.47578 & 1.3597 & 39 & 85 & -2.1917 & 1.5244 \\
\hline 62 & 315 & -0.051024 & 1.5439 & 40 & 95 & -2.0988 & 1.372 \\
\hline 63 & 325 & 0.028066 & 1.5328 & 41 & 105 & -1.3803 & 1.2341 \\
\hline 64 & 335 & 0.26775 & 1.4487 & 42 & 115 & -1.2108 & 1.1972 \\
\hline 65 & 345 & -0.19264 & 1.6687 & 43 & 125 & -0.4849 & 1.1398 \\
\hline 66 & 355 & 0.60118 & 1.9656 & 44 & 135 & -0.62971 & 1.5476 \\
\hline 67 & 365 & 0.32341 & 1.9011 & 45 & 145 & -0.6881 & 1.5188 \\
\hline 68 & 375 & -0.44776 & 1.7966 & 46 & 155 & -0.83873 & 1.5019 \\
\hline
\end{tabular}




$\begin{array}{llll}47 & 165 & -0.04713 & 1.4841 \\ 48 & 175 & -0.19137 & 1.2127 \\ 49 & 185 & 0.28178 & 1.2928 \\ 50 & 195 & 0.81952 & 1.1798 \\ 51 & 205 & 0.81139 & 1.2985 \\ 52 & 215 & -0.1012 & 1.467 \\ 53 & 225 & 0.31023 & 1.5437 \\ 54 & 235 & 0.073632 & 1.6404 \\ 55 & 245 & 0.49063 & 1.5989 \\ 56 & 255 & 0.85332 & 1.4903 \\ 57 & 265 & 1.6354 & 1.4404 \\ 58 & 275 & 1.6524 & 1.3701 \\ 59 & 285 & 1.7196 & 1.2229 \\ 60 & 295 & 1.1697 & 1.4193 \\ 61 & 305 & 0.76354 & 1.4041 \\ 62 & 315 & 0.06782 & 1.5203 \\ 63 & 325 & 0.088547 & 1.3976 \\ 64 & 335 & -0.15798 & 1.5121 \\ 65 & 345 & 0.18271 & 1.4071 \\ 66 & 355 & 0.32186 & 1.4976 \\ 67 & 365 & 0.29896 & 1.4213 \\ 68 & 375 & 0.15699 & 1.4675 \\ 69 & 385 & 0.16733 & 1.3509 \\ 70 & 395 & -0.30471 & 1.3449 \\ 71 & 405 & -0.34866 & 1.6107 \\ 72 & 415 & -0.58114 & 1.5905 \\ 73 & 425 & -1.3084 & 1.6999 \\ 74 & 435 & -1.1832 & 1.5237 \\ 75 & 445 & -1.0623 & 1.2557 \\ 76 & 455 & -0.74233 & 1.0024 \\ 77 & 465 & -0.63371 & 1.0958 \\ 78 & 475 & 0.22273 & 1.2199 \\ 79 & 485 & 0.81206 & 1.4014 \\ 80 & 495 & 0.76435 & 1.3316\end{array}$

Fig6HPSTH_monkeyE:

Time_(ms) Mean_difference Cl_of_difference

$\begin{array}{clll}1 & -295 & 1.3171 & 1.1571 \\ 2 & -285 & 1.3411 & 1.1175 \\ 3 & -275 & 1.4066 & 1.0946 \\ 4 & -265 & 1.1889 & 1.0029 \\ 5 & -255 & 1.1133 & 1.2042 \\ 6 & -245 & 0.31041 & 1.1511 \\ 7 & -235 & 0.38315 & 1.2283 \\ 8 & -225 & 0.47697 & 0.98795 \\ 9 & -215 & 1.0652 & 1.0052 \\ 10 & -205 & 1.0805 & 0.9794 \\ 11 & -195 & 1.6052 & 1.0829 \\ 12 & -185 & 1.4502 & 1.1536 \\ 13 & -175 & 1.2796 & 1.1972 \\ 14 & -165 & 0.6672 & 1.1713 \\ 15 & -155 & 0.61634 & 1.155 \\ 16 & -145 & 0.59344 & 1.1493 \\ 17 & -135 & 0.83523 & 0.96163 \\ 18 & -125 & 0.72759 & 1.1239 \\ 19 & -115 & 1.2063 & 1.4272 \\ 20 & -105 & 0.88187 & 1.3082 \\ 21 & -95 & 0.50486 & 1.5757 \\ 22 & -85 & 0.30762 & 1.5076 \\ 23 & -75 & 0.38412 & 1.4283 \\ 24 & -65 & 0.76326 & 1.3824\end{array}$

\begin{tabular}{|c|c|c|c|}
\hline 25 & -55 & 1.2005 & 1.4944 \\
\hline 26 & -45 & 1.3587 & 1.6424 \\
\hline 27 & -35 & 1.5697 & 1.7707 \\
\hline 28 & -25 & 1.5021 & 1.5458 \\
\hline 29 & -15 & 0.70152 & 1.5315 \\
\hline 30 & -5 & 0.6348 & 1.4645 \\
\hline 31 & 5 & 0.096418 & 1.3479 \\
\hline 32 & 15 & -0.91771 & 1.5312 \\
\hline 33 & 25 & -1.1835 & 1.638 \\
\hline 34 & 35 & -0.64397 & 1.4427 \\
\hline 35 & 45 & -0.50592 & 1.28 \\
\hline 36 & 55 & -0.2204 & 1.4263 \\
\hline 37 & 65 & -0.045706 & 1.5443 \\
\hline 38 & 75 & 0.42431 & 1.6978 \\
\hline 39 & 85 & 0.22737 & 1.6632 \\
\hline 40 & 95 & -0.24435 & 1.4911 \\
\hline 41 & 105 & -0.11421 & 1.5859 \\
\hline 42 & 115 & 0.077109 & 1.6084 \\
\hline 43 & 125 & -0.14188 & 1.552 \\
\hline 44 & 135 & -0.66104 & 1.1139 \\
\hline 45 & 145 & -0.27699 & 1.0982 \\
\hline 46 & 155 & -0.34819 & 1.0996 \\
\hline 47 & 165 & 0.080735 & 1.2047 \\
\hline 48 & 175 & -0.30945 & 1.2427 \\
\hline 49 & 185 & -0.19134 & 1.0993 \\
\hline 50 & 195 & -0.32975 & 1.076 \\
\hline 51 & 205 & -0.12589 & 1.1627 \\
\hline 52 & 215 & -0.27486 & 1.2006 \\
\hline 53 & 225 & 0.27464 & 1.2421 \\
\hline 54 & 235 & 0.33393 & 1.8051 \\
\hline 55 & 245 & 0.28446 & 1.7929 \\
\hline 56 & 255 & 0.33501 & 1.6519 \\
\hline 57 & 265 & 0.39703 & 1.5994 \\
\hline 58 & 275 & 0.51068 & 1.476 \\
\hline 59 & 285 & 0.66342 & 1.2678 \\
\hline 60 & 295 & 0.43651 & 1.1688 \\
\hline 61 & 305 & 0.78801 & 1.485 \\
\hline 62 & 315 & 0.74225 & 1.4469 \\
\hline 63 & 325 & 0.084311 & 1.2626 \\
\hline 64 & 335 & -0.30636 & 1.3852 \\
\hline 65 & 345 & 0.13952 & 1.8182 \\
\hline 66 & 355 & -0.14019 & 1.5876 \\
\hline 67 & 365 & -0.0031619 & 1.3511 \\
\hline 68 & 375 & 0.88206 & 1.1899 \\
\hline 69 & 385 & 0.73018 & 1.1517 \\
\hline 70 & 395 & -0.037977 & 1.1008 \\
\hline 71 & 405 & -0.34383 & 1.3039 \\
\hline 72 & 415 & -1.0508 & 1.5609 \\
\hline 73 & 425 & -1.9211 & 1.6116 \\
\hline 74 & 435 & -1.8313 & 1.7256 \\
\hline 75 & 445 & -1.4947 & 1.6818 \\
\hline 76 & 455 & -1.4604 & 1.8487 \\
\hline 77 & 465 & -0.86338 & 1.6711 \\
\hline 78 & 475 & -0.45918 & 1.4149 \\
\hline 79 & 485 & -0.21754 & 1.271 \\
\hline 80 & 495 & 0.071217 & 1.3048 \\
\hline
\end{tabular}


S6 Data. The data presented in S1 Fig.

Note:

FigS1PSTH_monkeyH.txt contains the values for the population average PSTHs for monkey $\mathrm{H}$ in Figure S1 FigS1PSTH_monkeyE.txt contains the values for the population average PSTHs for monkey E in Figure S1

FigS1PSTH_monkeyH:

Time (ms) Mean sensory response SEM remapped response

0.01848

$2-285$

0.0075147

0.0050541

$-0.0028804$

$4-265$

0.0060576

$\begin{array}{ll}5 & -255 \\ 6 & -245\end{array}$

$6-245$

$7-235$

$8-225$

$9-215$

$10-205$

$11-195$

$12-185$

$13-175$

$14-165$

$15-155$

$16-145$

$\begin{array}{ll}17 & -135\end{array}$

$18-125$

$\begin{array}{ll}19 & -115\end{array}$

$20-105$

$21-95$

$\begin{array}{lll}22 & -85\end{array}$

$23-75$

$24-65$

$25-55$

$26-45$

$\begin{array}{ll}27 & -35\end{array}$

$28-25$

$\begin{array}{ll}29 & -15\end{array}$

$30-5$

315

$32 \quad 15$

$33 \quad 25$

$34 \quad 35$

$35 \quad 45$

$36 \quad 55$

$37 \quad 65$

3875

$39 \quad 85$

4095

$41 \quad 105$

$42 \quad 115$

$43 \quad 125$

$44 \quad 135$

$45 \quad 145$

$46 \quad 155$

$47 \quad 165$

$48 \quad 175$

$49 \quad 185$

$50 \quad 195$

51205

$-0.012807$

$-0.0083755$

$-0.011824$

$-0.0087666$

$-0.0095901$

$-0.013404$

$-0.0051188$

$-0.00051309$

$-0.0050449$

$-0.0097405$

$-0.00041036$

$-0.0081347$

$-0.0050513$

$-0.0031991$

$-0.0085734$

$-0.0012513$

0.0058914

0.0018651

$-0.00024995$

0.014806

0.014026

0.0083577

0.013476

0.021143

0.010375

0.052522

0.18534

0.35889

0.5141

0.69684

0.83927

0.90235

0.94303

0.99791

1.0031

0.99645

0.97456

0.92663

0.86893

0.83548

0.80159

0.77343

0.74514

0.71274

0.68394

0.66135

SEM_sensory_response Mean_remapped_response

0.0087484

0.0090643

0.0085317

0.0090926

0.010667

0.010842

0.0091578

0.0099829

0.008309

0.0090808

0.010736

0.011764

0.012575

0.011759

0.010591

0.010465

0.0098606

0.013589

0.012108

0.012993

0.013528

0.016683

0.016441

0.01691

0.017032

0.01521

0.012537

0.0096147

0.0134

0.011478

0.018569

0.041391

0.059491

0.070269

0.078485

0.083345

0.083165

0.085768

0.093135

0.096868

0.096199

0.094252

0.090222

0.085549

0.083552

0.080654

0.077761

0.074534

0.071131

0.068797

0.069996
0.057926

0.062388

$-0.026778 \quad 0.053476$

$0.015623 \quad 0.066477$

$0.04118 \quad 0.076049$

$0.0047123 \quad 0.069882$

$0.023912 \quad 0.061616$

$0.048778 \quad 0.063378$

$0.0012119 \quad 0.066929$

$-0.051512 \quad 0.077712$

$-0.030814 \quad 0.091626$

$-0.11122 \quad 0.083358$

$-0.16951 \quad 0.085243$

$-0.12079 \quad 0.080888$

$-0.076397 \quad 0.083383$

$-0.094657 \quad 0.10419$

$-0.058781 \quad 0.10079$

$-0.1115$

$-0.043681$

0.10384

0.11291

$0.0035871 \quad 0.10499$

$0.020861 \quad 0.10184$

$0.048439 \quad 0.086472$

0.2013

0.16849

0.085137

0.044095

0.046255

$-0.092232$

$-0.10793$

0.032845

0.15078

0.19397

0.33438

0.49333

0.5135

0.6232

0.80001

0.89191

0.9633

1.0265

1.0124

0.91089

0.8947

0.71295

0.55538

0.45657

0.36548

0.27501

0.25509
0.094872

0.10024

0.088044

0.089774

0.098309

0.09289

0.10285

0.099684

0.11409

0.12614

0.14372

0.19983

0.20227

0.22274

0.24323

0.25078

0.25025

0.24553

0.23023

0.20635

0.20642

0.19675

0.17328

0.16867

0.14387

0.12492

0.12674 


$\begin{array}{llllll}52 & 215 & 0.65593 & 0.069695 & 0.32982 & 0.1115 \\ 53 & 225 & 0.64393 & 0.068822 & 0.30676 & 0.12063 \\ 54 & 235 & 0.64353 & 0.067952 & 0.36831 & 0.10197 \\ 55 & 245 & 0.66145 & 0.068281 & 0.38609 & 0.10029 \\ 56 & 255 & 0.67317 & 0.066136 & 0.48482 & 0.11705 \\ 57 & 265 & 0.66316 & 0.064342 & 0.44795 & 0.12302 \\ 58 & 275 & 0.68471 & 0.066538 & 0.50496 & 0.1563 \\ 59 & 285 & 0.69092 & 0.068363 & 0.52067 & 0.17338 \\ 60 & 295 & 0.6916 & 0.06791 & 0.47896 & 0.18086 \\ 61 & 305 & 0.68185 & 0.067732 & 0.37493 & 0.15343 \\ 62 & 315 & 0.67624 & 0.066812 & 0.3238 & 0.12536 \\ 63 & 325 & 0.65989 & 0.065315 & 0.24765 & 0.11748 \\ 64 & 335 & 0.65519 & 0.064465 & 0.15065 & 0.10423 \\ 65 & 345 & 0.65317 & 0.065278 & 0.098227 & 0.08998 \\ 66 & 355 & 0.64843 & 0.063348 & 0.041072 & 0.096331 \\ 67 & 365 & 0.64817 & 0.064423 & 0.0059498 & 0.10999 \\ 68 & 375 & 0.64955 & 0.063965 & 0.033064 & 0.099511 \\ 69 & 385 & 0.6509 & 0.065023 & 0.050893 & 0.10366 \\ 70 & 395 & 0.6353 & 0.062316 & 0.070514 & 0.10261 \\ 71 & 405 & 0.61958 & 0.060366 & 0.039878 & 0.092901 \\ 72 & 415 & 0.61185 & 0.058786 & 0.074315 & 0.082662 \\ 73 & 425 & 0.59564 & 0.056829 & 0.044805 & 0.091804 \\ 74 & 435 & 0.59533 & 0.05575 & 0.064729 & 0.081799 \\ 75 & 445 & 0.60091 & 0.056319 & 0.11625 & 0.093831 \\ 76 & 455 & 0.61977 & 0.056768 & 0.23996 & 0.082316 \\ 77 & 465 & 0.62337 & 0.056353 & 0.23737 & 0.079022 \\ 78 & 475 & 0.62812 & 0.057333 & 0.23624 & 0.070102 \\ 79 & 485 & 0.62523 & 0.057231 & 0.16445 & 0.043497 \\ 80 & 495 & 0.61608 & 0.057994 & 0.11116 & 0.053274\end{array}$

FigS1PSTH_monkeyE:

Time_(ms) Mean_sensory_response SEM_remapped_response

$\begin{array}{cllccc}1 & -295 & -0.0062942 & 0.012258 & 0.19539 & 0.23923 \\ 2 & -285 & -0.0063491 & 0.011375 & 0.20837 & 0.22901 \\ 3 & -275 & -0.0027549 & 0.011101 & 0.0913 & 0.23306 \\ 4 & -265 & -0.00022307 & 0.010557 & 0.089795 & 0.22772 \\ 5 & -255 & -0.0041986 & 0.010758 & -0.10005 & 0.21688 \\ 6 & -245 & -0.0046777 & 0.0094813 & -0.022359 & 0.22324 \\ 7 & -235 & -0.0046842 & 0.0098708 & -0.13587 & 0.22414 \\ 8 & -225 & -0.010737 & 0.01222 & -0.30034 & 0.30493 \\ 9 & -215 & -0.0072903 & 0.010751 & -0.3657 & 0.2815 \\ 10 & -205 & 0.0029717 & 0.011351 & -0.21887 & 0.26833 \\ 11 & -195 & 0.0067067 & 0.0096024 & -0.33954 & 0.28521 \\ 12 & -185 & 0.0088678 & 0.010257 & -0.23041 & 0.28349 \\ 13 & -175 & 0.015081 & 0.0086137 & -0.022313 & 0.21181 \\ 14 & -165 & 0.01836 & 0.009293 & 0.066552 & 0.21507 \\ 15 & -155 & 0.014006 & 0.0081962 & 0.1924 & 0.2634 \\ 16 & -145 & 0.0076278 & 0.0099851 & 0.3 & 0.30503 \\ 17 & -135 & 0.0047657 & 0.0094554 & 0.26634 & 0.24683 \\ 18 & -125 & -0.0011722 & 0.0092663 & 0.24408 & 0.24212 \\ 19 & -115 & -0.010218 & 0.0088895 & 0.17961 & 0.21416 \\ 20 & -105 & -0.0071747 & 0.0094816 & 0.15861 & 0.2138 \\ 21 & -95 & -0.0026789 & 0.0096664 & 0.072437 & 0.22523 \\ 22 & -85 & -0.0016281 & 0.011736 & 0.13953 & 0.21879 \\ 23 & -75 & 5.1622 e-05 & 0.01483 & 0.00095034 & 0.25546 \\ 24 & -65 & -0.0038675 & 0.015453 & -0.086454 & 0.24237 \\ 25 & -55 & -0.0062266 & 0.012916 & -0.22783 & 0.19438 \\ 26 & -45 & 0.00032782 & 0.012479 & -0.13174 & 0.19402 \\ 27 & -35 & -0.0080286 & 0.0098145 & -0.15397 & 0.22652 \\ 28 & -25 & -0.0047772 & 0.0096986 & -0.014821 & 0.22211\end{array}$




\begin{tabular}{|c|c|c|c|c|c|}
\hline 29 & -15 & 0.0028886 & 0.012432 & 0.085615 & 0.27287 \\
\hline 30 & -5 & 0.011325 & 0.0148 & 0.059305 & 0.24531 \\
\hline 31 & 5 & 0.073296 & 0.024313 & 0.21045 & 0.25776 \\
\hline 32 & 15 & 0.22874 & 0.035417 & 0.1996 & 0.21862 \\
\hline 33 & 25 & 0.4259 & 0.051491 & 0.12434 & 0.21302 \\
\hline 34 & 35 & 0.65032 & 0.068949 & 0.12816 & 0.21694 \\
\hline 35 & 45 & 0.84363 & 0.085614 & 0.17563 & 0.24202 \\
\hline 36 & 55 & 0.97251 & 0.094725 & 0.27191 & 0.25214 \\
\hline 37 & 65 & 0.98825 & 0.096767 & 0.55958 & 0.29421 \\
\hline 38 & 75 & 0.92801 & 0.090911 & 0.69781 & 0.30155 \\
\hline 39 & 85 & 0.83392 & 0.091083 & 0.75678 & 0.30194 \\
\hline 40 & 95 & 0.75599 & 0.088526 & 0.93394 & 0.34351 \\
\hline 41 & 105 & 0.68859 & 0.08321 & 0.60722 & 0.32607 \\
\hline 42 & 115 & 0.64771 & 0.079383 & 0.36991 & 0.31986 \\
\hline 43 & 125 & 0.63243 & 0.074325 & 0.22961 & 0.26404 \\
\hline 44 & 135 & 0.62917 & 0.070373 & 0.17081 & 0.20612 \\
\hline 45 & 145 & 0.62349 & 0.066234 & 0.14461 & 0.20871 \\
\hline 46 & 155 & 0.62238 & 0.065265 & 0.23985 & 0.25471 \\
\hline 47 & 165 & 0.62862 & 0.067664 & 0.21906 & 0.2041 \\
\hline 48 & 175 & 0.62934 & 0.068192 & 0.35227 & 0.21037 \\
\hline 49 & 185 & 0.62433 & 0.067762 & 0.5158 & 0.2373 \\
\hline 50 & 195 & 0.62239 & 0.068172 & 0.56405 & 0.25231 \\
\hline 51 & 205 & 0.60109 & 0.069212 & 0.58294 & 0.28753 \\
\hline 52 & 215 & 0.57602 & 0.064043 & 0.87504 & 0.30961 \\
\hline 53 & 225 & 0.56892 & 0.063615 & 0.91175 & 0.34862 \\
\hline 54 & 235 & 0.54051 & 0.058165 & 0.76062 & 0.3962 \\
\hline 55 & 245 & 0.5264 & 0.058327 & 0.72832 & 0.41611 \\
\hline 56 & 255 & 0.52244 & 0.054895 & 0.93194 & 0.44834 \\
\hline 57 & 265 & 0.5238 & 0.05449 & 0.72243 & 0.3846 \\
\hline 58 & 275 & 0.51284 & 0.05305 & 0.58923 & 0.34188 \\
\hline 59 & 285 & 0.52235 & 0.054618 & 0.55248 & 0.28383 \\
\hline 60 & 295 & 0.52513 & 0.053787 & 0.47665 & 0.28428 \\
\hline 61 & 305 & 0.54252 & 0.059891 & 0.39245 & 0.30651 \\
\hline 62 & 315 & 0.52833 & 0.058073 & 0.32065 & 0.26403 \\
\hline 63 & 325 & 0.52646 & 0.060293 & 0.26349 & 0.26242 \\
\hline 64 & 335 & 0.51418 & 0.057107 & 0.19495 & 0.31293 \\
\hline 65 & 345 & 0.5019 & 0.059131 & 0.19367 & 0.31006 \\
\hline 66 & 355 & 0.4817 & 0.056403 & -0.13986 & 0.25456 \\
\hline 67 & 365 & 0.49184 & 0.05857 & -0.26604 & 0.23646 \\
\hline 68 & 375 & 0.48162 & 0.05596 & -0.13604 & 0.25717 \\
\hline 69 & 385 & 0.48441 & 0.058584 & 0.118 & 0.21237 \\
\hline 70 & 395 & 0.48331 & 0.055877 & 0.11072 & 0.18126 \\
\hline 71 & 405 & 0.47328 & 0.052872 & 0.2847 & 0.22181 \\
\hline 72 & 415 & 0.46114 & 0.053132 & 0.49842 & 0.23663 \\
\hline 73 & 425 & 0.46716 & 0.054852 & 0.46395 & 0.29863 \\
\hline 74 & 435 & 0.4644 & 0.053609 & 0.37135 & 0.27079 \\
\hline 75 & 445 & 0.45077 & 0.053822 & 0.17287 & 0.22165 \\
\hline 76 & 455 & 0.4492 & 0.055065 & 0.26648 & 0.20773 \\
\hline 77 & 465 & 0.44914 & 0.05482 & 0.15332 & 0.24302 \\
\hline 78 & 475 & 0.43461 & 0.053617 & 0.070683 & 0.25635 \\
\hline 79 & 485 & 0.43768 & 0.056573 & 0.063862 & 0.23943 \\
\hline 80 & 495 & 0.44366 & 0.053398 & 0.24806 & 0.27574 \\
\hline
\end{tabular}

S7 Data. The data presented in S2 Fig.

Note:

FigS2PSTH monkeyH.txt contains the values for the population average PSTHs for monkey $\mathrm{H}$ in Figure S2

FigS2PSTH_monkeyE.txt contains the values for the population average PSTHs for monkey E in Figure S2 
FigS2PSTH_monkeyH:

Time_(ms) Mean_attentional_effect $\mathrm{Cl}$

$1-295$

$2-285$

$3-275$

$4-265$

$5-255$

$6-245$

$7 \quad-235$

$8-225$

$9-215$

$10-205$

$11-195$

$12-185$

$13-175$

$14-165$

$15-155$

$16-145$

$17-135$

$18-125$

$19-115$

$20-105$

$21-95$

$22-85$

$\begin{array}{ll}23 & -75\end{array}$

$24-65$

$25-55$

$26-45$

$\begin{array}{ll}27 & -35\end{array}$

$28-25$

$29-15$

$30-5$

315

3215

3325

$34 \quad 35$

$35 \quad 45$

$36 \quad 55$

$37 \quad 65$

3875

3985

$40 \quad 95$

$41 \quad 105$

$42 \quad 115$

$43 \quad 125$

$44 \quad 135$

$45 \quad 145$

$46 \quad 155$

$47 \quad 165$

$48 \quad 175$

49185

$50 \quad 195$

51205

$52 \quad 215$

$53 \quad 225$

$54 \quad 235$

$55 \quad 245$

$56 \quad 255$

$57 \quad 265$

$58 \quad 275$

59285

\begin{tabular}{lc|ll}
0.58137 & 2.0834 & 64 & 335 \\
-0.2098 & 1.7531 & 65 & 345 \\
0.18278 & 1.7038 & 66 & 355 \\
-0.29116 & 1.672 & 67 & 365 \\
-0.45375 & 1.7414 & 68 & 375 \\
0.6861 & 1.9764 & 69 & 385 \\
1.159 & 1.8704 & 70 & 395 \\
-0.095437 & 1.8282 & 71 & 405 \\
-0.48814 & 1.9327 & 72 & 415 \\
-0.56252 & 1.7543 & 73 & 425 \\
-0.82488 & 1.9822 & 74 & 435 \\
-0.43742 & 2.0904 & 75 & 445 \\
0.16235 & 2.2372 & 76 & 455 \\
1.323 & 2.2254 & 77 & 465 \\
0.85268 & 2.6838 & 78 & 475 \\
1.3076 & 3.1539 & 79 & 485 \\
0.83204 & 2.7651 & 80 & 495
\end{tabular}

$\begin{array}{lc}2.0902 & 3.8349 \\ 1.7857 & 3.7985 \\ 2.8525 & 3.7617 \\ 3.614 & 3.8046 \\ 5.1329 & 3.918 \\ 6.9315 & 4.021 \\ 8.0287 & 4.0042 \\ 9.5929 & 4.0122 \\ 10.3884 & 4.6736 \\ 11.5895 & 4.4531 \\ 11.9875 & 4.4723 \\ 12.4644 & 4.1003 \\ 12.8581 & 4.3197 \\ 11.7018 & 4.399 \\ 11.0352 & 4.5748 \\ 10.9372 & 4.8373 \\ 10.3114 & 4.895 \\ 9.8787 & 4.4818 \\ 9.1411 & 4.4496 \\ 8.667 & 4.2825 \\ 8.2812 & 4.3975\end{array}$

FigS2PSTH_monkeyE:

\begin{tabular}{|c|c|c|c|}
\hline & Time (ms) & \multicolumn{2}{|c|}{ Mean attentional effect } \\
\hline 1 & -295 & $0.8736 \overline{7}$ & 1.4899 \\
\hline 2 & -285 & 0.879 & 1.4558 \\
\hline 3 & -275 & 0.26427 & 1.7511 \\
\hline 4 & -265 & 0.24599 & 1.7091 \\
\hline 5 & -255 & 0.039469 & 1.6269 \\
\hline 6 & -245 & -0.47551 & 1.7079 \\
\hline 7 & -235 & -0.82249 & 1.6776 \\
\hline 8 & -225 & -0.83786 & 1.5989 \\
\hline 9 & -215 & -1.0577 & 1.8025 \\
\hline 10 & -205 & -0.9445 & 1.6527 \\
\hline 11 & -195 & -0.69962 & 1.438 \\
\hline 12 & -185 & 0.10182 & 1.3538 \\
\hline 13 & -175 & 0.23558 & 1.5079 \\
\hline 14 & -165 & 0.21188 & 1.3539 \\
\hline 15 & -155 & -0.58115 & 1.2568 \\
\hline 16 & -145 & 0.63103 & 1.5457 \\
\hline 17 & -135 & 0.35163 & 1.5634 \\
\hline 18 & -125 & 0.27468 & 1.5281 \\
\hline 19 & -115 & 0.25633 & 1.6703 \\
\hline 20 & -105 & 0.94704 & 1.889 \\
\hline 21 & -95 & -0.26838 & 1.8503 \\
\hline 22 & -85 & -0.3316 & 1.7863 \\
\hline 23 & -75 & -0.73648 & 1.9131 \\
\hline 24 & -65 & -0.68576 & 1.3362 \\
\hline 25 & -55 & -1.2533 & 1.7189 \\
\hline 26 & -45 & -0.51178 & 1.7934 \\
\hline 27 & -35 & -0.58727 & 1.657 \\
\hline 28 & -25 & -0.065759 & 1.9832 \\
\hline 29 & -15 & 0.47966 & 2.2304 \\
\hline 30 & -5 & 1.3192 & 2.0301 \\
\hline 31 & 5 & 1.139 & 2.68 \\
\hline 32 & 15 & -0.26131 & 3.0205 \\
\hline 33 & 25 & -0.32825 & 3.2324 \\
\hline 34 & 35 & -2.834 & 3.5804 \\
\hline 35 & 45 & -2.156 & 3.6898 \\
\hline 36 & 55 & -1.935 & 3.9241 \\
\hline 37 & 65 & -0.66879 & 3.6644 \\
\hline
\end{tabular}




$\begin{array}{llll}38 & 75 & 1.2557 & 3.2863 \\ 39 & 85 & 3.8835 & 3.5045 \\ 40 & 95 & 3.9376 & 3.489 \\ 41 & 105 & 5.6002 & 2.8703 \\ 42 & 115 & 7.9642 & 2.473 \\ 43 & 125 & 8.1909 & 3.1376 \\ 44 & 135 & 9.3435 & 3.4418 \\ 45 & 145 & 10.3776 & 4.1636 \\ 46 & 155 & 7.9946 & 3.6993 \\ 47 & 165 & 5.8416 & 3.9476 \\ 48 & 175 & 6.4685 & 3.6792 \\ 49 & 185 & 5.2322 & 3.6579 \\ 50 & 195 & 3.5225 & 3.3327 \\ 51 & 205 & 5.6484 & 3.6555 \\ 52 & 215 & 8.4299 & 3.8193 \\ 53 & 225 & 6.7573 & 4.0693 \\ 54 & 235 & 8.6368 & 3.931 \\ 55 & 245 & 9.1342 & 3.9831 \\ 56 & 255 & 7.3508 & 4.4167 \\ 57 & 265 & 6.8344 & 4.8109 \\ 58 & 275 & 6.9894 & 5.5497 \\ 59 & 285 & 4.8494 & 5.1383 \\ 60 & 295 & 4.3564 & 5.1865 \\ 61 & 305 & 5.6535 & 4.8102 \\ 62 & 315 & 4.6821 & 3.9457 \\ 63 & 325 & 4.2472 & 4.2154 \\ 64 & 335 & 5.5825 & 3.9402 \\ 65 & 345 & 6.6659 & 3.6192 \\ 66 & 355 & 7.3216 & 4.0849 \\ 67 & 365 & 7.0167 & 4.2446 \\ 68 & 375 & 8.2359 & 4.3935 \\ 69 & 385 & 8.6967 & 3.7828 \\ 70 & 395 & 7.5067 & 3.563 \\ 71 & 405 & 6.3975 & 3.3548 \\ 72 & 415 & 7.6455 & 3.4001 \\ 73 & 425 & 7.0548 & 3.8473 \\ 74 & 435 & 5.8643 & 4.2205 \\ 75 & 445 & 7.784 & 4.4458 \\ 76 & 455 & 8.6023 & 4.5583 \\ 77 & 465 & 6.9934 & 4.7635 \\ 78 & 475 & 6.5831 & 4.2317 \\ 79 & 485 & 6.8496 & 3.9354 \\ 80 & 495 & 3.3792 & 3.5464 \\ & & & \end{array}$


Additional supplementary materials 4: Example epars \& mpars

Note: The epars define the time sequence of the experiment, the location of the stimulus, i.e. when and where to show the stimulus on the screen. The epars also on-line control the data collection and some events related to subject performance (such as eye position, time window of reaction etc.). The mpars controls the properties of the stimuli, such as the motion direction, speed, dot density, color of the RDP.

\section{Example epars:}

projectLeader tao

expCode trAttC

displayWindowOnSecondMonitor 00200200

trialProtocol 19

0000010

$\begin{array}{llllll}55 & 0 & -320 & -380 & 1 & 1\end{array}$

$\begin{array}{llllll}10 & 20 & -320 & -380 & 1 & 1\end{array}$

1000000

$\begin{array}{llllll}55 & 0 & -320 & 380 & 1 & 1\end{array}$

$\begin{array}{llllll}10 & 20 & -320 & 380 & 1 & 1\end{array}$

10500011

$\begin{array}{llllll}55 & 59 & -320 & -380 & 1 & 1\end{array}$

$\begin{array}{lllllll}55 & 59 & -320 & 380 & 1 & 1\end{array}$

$\begin{array}{llll}\text { displayCenter } 0 & 1 & 0.29167 & 0.5\end{array}$

displayCenter $1 \quad 1800 \quad 0$

filenames\&pathname 35 :trace:traceMpars:

Luminance.mpar

fixStim1.mpar

fixStim2.mpar

Cue.mpar

TP.mpar

T45.mpar

T90.mpar

T135.mpar

T180.mpar

T225.mpar

T270.mpar

T315.mpar

DP.mpar

D45.mpar

D90.mpar

D135.mpar

D180.mpar

D225.mpar

D270.mpar
D315.mpar

AttTP.mpar

AttDP.mpar

DPshort.mpar

fixStimlong.mpar

DPOff.mpar

AttTPF.mpar

AttDPF.mpar

D1800ff.mpar

fixStim4.mpar

fixStim5.mpar

fixStim6.mpar

AttTP180.mpar

AttDP180.mpar

AttTPF180.mpar

AttDPF180.mpar

\# class 5 and 6 fixation task ( +60 frame to

+120 frame, $+\sim 800 \mathrm{~ms}$ to $1600 \mathrm{~ms}$ )

\# class 1 and 2 phase change after 1 st FP

disappears $(+20$ to +100 frames, $\sim+267 \mathrm{~ms}$ to

$+1333 \mathrm{~ms}$ after 1st FP disappears)

\# class 3 and 4 are prefered directions, cue in cue out

\# class 7 and 8 are antiprefered directions,

cue in cue out

\# class 9-12 antipreference directions,

before(11-12) and after saccade(9-10).

classes 19

1000100000000

12921402203100100

12922002143100200

120400032525300

120000432525400

1302640270000500

1302700264000600

120400032828700

120000432828800

12932403303100900

129330032431001000

13034403500001100

13035003440001200

1204000325251300

1200004325251400

1204000328281500

1200004328281600

12000003001700

12000003001800

classFrequencyOption 0 
\# default is zero, this equates the number of trials

waitForLever 10000

rewardDuration 90

leverDownRewardDuration 0

preLeverBeepDelay 2000

anticipatedResponseDuration 150

responseTimeWindow 600

\#changed above from 500

hitDelay 1500

missDelay 1000

leverReleaseDuration 600

juicePin 1

fixpointIndexColor 255

backgroundIndexColor 200

fixPointSize 12

keyboardResponse FALSE

earlyResponsePermitted FALSE

fixPointVisibleBetweenTrials false

fixationRequired FALSE

collectEyePositions TRUE

fixAreaRadiusOfClass 080

preLeverFixRelaxation 2

smoothEyePositions 4

spikeSource 1

sampleSource 1

collectSpikes TRUE

numberOfTrials 9000

dotPosToBuffer FALSE

spikeRecSystem 3

eyePosDecay -20

provideWords TRUE

openDataFile

savePupilDiameter TRUE

\section{Example mpars}

fixStim1.mpar:

numberOfSurfaces 1400

pixelradius 20

numberOfFrames 105

\#qtRGBForeColor

655365530

qtIndexForeColor 255

$\begin{array}{llllll}\text { qtSquare } & -6 & -6 & 6 & 6\end{array}$

\section{fixStim2.mpar}

numberOfSurfaces 1400

pixelradius 10

numberOfFrames 105

\#qtRGBForeColor

qtIndexForeColor 255

$\begin{array}{lllll}\text { qtSquare } & -6 & -6 & 6 & 6\end{array}$

qtSuspendFix 105125

qtFixFactor $\quad 105-1 \quad 1.5$

qtFixDispCtr $\quad 105-1 \quad 1$

\author{
fixStim6.mpar \\ numberOfSurfaces 1400 \\ pixelradius 10 \\ numberOfFrames 300 \\ \#qtRGBForeColor $\quad 655365530$ \\ qtIndexForeColor 200 \\ $\begin{array}{lllll}\text { qtSquare } & -6 & -6 & 6 & 6\end{array}$ \\ qtSuspendFix 105125 \\ qtFixFactor $\quad 105-1 \quad 1.5$ \\ qtFixDispCtr $105-1 \quad 1$ \\ DPOff.mpar \\ numberOfSurfaces 14010 \\ dotSize 4 \\ pixelradius 100 \\ numberOfFrames 59 \\ speed 8 \\ shape circle \\ colorIndex 255 \\ direction 240

\section{AttTPF.mpar} \\ numberOfSurfaces 14010 \\ dotSize 4 \\ pixelradius 100 \\ numberOfFrames 150 \\ speed 8 \\ shape circle \\ colorIndex 255 \\ direction 240 \\ changePhase -60 140 \\ directionTable 2150330 \\ changePhase 1010 \\ direction 240
}

\section{AttTP.mpar}

numberOfSurfaces 14010

dotSize 4

pixelradius 100

numberOfFrames 250

speed 8

shape circle

colorIndex 255

direction 240

changePhase -125 205

directionTable 2150330

changePhase 1010

direction 240

\section{AttDP.mpar}

numberOfSurfaces 14010

dotSize 4

pixelradius 100

numberOfFrames 250 
speed 8

shape circle

colorIndex 255

direction 240

changePhase 125205

directionTable 2150330

changePhase 1010

direction 240

\section{Cue.mpar}

numberOfSurfaces

$1 \quad 40 \quad 10$

dotSize 4

pixelradius 80

numberOfFrames

speed 0

shape circle

colorIndex

\section{D1800ff.mpar}

numberOfSurfaces 14010

dotSize 4

pixelradius 100

numberOfFrames 59

speed 8

shape circle

colorIndex 255

direction 60

\section{AttDP180.mpar}

numberOfSurfaces 14010

dotSize 4

pixelradius 100

numberOfFrames 250

speed 8

shape circle

colorIndex 255

direction 60

changePhase 125205

directionTable 2330150

changePhase 1010

direction 60 
Chapter 5

General discussion 
Humans and monkeys make two to three saccades per second on average when they are awake (Snodderly, 1987), and they are able to keep track of relevant visual stimuli while making saccadic eye-movements to scan a visual scene. Since the visual system mostly operates using retinotopic representations (Wurtz, 2008; Cavanagh, Hunt, Afraz et al., 2010; Marino and Mazer, 2016), in each visual area, a relevant visual stimulus (the target) at a fixed spatial location is represented by one neuronal population before the saccade and a different neuronal population after the saccade: we refer to these as the pre-saccadic target population and the post-saccadic target population respectively. As a result, to maximally and selectively enhance target processing (but not distractor processing) both before and after the saccade, a rapid, saccade-synchronized remapping of top-down attentional modulation from the pre-saccadic to the post-saccadic target population is optimal. Attentional enhancement would ideally be expected to be dominant at the presaccadic target population until just before saccade offset, and decay at or soon after saccade offset. Similarly, attentional enhancement would be expected to emerge at the post-saccadic target population at or soon after saccade offset. However, the time-course of the shift of top-down attention from the pre-saccadic to the post-saccadic target population across a saccade has never been explicitly measured.

We recorded the extracellular activity of single neurons in area MT of two macaque monkeys during a task that required them to make a saccade while maintaining attention on one of four moving random dot patterns (RDPs). In the first experiment, the attended stimulus was either in the neurons' pre-saccadic receptive field (RF) or directly opposite to it, while in the second experiment, the attended stimulus was either in the post-saccadic RF or directly opposite to it. By looking at the neuronal responses just before and after the eye movement, we were able to investigate the time course of the neuronal activity when the target was brought into or moved out of the neuron's RF by the saccade. Therefore, we investigated how attention modulation emerged and decayed in the neuronal activity in area MT across saccades by this experimental manipulation. We found, for the first time, that trans-saccadic attentional shift is well-synchronized to the saccade: attentional enhancement crosses over from the pre-saccadic to the post-saccadic target population at 31 and 52 ms after saccade offset in the two monkeys in area MT. Specifically, the results of our first experiment indicated that attentional enhancement of the pre-saccadic target population lingered after the saccade and disappeared by $50 \mathrm{~ms}$ after saccade offset in one monkey and by $100 \mathrm{~ms}$ in the other; and the results of our second experiment suggest 
attentional enhancement of the post-saccadic target population emerged at saccade offset and within $50 \mathrm{~ms}$ of saccade offset in the two monkeys. We did not find any evidence for attentional enhancement of the post-saccadic target population before the saccade, even though our experimental design ensured that there would be a distractor-driven response before the saccade on which an attentional effect could be seen, if present.

By using a similar paradigm with finer temporal resolution, we also showed that the topdown spatial attention is fully available at the task-relevant location within 30 milliseconds after the saccade in human subjects. Given an onset latency of approximately $30 \mathrm{~ms}$ in MT (Bair, Cavanaugh, Smith et al., 2002), a visual change occurring $30 \mathrm{~ms}$ after saccade offset would reach MT by $60 \mathrm{~ms}$ after saccade offset, by which time attention would have crossed-over to the post-saccadic target population (as we show here). Further, our data indicated that under our task conditions, subjects very rarely confused a distractor stimulus for the target. Spatial attention and saccadic execution thus appeared to coordinate well to ensure that relevant objects were attentionally enhanced soon after the beginning of each eye fixation. Additionally, we did not find any evidence for a predictive, pre-saccadic shift of attention to the post-saccadic target population when the human subjects were doing the task. This is consistent with the fact that we and others (cite Ong and Bisley) have not found evidence for "predictive" pre-saccadic attentional remapping to the post-saccadic target population. The absence of predictive remapping in MT is particularly interesting, because a large body of previous work indicates that neurons in the lateral intraparietal area (LIP), frontal eye field (FEF), superior colliculus (SC), medial superior temporal area (MST) and in the ventral stream (areas V3a, V3 and V2) respond peri-saccadically as long as a visual stimulus could be anticipated in their receptive fields (RFs) after the saccade (Duhamel, Colby and Goldberg, 1992; Walker, Fitzgibbon and Goldberg, 1995; Umeno and Goldberg, 1997, 2001; Nakamura and Colby, 2002; Wurtz, Joiner and Berman, 2011; Inaba and Kawano, 2014). This "remapped response" is not a simple visual afferent response, because it appears even when the visual stimulus disappears just before the saccade (that would bring the stimulus location into the RF), so that no stimulus ever appears in the neurons' visual RF before or after the saccade. Further, in some neurons, it begins with a latency shorter than the normal visual latency and can even begin before saccade onset, in which case it has been referred to as "predictive remapping" (Duhamel, Colby and Goldberg, 1992). More commonly, the remapped response occurs post-saccadically, and when this occurs in a situation where there is no 
post-saccadic stimulus in the RF because it disappeared before the saccade, the remapped response is referred to as a "memory trace" of the location of the visual stimulus (Duhamel, Colby and Goldberg, 1992). Though this anticipatory activity has not been studied explicitly in conditions evoking top-down spatial attention, predictive activity is greater for stimuli with greater bottom-up saliency (Gottlieb, Kusunoki and Goldberg, 1998; Joiner, Cavanaugh and Wurtz, 2011) and for stimuli that are learnt visual search targets (Phillips and Segraves, 2010; Mirpour and Bisley, 2012) or saccade targets (Gottlieb, Kusunoki and Goldberg, 1998). We hypothesize that the anticipatory remapping seen in attentional and oculomotor control areas like LIP, FEF and SC is part of the process that enables attentional remapping in MT that is well synchronized to the saccade. In other words, even though this process starts before the saccade in these areas, its effects in MT, with which these areas are strongly connected (Maunsell and van Essen, 1983; Ungerleider and Desimone, 1986; Blatt, Andersen and Stoner, 1990), only manifest after the saccade. In this view, the previous results on trans-saccadic remapping represent the predictive, pre-saccadic shift of attentional pointers on a retinotopic map that keeps track of attended locations across saccades (Cavanagh, Hunt, Afraz et al., 2010), so that attended locations can be preferentially processed with minimal delay after the saccade (Yao, Treue and Krishna, 2016). This reduction of delay would be especially helpful when planning rapid sequential saccades and could also help maintain an uninterrupted visual experience across saccades.

Though we did not find predictive remapping in MT, we did find evidence for a postsaccadic remapped response (i.e. a memory trace) in MT. To do this, we designed a new remapping paradigm that was different from the traditional one and recorded neuronal activity. The monkeys in this study were required to perform a task very similar to the first study, i.e. making a saccade while the fixation point jumped and responding to the motion change of a target stimulus (moving in the preferred or anti-preferred direction of the recorded neuron) while ignorinig distractors. The target stimulus could be in the RF or out of it in different conditions. In a very important condition, the stimuli were removed just before the saccade onset so that it never appeared in the neuron's RF. The responses in this condition enabled us to look for the presence of a remapped response, and ask if the remapped response was modulated by top-down attention and whether it contained information about visual features (motion direction in our study). We found evidence for a memory trace, in MT. The memory trace was modulated by top-down spatial attention: i.e. the neuronal response after the saccade signaled whether a target or a distractor stimulus 
was present in its RF before the saccade. This was the first demonstration, to our knowledge, of the top-down attention influence on remapping activity. We also investigated whether the memory trace contained information about motion direction. We found only a small and transient effect of motion direction on the memory trace in only one monkey, suggesting that the remapped response did not signal the motion direction of the stimulus in the RF before the saccade. These data on the memory trace in MT contribute to an ongoing debate regarding the functional role of trans-saccadic remapping. The presence of feature-related information in the remapped response has become a critical test that distinguishes between two alternative views of the functional role of trans-saccadic remapping that are being actively debated (Wurtz, 2008; Cavanagh, Hunt, Afraz et al., 2010; Mayo and Sommer, 2010; Melcher, 2010; Zirnsak and Moore, 2014). Absence of featural information in the remapped response would support the proposal (Cavanagh, Hunt, Afraz et al., 2010) that trans-saccadic remapping represents the predictive, pre-saccadic shift of attentional pointers on a retinotopic map that keeps track of attended locations across saccades. On the other hand, the presence of featural information in the remapped response would support the proposal that trans-saccadic remapping plays an additional role in spatiotopic feature comparison and adaptation transfer across saccades (Melcher and Colby, 2008; Mayo and Sommer, 2010; Melcher, 2010; Crapse and Sommer, 2012), Our data from MT clearly support the former proposal. We propose that the memory trace emerges from the rapid shift of attention to the post-saccadic neuronal population and the resultant enhancement of the baseline firing-rate. The absence of motion-direction information in the memory trace is consistent with a shift of a purely spatial, top-down attentional signal. However, more data from different visual areas (including those in the ventral stream) are needed to fully decide this debate.

In summary, the physiological data presented here, combined with our human psychophysics results support our hypothesis that spatial attention and saccadic processing co-ordinate well to ensure that relevant locations are attentionally enhanced soon after the beginning of each eye fixation, and can be tracked and rapidly processed across saccades. 


\section{References:}

Bair W, Cavanaugh JR, Smith MA, Movshon JA (2002) The timing of response onset and offset in macaque visual neurons. J Neurosci 22:3189-3205.

Blatt GJ, Andersen RA, Stoner GR (1990) Visual receptive field organization and cortico-cortical connections of the lateral intraparietal area (area LIP) in the macaque. J Comp Neurol 299:421-445.

Cavanagh P, Hunt AR, Afraz A, Rolfs M (2010) Visual stability based on remapping of attention pointers. Trends Cogn Sci 14:147-153.

Crapse TB, Sommer MA (2012) Frontal eye field neurons assess visual stability across saccades. J Neurosci 32:2835-2845.

Duhamel JR, Colby CL, Goldberg ME (1992) The updating of the representation of visual space in parietal cortex by intended eye movements. Science 255:90-92.

Gottlieb JP, Kusunoki M, Goldberg ME (1998) The representation of visual salience in monkey parietal cortex. Nature 391:481-484.

Inaba N, Kawano K (2014) Neurons in cortical area MST remap the memory trace of visual motion across saccadic eye movements. Proc Natl Acad Sci U S A 111:7825-7830.

Joiner WM, Cavanaugh J, Wurtz RH (2011) Modulation of shifting receptive field activity in frontal eye field by visual salience. J Neurophysiol 106:1179-1190.

Marino AC, Mazer JA (2016) Perisaccadic Updating of Visual Representations and Attentional States: Linking Behavior and Neurophysiology. Front Syst Neurosci 10:3.

Maunsell JH, van Essen DC (1983) The connections of the middle temporal visual area (MT) and their relationship to a cortical hierarchy in the macaque monkey. J Neurosci 3:2563-2586.

Mayo JP, Sommer MA (2010) Shifting attention to neurons. Trends Cogn Sci 14:389; author reply 390-381.

Melcher D (2010) The missing link for attention pointers: comment on Cavanagh et al. Trends Cogn Sci 14:473; author reply 474-475.

Melcher D, Colby CL (2008) Trans-saccadic perception. Trends Cogn Sci 12:466-473.

Mirpour K, Bisley JW (2012) Anticipatory remapping of attentional priority across the entire visual field. J Neurosci 32:16449-16457.

Nakamura K, Colby CL (2002) Updating of the visual representation in monkey striate and extrastriate cortex during saccades. Proc Natl Acad Sci U S A 99:4026-4031.

Phillips AN, Segraves MA (2010) Predictive activity in macaque frontal eye field neurons during natural scene searching. J Neurophysiol 103:1238-1252.

Snodderly DM (1987) Effects of light and dark environments on macaque and human fixational eye movements. Vision Res 27:401-415.

Umeno MM, Goldberg ME (1997) Spatial processing in the monkey frontal eye field. I. Predictive visual responses. J Neurophysiol 78:1373-1383.

Umeno MM, Goldberg ME (2001) Spatial processing in the monkey frontal eye field. II. Memory responses. J Neurophysiol 86:2344-2352.

Ungerleider LG, Desimone R (1986) Cortical connections of visual area MT in the macaque. J Comp Neurol 248:190-222.

Walker MF, Fitzgibbon EJ, Goldberg ME (1995) Neurons in the monkey superior colliculus predict the visual result of impending saccadic eye movements. J Neurophysiol 73:1988-2003.

Wurtz RH (2008) Neuronal mechanisms of visual stability. Vision Res 48:2070-2089.

Wurtz RH, Joiner WM, Berman RA (2011) Neuronal mechanisms for visual stability: progress and problems. Philos Trans R Soc Lond B Biol Sci 366:492-503.

Yao T, Treue S, Krishna BS (2016) An Attention-Sensitive Memory Trace in Macaque MT Following Saccadic Eye Movements. PLoS Biol 14:e1002390.

Zirnsak M, Moore T (2014) Saccades and shifting receptive fields: anticipating consequences or selecting targets? Trends Cogn Sci 18:621-628. 


\section{Acknowledgements}

I would like to appreciate everybody who helped me and accompanied me in last years.

At first, I would like to express my deepest gratitude to my supervisor, Prof. Stefan Treue, who picked me up after I got my master degree at which time I spoke very pool English, and gave me a chance to pursue a PhD degree in Germany. He leads a fantastic lab with a friendly and comfortable scientific and life atmosphere. He always gives us critical ideas about our projects, which results to better experiment design and improved results.

Secondly, I would like to thank Dr. Suresh Krishna, my co-supervisor and the closest working partner in last six years. He helped in every aspect of this dissertation. I can hardly express my gratitude to him for his help. This thesis would not have reached its current stage without his constructive instruction and help. I would especially thank him for his patience when we are discussing.

Thirdly, I would like to thank Prof. Alexander Gail, one of my thesis committee members, and Dr. Igor Kagan for their discussion and suggestions on my projects. I also appreciate Dr. Cliodhna Quigley's proofreading on the general introduction of this thesis.

I should also appreciate our fantastic technician team: Ralf Brockhausen is always helpful on the software and hardware of the setups and computers; Beatrix Glaser helped my daily administrative affairs; Leonore Burchardt, Dirk Prüsse, Sina Plümer helped me on animal training and caring; Klaus Heisig, as one of my ex-officemate, helped me deal with engineer issues of the setup. 
I thank all of the lab members in CNL. I had very good time in Germany because of you. I am glad to have Xue Cheng, Guo Hao, Kuang Shenbing, and Liu Ruifeng in the lab; we had good Chinese time.

At Last but not least, I would like to thank my families. My parents have always been supporting me to do anything I want to do. My sister took a lot of family responsibilities when I was not in China.

Thank YOU for your Large Odd Vegas Eagle!

Thank the sun shining on earth!!

感谢阳光普照大地！！！！ 
